\title{
Theory of weights in $p$-adic cohomology
}

\author{
Tomoyuki Abe and Daniel Caro
}

\begin{abstract}
Let $k$ be a finite field of characteristic $p>0$. We construct a theory of weights for overholonomic complexes of arithmetic $\mathcal{D}$-modules with Frobenius structure on varieties over $k$. The notion of weight behave like Deligne's one in the $\ell$-adic framework: first, the six operations preserve weights, and secondly, the intermediate extension of an immersion preserves pure complexes and weights.
\end{abstract}

\section{Contents}

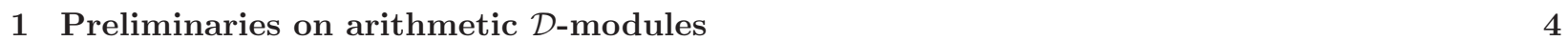

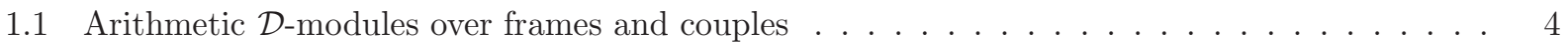

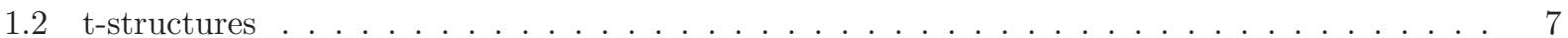

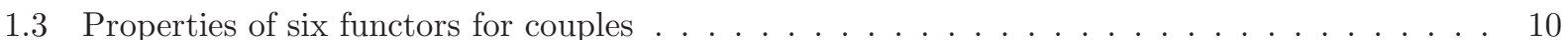

1.4 Intermediate extensions $\ldots \ldots \ldots \ldots \ldots \ldots \ldots \ldots$

1.5 Local theory on formal disk . . . . . . . . . . . . . . . . . . . . . . . 19

2 Mixed complexes $\quad 23$

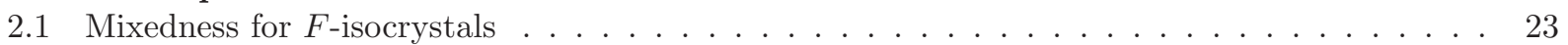

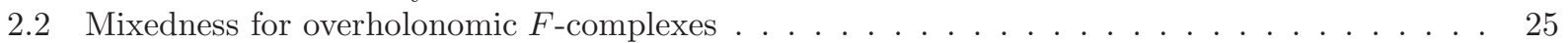

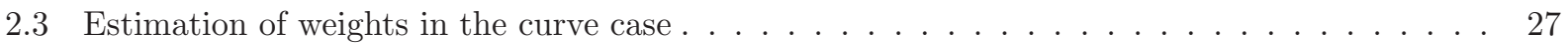

3 Monodromy filtration of a convergent log-isocrystal $\quad 29$

3.1 Notation . . . . . . . . . . . . . . . . . . . . . . . 30

3.2 Monodromy filtration of a convergent log-isocrystal with nilpotent residues . . . . . . . . 32

3.3 Comparison with Crew's Frobenius structure . . . . . . . . . . . . . . . . . 35

3.4 Relations between a convergent log-isocrystal and its associated overconvergent isocrystal . . 37

3.5 An isomorphism compatible with Frobenius . . . . . . . . . . . . . . . . . . 45

3.6 Stability of mixedness for a unipotent $F$-isocrystal $\ldots \ldots \ldots \ldots \ldots \ldots$

4 Theory of weights $\quad \mathbf{5 4}$

4.1 Stability of mixedness $\ldots \ldots \ldots \ldots \ldots \ldots \ldots \ldots \ldots \ldots \ldots$

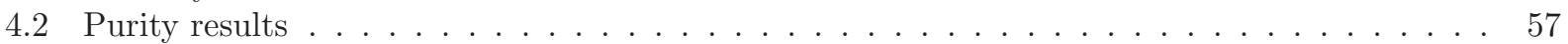

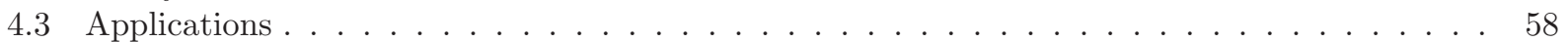

$\begin{array}{ll}\text { A Internal homomorphism } & 61\end{array}$

\section{Introduction}

Using Grothendieck's theory of $\ell$-adic étale cohomology of varieties over a field of characteristic $p \neq \ell$, Deligne proved Weil's conjectures on the numbers of points of algebraic varieties over finite fields. Moreover, he built a theory of mixedness and weights for constructible $\ell$-adic sheaves which is compatible with the "six operations formalism" of the $\ell$-adic cohomology, namely the mixedness and weight are stable under the operations $f_{!}, f_{*}, f^{*}, f^{!}, \otimes$ and $\mathcal{H}$ om (see [Del80]). Later, using the theory of perverse sheaves, in 
[BBD82] Gabber proved the stability of purity and mixedness under intermediate extensions, with which the theory of weights for $\ell$-adic cohomology may be regarded as complete. However, the problem of obtaining similar results within a $p$-adic cohomological framework remained opened. After Dwork's $p$-adic proof of the rationality of zeta functions, a part of Weil's conjectures, it seems natural to expect better computability of zeta functions with a $p$-adic approach. In this paper, we build a $p$-adic theory of weights.

The first attempt to calculate the weights of some $p$-adic cohomology was made by Katz and Messing in their famous paper [KM74]. Using Deligne's deep results on weights, especially "Le théorème du pgcd", they showed that, for projective smooth varieties, the weight of crystalline cohomology is the same as that of $\ell$-adic one. It is reasonable to hope that the coefficient theory of weights parallel to $\ell$-adic cohomology should exist in the spirit of the petit camarade conjecture [Del80, 1.2.10], even though there were many obstacles that prevented the construction of such a theory. After the work of Katz-Messing, efforts were made until Kedlaya finally obtained in [Ked06] the expected estimation of weights of rigid cohomology, a $p$-adic cohomology constructed by Berthelot. We do not go into more details of the history, and recommend the reader to consult the excellent explanation in the introduction of [Ked06].

But the context of rigid cohomology was still not completely satisfactory, in the sense that this is not "functorial enough", namely we do not have six operations formalism, specially push-forwards as pointed out in $[$ Ked06, 5.3.3]. In this paper, we use systematically Berthelot's arithmetic $\mathcal{D}$-modules to complete the program of establishing a $p$-adic theory of weights stable under six operations. In many applications, such a theory should play as important roles as suggested by the classical situations; for example, the theory of intersection cohomology and its purity, the theory of Springer representations, Lafforgue's proof of Langlands correspondence, etc. In the final part of the paper, we show one such application that the Hasse-Weil $L$-function for a function field defined by means of $\ell$-adic methods and $p$-adic methods coincide.

Now let us explain our results in more details. Let $\mathcal{V}$ be a complete discretely valued ring of mixed characteristic $(0, p), K$ its field of fractions, and $k$ its residue field which is assumed to be a finite field. We suppose that there exists a lifting of the Frobenius of $k$ to $\mathcal{V}$. In order to obtain a $p$-adic cohomology on algebraic varieties over $k$ stable under the six operations whose coefficients contain the category of overconvergent isocrystals, Berthelot constructed the theory of arithmetic $\mathcal{D}$-modules (cf. [Ber02]). Arithmetic $\mathcal{D}$-modules may be seen as a $p$-adic analogue of modules over the ring of differential operators over complex varieties or analytic varieties. Berthelot's theory is inspired by his construction of rigid or crystalline cohomology, and we consider not only differential operators of finite order, but also of infinite order subject to a convergence condition. Even though the objects are defined, the preservation of finiteness had been a difficult question. For this, the notion of overholonomicity was introduced by the second author in [Car09b]. Thanks to Kedlaya's semistable reduction theorem of [Ked11], the second author together with Tsuzuki, finally in [CT12], proved that overholonomicity of $\mathcal{D}$-modules with Frobenius structure is preserved under various cohomological functors.

Using this foundation, in this paper, we go a step further in the development of a good $p$-adic cohomology, and build a theory of weights for overholonomic complexes with Frobenius structure of arithmetic $\mathcal{D}$-modules. More precisely, the main result is the following (cf. Theorem 4.1.3, Corollary 4.2.4):

Main Theorem. Let $X$ be a (realizable) variety over $k$. We construct the full subcategory $F-D_{\mathrm{m}}^{\mathrm{b}}(X / K)$ of $F-D_{\text {ovhol }}^{\mathrm{b}}(X / K)$ of $\iota$-mixed complexes. The following properties hold:

(i) Let $f: X \rightarrow Y$ be a morphism of varieties. The $\iota$-mixedness is stable under functors $f_{+}, f_{!}, f^{+}$, $f^{!}, \mathbb{D}_{X}, \widetilde{\otimes}$. More precisely, $f_{+}$and $f^{!}$send $\iota$-mixed $F$-complexes of weight $\geq w$ to those of weight $\geq w$. Similarly, $f_{!}$and $f^{+}$send $\iota$-mixed $F$-complexes of weight $\leq w$ to those of weight $\leq w, \mathbb{D}_{X}$ exchanges $\iota$-mixed $F$-complexes of weight $\leq w$ to $\geq-w$, and $\widetilde{\otimes}$ send $\iota$-mixed $F$-complexes of weight $\left(\geq w, \geq w^{\prime}\right)$ to $\geq w+w^{\prime}$.

(ii) Intermediate extension of an immersion preserves pure $F$-complexes and weights.

Concerning part (i), for the convenience of the reader, we recall that, in the very special case where $Y=$ Spec $k$, the stability under $f_{+}$was already checked by dévissage in overconvergent $F$-isocrystals from Kedlaya's stability of weights (see $\left[\right.$ Car06, 8.3.4] ${ }^{(1)}$ ). The relative version had been missing: as Kedlaya

\footnotetext{
(1) In fact, the compatibility with Frobenius of the comparison between push-forward and rigid cohomology was implicitly used. A proof of this compatibility is given in [Abe13]
} 
remarked in [Ked06, 5.3.3], one had at least to work with a theory admitting Grothendieck's six operations, which is only possible in the theory of arithmetic $\mathcal{D}$-modules. To check part (i), we follow some ideas appearing in Deligne's proof of the stability under push-forwards of [Del80], which leads us to the relative 1 dimensional case. To tackle this relative 1 dimensional case, specially concerning the stability of mixedness, one needs to study thoroughly the monodromy filtration associated to a log convergent isocrystal (this is section 3 of our work). One also notices that any proofs here do not use [Ked06]. Moreover, the part (ii) is completely new in $p$-adic cohomology theory.

Let us describe the contents of the paper. We restrict our attention to the category of realizable varieties, the full subcategory of the category of varieties over $k$ consisting of $Y$ such that there exists an immersion into a proper and smooth formal scheme over $\mathcal{V}$. We recall that quasi-projective varieties are realizable. In this introduction, let us simply call these objects, varieties.

In $\S 1$, we construct the coefficient theory using the foundational results of overholonomic modules. Even though the main ideas had already been provided, with the definition of a new t-structure here, the main theme of the first section is to extend some properties of the theory of arithmetic $\mathcal{D}$-modules and, at the same time, to make it in a more usable form. Let $Y$ be a variety. First, we recall the construction of the category $F$ - $D_{\text {ovhol }}^{\mathrm{b}}(Y / K)$ of overholonomic $F$-complexes over $Y / K$ and the formalism of the six operations for these coefficients. We then introduce a new t-structure on this category whose heart is denoted by $F$-Ovhol $(Y / K)$ and which may be seen as the category of arithmetic $\mathcal{D}$-modules over $Y / K$ (even though they are complexes and not modules). When $Y$ is smooth, we construct a fully faithful subcategory $F$-Isoc ${ }^{\dagger \dagger}(Y / K)$ of $F$-Ovhol $(Y / K)$ which is equivalent to the category of overconvergent $F$-isocrystals over $Y / K$ (the latter are the coefficients of the rigid cohomology). In the spirit of the Riemann-Hilbert correspondence, $F$-Ovhol $(Y / K)$ (resp. $F$-Isoc $\left.{ }^{\dagger \dagger}(Y / K)\right)$ is a $p$-adic analogue of the category of perverse sheaves (resp. smooth perverse sheaves) over $Y$. We proceed to check several necessary properties for being six operations formalism. We conclude the section by defining the intermediate extension as in the context of $\mathcal{D}$-modules over complex varieties. We also check that the analogous properties concerning irreducibility hold in our context. This enables us to define the $p$-adic intersection cohomology for realizable varieties, which is an advantage of using arithmetic $\mathcal{D}$-modules.

We fix an isomorphism $\iota: \overline{\mathbb{Q}}_{p} \cong \mathbb{C}$. In $\S 2$, we define the notion of $\iota$-mixedness as Deligne does for the $\ell$-adic cohomology in [Del80]. More precisely, in the spirit of the Riemann-Hilbert correspondence, the $\iota$ purity of an object in $F$-Isoc ${ }^{\dagger \dagger}(Y / K)$ is by definition a pointwise property (with the natural definition over a point) and the category of $\iota$-mixed objects of $F$-Isoc ${ }^{\dagger \dagger}(Y / K)$ is the full subcategory generated by extensions of $\iota$-pure objects. Next, by dévissage in overconvergent $F$-isocrystals, we extend naturally the notion of $\iota$-mixedness to $F$ - $D_{\text {ovhol }}^{\mathrm{b}}(Y / K)$. At the end of the section, we estimate the weight of the cohomology on curves, using the methods developed in [AM11] by the first author together with Marmora.

The aim of $\S 3$ is to prove the following preliminary results on the $\iota$-mixedness stability. Let $X$ be a proper smooth variety, $Z$ be a strict normal crossing divisor of $X$ and $j: Y:=X \backslash Z \rightarrow X$ be the open immersion. Let $\mathcal{G}$ be a $\log$-convergent $F$-isocrystal on $X$ with logarithmic poles along $Z$ which possesses nilpotent residues and let $\mathcal{E}$ be the object of $F$-Isoc $\operatorname{Is}^{\dagger \dagger}(Y / K)$ induced by restriction. If $\mathcal{E}$ is $\iota$-mixed, then we show that $j_{!}(\mathcal{E})$ is $\iota$-mixed as well. Moreover, when $Z$ is smooth and $\mathcal{E}$ is $\iota$-pure, we prove that the weight of $j_{!}(\mathcal{E})$ is less than or equal to that of $\mathcal{E}$. The theorem follows from the study of the monodromy filtration given by the nilpotent residue morphisms of $\mathcal{G}$.

In the last section $\S 4$, we prove the stability of $\iota$-mixedness and weights under the six operations. Roughly speaking, we reduce to the case treated in the previous section by using Kedlaya's semistable reduction theorem (see [Ked11]). Since we need to take special care of the boundary in p-adic cohomology theory compared to the case of $\ell$-adic theory, the proof of this theorem is slight involved. Finally, by using NootHuyghe's Fourier transform ([NH04]), we establish the stability of the $\iota$-purity under intermediate extension, which is a $p$-adic analogue of Gabber's purity theorem. We conclude the section with a few applications: the weight filtration for an $\iota$-mixed object of $F$-Ovhol $(Y / K)$, the semi-simplicity of $\iota$-mixed $F$-complexes after forgetting Frobenius structures, and some $\ell$-independence type results. 


\section{Acknowledgment}

The first author (T.A.) would like to thank F. Trihan for asking a question whose answer is nothing but Corollary 4.3.13. He would also like to thank O. Gabber for sending the original proof of his purity theorem, and giving some opinion of his against what (T.A.) was thinking. Finally (T.A.) would like to thank T. Saito for his continuous encouragement and numerable suggestions. This work was supported by Grant-in-Aid for Research Activity Start-up 23840006.

The second author (D.C.) is grateful to A. Pál. Pál noticed that Fujiwara-Gabber's $\ell$-independence in the form of Theorem 4.3.11 was a consequence of a $p$-adic version of Gabber's purity theorem. His remark gave one motivation of the second author to undertake this work on weight theory.

\section{Notation and convention}

Throughout this paper, $\mathcal{V}$ is a complete discrete valuation ring with mixed characteristic $(0, p), K$ is its field of fractions, $k$ is its residue field which is assumed to be perfect. We suppose that there exists a lifting of the Frobenius of $k$ to $\mathcal{V}$. In principle, we denote formal schemes by using script fonts (e.g. $\mathcal{X}$ ), and the special fiber is denoted by the corresponding capital letter (e.g. $X$ ). A $k$-variety is a separated scheme of finite type over $k$. We remark that without loss of generality, one could assume varieties over $k$ are reduced if needed. We often use $\star$ to abbreviate when it is obvious what should be put and do not want to introduce too many notations. If there is no risk of confusion, we sometimes use $(-)$ by meaning respectively. For example " $p^{(\prime)} \cong q^{(\prime)}$ " means " $p \cong q\left(\right.$ resp. $\left.p^{\prime} \cong q^{\prime}\right)$ ".

In this paper, we consider one of the following situations:

(A) Let $q=p^{s}$ be a power of $p$, and fix a lifting $\sigma$ of the $s$-th Frobenius $F$ of $k$ to $\mathcal{V}$ and $K$. (§1)

(B) We are in situation (A), and moreover, we assume that $k$ is a finite field with $q$ elements, and $F$ is the $s$-th Frobenius. We fix an isomorphism $\iota: \overline{\mathbb{Q}}_{p} \cong \mathbb{C}$. (Except for $\S 1$ )

\section{Preliminaries on arithmetic $\mathcal{D}$-modules}

Throughout this section, we consider situation (A) in Notation and convention.

\subsection{Arithmetic $\mathcal{D}$-modules over frames and couples}

In this subsection, we fix terminologies, and recall the definitions of six functors in the theory of arithmetic $\mathcal{D}$-modules. Fundamental additional properties are established from the next subsection.

Definition 1.1.1. We define the following categories:

(i) A frame $(Y, X, \mathcal{P})$ is the data consisting of a separated and smooth formal scheme $\mathcal{P}$ over $\mathcal{V}$, a closed subvariety $X$ of $P$, an open subscheme $Y$ of $X$. A morphism of frames $u=(b, a, f):(Y, X, \mathcal{P}) \rightarrow\left(Y^{\prime}, X^{\prime}, \mathcal{P}^{\prime}\right)$ is the data consisting of morphisms $b: Y^{\prime} \rightarrow Y, a: X^{\prime} \rightarrow X, f: \mathcal{P}^{\prime} \rightarrow \mathcal{P}$ such that $f$ induces the other ones.

(ii) An l.p. frame $(2)(Y, X, \mathcal{P}, \mathcal{Q})$ is the data consisting of a proper and smooth formal scheme $\mathcal{Q}$ over $\mathcal{V}$, an open formal subscheme $\mathcal{P}$ of $\mathcal{Q}$, a closed subvariety $X$ of $P$, an open subscheme $Y$ of $X$. A morphism of frames $u=(b, a, g, f):(Y, X, \mathcal{P}, \mathcal{Q}) \rightarrow\left(Y^{\prime}, X^{\prime}, \mathcal{P}^{\prime}, \mathcal{Q}^{\prime}\right)$ is the data consisting of morphisms $b: Y^{\prime} \rightarrow Y$, $a: X^{\prime} \rightarrow X, g: \mathcal{P}^{\prime} \rightarrow \mathcal{P}, f: \mathcal{Q}^{\prime} \rightarrow \mathcal{Q}$ such that $f$ induces the other ones. A morphism of l.p. frames is said to be complete if $a$ is proper.

Definition 1.1.2. We define the category of $\operatorname{couples}^{(3)}$ as follows:

- An object $(Y, X)$ is the data consisting of a $k$-variety $X$ and an open subscheme $Y$ of $X$ such that there exists an l.p. frame of the form $(Y, X, \mathcal{P}, \mathcal{Q})$.

\footnotetext{
${ }^{(2)}$ Abbreviation of "locally proper frame".

${ }^{(3)}$ In [Car12a], couples are called "properly realizable couples". Contrary to ibid., we only use this type of couples, so we simplify the name.
} 
- A morphism $\left(Y^{\prime}, X^{\prime}\right) \rightarrow(Y, X)$ of couples is a data $(b, a)$ consisting morphisms of $k$-varieties $a: X^{\prime} \rightarrow X$ and $b: Y^{\prime} \rightarrow Y$ such that $b$ is induced by $a$. The morphism $u$ is said to be complete if $a$ is proper. Let $\mathrm{P}$ be a property of morphisms of schemes. We say ${ }^{(4)}$ that $u$ is $c$-P if $u$ is complete and $b$ satisfies $\mathrm{P}$. (e.g. $u$ is a c-immersion if $u$ is proper and $b$ is an immersion.)

For a couple $(Y, X)$, we sometimes denote by $\mathbb{Y}$ using the bold font corresponding to the first data of the couple.

Let $u=: \mathbb{Y} \rightarrow \mathbb{Y}^{\prime}$ be a morphism of couples. By definition, there exist some l.p. frames of the form $(Y, X, \mathcal{P}, \mathcal{Q})$ and $\left(Y^{\prime}, X^{\prime}, \mathcal{P}^{\prime}, \mathcal{Q}^{\prime}\right)$ such that $\mathbb{Y}=(Y, X)$ and $\mathbb{Y}^{\prime}=\left(Y^{\prime}, X^{\prime}\right)$. By replacing $\mathcal{Q}$ by $\mathcal{Q} \times \mathcal{Q}^{\prime}$ (resp. $\mathcal{P}$ by $\left.\mathcal{P} \times \mathcal{P}^{\prime}\right)$ if necessary, we check that there exists a morphism of l.p. frames $(b, a, g, f):(Y, X, \mathcal{P}, \mathcal{Q}) \rightarrow$ $\left(Y^{\prime}, X^{\prime}, \mathcal{P}^{\prime}, \mathcal{Q}^{\prime}\right)$ of $u$, namely a morphism of l.p. frames such that $u=(b, a)$. Moreover, we may take $g$ and $f$ to be smooth. When $u$ is complete, we can even choose $g, f$ to be proper.

Definition 1.1.3. The category of realizable varieties is the full subcategory of the category of varieties over $k$ consisting of $X$ such that there exists an immersion into a proper and smooth formal scheme over $\mathcal{V}$.

1.1.4. Let $\mathcal{A}$ be an additive category endowed with an additive endofunctor $F^{*}: \mathcal{A} \rightarrow \mathcal{A}$. We define the category $F-\mathcal{A}$ of $F$-objects of $\mathcal{A}$ as follows: $F$-objects consist of $(\mathcal{E}, \Phi)$ where $\mathcal{E}$ is an object of $\mathcal{A}$, and $\Phi: \mathcal{E} \stackrel{\sim}{\rightarrow} F^{*} \mathcal{E}$ is an isomorphism of $\mathcal{E}$. Later, we take $F$ to be the Frobenius pull-back, and $\Phi$ is called the Frobenius structure. A morphism of $F$-objects is a morphism in $\mathcal{A}$ commuting with Frobenius structures. We have a faithful additive functor $\varrho: F-\mathcal{A} \rightarrow \mathcal{A}$ defined by forgetting Frobenius structures. If $\mathcal{A}$ is abelian, $F-\mathcal{A}$ is abelian as well.

Now, let $\mathcal{T}$ be a triangulated category. Assume given an additive endofunctor $F^{*}$ of $\mathcal{T}$. The category $F-\mathcal{T}$ has a shift functor in an obvious way, and a triangle in $F-\mathcal{T}$ is said to be distinguished if it is distinguished triangle after we forget Frobenius structures. We simply say triangle instead of distinguished triangle for the rest of the paper. We caution, however, that $F-\mathcal{T}$ is not triangulated in general. More precisely, it only satisfies (TR1), (TR2) of [Har66].

1.1.5. Before defining some notions, the notion of overholonomic complexes is defined in [Car09b, 3.1], which we do not recall here. The derived category of overholonomic $\mathcal{D}_{\mathcal{X}, \mathbb{Q}^{-}}^{\dagger}$-complexes is denoted by $D_{\text {ovhol }}^{\mathrm{b}}(\mathcal{X})$. Let $\mathcal{X}$ be a smooth formal scheme over $\mathcal{V}$, and $Z$ be a closed subscheme of $X$. Then the localization functor $\mathbb{R} \underline{\Gamma}_{Z}^{\dagger}: D_{\text {ovhol }}^{\mathrm{b}}(\mathcal{X}) \rightarrow D_{\text {ovhol }}^{\mathrm{b}}(\mathcal{X})$ is defined in [Car04, 2.2] (see also [Car09b, 1.15]). We note that Berthelot defined a similar functor in [Ber02], but these functors are not known to coincide unless $Z$ is a divisor of $X$. There exists a functorial cone for the homomorphism $\mathbb{R} \underline{\Gamma}_{Z}^{\dagger} \rightarrow$ id by [Car12c, 4.4.3], which is denoted by $\left({ }^{\dagger} Z\right)$. When we are given two closed subschemes $Z_{1}, Z_{2}$ of $X$, we have a canonical isomorphism $\mathbb{R} \underline{\Gamma}_{Z_{1}}^{\dagger} \circ \mathbb{R} \underline{\Gamma}_{Z_{2}}^{\dagger} \cong \mathbb{R} \underline{\Gamma}_{Z_{1} \cap Z_{2}}^{\dagger}$ by [Car04, 2.2.8]. Other important properties of the functor are that when we have a closed immersion of smooth formal schemes $i: \mathcal{Z} \hookrightarrow \mathcal{X}$, then canonically $i_{+} \circ i^{!} \cong \mathbb{R} \underline{\Gamma}_{Z}^{\dagger}$ (cf. [Car04, 3.1.12]), and if a coherent module $\mathcal{E}$ is supported on $Z$, then the canonical homomorphism $\mathbb{R}_{Z}^{\dagger}(\mathcal{E}) \rightarrow \mathcal{E}$ is an isomorphism (cf. [Car04, 2.2.9]).

Definition. (i) Let $(Y, X, \mathcal{P})$ be a frame. We define the category $D_{\text {ovhol }}^{\mathrm{b}}(Y, \mathcal{P} / K)$ to be the full subcategory of $D_{\text {ovhol }}^{\mathrm{b}}\left(\mathcal{D}_{\mathcal{P}, \mathbb{Q}}^{\dagger}\right)$ consisting of complexes $\mathcal{E}$ such that there exists an isomorphism of the form $\mathcal{E} \stackrel{\sim}{\rightarrow} \underline{\Gamma}_{Y}^{\dagger}(\mathcal{E}):=$ $\mathbb{R} \underline{\Gamma}_{X}^{\dagger} \circ\left({ }^{\dagger} X \backslash Y\right)(\mathcal{E})$ (cf. [Car07, 3.2.1]). The category is triangulated.

(ii) Now, let $\mathbb{Y}$ be a couple (cf. 1.1.2). Take an l.p. frame $(Y, X, \mathcal{P}, \mathcal{Q})$ of $\mathbb{Y}$. By using Lemma [Car12a, 2.5], we can check that the category $D_{\text {ovhol }}^{\mathrm{b}}(Y, \mathcal{P} / K)$ does not depend, up to a canonical equivalence, on the choices of $\mathcal{P}$ and $\mathcal{Q}$ (i.e. this only depends on $\mathbb{Y}=(Y, X)$ which is fixed). Hence, we can denote $D_{\text {ovhol }}^{\mathrm{b}}(Y, \mathcal{P} / K)$ by $D_{\text {ovhol }}^{\mathrm{b}}(\mathbb{Y} / K)$. Its objects are called overholonomic complexes of arithmetic $\mathcal{D}$-modules on $\mathbb{Y}$, or simply overholonomic complexes on $\mathbb{Y}$.

(iii) Finally, let us consider Frobenius structure. On the category $D_{\text {ovhol }}^{\mathrm{b}}(Y, \mathcal{P} / K)$, we have the Frobenius pull-back functor. Thus the results of paragraph 1.1 .4 can be applied to get the category $F-D_{\text {ovhol }}^{\mathrm{b}}(Y, \mathcal{P} / K)$. We also have $F-D_{\text {ovhol }}^{\mathrm{b}}(\mathbb{Y} / K)$.

\footnotetext{
(4) The "c-" stands for "complete".
} 
Remark. Our category $F$ - $D_{\text {ovhol }}^{\mathrm{b}}(Y, \mathcal{P} / K)$ is an analog of the category of "Weil complexes" [KW01, VI.18], so even though it is not triangulated, it gives us a suitable formalism for the theory of weights.

1.1.6. Let $\mathbb{Y}$ be a couple. Choose an l.p. frame $(Y, X, \mathcal{P}, \mathcal{Q})$ of $\mathbb{Y}$.

(i) We have the dual functor

$$
\mathbb{D}_{Y, \mathcal{P}}: F-D_{\text {ovhol }}^{\mathrm{b}}(Y, \mathcal{P} / K) \rightarrow F-D_{\text {ovhol }}^{\mathrm{b}}(Y, \mathcal{P} / K)
$$

This is defined as follows: we denote by $\mathbb{D}_{\mathcal{P}}$ the $\mathcal{D}_{\mathcal{P}, \mathbb{Q}^{-}}^{\dagger}$ linear dual (e.g. see [Vir00] or [Ber02]). We define $\mathbb{D}_{Y, \mathcal{P}}$ by $\mathbb{D}_{Y, \mathcal{P}}(\mathcal{E}):=\mathbb{R} \underline{\Gamma}_{Y}^{\dagger} \circ \mathbb{D}_{\mathcal{P}}(\mathcal{E})$ for any $\mathcal{E} \in F$ - $D_{\text {ovhol }}^{\mathrm{b}}(Y, \mathcal{P} / K)$. The functor $\mathbb{D}_{Y, \mathcal{P}}$ only depends on the couple $\mathbb{Y}$ (more precisely, by using ${ }^{(5)}$ Lemma 1.2 .8 , we can check that the functor $\mathbb{D}_{Y, \mathcal{P}}$ is compatible with the canonical equivalences of categories of independence of 1.1.5). This functor, called the dual functor, is denoted by

$$
\mathbb{D}_{\mathbb{Y}}: F-D_{\text {ovhol }}^{\mathrm{b}}(\mathbb{Y} / K) \rightarrow F-D_{\text {ovhol }}^{\mathrm{b}}(\mathbb{Y} / K) \text {. }
$$

(ii) The tensor product $\stackrel{\mathbb{L}_{\otimes}^{\dagger}}{\mathcal{O}_{\mathcal{P}, \mathbb{Q}}}$ is defined on $F-D_{\text {ovhol }}^{\mathrm{b}}\left(\mathcal{D}_{\mathcal{P}, \mathbb{Q}}^{\dagger}\right)$. By [Car08], the bifunctor $\stackrel{\mathbb{L}^{\dagger}}{\mathcal{O}_{\mathcal{P}, \mathbb{Q}}}[-\operatorname{dim}(\mathcal{P})]$ is independent of the choice of $\mathcal{P}$ and is denoted by

$$
\widetilde{\otimes}_{\mathbb{Y}}: F-D_{\text {ovhol }}^{\mathrm{b}}(\mathbb{Y} / K) \times F-D_{\text {ovhol }}^{\mathrm{b}}(\mathbb{Y} / K) \rightarrow F-D_{\text {ovhol }}^{\mathrm{b}}(\mathbb{Y} / K) .
$$

This is called the twisted tensor product functor. For simplicity, we sometimes abbreviate $\widetilde{\otimes}_{\mathbb{Y}}$ by $\widetilde{\otimes}$.

(iii) We define the tensor product by

$$
(-) \otimes_{\mathbb{Y}}(-):=\mathbb{D}_{\mathbb{Y}}\left(\mathbb{D}_{\mathbb{Y}}(-) \widetilde{\otimes}_{\mathbb{Y}} \mathbb{D}_{\mathbb{Y}}(-)\right): D_{\text {ovhol }}^{\mathrm{b}}(\mathbb{Y} / K) \times D_{\text {ovhol }}^{\mathrm{b}}(\mathbb{Y} / K) \rightarrow D_{\text {ovhol }}^{\mathrm{b}}(\mathbb{Y} / K) .
$$

Remark. In the spirit of Riemann-Hilbert correspondence, our $-\widetilde{\otimes}-$ corresponds to $\mathbb{D}(\mathbb{D}(-) \otimes \mathbb{D}(-))$ in the theory of constructible sheaves, which justifies our notation.

1.1.7. Let $u: \mathbb{Y} \rightarrow \mathbb{Y}^{\prime}$ be a morphism of couples. We have the following cohomological functors.

(i) We have ordinary and extraordinary pull-back by $u$ :

$$
u^{+}, u^{!}: F-D_{\text {ovhol }}^{\mathrm{b}}\left(\mathbb{Y}^{\prime} / K\right) \rightarrow F-D_{\text {ovhol }}^{\mathrm{b}}(\mathbb{Y} / K) .
$$

They are constructed as follows: choose a morphism of l.p. frames $\widetilde{u}=(\star, \star, g, \star):(Y, X, \mathcal{P}, \mathcal{Q}) \rightarrow\left(Y^{\prime}, X^{\prime}, \mathcal{P}^{\prime}, \mathcal{Q}^{\prime}\right)$ of $u$. Then $\widetilde{u}^{!}:=\mathbb{R} \underline{\Gamma}_{Y}^{\dagger} \circ g^{!}$does not depend on the choices of l.p. frames (for instance, see Lemma [Car12a, 2.5]). We define $u^{!}$to be $\widetilde{u}^{!}$. Using this, we define $\widetilde{u}^{+}:=\mathbb{D}_{Y, \mathcal{P}} \circ \widetilde{u}^{!} \circ \mathbb{D}_{Y^{\prime}, \mathcal{P}^{\prime}}$, and $u^{+}:=\mathbb{D}_{\mathbb{Y}} \circ u^{!} \circ \mathbb{D}_{\mathbb{Y}^{\prime}}$.

(ii) If $u$ is complete (cf. Definition 1.1.2), we have the ordinary and extraordinary direct image by $u$ :

$$
u_{!}, u_{+}: F-D_{\mathrm{ovhol}}^{\mathrm{b}}(\mathbb{Y} / K) \rightarrow F-D_{\mathrm{ovhol}}^{\mathrm{b}}\left(\mathbb{Y}^{\prime} / K\right) .
$$

There are constructed as follows: choose a morphism of l.p. frames $\widetilde{u}=(\star, \star, g, \star):(Y, X, \mathcal{P}, \mathcal{Q}) \rightarrow\left(Y^{\prime}, X^{\prime}, \mathcal{P}^{\prime}, \mathcal{Q}^{\prime}\right)$ for $u$. Then we put $\widetilde{u}_{+}:=g_{+}$, which does not depend on the choices and define $u_{+}:=\widetilde{u}_{+}$. As usual, we put $\widetilde{u}_{!}:=\mathbb{D}_{Y^{\prime}, \mathcal{P}^{\prime}} \circ u_{+} \circ \mathbb{D}_{Y, \mathcal{P}}$, and $u_{!}:=\mathbb{D}_{\mathbb{Y}^{\prime}} \circ u_{+} \circ \mathbb{D}_{\mathbb{Y}}$.

1.1.8. Let us define some extra functors.

(i) Let $\mathbb{Y}=(Y, X)$ and $\mathbb{Y}^{\prime}=\left(Y^{\prime}, X^{\prime}\right)$ be couples. Let $\mathbb{Y}^{\prime \prime}:=\left(Y \times Y^{\prime}, X \times X^{\prime}\right)$, and denote by $p^{(\prime)}: \mathbb{Y}^{\prime \prime} \rightarrow$ $\mathbb{Y}^{(\prime)}$ the projections. We define the exterior tensor product by

$$
(-) \bigotimes_{K}(-):=p^{!}(-) \widetilde{\otimes}_{\mathbb{Y}^{\prime \prime}} p^{\prime !}(-): D_{\mathrm{ovhol}}^{\mathrm{b}}(\mathbb{Y} / K) \times D_{\text {ovhol }}^{\mathrm{b}}\left(\mathbb{Y}^{\prime} / K\right) \rightarrow D_{\text {ovhol }}^{\mathrm{b}}\left(\mathbb{Y}^{\prime \prime} / K\right) .
$$

(ii) Let $\mathbb{Y}=(Y, X)$ be a couple. Let $U$ be a subscheme of $Y, \bar{U}$ be the closure of $U$ in $X, \mathbb{U}:=(U, \bar{U})$ and $u: \mathbb{U} \rightarrow \mathbb{Y}$ be the canonical c-immersion. We put

$$
\mathbb{R} \underline{\Gamma}_{U}^{\dagger}:=u_{+} \circ u^{!}: F-D_{\text {ovhol }}^{\mathrm{b}}(\mathbb{Y} / K) \rightarrow F-D_{\text {ovhol }}^{\mathrm{b}}(\mathbb{Y} / K) .
$$

\footnotetext{
${ }^{(5)}$ We may check that this is not a vicious circle.
} 
Moreover, we put $\left({ }^{\dagger} U\right):=\mathbb{R} \underline{\Gamma}_{Y \backslash U}^{\dagger}$. If $U_{1}$ and $U_{2}$ are subschemes of $Y$, we have $\mathbb{R} \underline{\Gamma}_{U_{1}}^{\dagger} \circ \mathbb{R} \underline{\Gamma}_{U_{2}}^{\dagger} \stackrel{\sim}{\rightarrow} \mathbb{R} \underline{\Gamma}_{U_{1} \cap U_{2}}^{\dagger}$ and $\left({ }^{\dagger} U_{1}\right) \circ\left({ }^{\dagger} U_{2}\right) \stackrel{\sim}{\rightarrow}\left({ }^{\dagger} U_{1} \cup U_{2}\right)$. When $Z$ is closed subscheme of $Y$, we have the following localization triangle of functors:

$$
\mathbb{R} \underline{\Gamma}_{Z}^{\dagger} \rightarrow \mathrm{id} \rightarrow\left({ }^{\dagger} Z\right) \stackrel{+1}{\longrightarrow} .
$$

(iii) Finally, let $(Y, X, \mathcal{P})$ be a frame, and $\mathcal{E} \in D_{\text {ovhol }}^{\mathrm{b}}(Y, \mathcal{P} / K)$. For a subscheme $Y^{\prime}$ of $Y$, let $X^{\prime}$ be the closure of $Y^{\prime}$ in $X$ and $j=(\star, \star$, id $):\left(Y^{\prime}, X^{\prime}, \mathcal{P}\right) \rightarrow(Y, X, \mathcal{P})$ be the morphism of frames. We define $\mathcal{E} \|_{Y^{\prime}}$ to be $j^{!}(\mathcal{E})$.

1.1.9. We recall the following useful properties:

(i) Let $u: \mathbb{Y} \rightarrow \mathbb{Y}^{\prime}$ be a morphism of couples. From $\left[\right.$ Car08, 2.1.9], for any $\mathcal{E}, \mathcal{F} \in F$ - $D_{\text {ovhol }}^{\mathrm{b}}\left(\mathbb{Y}^{\prime} / K\right)$, we have the canonical isomorphism in $F$ - $D_{\text {ovhol }}^{\mathrm{b}}(\mathbb{Y} / K)$ :

$$
u^{!}\left(\mathcal{E} \widetilde{\otimes}_{\mathbb{Y}^{\prime}} \mathcal{F}\right) \stackrel{\sim}{\rightarrow} u^{!}(\mathcal{E}) \widetilde{\otimes}_{\mathbb{Y}} u^{!}(\mathcal{F}) .
$$

(ii) Let $u: \mathbb{Y} \rightarrow \mathbb{Y}^{\prime}$ be a c-proper morphism of couples. From the relative duality isomorphism together with [Abe13, 4.14], we get the isomorphism $u_{!} \stackrel{\sim}{\rightarrow} u_{+}$.

Lemma 1.1.10. Let $u: \mathbb{Y} \rightarrow \mathbb{Y}^{\prime}$ be a complete morphism of couples. We have adjoint pairs $\left(u^{+}, u_{+}\right)$and $\left(u_{!}, u^{!}\right)$.

Proof. By transitivity with the composition, we reduce to the case where $u$ is a c-open immersion and $u$ is c-proper. In the first case, we may check easily that we have adjoint pairs $\left(u^{+}, u_{+}\right)$(and then $\left(u_{!}, u^{!}\right)$by duality). In the second case, from the relative duality isomorphism, we need to check that we have adjoint pairs $\left(u_{+}, u^{!}\right)$, which follows from [Vir04].

\section{2 t-structures}

In this subsection, we introduce a t-structure on the triangulated category $F$ - $D_{\text {ovhol }}^{\mathrm{b}}(\mathbb{Y} / K)$ for a couple $\mathbb{Y}$. The heart of this t-structure is an analogue of the category of holonomic $\mathcal{D}$-modules for the theory of algebraic $\mathcal{D}$-modules.

Let $\mathbb{Y}=(Y, X)$ be a couple (cf. Definition 1.1.2). Choose an 1.p. frame $(Y, X, \mathcal{P}, \mathcal{Q})$ of $\mathbb{Y}$. Let $Z$ be a closed subvariety of $P$ (the special fiber of $\mathcal{P}$ following our convention) so that $Z:=X \backslash Y$. We set $\mathcal{U}:=\mathcal{P} \backslash Z$. Let us denote by $D^{\leq 0}\left(\mathcal{D}_{\mathcal{U}, \mathbb{Q}}^{\dagger}\right)$ (resp. $\left.D^{\geq 0}\left(\mathcal{D}_{\mathcal{U}, \mathbb{Q}}^{\dagger}\right)\right)$ the strictly full subcategory of $D^{\mathrm{b}}\left(\mathcal{D}_{\mathcal{U}, \mathbb{Q}}^{\dagger}\right)$ consisting of complexes $\mathcal{E}$ such that, for any $j \geq 1$ (resp. for any $j \leq-1$ ), we have $\mathcal{H}^{j}(\mathcal{E})=0$. We denote by $\tau_{\leq 0}: D^{\mathrm{b}}\left(\mathcal{D}_{\mathcal{U}, \mathbb{Q}}^{\dagger}\right) \rightarrow D^{\leq 0}\left(\mathcal{D}_{\mathcal{U}, \mathbb{Q}}^{\dagger}\right)$ and $\tau_{\geq 0}: D^{\mathrm{b}}\left(\mathcal{D}_{\mathcal{U}, \mathbb{Q}}^{\dagger}\right) \rightarrow D^{\geq 0}\left(\mathcal{D}_{\mathcal{U}, \mathbb{Q}}^{\dagger}\right)$ the usual truncation functors.

Definition 1.2.1. (i) Let $D^{\leq 0}(Y, \mathcal{P} / K)$ (resp. $\left.D^{\geq 0}(Y, \mathcal{P} / K)\right)$ be the strictly full subcategory of $D_{\text {ovhol }}^{\mathrm{b}}(Y, \mathcal{P} / K)$ of complexes $\mathcal{E}$ such that $\left.\mathcal{E}\right|_{\mathcal{U}} \in D^{\leq 0}\left(\mathcal{D}_{\mathcal{U}, \mathbb{Q}}^{\dagger}\right)\left(\right.$ resp. $\left.\left.\mathcal{E}\right|_{\mathcal{U}} \in D^{\geq 0}\left(\mathcal{D}_{\mathcal{U}, \mathbb{Q}}^{\dagger}\right)\right)$.

(ii) Let $\tau_{\leq 0}^{(Y, \mathcal{P})}: D_{\text {ovhol }}^{\mathrm{b}}(Y, \mathcal{P} / K) \rightarrow D_{\text {ovhol }}^{\mathrm{b}}(Y, \mathcal{P} / K)$ and $\tau_{\geq 0}^{(Y, \mathcal{P})}: D_{\text {ovhol }}^{\mathrm{b}}(Y, \mathcal{P} / K) \rightarrow D_{\text {ovhol }}^{\mathrm{b}}(Y, \mathcal{P} / K)$ be the functors defined by $\tau_{\leq 0}^{(Y, \mathcal{P})}:=\left({ }^{\dagger} Z\right) \circ \tau_{\leq 0}, \tau_{\geq 0}^{(Y, \mathcal{P})}:=\left({ }^{\dagger} Z\right) \circ \tau_{\geq 0}$.

(iii) For any $n \in \mathbb{Z}$, we put $D^{\leq n}(Y, \mathcal{P} / K):=D^{\leq 0}(Y, \mathcal{P} / K)[-n], D^{\geq n}(Y, \mathcal{P} / K):=D^{\geq 0}(Y, \mathcal{P} / K)[-n]$, $\tau_{\leq n}^{(Y, \mathcal{P})}:=[-n] \circ \tau_{\leq 0}^{(Y, \mathcal{P})} \circ[n]$ and $\tau_{\geq n}^{(Y, \mathcal{P})}:=[-n] \circ \tau_{\geq 0}^{(Y, \mathcal{P})} \circ[n]$.

Remark 1.2.2. (i) When we can choose $Z$ to be the support of a divisor of $P$, the definitions of 1.2 .1 are not really new. Indeed, in that case we get $D^{\leq 0}(Y, \mathcal{P} / K)=D^{\leq 0}\left(\mathcal{D}_{\mathcal{P}}^{\dagger}\left({ }^{\dagger} Z\right)_{\mathbb{Q}}\right)\left(\right.$ resp. $D^{\geq 0}(Y, \mathcal{P} / K)=$ $D^{\geq 0}\left(\mathcal{D}_{\mathcal{P}}^{\dagger}\left({ }^{\dagger} Z\right)_{\mathbb{Q}}\right)$. Moreover, in that case, we simply have $\tau_{\leq 0}^{(Y, \mathcal{P})}=\tau_{\leq 0}$ and $\tau_{\geq 0}^{(Y, \mathcal{P})}=\tau_{\geq 0}$ (i.e. the functor $\left({ }^{\dagger} Z\right.$ ) is useless).

(ii) Now, suppose that $Z$ is a rational closed point of $\mathbb{P}_{k}^{2}$, that $\mathcal{P}=\mathcal{Q}=\widehat{\mathbb{P}}_{\mathcal{V}}^{2}, X=\mathbb{P}_{k}^{2}$, and recall that $Y:=X \backslash Z$. Since $\left({ }^{\dagger} Z\right)\left(\mathcal{O}_{\mathcal{P}, \mathbb{Q}}\right) \mid \mathcal{U} \stackrel{\sim}{\rightarrow} \mathcal{O}_{\mathcal{U}, \mathbb{Q}}$, we get $\left({ }^{\dagger} Z\right)\left(\mathcal{O}_{\mathcal{P}, \mathbb{Q}}\right) \in D^{\leq 0}(Y, \mathcal{P} / K) \cap D^{\geq 0}(Y, \mathcal{P} / K)$. However, by using the localization triangle with respect to $Z$ of $\mathcal{O}_{\mathcal{P}, \mathbb{Q}}$, we have $\tau_{\leq 0}\left((\dagger Z)\left(\mathcal{O}_{\mathcal{P}, \mathbb{Q}}\right)\right) \stackrel{\sim}{\rightarrow} \mathcal{O}_{\mathcal{P}, \mathbb{Q}}$, which is 
not in $D_{\text {ovhol }}^{\mathrm{b}}(Y, \mathcal{P} / K)$. This means that the functor $\left({ }^{\dagger} Z\right)$ is needed in the definition of $\tau_{>0}^{(Y, \mathcal{P})}$. Now, let $u$ be a lifting of the closed immersion $Z \hookrightarrow P$. We have $\mathbb{R} \underline{\Gamma}_{Z}^{\dagger}\left(\mathcal{O}_{\mathcal{P}, \mathbb{Q}}\right) \cong u_{+} u^{!}\left(\mathcal{O}_{\mathcal{P}, \mathbb{Q}}\right) \cong u_{+}(K)[-2]$. Using the localization triangle again, we have $\tau_{\geq 1}\left(\left({ }^{\dagger} Z\right)\left(\mathcal{O}_{\mathcal{P}, \mathbb{Q}}\right)\right) \stackrel{\sim}{\rightarrow} u_{+}(K)[-1]$. Hence, the object $\left({ }^{\dagger} Z\right)\left(\mathcal{O}_{\mathcal{P}, \mathbb{Q}}\right)$ is not a module in the usual sense.

Lemma 1.2.3. Let $\phi: \mathcal{E} \rightarrow \mathcal{F}$ be a homomorphism of $D_{\mathrm{ovhol}}^{\mathrm{b}}(Y, \mathcal{P} / K)$. Then, $\phi$ is an isomorphism if and only if $\left.\phi\right|_{\mathcal{U}}$ is.

Proof. Let $\mathcal{G}$ be a mapping cone of $\phi$, is an object of $D_{\text {ovhol }}^{\mathrm{b}}(Y, \mathcal{P} / K)$. Since $\phi \mid \mathcal{U}$ is an isomorphic, $\mathcal{G}$ is supported in $Z$. Thus, we have $\mathcal{G} \longleftarrow \mathbb{R}_{Z}^{\dagger}(\mathcal{G})$. But since $\mathcal{G}$ is an object of $D_{\text {ovhol }}^{\mathrm{b}}(Y, \mathcal{P} / K)$ we get $\mathcal{G} \stackrel{\sim}{\rightarrow}\left({ }^{\dagger} Z\right)(\mathcal{G})$. Thus, we have $\mathcal{G} \stackrel{\sim}{\rightarrow}\left({ }^{\dagger} Z\right)(\mathcal{G}) \stackrel{\sim}{\sim}\left({ }^{\dagger} Z\right)\left(\mathbb{R} \underline{\Gamma}_{Z}^{\dagger}(\mathcal{G})\right) \cong\left(\left({ }^{\dagger} Z\right) \circ \mathbb{R} \underline{\Gamma}_{Z}^{\dagger}\right)(\mathcal{G})=0$ where the last equality follows by the localization triangle 1.1.8 (ii).

Lemma 1.2.4. Let $\mathcal{E} \in D^{\leq n}(Y, \mathcal{P} / K)\left(\right.$ resp. $\mathcal{E} \in D^{\geq n}(Y, \mathcal{P} / K)$ ). The canonical homomorphism $\tau_{\leq n}^{(Y, \mathcal{P})}(\mathcal{E}) \rightarrow$ $\mathcal{E}$ (resp. $\mathcal{E} \rightarrow \tau_{\geq n}^{(Y, \mathcal{P})}(\mathcal{E})$ ) is an isomorphism. In particular, the essential image of $\tau_{\leq n}^{(Y, \mathcal{P})}$ (resp. $\tau_{\geq n}^{(Y, \mathcal{P})}$ ) is $D^{\leq n}(Y, \mathcal{P} / K)\left(\right.$ resp. $\left.D^{\geq n}(Y, \mathcal{P} / K)\right)$.

Proof. This follows from Lemma 1.2.3.

Proposition 1.2.5. The functors $\tau_{\star}^{(Y, \mathcal{P})}$ define a t-structure, called the canonical t-structure, on $D_{\text {ovhol }}^{\mathrm{b}}(Y, \mathcal{P} / K)$ :

(i) For any homomorphism $f: \mathcal{E} \rightarrow \mathcal{F}$ such that $\mathcal{E} \in D^{\leq 0}(Y, \mathcal{P} / K), \mathcal{F} \in D^{\geq 1}(Y, \mathcal{P} / K)$, we have $f=0$.

(ii) We have the inclusions $D^{\geq 1}(Y, \mathcal{P} / K) \subset D^{\geq 0}(Y, \mathcal{P} / K), D^{\leq 0}(Y, \mathcal{P} / K) \subset D^{\leq 1}(Y, \mathcal{P} / K)$.

(iii) For any $\mathcal{E} \in D_{\text {ovhol }}^{\mathrm{b}}(Y, \mathcal{P} / K)$, we have the distinguished triangle

$$
\tau_{\leq 0}^{(Y, \mathcal{P})}(\mathcal{E}) \rightarrow \mathcal{E} \rightarrow \tau_{\geq 1}^{(Y, \mathcal{P})}(\mathcal{E}) \stackrel{+1}{\longrightarrow} .
$$

Proof. Since $\tau_{\leq 0}^{(Y, \mathcal{P})}(\mathcal{F})$ has its support in $Z, \tau_{\leq 0}^{(Y, \mathcal{P})}(\mathcal{F})=0$. From Lemma 1.2.4, we get that $f$ factorizes through $\tau_{\leq 0}^{(Y, \mathcal{P})}(f)=0$. Hence we get the first assertion. The other ones are obvious.

Definition 1.2.6. We denote by $\operatorname{Ovhol}(Y, \mathcal{P} / K)$ the heart of the canonical t-structure on $D_{\text {ovhol }}^{\mathrm{b}}(Y, \mathcal{P} / K)$. We define for any integer $n$, the $n$-th cohomology functor $\mathcal{H}_{\mathrm{t}}^{n}: D_{\text {ovhol }}^{\mathrm{b}}(Y, \mathcal{P} / K) \rightarrow \operatorname{Ovhol}(Y, \mathcal{P} / K)$ by putting $\mathcal{H}_{\mathrm{t}}^{n}(\mathcal{E}):=\tau_{\leq 0}^{(Y, \mathcal{P})} \tau_{\geq 0}^{(Y, \mathcal{P})}(\mathcal{E}[n])$ for any $\mathcal{E} \in D_{\text {ovhol }}^{\mathrm{b}}(Y, \mathcal{P} / K)$.

Let $(Y, X, \mathcal{P}, \mathcal{Q})$ be an l.p. frame. An important example of object of $\operatorname{Ovhol}(Y, \mathcal{P} / K)$ is $\left({ }^{\dagger} Z\right) \mathcal{O}_{\mathcal{P}, \mathbb{Q}}$ where $Z:=X \backslash Y$.

Remark 1.2.7. (i) Let $\mathcal{E} \in D_{\text {ovhol }}^{\mathrm{b}}(Y, \mathcal{P} / K)$. We get a natural morphism $\mathcal{H}^{n}(\mathcal{E}) \rightarrow \mathcal{H}_{\mathrm{t}}^{n}(\mathcal{E})$, where $\mathcal{H}^{n}(\mathcal{E})$ is the usual $n$-th cohomology functor. When $Z$ can be chosen to be the support of a divisor of $P$, this morphism is an isomorphism. But in general this is not the case (e.g. see the example given in the second remark 1.2.2). However, the induced morphism

$$
\left({ }^{\dagger} Z\right) \circ \mathcal{H}^{n}(\mathcal{E}) \rightarrow \mathcal{H}_{\mathrm{t}}^{n}(\mathcal{E}),
$$

is an isomorphism since this is the case outside $Z$.

(ii) For any $n \in \mathbb{Z}, \mathcal{E} \in D_{\text {ovhol }}^{\mathrm{b}}(Y, \mathcal{P} / K)$, we have $\left.\mathcal{H}_{\mathrm{t}}^{n}(\mathcal{E})\right|_{\mathcal{U}} \stackrel{\sim}{\rightarrow} \mathcal{H}^{n}\left(\left.\mathcal{E}\right|_{\mathcal{U}}\right)$. Hence, from Lemma 1.2.3, $\mathcal{H}_{\mathrm{t}}^{n}(\mathcal{E})=0$ if and only if $\mathcal{H}^{n}\left(\left.\mathcal{E}\right|_{\mathcal{U}}\right)=0$.

(iii) When $Z$ can be chosen to be the support of a divisor of $P$, the category $\operatorname{Ovhol}(Y, \mathcal{P} / K)$ is simply the category of overholonomic $\mathcal{D}_{\mathcal{P}}^{\dagger}\left({ }^{\dagger} Z\right)_{\mathbb{Q}}$-modules, i.e. the (usual) heart of the category $D_{\text {ovhol }}^{\mathrm{b}}\left(\mathcal{D}_{\mathcal{P}}^{\dagger}\left({ }^{\dagger} Z\right)_{\mathbb{Q}}\right)$.

(iv) We remark that the category $\operatorname{Ovhol}(Y, \mathcal{P} / K)$ is also the strictly full subcategory of $D_{\text {ovhol }}^{\mathrm{b}}(Y, \mathcal{P} / K)$ consisting of complexes $\mathcal{E}$ such that $\mathcal{H}^{i}\left(\left.\mathcal{E}\right|_{\mathcal{U}}\right)=0$ for $i \neq 0$. By Lemma 1.2.4, the category Ovhol $(Y, \mathcal{P} / K)$ is also the strictly full subcategory of $D_{\text {ovhol }}^{\mathrm{b}}(Y, \mathcal{P} / K)$ of complexes $\mathcal{E}$ such that $\mathcal{H}_{\mathrm{t}}^{i}(\mathcal{E})=0$ for $i \neq 0$. By (1.2.7.1), this implies the inclusion $\operatorname{Ovhol}(Y, \mathcal{P} / K) \subset D^{\geq 0}\left(\mathcal{D}_{\mathcal{P}, \mathbb{Q}}^{\dagger}\right)$ (remark that with the example given in the second remark 1.2.2, the objects of $\operatorname{Ovhol}(Y, \mathcal{P} / K)$ are not in general modules). Indeed, by using MayerVietoris exact triangles and an induction on the number of divisors whose intersection is $Z$, we reduce to the case where $Z$ is a divisor. 
Next, we complete [Car11b, 4.2.3] with the following lemma:

Lemma 1.2.8. Let $u=(\mathrm{id}, \star, \star, \star):\left(Y, X^{\prime}, \mathcal{P}^{\prime}, \mathcal{Q}^{\prime}\right) \rightarrow(Y, X, \mathcal{P}, \mathcal{Q})$ be a complete morphism of l.p. frames.

1. For any $\mathcal{E} \in \operatorname{Ovhol}(Y, \mathcal{P} / K), \mathcal{E}^{\prime} \in \operatorname{Ovhol}\left(Y, \mathcal{P}^{\prime} / K\right)$, for any $n \in \mathbb{Z} \backslash\{0\}$,

$$
\mathcal{H}_{\mathrm{t}}^{n}\left(u^{!}\left(\mathcal{E}^{\prime}\right)\right)=0, \quad \mathcal{H}_{\mathrm{t}}^{n}\left(u_{+}(\mathcal{E})\right)=0 .
$$

2. The functors $\mathcal{H}_{\mathrm{t}}^{0} u^{!}$and $\mathcal{H}_{\mathrm{t}}^{0} u_{+}$(resp. $u^{!}$and $u_{+}$) induce equivalence of categories between $\operatorname{Ovhol}(Y, \mathcal{P} / K)$ and $\operatorname{Ovhol}\left(Y, \mathcal{P}^{\prime} / K\right)$ (resp. between $F$ - $D_{\text {ovhol }}^{\mathrm{b}}(Y, \mathcal{P} / K)$ and $F$ - $\left.D_{\text {ovhol }}^{\mathrm{b}}\left(Y, \mathcal{P}^{\prime} / K\right)\right)$.

3. We have canonical isomorphisms $u_{!} \stackrel{\sim}{\rightarrow} u_{+}$and $u^{!} \stackrel{\sim}{\rightarrow} u^{+}$.

Proof. By Remark 1.2.7, the two first statements follows by [Car11b, 4.2.3]. Concerning the last one, we remark that the isomorphism $u_{!} \stackrel{\sim}{\rightarrow} u_{+}$is equivalent to the other one $u^{!} \stackrel{\sim}{\rightarrow} u^{+}$. Moreover, by transitivity, we come down to the case where $g$ is proper and is an open immersion. In the proper case we have $u_{!} \stackrel{\sim}{\rightarrow} u_{+}$, and in the open immersion case, we have $u^{!} \stackrel{\sim}{\rightarrow} u^{+}$.

Definition 1.2.9. (i) Let $\mathbb{Y}$ be a couple. Take an l.p. frame $(Y, X, \mathcal{P}, \mathcal{Q})$ of $\mathbb{Y}$. By Lemma 1.2.8, the tstructure of $D_{\mathrm{ovhol}}^{\mathrm{b}}(Y, \mathcal{P} / K)$ is compatible with canonical equivalence of categories, and independent on the choices of $\mathcal{P}$ and $\mathcal{Q}$ (cf. Definition 1.1.5). This makes $D_{\text {ovhol }}^{\mathrm{b}}(\mathbb{Y} / K)$ a triangulated category with t-structure. Its heart is denoted by $\operatorname{Ovhol}(\mathbb{Y} / K)$. The objects in $\operatorname{Ovhol}(\mathbb{Y} / K)$ are called overholonomic arithmetic $\mathcal{D}$ modules on $\mathbb{Y}$, or overholonomic $\mathcal{D}_{\mathbb{Y}}^{\dagger}$-modules. For simplicity, we often call them $\mathcal{D}_{\mathbb{Y}}^{\dagger}$-modules, or modules on $\mathbb{Y}$.

(ii) Let $u: \mathbb{Y}^{\prime} \rightarrow \mathbb{Y}$ be a morphism of couples. We define two functors Ovhol( $\left.\mathbb{Y} / K\right) \rightarrow \operatorname{Ovhol}\left(\mathbb{Y}^{\prime} / K\right)$ by $u^{! 0}:=\mathcal{H}_{\mathrm{t}}^{0} \circ u^{!}$and $u^{+0}:=\mathcal{H}_{\mathrm{t}}^{0} \circ u^{+}$. When $u$ is complete, we define two functors Ovhol $\left(\mathbb{Y}^{\prime} / K\right) \rightarrow \operatorname{Ovhol}(\mathbb{Y} / K)$ by setting $u_{+}^{0}:=\mathcal{H}_{\mathrm{t}}^{0} \circ u_{+}$and $u_{!}^{0}:=\mathcal{H}_{\mathrm{t}}^{0} \circ u_{!}$.

(iii) Let $j:\left(Y^{\prime}, X^{\prime}\right) \rightarrow(Y, X)$ be a morphism of couples such that $X^{\prime} \rightarrow X$ is an immersion. We denote $j^{! 0}$ by $\left.\right|_{\left(Y^{\prime}, X^{\prime}\right)}$.

Remark 1.2.10. Let $j:\left(Y, X^{\prime}\right) \rightarrow(Y, X)$ be a morphism of couples such that $X^{\prime} \rightarrow X$ is an open immersion. Then a sequence $\mathcal{E}^{\prime} \rightarrow \mathcal{E} \rightarrow \mathcal{E}^{\prime \prime}$ in $\operatorname{Ovhol}((Y, X) / K)$ is t-exact if and only if so is the sequence $\left.\mathcal{E}^{\prime}\right|_{\left(Y, X^{\prime}\right)} \rightarrow$ $\left.\left.\mathcal{E}\right|_{\left(Y, X^{\prime}\right)} \rightarrow \mathcal{E}^{\prime \prime}\right|_{\left(Y, X^{\prime}\right)}$. Moreover, an object $\mathcal{E} \in \operatorname{Ovhol}(\mathbb{Y} / K)$ is 0 if and only if $\left.\mathcal{E}\right|_{\left(Y, X^{\prime}\right)}$ is 0 . In particular, the functor $\left.\right|_{\left(Y, X^{\prime}\right)}: \operatorname{Ovhol}((Y, X) / K) \rightarrow \operatorname{Ovhol}\left(\left(Y, X^{\prime}\right) / K\right)$ is faithful.

Definition 1.2.11. Let $\mathbb{Y}, \mathbb{Y}^{\prime}$ be two couples and $\phi: D_{\text {ovhol }}^{\mathrm{b}}(\mathbb{Y} / K) \rightarrow D_{\text {ovhol }}^{\mathrm{b}}\left(\mathbb{Y}^{\prime} / K\right)$ be a functor. We say that $\phi$ is left $t$-exact (resp. right $t$-exact, resp. $t$-exact) if, for any $\mathcal{E} \in \operatorname{Ovhol}(Y, \mathcal{P} / K)$ and any integer $n \in \mathbb{Z}$ such that $n \leq-1$ (resp. $n \geq 1$, resp. $n \neq 0$ ), we have $\mathcal{H}_{\mathrm{t}}^{n} \circ \phi\left(\mathcal{E}^{\prime}\right)=0$.

1.2.12. Let $\left(Y^{(\prime)}, X^{(\prime)}\right) \rightarrow\left(Y^{\prime \prime}, X^{\prime \prime}\right)$ be morphisms of couples. We denote $(Y, X) \times_{\left(Y^{\prime \prime}, X^{\prime \prime}\right)}\left(Y^{\prime}, X^{\prime}\right):=$ $\left(Y \times_{Y^{\prime \prime}} Y^{\prime}, X \times_{X^{\prime \prime}} X^{\prime}\right)$. Let $\left\{\mathbb{Y}_{i}\right\}_{i \in I}$ be a c-open covering of $\mathbb{Y}$, namely, we have the c-open immersions $\left(Y_{i}, X_{i}\right)=\mathbb{Y}_{i} \rightarrow \mathbb{Y}$ such that $\left\{Y_{i}\right\}_{i \in I}$ is an open covering of $Y$. We put $\mathbb{Y}_{i j}:=\mathbb{Y}_{i} \times_{\mathbb{Y}} \mathbb{Y}_{j}$ and $\mathbb{Y}_{i j k}:=\mathbb{Y}_{i j} \times_{\mathbb{Y}} \mathbb{Y}_{k}$. For any $i, j, k \in I$, we denote by $u_{i}: \mathbb{Y}_{i} \rightarrow \mathbb{Y}, u_{i j}: \mathbb{Y}_{i j} \rightarrow \mathbb{Y}_{i}, v_{i j}:=u_{i} \circ u_{i j}, u_{i j k}: \mathbb{Y}_{i j k} \rightarrow \mathbb{Y}_{i j}$ the induced c-open immersions.

Now, we define $\operatorname{Ovhol}\left(\left\{\mathbb{Y}_{i}\right\}_{i \in I} / K\right)$ to be the category whose objects are the data of objects $\mathcal{E}_{i} \in$ $\operatorname{Ovhol}\left(\mathbb{Y}_{i} / K\right)$ endowed with isomorphisms of the form $\theta_{j i}: u_{i j}^{! 0}\left(\mathcal{E}_{i}\right) \stackrel{\sim}{\rightarrow} u_{j i}^{! 0}\left(\mathcal{E}_{j}\right)$ which satisfy the cocycle condition $u_{i j k}^{! 0}\left(\theta_{k i}\right)=u_{j k i}^{! 0}\left(\theta_{k j}\right) \circ u_{i j k}^{! 0}\left(\theta_{i j}\right)$, for any $i, j, k \in I$. A morphism $\left(\mathcal{E}_{i}, \theta_{i j}\right) \rightarrow\left(\mathcal{E}_{i}^{\prime}, \theta_{i j}^{\prime}\right)$ in $\operatorname{Ovhol}\left(\left\{\mathbb{Y}_{i}\right\}_{i \in I} / K\right)$ is a collection of morphisms $f_{i}: \mathcal{E}_{i} \rightarrow \mathcal{E}_{i}^{\prime}$ in $\operatorname{Ovhol}\left(\mathbb{Y}_{i} / K\right)$ such that $u_{j i}^{! 0}\left(f_{i}\right) \circ \theta_{i j}=\theta_{i j}^{\prime} \circ u_{i j}^{! 0}\left(f_{j}\right)$, for any $i, j \in I$.

Lemma (Gluing). The canonical functor $\operatorname{Ovhol}(\mathbb{Y} / K) \rightarrow \operatorname{Ovhol}\left(\left\{\mathbb{Y}_{i}\right\}_{i \in I} / K\right)$ is an equivalence of categories.

Proof. By the quasi-compactness of $Y$, we may assume $I$ to be a finite set. We can construct a canonical quasi-inverse functor as follows: let $\left\{\mathcal{E}_{i}, \theta_{i j}\right\} \in \operatorname{Ovhol}\left(\left(\mathbb{Y}_{i}\right)_{i \in I} / K\right)$. We denote by $d_{1}, d_{2}: \prod_{i \in I} u_{i+}^{0} \mathcal{E}_{i} \rightarrow$ $\prod_{i, j \in I} v_{i j+}^{0} u_{i j}^{! 0}\left(\mathcal{E}_{i}\right)$ the morphisms such that $d_{1}$ is induced by $u_{i+}^{0} \mathcal{E}_{i} \rightarrow v_{i j+}^{0} u_{i j}^{! 0}\left(\mathcal{E}_{i}\right)$ and $d_{2}$ is induced by $u_{j+}^{0} \mathcal{E}_{j} \rightarrow v_{j i+}^{0} u_{j i}^{! 0}\left(\mathcal{E}_{j}\right) \underset{\theta_{i j}}{\stackrel{\sim}{\longrightarrow}} v_{i j+}^{0} u_{i j}^{! 0}\left(\mathcal{E}_{i}\right)$. Then the canonical quasi-inverse functor is by definition the kernel of $d_{1}-d_{2}$. 
1.2.13. Recall the situation of paragraph 1.1.4. Let $\mathcal{T}$ be a triangulated category with t-structure $\tau_{*}$. Assume given an additive endofunctor $F^{*}$ of $\mathcal{T}$ which is assumed to be t-exact. The truncation functor $\tau_{*}$ lifts to a functor from $F-\mathcal{T}$ to itself since $F^{*}$ is assumed to be t-exact. By construction, $\tau_{*}$ commutes with $\varrho$. As usual, we put $\mathcal{H}^{n}:=\tau_{\leq n} \circ \tau_{\geq n}$, and are able to define the "heart" of $F-\mathcal{T}$, in an obvious manner. This heart is nothing but $F$-Heart $(\mathcal{T})$, and in particular, it is abelian.

Now, the Frobenius pull-back functor on the category $D_{\text {ovhol }}^{\mathrm{b}}(Y, \mathcal{P} / K)$ is t-exact. Hence, we may apply the abstract non-sense above, and get that the heart of $F-D_{\text {ovhol }}^{\mathrm{b}}(Y, \mathcal{P} / K)\left(\operatorname{resp} . F-D_{\text {ovhol }}^{\mathrm{b}}(\mathbb{Y} / K)\right)$ is $F$-Ovhol $(Y, \mathcal{P} / K)($ resp. $F$-Ovhol $(\mathbb{Y} / K))$. The category $F$-Ovhol $(\mathbb{Y} / K)$ is noetherian and artinian. Indeed, we reduce to the case where $Y=X=P$ in which case it follows by [Ber02, 5.4.3].

1.2.14. Let $\mathbb{Y}=(Y, X)$ be a couple such that $Y$ is smooth. Let $Z:=X \backslash Y$. When there exists a divisor $W$ of $P$ such that $Z=W \cap X$, a strictly full subcategory $\operatorname{Isoc}^{\dagger \dagger}(Y, \mathcal{P} / K)$ of the category of coherent $\mathcal{D}_{\mathcal{P}}^{\dagger}\left({ }^{\dagger} Z\right)_{\mathbb{Q}}$-modules with support in $X$ is defined in [Car11a]. Moreover, we have the equivalence

$$
\operatorname{sp}_{+}: \operatorname{Isoc}^{\dagger}(Y, X / K) \stackrel{\sim}{\rightarrow} \operatorname{Isoc}^{\dagger \dagger}(Y, \mathcal{P} / K) .
$$

We may endow with Frobenius structure: $F$-Isoc ${ }^{\dagger \dagger}(Y, \mathcal{P} / K)$ is a full subcategory of $F$-Ovhol $(Y, \mathcal{P} / K)$ (cf. [CT12]), and the above equivalence commutes with Frobenius structures, i.e. induces the equivalence $\mathrm{sp}_{+}: F-\operatorname{Isoc}^{\dagger}(Y, X / K) \stackrel{\sim}{\longrightarrow} F-\operatorname{Isoc}^{\dagger \dagger}(Y, \mathcal{P} / K)$.

Let us generalize this latter equivalence with Frobenius structures (because in our context we need to stay with overholonomic complexes) to arbitrary $Z$. We define the category $F$ - $\operatorname{Isoc}^{\dagger \dagger}(Y, \mathcal{P} / K)$ to be the strictly full subcategory of $F$-Ovhol $(Y, \mathcal{P} / K)$ consisting of objects $\mathcal{E}$ such that $\left.\mathcal{E}\right|_{\mathcal{U}} \in F$-Isoc ${ }^{\dagger \dagger}(Y, \mathcal{U} / K)$. The category $\operatorname{Isoc}^{\dagger \dagger}(Y, \mathcal{P} / K)$ only depends on $\mathbb{Y}$ and $K$ and can be denoted by $\operatorname{Isoc}^{\dagger \dagger}(\mathbb{Y} / K)$. Moreover, we denote by $D_{\text {isoc }}^{\mathrm{b}}(Y, \mathcal{P} / K)$ the full subcategory of $D_{\text {ovhol }}^{\mathrm{b}}(Y, \mathcal{P} / K)$ consisting of complexes $\mathcal{E}$ such that $\mathcal{H}_{\mathrm{t}}^{j}(\mathcal{E}) \in \operatorname{Isoc}^{\dagger \dagger}(Y, \mathcal{P} / K)$ for any integer $j$. The category $D_{\text {isoc }}^{\mathrm{b}}(Y, \mathcal{P} / K)$ depends only on $\mathbb{Y}$ and $K$, and can be denoted by $D_{\text {isoc }}^{\mathrm{b}}(\mathbb{Y} / K)$.

(i) We have the equivalence of categories:

$$
F-\operatorname{Isoc}^{\dagger}(\mathbb{Y} / K) \cong F-\operatorname{Isoc}^{\dagger \dagger}(\mathbb{Y} / K)
$$

Indeed, by gluing lemma 1.2.12 and the gluing for overconvergent isocrystals, we can reduce to the case where $Z$ is the intersection of a divisor on $P$ with $X$, in which case we have already recalled above.

(ii) Let $E \in F$-Isoc ${ }^{\dagger}(\mathbb{Y} / K), y$ be a closed point of $Y$. Let $d$ be the dimension of $Y$ locally around $y$. By using $[$ Abe13, 5.6], we have the isomorphisms

$$
i_{y}^{+}\left(\operatorname{sp}_{+}(E)\right) \stackrel{\sim}{\rightarrow} i_{y}^{!}\left(\operatorname{sp}_{+}(E)\right)(d)[2 d] \stackrel{\sim}{\rightarrow} \operatorname{sp}_{+}\left(i_{y}^{*}(E)\right)(d)[d],
$$

where $i_{y}:(\{y\},\{y\}) \rightarrow \mathbb{Y}$ denotes the canonical morphism.

\subsection{Properties of six functors for couples}

In this subsection, we prove some fundamental properties for six functors defined in the previous subsection.

Proposition 1.3.1. Let $\mathbb{Y}$ be a couple. Then the dual functor $\mathbb{D}_{\mathbb{Y}}$ is t-exact (cf. Definition 1.2.11).

Proof. Let $(Y, X, \mathcal{P}, \mathcal{Q})$ be an l.p. frame of $\mathbb{Y}$. By Remark 1.2.7 (ii), we reduce to the case where $Y=X$ and then from Berthelot-Kashiwara theorem (see [Ber02, 5.3.3]) to the case where $Y=X=P$. The lemma follows from the exactness of $\mathbb{D}_{\mathcal{P}}$ (see [Vir00]).

Proposition 1.3.2. Let $u: \mathbb{Y}=(Y, X) \rightarrow \mathbb{Y}^{\prime}=\left(Y^{\prime}, X^{\prime}\right)$ be a morphism of couples.

(i) If $u$ is c-smooth of relative dimension $d$ such that the fibers are equidimensional, then $u^{+}[d]$ and $u^{!}[-d]$ are $t$-exact.

(ii) If $u$ is a c-immersion, then $u^{!}$(resp. $u^{+}$) is left t-exact (resp. right t-exact). Moreover, we have the canonical isomorphism $u^{!} \circ u_{+} \stackrel{\sim}{\rightarrow} i d$. 
(iii) (Kashiwara's theorem) Suppose $u$ is a c-closed immersion. Then $u_{+}$is t-exact. Moreover, $u_{+}$ (resp. $u_{+}^{0}$ ) is fully faithful. The objects of the essential image of $u_{+}$(resp. $u_{+}^{0}$ ) are called "with support in $\mathbb{Y} "$. Restricted to objects with support in $\mathbb{Y}$, the functor $u^{!}$is t-exact and $u^{!}$(resp. $u^{! 0}$ ) is canonically a quasi-inverse to $u_{+}$(resp. $u_{+}^{0}$ ).

Proof. Let us show (i). By Remark 1.2.7 (ii), we may assume that $u$ is of the form $u=(b, b):(Y, Y) \rightarrow$ $\left(Y^{\prime}, Y^{\prime}\right)$. The problem is local both on $Y$ and $Y^{\prime}$, so we may assume that there exists a factorization $Y \rightarrow Y^{\prime} \times \mathbb{A}^{d} \stackrel{p}{\rightarrow} Y^{\prime}$ where the first morphism is étale and the second is the projection. For the projection case, take a closed embedding $Y^{\prime} \hookrightarrow \mathcal{P}^{\prime}$, and let $\widetilde{p}: \mathcal{P}^{\prime} \times \widehat{\mathbb{A}}^{d} \rightarrow \mathcal{P}^{\prime}$. Then $p^{!}$is induced by $\widetilde{p}^{\prime}$, which is exact after shifting by the flatness of $\widetilde{p}$. Thus, we may assume that $b$ is étale. Take a closed point $y \in Y$. It suffices to show the exactness around $y$. By the structure theorem of étale morphism (cf. EGA IV, Theorem 18.4.6), locally around $y$ and $b(y)$, we can take the following cartesian diagram

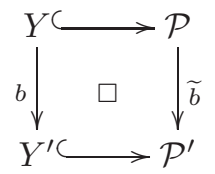

where $\mathcal{P}$ and $\mathcal{P}^{\prime}$ are smooth formal schemes, and $\widetilde{b}$ is étale. By the flatness of $\widetilde{b}$, the exactness follows.

Let us show (ii) and (iii). By duality, we may concentrate on showing the $u^{!}$case. By using Lemma 1.2.8, we may assume that $u$ is of the form $(b, \mathrm{id}):(Y, X) \rightarrow\left(Y^{\prime}, X\right)$, which reduce easily to already known cases (e.g. see Kashiwara's theorem proven by Berthelot in [Ber02]).

Proposition 1.3.3. (i) Using the notation of paragraph 1.1.8 (i), we have a canonical isomorphism (-) $\bigotimes_{K}$ $(-) \cong p^{+}(-) \otimes_{\mathbb{Y}^{\prime \prime}} p^{\prime+}(-)$.

(ii) Exterior tensor products are t-exact.

Proof. Let us show (i). Take l.p. frames $(Y, X, \mathcal{P}, \mathcal{Q})$ and $\left(Y^{\prime}, X^{\prime}, \mathcal{P}^{\prime}, \mathcal{Q}^{\prime}\right)$. Let $\widetilde{p}^{(\prime)}: \mathcal{P} \times \mathcal{P}^{\prime} \rightarrow \mathcal{P}^{(\prime)}$. Let $\mathcal{E}^{(\prime)} \in D_{\text {ovhol }}^{\mathrm{b}}\left(\mathcal{D}_{\mathcal{P}^{(\prime)}, \mathbb{Q}}^{\dagger}\right)$. Consider the homomorphism

$$
\mathbb{R} \underline{\Gamma}_{Y \times Y^{\prime}}^{\dagger}\left(\mathcal{E} \otimes_{K} \mathcal{E}^{\prime}\right) \rightarrow \mathcal{E} \otimes_{K} \mathcal{E}^{\prime}
$$

When $\mathcal{E}^{(\prime)} \in D_{\text {ovhol }}^{\mathrm{b}}\left(Y^{(\prime)}, X / K\right)$, this is an isomorphism. Indeed, the right side is supported on $\overline{Y \times Y^{\prime}}$, the closure of $Y \times Y^{\prime}$ in $P \times P^{\prime}$. Putting $Z:=\overline{Y \times Y^{\prime}} \backslash Y \times Y^{\prime}$, it remains to show that $\left({ }^{\dagger} Z\right)\left(\mathcal{E} \otimes \mathcal{E}^{\prime}\right)=0$. This follows by (1.1.9.1). Thus the proposition is reduced to showing that there exists an isomorphism $\mathbb{D}_{\mathcal{P} \times \mathcal{P}^{\prime}}\left(\mathcal{E} \otimes \mathcal{E}^{\prime}\right) \cong \mathbb{D}_{\mathcal{P}}(\mathcal{E}) \otimes \mathbb{D}_{\mathcal{P}^{\prime}}\left(\mathcal{E}^{\prime}\right)$ for $\mathcal{E}^{(\prime)} \in D_{\text {perf }}\left(\mathcal{D}_{\mathcal{P}^{(\prime)}, \mathbb{Q}}^{\dagger}\right)$. Since $\widehat{\mathcal{D}}_{\mathcal{P}}^{(m)}$ is of finite cohomological dimension, by [Vir00, I.4], it suffices to show that $\mathbb{D}_{\mathcal{P} \times \mathcal{P}^{\prime}}^{(m)}\left(\mathcal{E}^{(m)} \otimes \mathcal{E}^{\prime(m)}\right) \cong \mathbb{D}_{\mathcal{P}}^{(m)}\left(\mathcal{E}^{(m)}\right) \otimes \mathbb{D}_{\mathcal{P}^{\prime}}^{(m)}\left(\mathcal{E}^{(m)}\right)$ for $\mathcal{E}^{(\prime)(m)} \in$ $D_{\text {perf }}\left(\widehat{\mathcal{D}}_{\mathcal{P}\left({ }^{\prime}\right)}^{(m)}\right)$. By passing to the limit, we are reduced to the following fact, whose verification is easy: let $\mathcal{R}$ be a commutative ring on a topos, and $\mathcal{A}, \mathcal{B}$ be flat $R$-algebras such that $\mathcal{R}$ is the center of them. Let $\mathcal{M} \in D_{\text {perf }}^{\mathrm{b}}(\mathcal{A}), \mathcal{N} \in D_{\text {perf }}^{\mathrm{b}}(\mathcal{B})$. Putting $\mathcal{C}:=\mathcal{A} \otimes_{\mathcal{R}} \mathcal{B}$, which is a $\mathcal{R}$-algebra, we have an isomorphism

$$
\mathbb{R} \mathcal{H} \operatorname{mom}_{\mathcal{A}}(\mathcal{M}, \mathcal{A}) \otimes_{\mathcal{R}}^{\mathbb{L}} \mathbb{R} \mathcal{H} \mathrm{m}_{\mathcal{B}}(\mathcal{N}, \mathcal{B}) \cong \mathbb{R} \mathcal{H} \mathrm{m}_{\mathcal{C}}\left(\mathcal{M} \otimes_{\mathcal{R}}^{\mathbb{L}} \mathcal{N}, \mathcal{C}\right) .
$$

To check the compatibility of Frobnius, recall the definition of the canonical isomorphism $F^{*} \circ \mathbb{D}^{(m)} \cong$ $\mathbb{D}^{(m+1)} \circ F^{*}$ defined in [Vir00, II.3.2]. Going back to this definition, the verification is straightforward.

Let us check (ii). For an immersion $u$ of couples, $u_{+}$and $\widetilde{\otimes}$ commute by (1.1.9.1). Thus, by dévissage, the lemma is reduced to the overconvergent $F$-isocrystal case. In this case, the verification is easy.

1.3.4. Let $u=(\star, a): \mathbb{Y}=(Y, X) \rightarrow\left(Y^{\prime}, X^{\prime}\right)=\mathbb{Y}^{\prime}$ be a complete morphism of couples. In this paragraph, we construct the canonical homomorphism $\theta_{u}: u_{!} \rightarrow u_{+}$.

Let $\mathcal{E} \in D_{\text {ovhol }}^{\mathrm{b}}(\mathbb{Y} / K)$. The morphism $u$ factors as $\mathbb{Y} \stackrel{\iota}{\rightarrow}(U, X)=\mathbb{U} \stackrel{u^{\prime}}{\rightarrow} \mathbb{Y}^{\prime}$, where $U:=a^{-1}\left(Y^{\prime}\right)$. Let $Z:=U \backslash Y$. Since $\mathbb{D}_{\mathbb{Y}}=\left({ }^{\dagger} Z\right) \circ \mathbb{D}_{\mathbb{U}}$ and since $Z$ is closed in $Y$, we get the canonical homomorphism 
$\mathbb{D}_{\mathbb{U}} \circ \mathbb{D}_{\mathbb{Y}}(\mathcal{E}) \rightarrow \mathbb{D}_{\mathbb{Y}} \circ \mathbb{D}_{\mathbb{Y}}(\mathcal{E}) \cong \mathcal{E}$, which yields the functorial homomorphism $\iota_{!}(\mathcal{E}) \rightarrow \iota_{+}(\mathcal{E})$. Moreover, since $u^{\prime}$ is c-proper, we have the relative duality isomorphism $u_{!}^{\prime} \stackrel{\sim}{\rightarrow} u_{+}^{\prime}$. We define

$$
\theta_{u}: u_{!} \cong u_{!}^{\prime} \circ \iota_{!} \rightarrow u_{!}^{\prime} \circ \iota_{+} \cong u_{+}^{\prime} \circ \iota_{+} \cong u_{+} .
$$

In order to check the transitivity in Proposition 1.3.7, we need the following lemmas.

Lemma 1.3.5. Let $(X, \mathcal{A})$ be a ringed space (where $\mathcal{A}$ is not necessary commutative), and $\mathcal{B} \rightarrow \mathcal{C}$ be a homomorphism in the derived category $D^{\mathrm{b}}\left(\mathcal{A} \otimes_{\mathbb{Z}} \mathcal{A}\right)$. For any $\mathcal{A}$-complex $\mathcal{E}$, and $\star \in\{\mathcal{B}, \mathcal{C}\}$, we put $\mathbb{D}_{\star}(\mathcal{E}):=\mathbb{R H}_{\mathcal{H}} \mathrm{m}_{\mathcal{A}}(\mathcal{E}, \star)$, where we used the first $\mathcal{A}$-module structure of $\star$ to take $\mathcal{H o m}$, and consider $\mathbb{D}_{\star}(\mathcal{E})$ as a left $\mathcal{A}$-module using the second $\mathcal{A}$-module structure of $\star$. Let $\beta: \mathcal{E} \rightarrow \mathbb{D}_{\mathcal{B}} \circ \mathbb{D}_{\mathcal{B}}(\mathcal{E})$ be the canonical homomorphism. Then the following diagram is commutative:

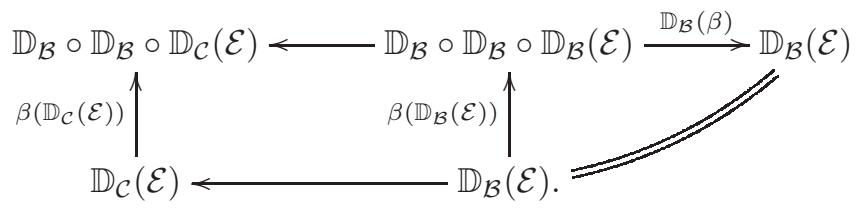

Proof. We only need to check the commutativity of the right triangle, whose verification is left to the reader.

Lemma 1.3.6. Consider the following left commutative diagram of l.p. frames:

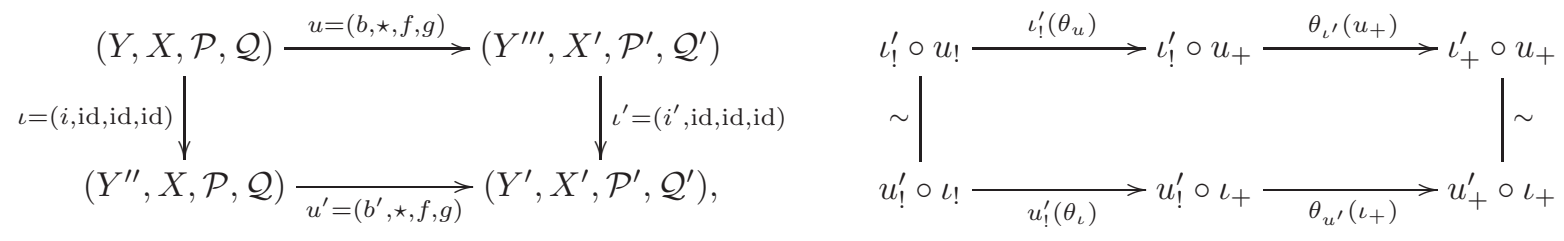

Assume that all the morphisms in the diagram are complete, and $b, b^{\prime}$ are proper. Then the right diagram above of functors from $(F-) D_{\mathrm{ovhol}}^{\mathrm{b}}(Y, \mathcal{P} / K)$ to $(F-) D_{\mathrm{ovhol}}^{\mathrm{b}}\left(Y^{\prime}, \mathcal{P}^{\prime} / K\right)$ is commutative.

Proof. Consider the following diagram, where we omit subscripts $\mathcal{P}$ and $\mathcal{P}^{\prime}$ from $\mathbb{D}$ :

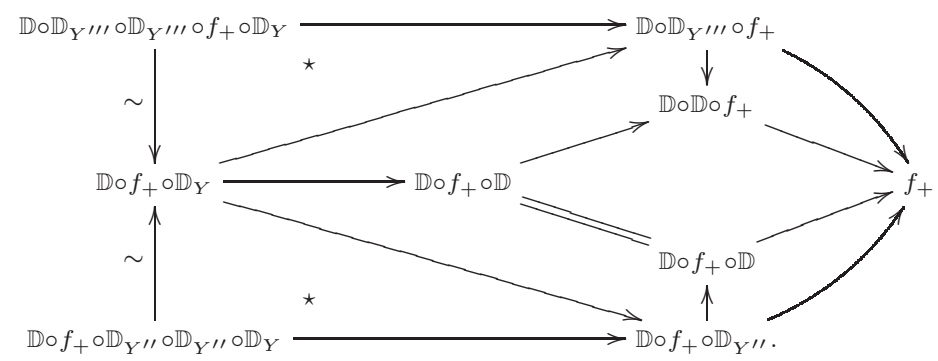

By taking the functor $\mathbb{R} \underline{\Gamma}_{Y^{\prime}}^{\dagger}$, we get the desired diagram. Thus, it suffices to check the commutativity of the big diagram above. The commutativity of small diagrams except for the one with $\star$ are easy. To check the commutativity of the triangle diagrams marked with $\star$, we need to check the commutativity of the following diagram:

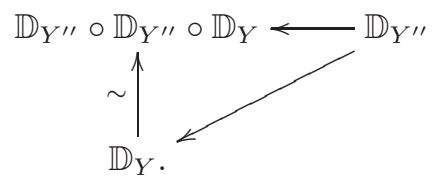


Take a set of divisors $\left\{T_{i}\right\}_{1 \leq i \leq r^{\prime \prime}}$ of $P$ such that $X \backslash\left(\bigcap_{\left.1 \leq i \leq r^{(\prime \prime}\right)} T_{i}\right)=Y^{(\prime \prime)}$ for some $r^{\prime \prime} \leq r$. Consider the following Čech type complexes

$$
\begin{aligned}
& \mathcal{D}^{(\prime \prime)}= \\
& \mathbf{s}\left[\begin{array}{cc}
\left.\mathcal{D}_{\mathcal{P}, \mathbb{Q}}^{\dagger} \rightarrow \bigoplus_{\left.1 \leq k \leq r^{(\prime \prime}\right)} \mathcal{D}_{\mathcal{P}, \mathbb{Q}}^{\dagger}\left({ }^{\dagger} T_{k}\right) \rightarrow \bigoplus_{\left.1 \leq k_{1}<k_{2} \leq r^{(\prime \prime}\right)} \mathcal{D}_{\mathcal{P}, \mathbb{Q}}^{\dagger}\left({ }^{\dagger} T_{k_{1}} \cup T_{k_{2}}\right) \rightarrow \ldots \rightarrow \mathcal{D}_{\mathcal{P}, \mathbb{Q}}^{\dagger}{ }^{\dagger} T_{1} \cup \cdots \cup T_{\left.r^{(\prime \prime}\right)}\right) \\
\downarrow & \downarrow \\
\mathcal{D}_{\mathcal{P}, \mathbb{Q}}^{\dagger} \longrightarrow & \downarrow
\end{array}\right]
\end{aligned}
$$

where $\mathcal{D}_{\mathcal{P}, \mathbb{Q}}^{\dagger}$ of the first line is placed at degree $(0,0)$, and $\mathbf{s}$ denotes the simple complex associated with the double complex. We have the canonical homomorphism of complexes $\mathcal{D} \rightarrow \mathcal{D}^{\prime \prime}$. Recall that

$$
\mathbb{D}_{Y^{(\prime \prime)}}(-) \cong \mathbb{R} \mathcal{H} \mathrm{om}_{\mathcal{D}_{\mathcal{P}, \mathbb{Q}}^{\dagger}}\left(-, \mathcal{D}^{(\prime \prime)}\right) \otimes_{\mathcal{O}_{\mathcal{P}}} \omega_{\mathcal{P}}^{-1}\left[d_{P}\right]
$$

The commutativity follows from a lemma on general non-sense Lemma 1.3.5 above.

Proposition 1.3.7. Let $u: \mathbb{Y} \rightarrow \mathbb{Y}^{\prime}, u^{\prime}: \mathbb{Y}^{\prime} \rightarrow \mathbb{Y}^{\prime \prime}$ be two complete morphisms of couples. We have a canonical isomorphism $\theta_{u^{\prime}}\left(u_{+}\right) \circ u_{!}^{\prime}\left(\theta_{u}\right) \cong \theta_{u^{\prime} \circ u}$.

Proof. This follows from Lemma 1.3.6.

Lemma 1.3.8. Let $u: \mathbb{Y} \rightarrow \mathbb{Y}^{\prime}$ be a complete morphism of couples. Then the following diagram of functors from $F$ - $D_{\mathrm{ovhol}}^{\mathrm{b}}(\mathbb{Y} / K)$ to $F-D_{\mathrm{ovhol}}^{\mathrm{b}}\left(\mathbb{Y}^{\prime} / K\right)$ is commutative.

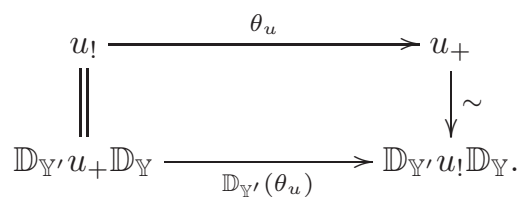

Proof. Let $u=(\star, a)$, and consider the factorization $\mathbb{Y}=(Y, X) \stackrel{i}{\rightarrow} \mathbb{Y}^{\prime \prime}:=\left(a^{-1}\left(Y^{\prime}\right), X\right) \stackrel{p}{\rightarrow} \mathbb{Y}^{\prime}=\left(Y^{\prime}, X^{\prime}\right)$. Let $\mathcal{E} \in F$ - $D_{\text {ovhol }}^{\mathrm{b}}(\mathbb{Y} / K)$. Consider the following diagram:

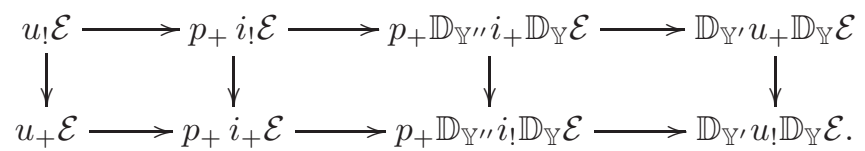

Since the outer two squares are commutative, it suffices to treat the $u=i$ case, and to show that the diagram

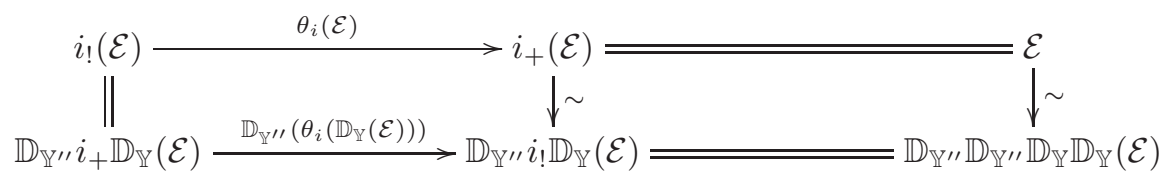

is commutative. We assume $u=i$, namely $X=X^{\prime}$ and $Y \subset Y^{\prime} \subset X$, in the following. Take an l.p. frame $\left(Y^{\prime}, X, \mathcal{P}, \mathcal{Q}\right)$, and put $T:=X \backslash Y$. We denote $\mathbb{D}_{Y^{(\prime)}, \mathcal{P}}$ by $\mathbb{D}_{Y^{(\prime)}}$. The homomorphism $\alpha$ is the one induced by id $\rightarrow\left({ }^{\dagger} T\right)$ and $\beta$ is the biduality isomorphisms id $\stackrel{\sim}{\rightarrow} \mathbb{D}_{Y} \mathbb{D}_{Y}=\left({ }^{\dagger} T\right) \mathbb{D}_{Y^{\prime}} \mathbb{D}_{Y}$. Let us consider the following diagram of $F-\mathcal{D}_{\mathcal{P}, \mathbb{Q}^{-c o m p l e x e s: ~}}^{\dagger}$

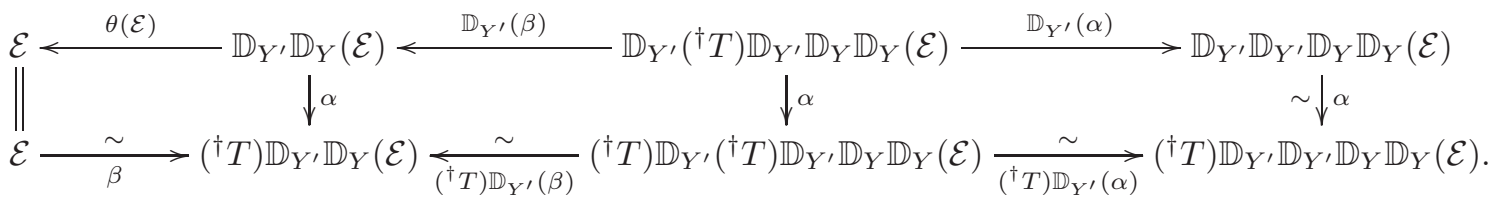


This diagram is commutative by definition and by functoriality. Composing the morphisms of the bottom and next the right vertical morphism (i.e. $\alpha^{-1}$ ) of $(\star \star)$, we get an isomorphism $\gamma: \mathcal{E} \stackrel{\sim}{\rightarrow} \mathbb{D}_{Y}, \mathbb{D}_{Y}, \mathbb{D}_{Y} \mathbb{D}_{Y}(\mathcal{E})$. Since the diagram $(\star \star)$ is commutative, it is sufficient to check that this isomorphism $\gamma$ is equal to the right arrow of $(\star)$. Let us consider the following diagram:

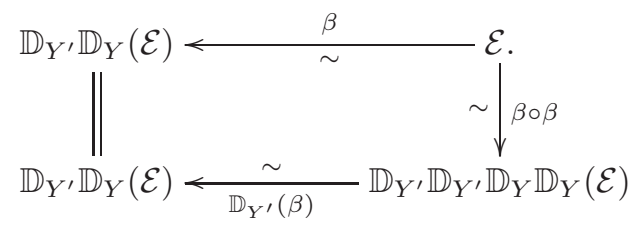

We remark that, in this case, the right vertical morphism of $(\star \star \star)$ is the right morphism of $(\star)$ and that $\gamma=\mathbb{D}_{\mathcal{P}}(\beta) \circ \beta^{-1}$. Thus, it remains to check the commutativity of $(\star \star \star)$. For this, we argue as in the last part of the proof of Lemma 1.3.6.

Lemma 1.3.9. Consider the following cartesian diagram $D$ on the left of l.p. frames (i.e. the four underlying squares are cartesian):
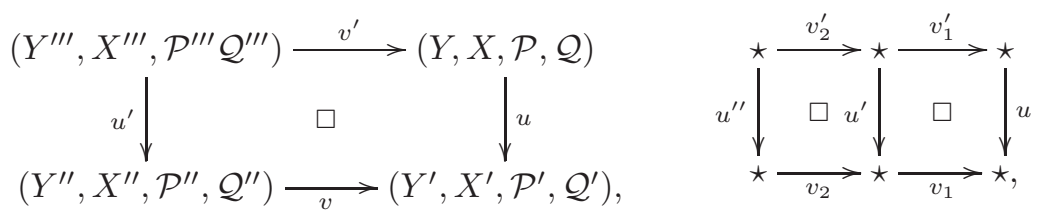

such that $u$ is complete (which implies the completeness of $u^{\prime}$ as well). Then we have a canonical base change isomorphism $b_{D}: v^{!} u_{+} \stackrel{\sim}{\rightarrow} u_{+}^{\prime} v^{\prime !}$. This base change isomorphism is compatible with compositions with respect to $v$ in the following sense: consider the diagram on the right above. The stars denote some l.p. frames, and $u$ is assumed to be complete. Its right (resp. left) square is called $D$ (resp. D') and the outer big diagram is called E. Then the composition

$$
\left(v_{1} \circ v_{2}\right)^{!} \circ u_{+} \stackrel{b_{D}}{\sim} v_{2}^{!} \circ u_{+}^{\prime} \circ v_{1}^{\prime !} \stackrel{b_{D^{\prime}}}{\sim} u_{+}^{\prime} \circ\left(v_{1}^{\prime} \circ v_{2}^{\prime}\right)^{!}
$$

is equal to $b_{E}$. Similarly the base change isomorphism is compatible with composition with respect to $u$ as well.

Proof. Let $v:(Y, X, \mathcal{P}, \mathcal{Q}) \rightarrow\left(Y^{\prime}, X^{\prime}, \mathcal{P}^{\prime}, \mathcal{Q}^{\prime}\right)$ be a morphism of l.p. frames. The morphism $v$ is said to be cartesian if $\mathcal{P}=\mathcal{Q} \times{ }_{\mathcal{Q}^{\prime}} \mathcal{P}^{\prime}, X=Q \times{ }_{Q^{\prime}} X^{\prime}, Y=Q \times{ }_{Q^{\prime}} Y^{\prime}$, of type $I$ if $g$ is an immersion, and of type $I I$ if it is cartesian and $g$ is smooth. We notice that $v$ factors canonically as $(Y, X, \mathcal{P}, \mathcal{Q}) \stackrel{\iota}{\rightarrow}\left(Q \times Y^{\prime}, Q \times X^{\prime}, \mathcal{Q} \times\right.$ $\left.\mathcal{P}^{\prime}, \mathcal{Q} \times \mathcal{Q}^{\prime}\right) \stackrel{v^{\prime}}{\rightarrow}\left(Y^{\prime}, X^{\prime}, \mathcal{P}^{\prime}, \mathcal{Q}^{\prime}\right)$ where $\iota$ is of type I and $v^{\prime}$ is II. We can define the base change isomorphism for morphisms of type I and II individually. Indeed, for type I, the base change isomorphism is nothing but the transitivity of the local cohomology functor and for type II, this is [Abe13, 10.7]. These isomorphisms are compatible with the composition of $u$. The base change isomorphism for $v$ is defined to be the composition.

It remains to show that the isomorphism is compatible with composition with respect to $v$. To construct such a natural transform, we may reduce to the following commutative and cartesian diagrams
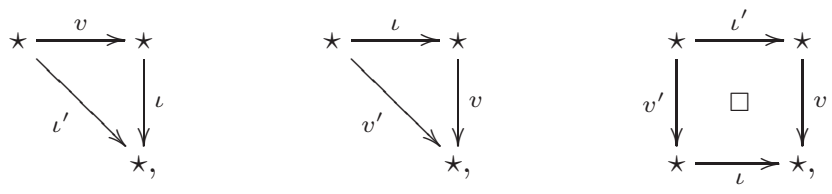

where $\iota$ and $\iota^{\prime}$ are morphisms of type I and $v$ and $v^{\prime}$ are of type II, and construct a natural isomorphism between the composition of the base change isomorphism of $\iota, v^{\prime}$ and $\iota^{\prime}$, $v$. The first one easily follows 
using the equivalence in Lemma 1.2.8, and the last one is similar to this. Let us check the second one. Let $v^{\prime}:\left(Y^{\prime}, X^{\prime}, \mathcal{P}^{\prime}, \mathcal{Q}^{\prime}\right) \rightarrow(Y, X, \mathcal{P}, \mathcal{Q})$ and $v:\left(Y^{\prime \prime}, X^{\prime \prime}, \mathcal{P}^{\prime \prime}, \mathcal{Q}^{\prime \prime}\right) \rightarrow(Y, X, \mathcal{P}, \mathcal{Q})$. Let $i$ be the immersion $\mathcal{Q}^{\prime} \hookrightarrow \mathcal{Q}^{\prime \prime}$ defining $\iota$, and $\widetilde{v}: \mathcal{Q}^{\prime} \rightarrow \mathcal{Q}$ be the one defining $v$. Since $v$ and $v^{\prime}$ are assumed to be cartesian, $\iota$ is cartesian as well, and $\iota^{!}$is nothing but $i^{!}$. Thus the base change theorem is reduced to showing the following claim, whose verification is left to the reader.

Claim. Keeping the notation, consider the following cartesian diagram of smooth formal schemes:

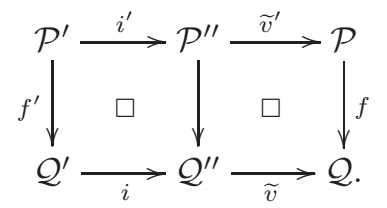

Then the two canonical base change isomorphisms between functors

$$
(\widetilde{v} \circ i)^{!} \circ f_{+} \cong f_{+}^{\prime} \circ\left(\widetilde{v}^{\prime} \circ i^{\prime}\right)^{!}: D_{\mathrm{coh}}^{\mathrm{b}}\left(\mathcal{D}_{\mathcal{P}, \mathbb{Q}}^{\dagger}\right) \rightarrow D^{\mathrm{b}}\left(\mathcal{D}_{\mathcal{Q}^{\prime}, \mathbb{Q}}^{\dagger}\right)
$$

are identical.

Proposition 1.3.10 (Base change). Consider the following diagram of couples which induces an isomorphism $Y^{\prime \prime \prime} \cong Y \times_{Y^{\prime}} Y^{\prime \prime}$ :

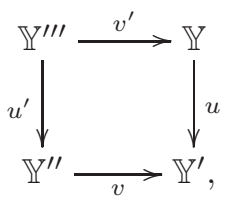

where $u$ and $u^{\prime}$ are complete. Then we have a canonical isomorphism $v^{!} \circ u_{+} \cong u_{+}^{\prime} \circ v^{\prime !}$. This isomorphism is compatible with composition with respect to both $u$ and $v$.

Proof. Let $\mathbb{Y}^{*}=\left(Y^{*}, X^{*}\right)$ where $* \in\{\emptyset, \prime, \prime \prime, \prime \prime \prime\}$. The diagram can be supplemented as follows:

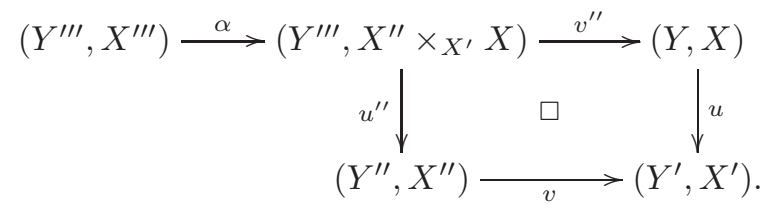

Let us construct the base change isomorphism for cartesian diagram of couples: $v^{!} \circ u_{+} \cong u_{+}^{\prime \prime} \circ v^{\prime \prime !}$. To construct this isomorphism, take a following cartesian diagram

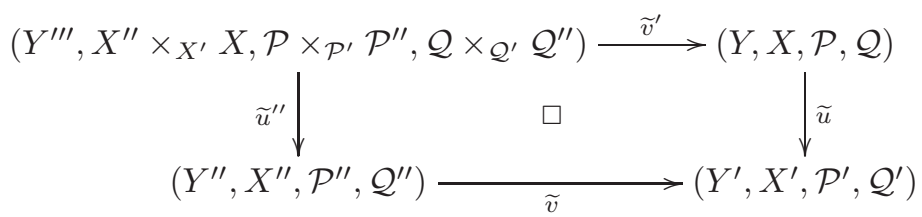

where $\widetilde{u}$ and $\widetilde{v}$ are morphisms of frames of $u$ and $v$ respectively such that the morphism of formal schemes are smooth. Then applying Lemma 1.3.9, we have a base change isomorphism $\widetilde{v}^{!} \circ \widetilde{u}_{+} \cong \widetilde{u}_{+}^{\prime \prime} \circ \widetilde{v}^{\prime \prime !}$. We need to check that the isomorphism does not depend on the choice of $\widetilde{u}$ and $\widetilde{v}$. This follows easily from the compatibility of the composition.

Now, we define the desired base change isomorphism to be $v^{!} \circ u_{+} \cong u_{+}^{\prime \prime} \circ v^{\prime \prime !} \cong u_{+}^{\prime \prime} \circ\left(\alpha+\circ \alpha^{!}\right) \circ v^{\prime \prime !} \cong u_{+}^{\prime} \circ v^{\prime !}$. To check the compatibility, we need diagram chasing using Lemma 1.3.9, which is tedious but not difficult. 
Lemma 1.3.11. Let $\mathbb{Y}$ be a couple, and $\mathcal{E} \in F-D_{\mathrm{ovhol}}^{\mathrm{b}}(\mathbb{Y} / K)$. Assume, for any closed point $y$ of $Y$, we have $i_{y}^{!}(\mathcal{E})=0$, where $i_{y}:(\{y\},\{y\}) \rightarrow \mathbb{Y}$ is the canonical morphism. Then $\mathcal{E}=0$.

Proof. From Lemma 1.2.3, one can suppose $\mathbb{Y}$ is of the form $(Y, Y)$. We proceed by induction on the dimension of the support of $\mathcal{E}$. We may assume that the support of $\mathcal{E}$ is $Y$. There exists a closed subscheme $Z \subset Y$ such that $U:=Y \backslash Z$ is dense and smooth, and $j^{!} \mathcal{E}$ is in $F$ - $D_{\text {isoc }}^{\mathrm{b}}(U, Y / K)$ where $j:(U, Y) \rightarrow(Y, Y)$ (cf. paragraph 1.2.14). Since for $u \in U$ we have $i_{u}^{\prime !} j^{!} \mathcal{E}=0$ where $i_{u}:(\{u\},\{u\}) \rightarrow(U, Y)$, we have $j ! \mathcal{E}=0$. Consider the localization triangle $\mathbb{R} \underline{\Gamma}_{Z}^{\dagger}(\mathcal{E}) \rightarrow \mathcal{E} \rightarrow\left({ }^{\dagger} Z\right)(\mathcal{E}) \stackrel{+}{\rightarrow}$. Since for any $y \in Z, i_{y}^{!}\left(\mathbb{R} \underline{\Gamma}_{Z}^{\dagger} \mathcal{E}\right)=0$, we have $\mathbb{R} \underline{\Gamma}_{Z}^{\dagger}(\mathcal{E})=0$ by induction hypothesis. We conclude since $\left({ }^{\dagger} Z\right)(\mathcal{E})=j_{+} j^{!} \mathcal{E}=0$.

Proposition 1.3.12. Let $u: \mathbb{Y}^{\prime} \rightarrow \mathbb{Y}$ be a morphism of couples. If $u$ is a c-universal homeomorphism (i.e. by EGA IV, Proposition 8.11.6, finite, surjective and radicial), then the functors $u_{+}$and $u^{!}$induce canonical equivalence of categories between $F-D_{\text {ovhol }}^{\mathrm{b}}(\mathbb{Y} / K)$ and $F-D_{\text {ovhol }}^{\mathrm{b}}\left(\mathbb{Y}^{\prime} / K\right)$.

Proof. Let $\mathcal{E} \in F-D_{\text {ovhol }}^{\mathrm{b}}(\mathbb{Y} / K)$ and $\mathcal{E}^{\prime} \in F-D_{\text {ovhol }}^{\mathrm{b}}\left(\mathbb{Y}^{\prime} / K\right)$. It is sufficient to check that adjunction morphisms $u_{+} \circ u^{!}(\mathcal{E}) \rightarrow \mathcal{E}$ and $\mathcal{E}^{\prime} \rightarrow u^{!} \circ u_{+}\left(\mathcal{E}^{\prime}\right)$ are isomorphisms. Let $y$ be a closed point $Y$ and $y^{\prime}:=u^{-1}(y)$. We denote by $i_{y}: y \hookrightarrow Y$ (resp. $i_{y^{\prime}}: y^{\prime} \hookrightarrow Y^{\prime}$ ) the canonical closed immersion, and by $u_{y}: y^{\prime} \rightarrow y$ the induced morphism, which is in fact an isomorphism since $u$ is surjective, radicial, and $k$ is perfect. From the base change theorem 1.3.10, we get $i_{y}^{!} \circ u_{+} \stackrel{\sim}{\rightarrow} u_{y+} \circ i_{y^{\prime}}^{!}$. By applying $i_{y}^{!}$to the canonical morphism $u_{+} \circ u^{!}(\mathcal{E}) \rightarrow \mathcal{E}$ we get the adjunction morphism $u_{y+} \circ u_{y}^{!}\left(i_{y}^{!} \mathcal{E}\right) \rightarrow i_{y}^{!} \mathcal{E}$, which is an isomorphism. From Lemma 1.3.11, this implies that the morphism $u_{+} \circ u^{!}(\mathcal{E}) \rightarrow \mathcal{E}$ is an isomorphism. We may check similarly that $\mathcal{E}^{\prime} \rightarrow u^{!} \circ u_{+}\left(\mathcal{E}^{\prime}\right)$ is an isomorphism as well.

Proposition 1.3.13. Let $u: \mathbb{Y} \rightarrow \mathbb{Y}^{\prime}$ be a complete morphism of couples.

(i) If $u$ is c-affine, then $u_{+}$(resp. $\left.u_{!}\right)$is right t-exact (resp. left t-exact).

(ii) If $u$ is c-quasi-finite, then $u_{+}$(resp. $\left.u_{!}\right)$is left t-exact (resp. right t-exact).

Proof. Let us show (i). Take a morphism of l.p. frames $(\star, \star, \star, g):(Y, X, \mathcal{P}, \mathcal{Q}) \rightarrow\left(Y^{\prime}, X^{\prime}, \mathcal{P}^{\prime}, \mathcal{Q}^{\prime}\right)$ of $u$. From Remark 1.2.7, we may assume $Y^{\prime}=P^{\prime}$. Since the claim is local, we may assume $\mathcal{P}^{\prime}$ to be affine. Since $Y$ is affine, we can take a closed immersion $Y \hookrightarrow \widehat{\mathbb{A}}_{\mathcal{P}^{\prime}}^{n}$ for some $n$. Since $g$ is assumed to be smooth, the canonical morphism $\widehat{\mathbb{P}}_{\mathcal{P}^{\prime}}^{n} \times \times_{\mathcal{P}^{\prime}} \mathcal{P} \rightarrow \mathcal{P}^{\prime}$ is proper smooth, and we may consider the following commutative diagram of frames:

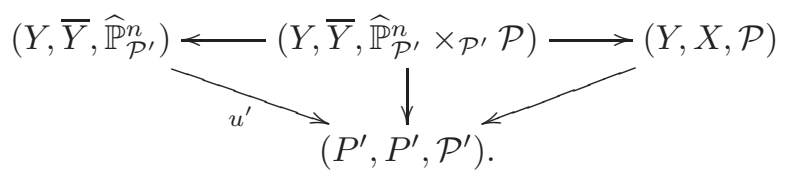

Thus, we are reduced to showing the right exactness of $u_{+}^{\prime}$. Let $D \subset \widehat{\mathbb{P}}_{\mathcal{P}^{\prime}}^{n}$ be the divisor at infinity. By construction, $Y \hookrightarrow \widehat{\mathbb{P}}_{\mathcal{P}^{\prime}}^{n} \backslash D=\widehat{\mathbb{A}}_{\mathcal{P}^{\prime}}^{n}$ is a closed immersion, and we may assume that $Y=\mathbb{A}_{P^{\prime}}^{n}$ by Proposition 1.3.2 (iii). Then the proposition follows by [NH97, 5.4.1].

Let us show (ii). By exactness of the dual functor, it suffices to show the $u_{+}$case. First, consider the case where $u$ is a c-open immersion. By Lemma 1.2.8, we can suppose $X=X^{\prime}$. Then, the claim follows from Remark 1.2.7 (iv). The case where $u$ is a c-closed immersion is treated in Proposition 1.3.2. Finally, let us show the general case by the induction on the dimension of $X$. We may assume that the image of $b$ is dense in $Y^{\prime}$. By using the localization triangle and the induction hypothesis, we may shrink $Y$, and assume that $Y$ is irreducible. For a morphism of frames $a:(\star, \star, \star$, id $):\left(Y_{\text {red }}, \star, \star, \mathcal{Q}\right) \rightarrow(Y, \star, \star, \mathcal{Q}), a_{+}$is t-exact by definition. Thus we may replace $Y$ and $Y^{\prime}$ by $Y_{\text {red }}$ and $Y_{\text {red }}^{\prime}$, and assume that $Y$ and $Y^{\prime}$ are integral. By using Zariski main theorem, there exists an open dense subscheme $U^{\prime} \subset Y^{\prime}$ such that $u^{-1}\left(U^{\prime}\right) \rightarrow U^{\prime}$ is finite étale. Using the proven immersion case and the induction hypothesis, we may shrink $Y^{\prime}$, and reduced to showing the case where $Y^{\prime}$ is smooth and $u$ is c-finite étale. In this case, we see easily that $u_{+}$is t-exact.

1.3.14. Let $Y$ be a realizable variety. Take a couple $(Y, X)$ such that $X$ is proper. Then we define the category $(F-) D_{\text {ovhol }}^{\mathrm{b}}(Y / K):=(F-) D_{\text {ovhol }}^{\mathrm{b}}(Y, X / K)$. This does not depend on the choice of such a couple 
up to canonical equivalence of categories with t-structure. Indeed, take another couple $\left(Y, X^{\prime}\right)$ with $X^{\prime}$ is proper. Let $X^{\prime \prime}$ be the closure of $Y$ in $X \times X^{\prime}$. We obtain two morphisms $\left(Y, X^{\prime \prime}\right) \rightarrow\left(Y, X^{(\prime)}\right)$. Then, using Lemma 1.2.8 the independence follows.

With these categories with Frobenius structures, we get six functors formalism on the category of realizable varieties (without Frobenius structures, the definition of tensor products is problematic so far). Let $f: Y \rightarrow Y^{\prime}$ be a morphism of realizable varieties. We have the following properties:

(i). Dual functor on $Y$, denoted by $\mathbb{D}_{Y}$, is defined thanks to Lemma 1.2.8.3.

(ii). Tensor functor on $Y$, denoted by $\widetilde{\otimes}_{Y}$ or simply $\widetilde{\otimes}$.

(iii). Push-forward for $f$, denoted by $f_{+}$is defined by the transitivity of the push-forward for couples.

(iv). Extraordinary pull-back for $f$, denoted by $f^{!}$is defined by the transitivity as well.

(v). Extraordinary push-forward and ordinary pull-back for $f$, denoted by $f_{\text {! }}$ and $f^{+}$, is defined by (i), (iii), (iv).

(vi). We have a canonical homomorphism $f_{!} \rightarrow f_{+}$and this is an isomorphism if $f$ is proper (see paragraph 1.3.4).

(vii). We have a base change isomorphism by Proposition 1.3.10.

(viii). We have adjoint pairs $\left(f^{+}, f_{+}\right)$and $\left(f_{!}, f^{!}\right)$by Lemma 1.1.10.

\subsection{Intermediate extensions}

In this subsection, we define the intermediate extension functor, which plays an essential role in defining the intersection cohomology. Unless otherwise stated, we let $u=(b, a): \mathbb{Y}=(Y, X) \rightarrow \mathbb{Y}^{\prime}=\left(Y^{\prime}, X^{\prime}\right)$ be a c-immersion of couples in this subsection.

Definition 1.4.1. For $\mathcal{E} \in F$-Ovhol $(\mathbb{Y} / K)$, we have the homomorphism $\theta_{u, \mathcal{E}}^{0}:=\mathcal{H}_{\mathrm{t}}^{0}\left(\theta_{u, \mathcal{E}}\right): u_{!}^{0} \mathcal{E} \rightarrow u_{+}^{0} \mathcal{E}($ see Definition 1.2.9 and paragraph 1.3.4 for the notation). We define

$$
u !_{+}(\mathcal{E}):=\operatorname{Im}\left(\theta_{u, \mathcal{E}}^{0}: u_{!}^{0} \mathcal{E} \rightarrow u_{+}^{0} \mathcal{E}\right)
$$

This defines a functor $u_{!_{+}}: F$-Ovhol $(\mathbb{Y} / K) \rightarrow F$-Ovhol( $\left(\mathbb{Y}^{\prime} / K\right)$, and it is called the intermediate extension functor.

Remark 1.4.2. (i) It follows from Proposition 1.3 .13 that the functor $u_{!+}$preserves injections and surjections.

(ii) Since the functor $\mathbb{D}_{\mathbb{Y}^{\prime}}$ is t-exact on the category $F$-Ovhol $\left(\mathbb{Y}^{\prime} / K\right)$ by Proposition 1.3 .1 , for any $\mathcal{E} \in$ $F$-Ovhol $(\mathbb{Y} / K)$, we get the canonical isomorphisms:

$$
\begin{aligned}
\mathbb{D}_{\mathbb{Y}^{\prime}}\left(\operatorname{ker}\left(\theta_{u, \mathcal{E}}^{0}\right)\right) \stackrel{\sim}{\rightarrow} \operatorname{coker}\left(\mathbb{D}_{\mathbb{Y}^{\prime}}\left(\theta_{u, \mathcal{E}}^{0}\right)\right), \quad \mathbb{D}_{\mathbb{Y}^{\prime}}\left(\operatorname{coker}\left(\theta_{u, \mathcal{E}}^{0}\right)\right) \stackrel{\sim}{\rightarrow} \operatorname{ker}\left(\mathbb{D}_{\mathbb{Y}^{\prime}}\left(\theta_{\mathcal{E}}^{0}\right)\right), \\
\mathbb{D}_{\mathbb{Y}^{\prime}}\left(\operatorname{Im}\left(\theta_{u, \mathcal{E}}^{0}\right)\right) \stackrel{\sim}{\rightarrow} \operatorname{Im}\left(\mathbb{D}_{\mathbb{Y}^{\prime}}\left(\theta_{u, \mathcal{E}}^{0}\right)\right) .
\end{aligned}
$$

Moreover, when $u$ is c-affine, both functors $u_{+}, u_{!}: F$-Ovhol $(\mathbb{Y} / K) \rightarrow F$-Ovhol( $\left.\mathbb{Y}^{\prime} / K\right)$ are t-exact by Proposition 1.3.13, and we do not need to take $\mathcal{H}_{\mathrm{t}}^{0}$ in the definition of $u_{!+}$.

Corollary 1.4.3. Let $\mathcal{E} \in F$-Ovhol $(\mathbb{Y} / K)$. We have the canonical isomorphisms:

$$
u_{!+}(\mathcal{E}) \stackrel{\sim}{\rightarrow} \mathbb{D}_{\mathbb{Y}^{\prime}} \circ u_{!+} \circ \mathbb{D}_{\mathbb{Y}}(\mathcal{E}), \quad \operatorname{ker}\left(\theta_{u, \mathcal{E}}^{0}\right) \stackrel{\sim}{\rightarrow} \mathbb{D}_{\mathbb{Y}^{\prime}}\left(\operatorname{coker}\left(\theta_{u, \mathbb{D}_{\mathbb{Y}}(\mathcal{E})}^{0}\right)\right), \quad \operatorname{coker}\left(\theta_{u, \mathcal{E}}^{0}\right) \stackrel{\sim}{\rightarrow} \mathbb{D}_{\mathbb{Y}^{\prime}}\left(\operatorname{ker}\left(\theta_{u, \mathbb{D}_{\mathbb{Y}}(\mathcal{E})}^{0}\right)\right) .
$$

Proof. This is a consequence of Remark 1.4.2 (i) and Lemma 1.3.8. 
Remark 1.4.4. Let $\mathcal{E} \in F$-Ovhol $(\mathbb{Y} / K)$. Let $Y^{\prime \prime}$ be the closure of $Y$ in $Y^{\prime}$ and $Z:=Y^{\prime \prime} \backslash Y$. Since $\theta_{u, \mathcal{E}}^{0}$ is an isomorphism outside $Z$, this implies that the $F$-modules $\operatorname{ker}\left(\theta_{u, \mathcal{E}}^{0}\right)$ and $\operatorname{coker}\left(\theta_{u, \mathcal{E}}^{0}\right)$ have their support in $Z$ (cf. Proposition 1.3.2). Since $u_{+}$and $\mathbb{R} \underline{\Gamma}_{Z}^{\dagger}$ are left t-exact by Propositions 1.3.2 and 1.3.13, we get $0=\mathcal{H}_{\mathrm{t}}^{0}\left(\mathbb{R} \underline{\Gamma}_{Z}^{\dagger} \circ u_{+}(\mathcal{E})\right) \cong \mathbb{R}^{0} \underline{\Gamma}_{Z}^{\dagger}\left(u_{+}^{0} \mathcal{E}\right)$. Now applying the functor $\mathbb{R} \underline{\Gamma}_{Z}^{\dagger}$ to the exact sequence $0 \rightarrow u_{!_{+}}(\mathcal{E}) \rightarrow$ $u_{+}^{0}(\mathcal{E}) \rightarrow \operatorname{coker}\left(\theta_{u, \mathcal{E}}^{0}\right) \rightarrow 0$ (which can be viewed as an exact triangle) yields another short exact sequence:

$$
0 \rightarrow \operatorname{coker}\left(\theta_{u, \mathcal{E}}^{0}\right) \rightarrow \mathbb{R}^{1} \underline{\Gamma}_{Z}^{\dagger}\left(u_{!+}(\mathcal{E})\right) \rightarrow \mathbb{R}^{1} \underline{\Gamma}_{Z}^{\dagger}\left(u_{+}^{0}(\mathcal{E})\right) \rightarrow 0 .
$$

Suppose $u$ is affine (e.g. when $Z$ is a divisor of $Y^{\prime \prime}$ ). In this case, $\theta_{u, \mathcal{E}}=\theta_{u, \mathcal{E}}^{0}$ (i.e. $u_{+}$and $u$ ! are t-exact). Since $\mathbb{R} \underline{\Gamma}_{Z}^{\dagger}\left(u_{+}(\mathcal{E})\right)=0$, we get (in that case) the isomorphism:

$$
\operatorname{coker}\left(\theta_{u, \mathcal{E}}\right) \stackrel{\sim}{\rightarrow} \mathbb{R} \underline{\Gamma}_{Z}^{\dagger}\left(u !_{+}(\mathcal{E})\right)[1] .
$$

Lemma 1.4.5. Let $\mathbb{Y} \stackrel{u}{\rightarrow} \mathbb{Y}^{\prime} \stackrel{u^{\prime}}{\rightarrow} \mathbb{Y}^{\prime \prime}$ be c-immersions of couples.

(i) We have canonical equivalences of functors $u_{+}^{\prime 0} \circ u_{+}^{0} \cong\left(u^{\prime} \circ u\right)_{+}^{0}$, and $u_{!}^{\prime 0} \circ u_{!}^{0} \cong\left(u^{\prime} \circ u\right)_{!}^{0}$. This induces an equivalence $u_{!_{+}}^{\prime} \circ u_{!_{+}} \cong\left(u^{\prime} \circ u\right)_{!_{+}}$.

(ii) We have canonical isomorphisms $u^{\prime !} \circ\left(u^{\prime} \circ u\right)_{+}^{0} \stackrel{\sim}{\rightarrow} u_{+}^{0}, u^{\prime !} \circ\left(u^{\prime} \circ u\right)_{!}^{0} \stackrel{\sim}{\rightarrow} u_{!}^{0}$, and $u^{\prime} \circ\left(u^{\prime} \circ u\right)_{!_{+}} \stackrel{\sim}{\rightarrow} u_{!_{+}}$.

Proof. The first two isomorphisms follow by Proposition 1.3.13. Let $\mathcal{E} \in F$-Ovhol( $\mathbb{Y} / K)$. Using this, we have the canonical surjections: $u_{!}^{\prime 0} \circ u_{!}^{0}(\mathcal{E}) \rightarrow u_{!}^{\prime 0} \circ u_{!_{+}}(\mathcal{E}) \rightarrow u_{!_{+}}^{\prime} \circ u_{!_{+}}(\mathcal{E})$. We also have the canonical inclusions: $u_{!_{+}}^{\prime} \circ u_{!_{+}}(\mathcal{E}) \hookrightarrow u_{+}^{\prime 0} \circ u_{!_{+}}(\mathcal{E}) \hookrightarrow u_{+}^{\prime 0} \circ u_{+}^{0}(\mathcal{E})$. By functoriality, the composition of these homomorphisms is the canonical morphism $u_{!}^{\prime 0} \circ u_{!}^{0}(\mathcal{E}) \rightarrow u_{+}^{\prime 0} \circ u_{+}^{0}(\mathcal{E})$. Using the base change 1.3.10, the second part of the lemma is obvious.

Next proposition is a generalization of [Abe11, 2.1], and is generalized further at Theorem 1.4.9 below:

Lemma 1.4.6. Let $u: \mathbb{Y} \rightarrow \mathbb{Y}^{\prime}$ be a c-open immersion. Let $\mathcal{E}^{\prime}$ be an irreducible object of $F$-Ovhol $\left(\mathbb{Y}^{\prime} / K\right)$. Then $u^{! 0} \mathcal{E}^{\prime}$ is either irreducible in $F$-Ovhol $(\mathbb{Y} / K)$ or 0 .

Proof. Let $\alpha: u^{! 0} \mathcal{E}^{\prime} \rightarrow \mathcal{F}$ be a homomorphism with non-zero kernel. By adjointness 1.1.10 and $u^{!}=u^{+}$is t-exact, we get $\alpha^{\prime}: \mathcal{E}^{\prime} \rightarrow u_{+}^{0} \mathcal{F}$. Since $u^{!}\left(\operatorname{ker}\left(\alpha^{\prime}\right)\right) \cong \operatorname{ker}(\alpha) \neq 0$, we have $\operatorname{ker}\left(\alpha^{\prime}\right) \neq 0$. Since $\mathcal{E}^{\prime}$ is assumed to be irreducible, we have $\alpha^{\prime}=0$, and thus $\alpha=u^{! 0}\left(\alpha^{\prime}\right)=0$. Thus $u^{! 0} \mathcal{E}^{\prime}$ is irreducible or 0 .

Proposition 1.4.7. Let $\mathcal{E}$ be an irreducible object of $F-O v \operatorname{vhol}(\mathbb{Y} / K)$. Then:

(i) In the category $F-\mathrm{Ovhol}\left(\mathbb{Y}^{\prime} / K\right), u_{!_{+}}(\mathcal{E})$ is the unique irreducible subobject of $u_{+}^{0}(\mathcal{E})$.

(ii) In the category $F-O \operatorname{vhol}\left(\mathbb{Y}^{\prime} / K\right), u_{!_{+}}(\mathcal{E})$ is the unique irreducible quotient of $u_{!}^{0}(\mathcal{E})$.

(iii) Let $j: \mathbb{Y}^{\prime \prime} \rightarrow \mathbb{Y}^{\prime}$ be a c-open immersion. Then, in $F$-Ovhol $\left(\mathbb{Y}^{\prime \prime} / K\right)$, either $j^{!}\left(u_{+}^{0}(\mathcal{E})\right)=0$ or $j^{!}\left(u_{!_{+}}(\mathcal{E})\right)$ is the unique irreducible subobject of $j^{!}\left(u_{+}^{0}(\mathcal{E})\right)$.

Proof. The proof is essentially the same as that of $\left[\mathrm{BGK}^{+} 87\right.$, VII.10.5]. Let us show (i). By using BerthelotKashiwara theorem 1.3.2.(iii) and Lemma 1.4.5, we may assume that $u$ is a c-open immersion. We first claim that for any non-zero $F$-submodule $\mathcal{F}$ of $u_{+}^{0} \mathcal{E}, u^{!} \mathcal{F}$ is non-zero. Indeed, assume $u^{!} \mathcal{F}=0$, which is equivalent to assuming $\mathbb{R} \underline{\Gamma}_{Y}^{\dagger}(\mathcal{F})=0$ by Proposition 1.3.2.(ii). This means that $\mathcal{F}$ has its support in $Z(:=\bar{Y} \backslash Y)$ and then $\mathbb{R}^{0} \underline{\Gamma}_{Z}^{\dagger}(\mathcal{F}) \stackrel{\sim}{\rightarrow} \mathcal{F}$. This implies that $\mathcal{F} \subset \mathbb{R}^{0} \underline{\Gamma}_{Z}^{\dagger}\left(u_{+}^{0}(\mathcal{E})\right)$. By Remark 1.4.4, we have $\mathbb{R}^{0} \underline{\Gamma}_{Z}^{\dagger}\left(u_{+}^{0}(\mathcal{E})\right)=0$, thus $\mathcal{F}=0$, which contradicts with the assumption.

Let us return to the proof. Now, let $\mathcal{F}$ be an irreducible $F$-submodule of $u_{+}^{0} \mathcal{E}$. By the left t-exactness of $u^{!}$, we have $0 \neq u^{!} \mathcal{F} \subset u^{!} u_{+}^{0}(\mathcal{E}) \cong \mathcal{E}$. Since $\mathcal{E}$ is assumed to be irreducible, we have $u^{!} \mathcal{F}=\mathcal{E}$. Let $\mathcal{F}^{\prime}$ be another irreducible $F$-submodule of $u_{+}^{0} \mathcal{E}$ (resp. $\mathcal{F}^{\prime}:=u_{!+} \mathcal{E}$ ). Assume $\mathcal{F} \cap \mathcal{F}^{\prime}=0$. Left t-exactness of $u^{!}$ implies that $u^{!} \mathcal{F} \cap u^{!} \mathcal{F}^{\prime}=0$ in $\mathcal{E}$, which is impossible. Thus $\mathcal{F}=\mathcal{F}^{\prime}$ (resp. $\mathcal{F} \subset u_{!+} \mathcal{E}$ ), and $\mathcal{F}$ is the unique irreducible $F$-submodule of $u_{+}^{0} \mathcal{E}$. Let $\mathcal{Q}:=u_{!+}(\mathcal{E}) / \mathcal{F}$ be the quotient, and assume that this is not zero. This is supported in $Z$. We have

$$
\mathbb{D}_{\mathbb{Y}^{\prime}} \mathcal{Q} \subset \mathbb{D}_{\mathbb{Y}^{\prime}}\left(u_{!+} \mathcal{E}\right) \cong u_{!+}\left(\mathbb{D}_{\mathbb{Y}} \mathcal{E}\right) \subset u_{+}^{0}\left(\mathbb{D}_{\mathbb{Y}} \mathcal{E}\right)
$$


The claim above shows that $u^{!}\left(\mathbb{D}_{\mathbb{Y}^{\prime}} \mathcal{Q}\right)$ is non-zero, which is a contradiction with the fact that $\mathcal{Q}$ is supported in $Z$. Thus $\mathcal{Q}=0$, and $\mathcal{F}$ is equal to $u_{!+} \mathcal{E}$, which completes the proof of (i). By duality, (ii) follows.

To show (iii), use Lemma 1.4.6, and base change of couples of functors $\left(j^{!}, u_{+}\right)$and $\left(j^{!} \cong j^{+}, u_{+}\right)$. For details, we refer to $\left[\mathrm{BGK}^{+} 87\right]$.

We can extend Proposition 1.4.7 as follows:

Proposition 1.4.8. Let $\mathcal{E} \in F$-Ovhol $(\mathbb{Y} / K)$ and $\mathcal{E}^{\prime}$ be a subobject of $u_{+}(\mathcal{E})$ in $F$-Ovhol $\left(\mathbb{Y}^{\prime} / K\right)$. Assume that the inclusion $u^{!}\left(\mathcal{E}^{\prime}\right) \hookrightarrow u^{!} \circ u_{+}(\mathcal{E})=\mathcal{E}$ is an isomorphism. Then we have the canonical factorization $u_{!+}(\mathcal{E}) \subset \mathcal{E}^{\prime} \subset u_{+}(\mathcal{E})$ of the inclusion $u_{!_{+}}(\mathcal{E}) \subset u_{+}(\mathcal{E})$.

Proof. The proof is analogue to that of Proposition 1.4.7.(i).

Theorem 1.4.9. (i) Let $\mathcal{E}$ be an irreducible object of $F-\mathrm{Ovhol}(Y, X / K)$. Then there exists an open dense smooth subscheme $Y^{\prime}$ of $Y$ and $\mathcal{E}^{\prime} \in F-\operatorname{Isoc}^{\dagger \dagger}\left(Y^{\prime}, X / K\right)$ such that $\mathcal{E} \cong u !_{+}\left(\mathcal{E}^{\prime}\right)$ where $u:\left(Y^{\prime}, X\right) \rightarrow(Y, X)$ is the canonical c-inclusion.

(ii) Let $\mathbb{Y}$ be a couple, and $\left\{\mathbb{Y}_{i}\right\}_{i \in I}$ be an c-open covering of $\mathbb{Y}$ (cf. paragraph 1.2.12). Let $u_{i}: \mathbb{Y}_{i} \rightarrow \mathbb{Y}$ be the c-open immersions. Then $\mathcal{E}$ is semi-simple (resp. irreducible or 0 ) if and only if $u_{i}^{!} \mathcal{E}$ is semi-simple (resp. irreducible or 0 ) for any $i$.

Proof. Copy the proof of $\left[\mathrm{BGK}^{+} 87\right.$, VII.10.6].

\subsection{Local theory on formal disk}

This subsection is aimed to describe some cohomological functors for formal disk in terms of solution data à la Crew. These results clarify the relation between some computations of rigid cohomology and that of arithmetic $\mathcal{D}$-modules. In this subsection, we consider situation (A) in Notation and convention, and moreover, we assume $k$ contains the field with $q$ elements. We remark that this condition is satisfied in situation (B).

We denote by $K^{\text {ur }}$ the maximal unramified extension of $K$. We put $\mathcal{K}:=k((x))$, and let $G_{\mathcal{K}}$ be the absolute Galois group of $k((x))$. We often denote $G_{\mathcal{K}}$ by $G$, and the inertia group of $G$ by $I$. For an integer $n$ and a $\varphi$ - $K^{\text {ur }}$-vector space $V, V(n)$ denotes the $n$-th Tate twist of $V$, namely the underlying vector space is $V$ itself and the endomorphism is multiplied by $q^{-n}$ (cf. [Abe13, 2.7]).

Definition 1.5.1 ([Mar08, 3.1.3]). A Deligne module $D$ is a finite dimensional $\varphi-K^{\mathrm{ur}}$-vector space $(V, \varphi)$ endowed with semi-linear $G_{\mathcal{K}}$-action $\sigma$ commuting with the Frobenius action such that $\left.\sigma\right|_{I}$ factors through a finite index subgroup of $I$, and a linear homomorphism of $\varphi$ - $K^{\text {ur }}$-vector spaces $N: V(1) \rightarrow V$ which is nilpotent and equivariant. A homomorphism between Deligne modules is a homomorphism compatible with other data in the obvious way. We denote such a Deligne module by $(V, \sigma, \varphi, N)$, and the category of Deligne modules is denoted by Del. We sometimes denote $\sigma$ (resp. $\varphi, N)$ by $\sigma_{D}\left(\right.$ resp. $\varphi_{D}, N_{D}$ ). For an integer $n$, we define the $n$-th Tate twist $D(n)$ of $D$ by the quadruple $\left(V, q^{-n} \cdot \varphi, \sigma, N\right)$.

1.5.2. Let $(V, \varphi, \sigma, N)$ be a Deligne module. We will construct a canonical decomposition. The action of $I$ on $K^{\text {ur }}$ is trivial by definition, and in particular, the action of $I$ on $V$ is linear. We denote by $V^{I=1}$ the subset of $V$ fixed by the action of $I$. Since the action of $I$ on $V$ is linear, $V^{I=1}$ is in fact a $K^{\text {ur }}$-vector space. Now, by the definition of Deligne module, there exists a subgroup $I^{\prime} \subset I$ of finite index such that the action of $I^{\prime}$ on $V$ is trivial, and thus, this induces the linear action of $I / I^{\prime}$ on $V$. For any $x \in V, \sum_{\sigma \in I / I^{\prime}} \sigma(x)$ is

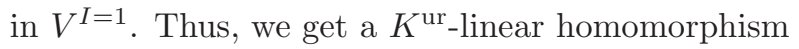

$$
\pi:=\left(\# I / I^{\prime}\right)^{-1} \sum_{\sigma \in I / I^{\prime}} \sigma: V \rightarrow V^{I=1} .
$$

We see easily that this does not depend on the choice of $I^{\prime}$. For any $\tau \in G$, we have $\tau \cdot I=I \cdot \tau$. Thus we get, for any $x \in V, \tau\left(\left(\sum_{\sigma \in I / I^{\prime}} \sigma\right)(x)\right)=\left(\sum_{\sigma \in I / I^{\prime}} \sigma\right)(\tau(x))$, which implies that $V^{I=1}$ possess a semi-linear action 
of $G$, which is nothing but the restriction of the $G$-action on $V$ to $V^{I=1}$, and $\pi$ commutes with $G$-actions. Since $\varphi$ and $N$ commute with $G$-action on $V$, these induce Frobenius and nilpotent operator on $V^{I=1}$, which is also denoted by $\varphi$ and $N$. Summing up, the quadruple $\left(V^{I=1}, \varphi, \sigma, N\right)$ form a Deligne module, and $\pi$ defines a homomorphism of Deligne modules.

By definition, the canonical inclusion $V^{I=1} \hookrightarrow V$ induces a homomorphism of Deligne modules, and $\pi$ is a section of this inclusion. Thus, we obtain a canonical decomposition of Deligne modules

$$
V \underset{(\pi, c)}{\stackrel{\sim}{\longrightarrow}} V^{I=1} \oplus V / V^{I=1}
$$

where $c: V \rightarrow V / V^{I=1}$ is the projection. We denote $V / V^{I=1}$ by $V^{I \neq 1}$, and consider this as a submodule of $V$.

Definition 1.5.3 ([Cre12, 6.1]). A solution data is a set $\left(\Psi, \Phi, c, v,\{v(\sigma)\}_{\sigma \in I}\right)$, where $\Psi$, $\Phi$ are Deligne modules, $c: \Psi \rightarrow \Phi$ (canonical homomorphism), $v: \Phi(1) \rightarrow \Psi$ (variation homomorphism), and $v(\sigma): \Phi \rightarrow \Psi$ (Galois variation homomorphism) are homomorphisms of Deligne modules such that

1. $N_{\Psi}=v \circ c$ and $N_{\Phi}=c \circ v$;

2. $\sigma_{\Psi}=1+v(\sigma) \circ c=$ and $\sigma_{\Phi}=1+c \circ v(\sigma)$.

A homomorphism between solution data $(\Psi, \Phi, c, v,\{v(\sigma)\})$ and $\left(\Psi^{\prime}, \Phi^{\prime}, c^{\prime}, v^{\prime},\left\{v^{\prime}(\sigma)\right\}\right)$ is a pair of homomorphisms $\left(f, f^{\prime}\right):(\Psi, \Phi) \rightarrow\left(\Psi^{\prime}, \Phi^{\prime}\right)$ which is compatible with $c, c^{\prime}, v, v^{\prime}, v(\sigma), v^{\prime}(\sigma)$ in the obvious way. We denote the category, in fact an abelian category, of solution data by Sol.

Let $V$ be a Deligne module such that $\sigma_{V}$ is trivial for any $\sigma \in I$. Then $(0, V, 0,0,\{0\})$ defines a solution data. This solution data is denoted by $i_{+}(V)$.

1.5.4. One of the most important results of [Cre06] is that there exists an equivalence between the category of holonomic $F-\mathcal{D}^{\text {an}}$-modules and that of solution data. Let $\mathcal{S}:=\operatorname{Spf}(R \llbracket x \rrbracket)$, a formal disk. Crew defined the ring of analytic differential operators $\mathcal{D}_{\mathcal{S}, \mathbb{Q}}^{\text {an }}$ and that with poles $\mathcal{D}_{\mathcal{S}, \mathbb{Q}}^{\text {an }}(0)$. For simplicity, we denote these rings by $\mathcal{D}^{\text {an }}$ and $\mathcal{D}^{\text {an }}(0)$. He proved fundamental properties of these rings. For the details, see $i b i d$.. Let $\mathcal{M}$ be a holonomic $F$ - $\mathcal{D}^{\text {an }}$-module. He defined ${ }^{(6)}$ two functors $\Psi$ and $\Phi$ by

$$
\Psi(\mathcal{M}):=\operatorname{Hom}_{\mathcal{D}} \text { an }(\mathbb{D}(\mathcal{M}), \mathcal{B}), \quad \Phi(\mathcal{M}):=\operatorname{Hom}_{\mathcal{D}} \text { an }(\mathbb{D}(\mathcal{M}), \mathcal{C})
$$

where $\mathcal{B}$ is the ring of hyperfunctions and $\mathcal{C}$ is the microfunction space (cf. [ibid., 1.4, 6.1]). These are naturally Deligne modules, and the canonical homomorphism $c: \mathcal{B} \rightarrow \mathcal{C}$ and variation homomorphism $v: \mathcal{C} \rightarrow \mathcal{B}$ define canonical and variation homomorphisms between $\Psi(\mathcal{M})$ and $\Phi(\mathcal{M})$, which gives us a solution data $(\Psi(\mathcal{M}), \Phi(\mathcal{M}), \ldots)$, and denoted by $\operatorname{Da}(\mathcal{M})$. For the precise construction, see [ibid., §6].

Now, let us denote by $\operatorname{Hol}\left(\mathcal{D}_{\mathcal{S}, \mathbb{Q}}^{\text {an }}(0)\right)$ the category of holonomic $F-\mathcal{D}_{\mathcal{S}, \mathbb{Q}}^{\text {an }}(0)$-modules, which is a subcat-

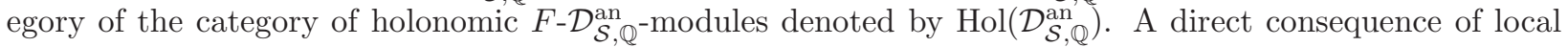
monodromy theorem is that the functor

$$
\Psi: \operatorname{Hol}\left(\mathcal{D}_{\mathcal{S}, \mathbb{Q}}^{\mathrm{an}}(0)\right) \rightarrow \operatorname{Del}
$$

induces an equivalence of categories. Now, one of the main theorems of the Crew's paper is the following:

Theorem 1.5.5 ([Cre12, 7.1.1]). The functor

$$
\operatorname{Da}: \operatorname{Hol}\left(\mathcal{D}_{\mathcal{S}, \mathbb{Q}}^{\text {an }}\right) \rightarrow \operatorname{Sol}
$$

induces an equivalence of categories.

A goal of this subsection is to describe $j_{!} \mathcal{M}$ and $j_{+} \mathcal{M}$ in terms of the solution data for a holonomic $F$ - $\mathcal{D}^{\text {an }}(0)$-module $\mathcal{M}$.

\footnotetext{
${ }^{(6)}$ Actually, he defined functors $\mathbb{V}$ and $\mathbb{W}$. We modify the definition slightly after [AM11].
} 
1.5.6. Let $\Psi:=(\Psi, \sigma, \varphi, N)$ be a Deligne module. Then the data $(\Psi, \Psi, \mathrm{id}, N,\{\sigma-1\})$ defines a solution data, and we denote this by $j_{!}(\Psi)$.

We are able to construct another solution data out of $\Psi$. We put $\Psi^{\prime}:=\Psi^{I=1}(-1) \oplus \Psi^{I \neq 1}$. Let

$$
\begin{gathered}
c: \Psi \cong \Psi^{I=1} \oplus \Psi^{I \neq 1} \stackrel{N \oplus \mathrm{id}}{\longrightarrow} \Psi^{I=1}(-1) \oplus \Psi^{I \neq 1} \cong \Psi^{\prime}, \\
v: \Psi^{\prime}(1) \cong \Psi^{I=1} \oplus \Psi^{I \neq 1}(1) \stackrel{\operatorname{id} \oplus N}{\longrightarrow} \Psi^{I=1} \oplus \Psi^{I \neq 1} \cong \Psi \\
v(\sigma): \Psi^{\prime} \cong \Psi^{I=1}(-1) \oplus \Psi^{I \neq 1} \stackrel{0 \oplus(\sigma-1)}{\longrightarrow} \Psi^{I=1} \oplus \Psi^{I \neq 1} \cong \Psi .
\end{gathered}
$$

Then we can check that $\left(\Psi, \Psi^{\prime}, c, v,\{v(\sigma)\}\right)$ defines a solution data. We denote this data by $j_{+}(\Psi)$. We can check that the homomorphisms of pairs (id, $c):(\Psi, \Psi) \rightarrow\left(\Psi, \Psi^{\prime}\right)$ induces a canonical homomorphism of solution data $j_{!}(\Psi) \rightarrow j_{+}(\Psi)$.

Let $\Psi^{I=1, N=0}:=\operatorname{Ker}\left(N: \Psi^{I=1} \rightarrow \Psi^{I=1}(-1)\right)$, which is a Deligne module. We have the following exact sequence of solution data:

$$
0 \rightarrow i_{+}\left(\Psi^{I=1, N=0}\right) \rightarrow j_{!}(\Psi) \rightarrow j_{+}(\Psi) \rightarrow i_{+}\left(\Psi^{I=1}(-1) / N \Psi^{I=1}\right) \rightarrow 0 .
$$

Proposition 1.5.7. Let $D:=(\widetilde{\Psi}, \widetilde{\Phi}, c, v,\{v(\sigma)\})$ be a solution data. There exists a canonical isomorphism

$$
\text { adj: } \operatorname{Hom}_{\mathrm{Del}}(\widetilde{\Psi}, \Psi) \stackrel{\sim}{\longrightarrow} \operatorname{Hom}_{\mathrm{Sol}}\left(D, j_{+}(\Psi)\right) \text {. }
$$

Proof. Let $c^{\prime}:=\left.c\right|_{\widetilde{\Psi}^{I \neq 1}}: \widetilde{\Psi}^{I \neq 1} \rightarrow \widetilde{\Phi}^{I \neq 1}$. Let us show that $c^{\prime}$ is an isomorphism. First, let us see the injectivity. Assume it were not injective, and let $x \in \widetilde{\Psi}^{I \neq 1}$ be a non-zero element in $\operatorname{Ker}\left(c^{\prime}\right)$. Then by assumption, there exists $\sigma \in I$ such that $\sigma(x) \neq x$. On the other hand, $\sigma(x)=\left(1+v(\sigma) \circ c^{\prime}\right)(x)=x$, which is a contradiction. Now, to show the claim, we construct the inverse $v^{\prime}$ of $c^{\prime}$. Let $I^{\prime}$ be the finite index subgroup of $I$ which acts trivially on $\widetilde{\Phi}^{I \neq 1}$. We put

$$
v^{\prime}:=\left(\# I / I^{\prime}\right)^{-1} \sum_{\sigma \in I / I^{\prime}} v(\sigma): \widetilde{\Phi}^{I \neq 1} \rightarrow \widetilde{\Psi}^{I \neq 1}
$$

Then, we see that

$$
c^{\prime} \circ v^{\prime}(x)=\left(\# I / I^{\prime}\right)^{-1}\left(\sum_{\sigma \in I / I^{\prime}} x-\sigma(x)\right)=x
$$

where the last equality holds since there are no trivial subrepresentation of $I$ in $\widetilde{\Phi}^{I \neq 1}$. Since $c^{\prime}$ is an injection, $v^{\prime}$ is the inverse of $c^{\prime}$. Thus the claim follows.

With these preparations, let us construct the homomorphism adj. Assume given a homomorphism $f: \widetilde{\Psi} \rightarrow$ $\Psi$. We put

$$
v_{1}: \widetilde{\Phi}^{I=1} \stackrel{v}{\rightarrow} \widetilde{\Psi}^{I=1}(-1) \stackrel{f}{\rightarrow} \Psi^{I=1}(-1), \quad v_{2}: \widetilde{\Phi}^{I \neq 1} \stackrel{v^{\prime}}{\rightarrow} \widetilde{\Psi}^{I \neq 1} \stackrel{f}{\rightarrow} \Psi^{I \neq 1}
$$

These homomorphisms define a homomorphism

$$
f^{\prime}: \widetilde{\Phi} \cong \widetilde{\Phi}^{I=1} \oplus \widetilde{\Phi}^{I \neq 1} \stackrel{v_{1} \oplus v_{2}}{\longrightarrow} \Psi^{I=1}(-1) \oplus \Psi^{I \neq 1}=\Psi^{\prime}
$$

This defines the following homomorphism of solution data:

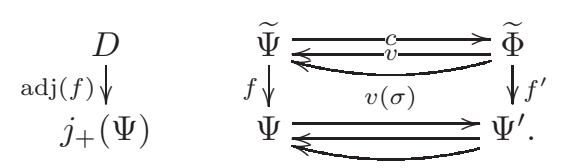

The verification of the compatibilities between homomorphisms is straightforward. As a result, we have a homomorphism

$$
\operatorname{adj}: \operatorname{Hom}(\widetilde{\Psi}, \Psi) \rightarrow \operatorname{Hom}\left(D, j_{+}(\Psi)\right)
$$

It is easy to check that this defines an isomorphism, and the details are left to the reader. 
1.5.8. Let $\mathcal{R}_{K}$ or simply $\mathcal{R}$ be the Robba ring over $K$. Let $\mathcal{M}$ be a finite free differential $\mathcal{R}$-module with Frobenius structure. Consider the following complex:

$$
0 \rightarrow \mathcal{M} \stackrel{\nabla}{\rightarrow} \mathcal{M} \otimes \Omega_{\mathcal{R}}^{1} \rightarrow 0
$$

where $\mathcal{M}$ is placed at degree 0 . We denote the $i$-th cohomology group by $H_{\text {loc }}^{i}(\mathcal{M})$. A result of ChristolMebkhout [CM01] shows that these cohomology groups are finite dimensional vector space over $K$ with the same dimension for $i=0,1$. Moreover, the canonical pairing

$$
H_{\mathrm{loc}}^{0}(\mathcal{M}) \otimes H_{\mathrm{loc}}^{1}\left(\mathcal{M}^{\vee}\right) \rightarrow H_{\mathrm{loc}}^{1}(\mathcal{R}) \cong K(-1)
$$

is perfect from [Cre98, §5]. Finally let us remark that our $H_{\mathrm{loc}}^{0}(M \otimes A(x))$ is denoted by $H^{0}(M \otimes A(x))$ by Crew in $[$ Cre98, 10.7].

Theorem 1.5.9. Let $\mathcal{M}$ be a holonomic $F-\mathcal{D}^{\text {an }}(0)$-module.

(i) We have $\Psi:=\Psi(\mathcal{M}) \cong\left(\mathcal{B} \otimes_{\mathcal{R}} \mathcal{M}\right)^{\partial=0}(1)$.

(ii) We have

$$
\operatorname{Da}\left(j_{!}(\mathcal{M})\right) \cong j_{!}(\Psi(\mathcal{M})), \quad \operatorname{Da}\left(j_{+}(\mathcal{M})\right) \cong j_{+}(\Psi(\mathcal{M})) .
$$

(iii) We have the following isomorphisms of $\varphi$ - $K^{\mathrm{ur}}$-vector spaces with semi-linear $\mathrm{Gal}(\bar{k} / k)$-action:

$$
\begin{gathered}
H_{\mathrm{loc}}^{0}(\mathcal{M})(1) \otimes K^{\mathrm{ur}} \cong H^{-1}\left(i^{+} j_{+}(\mathcal{M})\right) \otimes K^{\mathrm{ur}} \cong \Psi^{I=1, N=0} \\
H_{\mathrm{loc}}^{1}(\mathcal{M})(1) \otimes K^{\mathrm{ur}} \cong H^{0}\left(i^{+} j_{+}(\mathcal{M})\right) \otimes K^{\mathrm{ur}} \cong \Psi^{I=1}(-1) / N \Psi^{I=1}
\end{gathered}
$$

Proof. Let us show (i). We have

$$
\Psi:=\operatorname{Hom}_{\mathcal{D}} \text { an }(\mathbb{D}(\mathcal{M}), \mathcal{B}) \cong \operatorname{Hom}_{\mathcal{D} \text { an }(0)}(\mathbb{D}(\mathcal{M}), \mathcal{B}) .
$$

By using $\left[\right.$ Abe13, 3.12], we see that $\mathbb{D}(\mathcal{M}) \cong \mathcal{M}^{\vee}(-1)$. Thus, we get

$$
\operatorname{Hom}_{\mathcal{D}^{\text {an }}(0)}(\mathbb{D}(\mathcal{M}), \mathcal{B}) \cong \operatorname{Hom}_{\mathcal{D}^{\text {an }}(0)}\left(\mathcal{M}^{\vee}, \mathcal{B}\right)(1) \cong \operatorname{Hom}_{\mathcal{D}^{\text {an }}(0)}(\mathcal{R}, \mathcal{M} \otimes \mathcal{B})(1),
$$

and the first claim follows.

By $\left[\right.$ Cre12, 6.1.2], $\mathrm{Da}\left(j_{!}(\mathcal{M})\right) \cong j_{!}(\Psi)$. Moreover, by Proposition 1.5.7, $\mathrm{Da}\left(j_{+}(\mathcal{M})\right)$ is canonically isomorphic to $j_{+}(\Psi)$, and (ii) follows. Now, we have

$$
\operatorname{Hom}\left(\mathbb{D}\left(j_{+} \mathcal{M}\right), \mathcal{O}_{K^{\mathrm{ur}}}^{\mathrm{an}}\right) \cong H_{\mathrm{loc}}^{0}(\mathcal{M})(1), \quad \operatorname{Ext}^{1}\left(\mathbb{D}\left(j_{+} \mathcal{M}\right), \mathcal{O}_{K^{\mathrm{ur}}}^{\mathrm{an}}\right) \cong H_{\mathrm{loc}}^{1}(\mathcal{M})(1),
$$

by $[$ AM11, 3.1.10]. We have the following diagram of exact sequences:

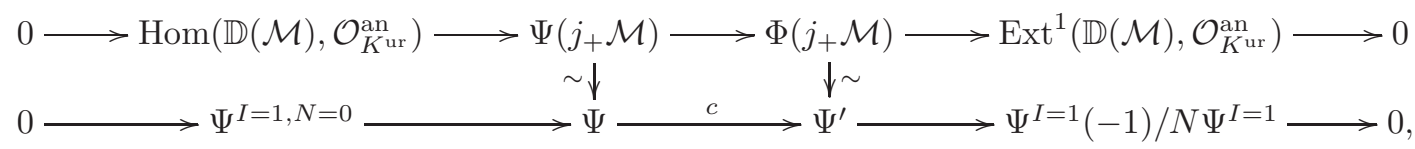

where the first row is exact by [Cre12, 6.1.1]. These show that

$$
H_{\mathrm{loc}}^{0}(\mathcal{M})(1) \otimes K^{\mathrm{ur}} \cong \Psi^{I=1, N=0}, \quad H_{\mathrm{loc}}^{1}(\mathcal{M})(1) \otimes K^{\mathrm{ur}} \cong \Psi^{I=1}(-1) / N \Psi^{I=1} .
$$

Finally, considering the following exact sequence, we conclude the proof.

$$
0 \rightarrow i_{+} H^{-1}\left(i^{+} j_{+}(\mathcal{M})\right) \rightarrow j_{!}(\mathcal{M}) \rightarrow j_{+}(\mathcal{M}) \rightarrow i_{+} H^{0}\left(i^{+} j_{+}(\mathcal{M})\right) \rightarrow 0 .
$$




\section{Mixed complexes}

In this section, we define "mixed $F$-complexes", and prove some basic properties. We consider situation (B) in Notation and convention. We define the notion of mixedness in the first subsection, followed by definition of mixedness for any overholonomic $F$-complexes in the next subsection. The last subsection is aimed to estimate some weights which is used in the proof of our main theorem.

\subsection{Mixedness for $F$-isocrystals}

2.1.1. In this subsection, let $(Y, X, \mathcal{P})$ be a frame such that $Y$ is smooth. Let $y$ be a closed point of $Y$ with residue field $k(y)$. By abuse of notation, we denote by $y$ the frame $(\operatorname{Spec}(k(y))$, $\operatorname{Spec}(k(y)), \mathcal{P})$, and by $i_{y}$ the canonical morphism of frames $y \rightarrow(Y, X, \mathcal{P})$. We recall the notation of paragraph 1.1.8 (iii).

We start by recalling the following definition of $\iota$-weight by Deligne [Del80]:

Definition 2.1.2. (i) Let $w \in \mathbb{R}$. A number $\alpha$ in $\overline{\mathbb{Q}}_{p}$ is said to be of $\iota$-weight $w$ if

$$
|\iota(\alpha)|=q^{w / 2} .
$$

(ii) Let $\varphi$-Vect $K$ be the category of pairs $(V, \varphi)$ where $V$ is a finite dimensional $K$-vector space and $\varphi$ is an automorphism of $V$. Let $(a, a, f):\left(\operatorname{Spec}\left(k^{\prime}\right), \operatorname{Spec}\left(k^{\prime}\right), \mathcal{P}^{\prime}\right) \rightarrow(\operatorname{Spec}(k), \operatorname{Spec}(k), \operatorname{Spf} \mathcal{V})$ be a morphism of frames such that $a$ is finite and $f$ is the structural morphism of $\mathcal{P}^{\prime}$. We have the canonical functor $f_{+}: F$-Ovhol $\left(\operatorname{Spec}\left(k^{\prime}\right), \mathcal{P}^{\prime} / K\right) \rightarrow \varphi$-Vect $K$. Let $\mathcal{E}^{\prime} \in F-D_{\text {ovhol }}^{\mathrm{b}}\left(\operatorname{Spec}\left(k^{\prime}\right), \mathcal{P}^{\prime} / K\right)$. We say that $\mathcal{E}^{\prime}$ is $\iota$-pure if there exists a real number $w$, the weight of $\mathcal{E}^{\prime}$, such that any eigenvalue $\alpha$ of the automorphism on $f_{+}\left(\mathcal{H}^{j}\left(\mathcal{E}^{\prime}\right)\right)$ is of $\iota$-weight equal to $w+j$, for any integer $j$.

This definition is independent of the choice of $\mathcal{P}^{\prime}$, i.e., if $\rho=(\mathrm{id}, \mathrm{id}, \star):\left(\operatorname{Spec}\left(k^{\prime}\right), \operatorname{Spec}\left(k^{\prime}\right), \mathcal{P}^{\prime \prime}\right) \rightarrow$ $\left(\operatorname{Spec}\left(k^{\prime}\right), \operatorname{Spec}\left(k^{\prime}\right), \mathcal{P}^{\prime}\right)$ is a morphism of frames, then $\rho^{!}$and $\rho_{+}$induces quasi-inverse equivalences of categories between $\iota$-pure of weight $w$ objects of $F$ - $D_{\text {ovhol }}^{\mathrm{b}}\left(\operatorname{Spec}\left(k^{\prime}\right), \mathcal{P}^{\prime} / K\right)$ and $\iota$-pure of weight $w$ objects of $F-D_{\text {ovhol }}^{\mathrm{b}}\left(\operatorname{Spec}\left(k^{\prime}\right), \mathcal{P}^{\prime \prime} / K\right)$.

Definition 2.1.3. Let $\mathcal{E} \in F$-Isoc ${ }^{\dagger \dagger}(Y, \mathcal{P} / K$ ) (see notation 1.2.14).

1. We say that $\mathcal{E}$ is $\iota$-pure if there exists a real number $w$, called the weight of $\mathcal{E}$, such that for any closed point $x$ of $Y$ the pull-back $i_{x}^{+} \mathcal{E}$ is $\iota$-pure of weight $w$.

2. We say that $\mathcal{E}$ is $\iota$-mixed if all the constituents (i.e. irreducible subquotients) of $\mathcal{E}$ are $\iota$-pure.

3. We say that $\mathcal{E}$ is $\iota$-mixed of weight $\geq w($ resp. $\leq w)$ if $\mathcal{E}$ is $\iota$-mixed and if the weights of all the constituents of $\mathcal{E}$ are $\geq w($ resp. $\leq w)$.

Remark 2.1.4. Let $E \in F$-Isoc ${ }^{\dagger}(Y, X / K)$ be an overconvergent $F$-isocrystal and $\mathcal{E}:=\operatorname{sp}_{X \hookrightarrow \mathcal{P},+}(E)$ be the corresponding object of $F$-Isoc ${ }^{\dagger \dagger}(Y, \mathcal{P} / K)$. We assume that $Y$ is pure of dimension $d_{Y}$. The $F$-isocrystal $\mathcal{E}$ is $\iota$-pure of weight ${ }^{(7)} w$ if and only if $E$ is $\iota$-pure of weight $w+d_{Y}$ in the sense of Crew or Kedlaya (see $[\operatorname{Ked} 06,5.1])$ by $(1.2 .14 .1)$.

Remark 2.1.5. Let $\mathcal{E} \in F$-Isoc ${ }^{\dagger \dagger}(Y, \mathcal{P} / K)$ and let $\mathcal{E}^{\prime}$ be a constituent of $\mathcal{E}$ considered as an object of $\operatorname{Isoc}^{\dagger \dagger}(\mathcal{P}, X, Y)$ (without Frobenius). Then $\mathcal{E}^{\prime}$ automatically possesses a Frobenius structure, namely we have an isomorphism $\mathcal{E}^{\prime} \stackrel{\sim}{\rightarrow} F^{* N}\left(\mathcal{E}^{\prime}\right)$ for some positive integer $N$. The argument is the same as [CM01, $6.0-15]$.

Lemma 2.1.6. Let $\mathcal{E} \in F-\operatorname{Isoc}^{\dagger \dagger}(Y, \mathcal{P} / K)$.

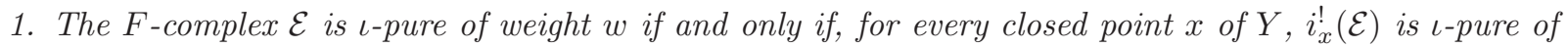
weight $w$.

\footnotetext{
${ }^{(7)}$ We notice that there is a typo in the definition [Car06, 8.3.2]. Indeed, $w-d_{Y_{i}}$ should be replaced by $w+d_{Y_{i}}$.
} 
2. Let $f:\left(Y^{\prime}, X^{\prime}, \mathcal{P}^{\prime}\right) \rightarrow(Y, X, \mathcal{P})$ be a morphism of frames such that $Y^{\prime}$ is smooth as well. If $\mathcal{E}$ is $\iota$-mixed of weight $\leq w($ resp. $\geq w)$ then so are $f^{+} \mathcal{E}$ and $f^{!} \mathcal{E}$.

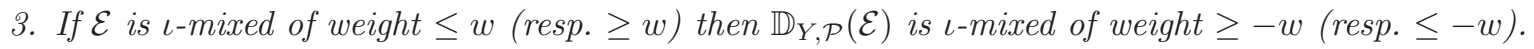

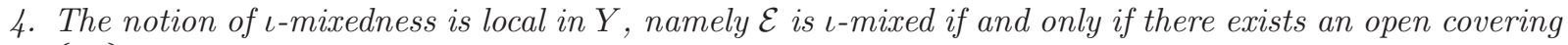
$\left\{Y_{i}\right\}$ of $Y$ such that $\mathcal{E} \|_{Y_{i}}$ is ı-mixed for any $i$.

Proof. The first statement follows from the isomorphism (1.2.14.1). Let us check the second claim. It suffices to show that if $\mathcal{E}$ is $\iota$-pure of weight $w$, so are $f^{+} \mathcal{E}$ and $f^{!} \mathcal{E}$. Let $x^{\prime}$ be a closed point of $Y^{\prime}$. Then to check the purity of $f^{+} \mathcal{E}$ (resp. $f^{!} \mathcal{E}$ ), it suffices to check the purity for $i_{x^{\prime}}^{+} f^{+} \mathcal{E}$ (resp. $i_{x^{\prime}}^{!} f^{!} \mathcal{E}$ ) by definition (resp. by the first claim). These follow by the transitivity of $(\cdot)^{+}$and $(\cdot)^{!}$. The third claim follows by $i_{x}^{+} \circ \mathbb{D} \cong \mathbb{D} \circ i_{x}^{!}$ combining with 1. The last one is a consequence of the Lemma 1.4.6.

Lemma 2.1.7. Let $0 \rightarrow \mathcal{E}^{\prime} \rightarrow \mathcal{E} \rightarrow \mathcal{E}^{\prime \prime} \rightarrow 0$ be an exact sequence in $F$-Isoc ${ }^{\dagger \dagger}(Y, \mathcal{P} / K)$. The overconvergent

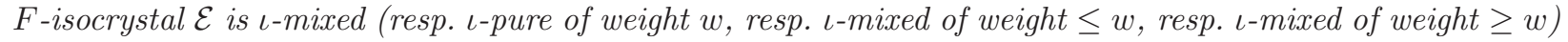
if and only if so are $\mathcal{E}^{\prime}$ and $\mathcal{E}^{\prime \prime}$.

Proof. Let $\operatorname{gr}(\mathcal{E})$ be the direct sum of the constituents of $\mathcal{E}$, which is defined up to (non-canonical) isomorphism. We can check easily that $\operatorname{gr}(\mathcal{E}) \stackrel{\sim}{\longrightarrow} \operatorname{gr}\left(\mathcal{E}^{\prime}\right) \oplus \operatorname{gr}\left(\mathcal{E}^{\prime \prime}\right)$.

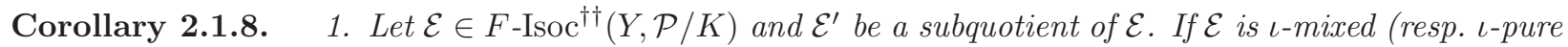

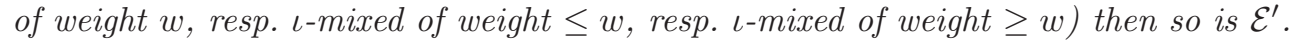

2. Let $\mathcal{E}^{\prime} \rightarrow \mathcal{E} \rightarrow \mathcal{E}^{\prime \prime}$ be an exact sequence in $F$-Isoc ${ }^{\dagger \dagger}(Y, \mathcal{P} / K)$. If the overconvergent $F$-isocrystal $\mathcal{E}^{\prime}, \mathcal{E}^{\prime \prime}$

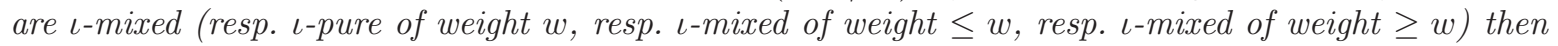
so is $\mathcal{E}$.

Proof. These are consequences of Lemma 2.1.7.

Definition 2.1.9. $\quad 1$. We say that $\mathcal{E} \in F$ - $D_{\text {isoc }}^{\mathrm{b}}(Y, \mathcal{P} / K)$ (see notation 1.2.14) is $\iota$-mixed if $\mathcal{H}_{\mathrm{t}}^{j}(\mathcal{E})$ is $\iota$ mixed for any integer $j$. We denote by $F-D_{\text {isoc, } \mathrm{m}}^{\mathrm{b}}(Y, \mathcal{P} / K)$ the full subcategory of $F-D_{\text {isoc }}^{\mathrm{b}}(Y, \mathcal{P} / K)$ consisting of $\iota$-mixed $F$-complexes.

2. We say that $\mathcal{E} \in F$ - $D_{\text {isoc }}^{\mathrm{b}}(Y, \mathcal{P} / K)$ is $\iota$-pure of weight $w$ (resp. $\iota$-mixed of weight $\geq w$, resp. $\iota$-mixed of weight $\leq w)$ if $\mathcal{H}_{\mathrm{t}}^{j}(\mathcal{E})$ is $\iota$-pure of weight $w+j$ (resp. $\iota$-mixed of weight $\geq w+j$, resp. $\iota$-mixed of weight $\leq w+j$ ) for any integer $j$.

Remark 2.1.10. Assume $Y$ is smooth, and let $\mathcal{U}$ be an open formal subscheme of $\mathcal{P}$ containing $Y$. Let $\mathcal{E} \in F$ - $D_{\text {ovhol }}^{\mathrm{b}}(Y, \mathcal{P} / K)$. The property " $\mathcal{E} \in F$ - $D_{\text {isoc }}^{\mathrm{b}}(Y, \mathcal{P} / K)$ and $\mathcal{E}$ is $\iota$-pure of weight $w$ " is equivalent

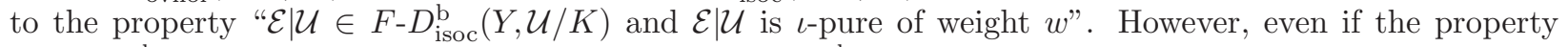
$\mathcal{E} \in F-D_{\text {isoc, } \mathrm{m}}^{\mathrm{b}}(Y, \mathcal{P} / K)$ implies the property $\mathcal{E} \mid \mathcal{U} \in F$ - $D_{\text {isoc, } \mathrm{m}}^{\mathrm{b}}(Y, \mathcal{U} / K)$, we do not know if the converse is true (see [Abe11, Remark 2.2]).

Remark 2.1.11. Suppose in this remark that $X$ is proper. Let $\mathcal{E} \in F-D_{\text {isoc }}^{\mathrm{b}}(Y, \mathcal{P} / K)$. Let $U$ be a dense open subvariety of $Y$ and $\mathcal{E} \| U \in F-D_{\text {isoc }}^{\mathrm{b}}(U, \mathcal{P} / K)$ the restriction on $(U, \mathcal{P} / K)$ of $\mathcal{E}$ (see notation 1.1.8.iii)). Then, it follows from [Cre98, 10.8] (or we can follow the $p$-adic analogue of the proof of the semicontinuity of weights of [KW01, I.2.8]) that $\mathcal{E}$ is $\iota$-pure of weight $w$ if and only if $\mathcal{E} \| U$ is $\iota$-pure of weight $w$. The hypothesis that $X$ is proper is useful in this proof since we use the $p$-adic cohomological interpretation of the $L$-function. We do not know what is true when $X$ is not proper.

Lemma 2.1.12. Let $\mathcal{E}^{\prime} \rightarrow \mathcal{E} \rightarrow \mathcal{E}^{\prime \prime} \stackrel{+}{\rightarrow}$ be an exact triangle in $F$ - $D_{\text {isoc }}^{\mathrm{b}}(Y, \mathcal{P} / K)$. If the $F$-complexes $\mathcal{E}^{\prime}$ and $\mathcal{E}^{\prime \prime}$ are $\iota$-mixed (resp. $\iota$-pure of weight $w$, resp. $\iota$-mixed of weight $\leq w$, resp. $\iota$-mixed of weight $\geq w$ ), then so is $\mathcal{E}$.

Proof. This is a consequence of Lemma 2.1.8.2. 


\subsection{Mixedness for overholonomic F-complexes}

In this subsection, we define our one of the main players, $\iota$-mixed $F$-complexes, and show some first properties. We continue to let $(Y, X, \mathcal{P})$ be a frame.

2.2.1. Recall from [Car08, 4.1.2] that an ordered set of subschemes $\left\{Y_{i}\right\}_{i=1, \ldots, r}$ of $Y$ is said to be a smooth stratification if the following holds: $1 .\left\{Y_{i}\right\}$ is a stratification, namely putting $Y_{0}:=\emptyset, Y_{k}$ is an open subscheme of $Y \backslash \bigcup_{i<k} Y_{i}$ and $Y=\bigcup_{1 \leq i \leq r} Y_{i}$. 2. $Y_{i}$ is a smooth variety. A stratification $\left\{Y_{j}^{\prime}\right\}_{1 \leq j \leq r^{\prime}}$ is said to be a refinement of $\left\{Y_{i}\right\}$ if there exists an increasing function $\phi:[1, r] \cap \mathbb{N} \rightarrow\left[1, r^{\prime}\right] \cap \mathbb{N}$ such that $\bigcup_{i \leq k} Y_{i}=\bigcup_{j \leq \phi(k)} Y_{j}^{\prime}$ for any $1 \leq k \leq r$. The following facts are useful:

$\left(^{*}\right)$ For any stratification $\left\{Y_{i}\right\}_{1 \leq i \leq r}$ of $Y$, there is a smooth stratification $\left\{Y_{j}^{\prime}\right\}_{1 \leq j \leq r^{\prime}}$, which is a refinement of $\left\{Y_{i}\right\}$. Moreover, given two stratifications $\left\{Y_{i}\right\}$ and $\left\{Y_{j}^{\prime}\right\}$, there exists a stratification $\left\{Y_{k}^{\prime \prime}\right\}$ which is a refinement of both $\left\{Y_{i}\right\}$ and $\left\{Y_{j}^{\prime}\right\}$.

Definition 2.2.2. Let $\mathcal{E} \in F-D_{\text {ovhol }}^{\mathrm{b}}(Y, \mathcal{P} / K)$.

1. We say that $\mathcal{E}$ is $\iota$-mixed if there exists a smooth stratification $\left\{Y_{i}\right\}_{1 \leq i \leq r}$ of $Y$ such that $\mathcal{E} \|_{Y_{i}}$ is in $F$ - $D_{\text {isoc, } \mathrm{m}}^{\mathrm{b}}\left(Y_{i}, \mathcal{P} / K\right)$ for any $i=1, \ldots, r$. We denote by $F-D_{\mathrm{m}}^{\mathrm{b}}(Y, \mathcal{P} / K)$ the full subcategory of $F$ - $D_{\text {ovhol }}^{\mathrm{b}}(Y, \mathcal{P} / K)$ consisting of $\iota$-mixed $F$-complexes.

2. We say that $\mathcal{E}$ is $\iota$-mixed of weight $\leq w$ if $\mathcal{E}$ is $\iota$-mixed and, for every closed point $x$ in $Y$, the $F$-complex $i_{x}^{+}(\mathcal{E})$ is $\iota$-mixed of weight $\leq w$. We denote by $F-D_{\leq w}^{\mathrm{b}}(Y, \mathcal{P} / K)$ the full subcategory of $F-D_{\mathrm{m}}^{\mathrm{b}}(Y, \mathcal{P} / K)$ consisting of $\iota$-mixed $F$-complexes of weight $\leq w$.

3. We say that $\mathcal{E}$ is $\iota$-mixed of weight $\geq w$ if $\mathcal{E}$ is $\iota$-mixed and, for every closed point $x$ in $Y$, the $F$-complex $i_{x}^{!}(\mathcal{E})$ is $\iota$-mixed of weight $\geq w$. We denote by $F-D_{\geq w}^{\mathrm{b}}(Y, \mathcal{P} / K)$ the full subcategory of $F-D_{\mathrm{m}}^{\mathrm{b}}(Y, \mathcal{P} / K)$ consisting of $\iota$-mixed $F$-complexes of weight $\geq w$.

4. We say that $\mathcal{E}$ is $\iota$-pure of weight $w$ if $\mathcal{E}$ is both $\iota$-mixed of weight $\leq w$ and of weight $\geq w$. The $\iota$-weight of $\mathcal{E}$ is denoted by $\operatorname{wt}(\mathcal{E})$.

Remark 2.2.3. (i) Let $\mathcal{E} \in F-D_{\mathrm{m}}^{\mathrm{b}}(Y, \mathcal{P} / K)$. Then there exist real numbers $w_{1}, w_{2}$ such that $\mathcal{E}$ is of weight both $\geq w_{1}$ and $\leq w_{2}$. Indeed, for an $\iota$-pure overconvergent $F$-isocrystal, we may take $w_{1}=w_{2}$ to be its $\iota$-weight. For an $\iota$-mixed overconvergent $F$-isocrystal, the constituents are $\iota$-pure by definition, so the claim holds. In the general case, we may reduce to the $\iota$-mixed overconvergent $F$-isocrystal case by dévissage.

(ii) Let $\mathcal{E}$ be an $F$-complex in $F-D_{\mathrm{m}}^{\mathrm{b}}(Y, \mathcal{P} / K)$. Assume that $\mathbb{D}_{Y, \mathcal{P}}(\mathcal{E})$ is $\iota$-mixed as well. Then by definition, $\mathcal{E}$ is $\iota$-mixed of weight $\geq w($ resp. $\leq w)$ if and only if $\mathbb{D}_{Y, \mathcal{P}}(\mathcal{E})$ is $\iota$-mixed of weight $\leq-w$ (resp. $\geq-w)$. One of the main results of this paper is to show that, in fact, $\mathbb{D}_{Y, \mathcal{P}}(\mathcal{E})$ is $\iota$-mixed if $\mathcal{E}$ is. See Theorem 4.1.3 for the details.

(iii) Suppose that $X$ is proper. Let $\mathcal{E} \in F-D_{\text {isoc }}^{\mathrm{b}}(Y, \mathcal{P} / K)$. Then, it follows from the remark 2.1.11 that the $F$-complex $\mathcal{E}$ is $\iota$-mixed as an object of $F$ - $D_{\text {isoc }}^{\mathrm{b}}(Y, \mathcal{P} / K)$ if and only if $\mathcal{E}$ is $\iota$-mixed as an object of $F-D_{\text {ovhol }}^{\mathrm{b}}(Y, \mathcal{P} / K)$.

Proposition 2.2.4. Let $u:\left(Y^{\prime}, X^{\prime}, \mathcal{P}^{\prime}\right) \rightarrow(Y, X, \mathcal{P})$ be a morphism of frames, and let $\mathcal{E}$ be in $F-D_{\geq w}^{\mathrm{b}}(Y, \mathcal{P} / K)$. Then $u^{!}(\mathcal{E})$ is in $F-D_{\geq w}^{\mathrm{b}}\left(Y^{\prime}, \mathcal{P}^{\prime} / K\right)$.

Proof. Let $Y=\sqcup_{i=1, \ldots, r} Y_{i}$ be a smooth stratification in $P$ such that $\mathcal{E} \|_{Y_{i}} \in F-D_{\text {isoc, } \mathrm{m}}^{\mathrm{b}}\left(Y_{i}, \mathcal{P} / K\right)$ for any $i=$ $1, \ldots, r$. Let $b: Y^{\prime} \rightarrow Y$ be the morphism defined by $u$. We get the stratification $Y^{\prime}=\sqcup_{i=1, \ldots, r} b^{-1}\left(Y_{i}\right)$. By using 2.2.1 (*), there exists a smooth stratification of $Y^{\prime}$ in $P^{\prime}$ which is a refinement of $Y^{\prime}=\cup_{i=1, \ldots, r} b^{-1}\left(Y_{i}\right)$. With Lemma 2.1.6.2, we can conclude.

Lemma 2.2.5. Let $u=(\mathrm{id}, \star, \star, \star):\left(Y, X^{\prime}, \mathcal{P}^{\prime}, \mathcal{Q}^{\prime}\right) \rightarrow(Y, X, \mathcal{P}, \mathcal{Q})$ be a complete morphism of l.p. frames. Take $\mathcal{E} \in F-D_{\text {ovhol }}^{\mathrm{b}}(Y, \mathcal{P} / K), \mathcal{E}^{\prime} \in F-D_{\text {ovhol }}^{\mathrm{b}}\left(Y, \mathcal{P}^{\prime} / K\right)$, and choose $* \in\{\mathrm{m}, \geq w, \leq w\}$. 
1. Suppose $Y$ to be smooth. We get $\mathcal{E} \in F-D_{\mathrm{isoc}, *}^{\mathrm{b}}(Y, \mathcal{P} / K)$ if and only if $u^{!}(\mathcal{E}) \in F-D_{\mathrm{isoc}, *}^{\mathrm{b}}\left(Y, \mathcal{P}^{\prime} / K\right)$ (resp. $\mathcal{E}^{\prime} \in F-D_{\text {isoc,* }}^{\mathrm{b}}\left(Y, \mathcal{P}^{\prime} / K\right)$ if and only if $u_{+}\left(\mathcal{E}^{\prime}\right) \in F-D_{\text {isoc, }, *}^{\mathrm{b}}(Y, \mathcal{P} / K)$ ).

2. We have $\mathcal{E} \in F-D_{*}^{\mathrm{b}}(Y, \mathcal{P} / K)$ if and only if $u^{!}(\mathcal{E}) \in F-D_{*}^{\mathrm{b}}\left(Y, \mathcal{P}^{\prime} / K\right)$ (resp. $\mathcal{E}^{\prime} \in F-D_{*}^{\mathrm{b}}\left(Y, \mathcal{P}^{\prime} / K\right)$ if and only if $\left.u_{+}\left(\mathcal{E}^{\prime}\right) \in F-D_{*}^{\mathrm{b}}(Y, \mathcal{P} / K)\right)$.

Proof. From [Car12a, 2.5], the functors $u_{+}$and $u^{!}$induce canonical equivalence of categories between $F$ - $D_{\text {ovhol }}^{\mathrm{b}}(Y, \mathcal{P} / K)$ and $F-D_{\text {ovhol }}^{\mathrm{b}}\left(Y, \mathcal{P}^{\prime} / K\right)$. Hence, we reduce to checking the part concerning the functor $u^{!}$. From [Car12a, 2.5], we have the isomorphism $u^{!} \stackrel{\sim}{\rightarrow} u^{+}$. Thus, by transitivity of the (extraordinary) inverse image, the part concerning " $\leq w$ " or " $\geq w$ " is a consequence of that concerning " $\mathrm{m}$ ".

Let us check 1. It amounts to check that $\mathcal{E}$ is purely of weight $w$ if and only if so is $u^{+}(\mathcal{E})$. Take a closed point $y$ of $Y$. We denote the morphism $y \rightarrow\left(Y, X^{\prime}, \mathcal{P}^{\prime}, \mathcal{Q}^{\prime}\right)$ by $i_{y}^{\prime}$ to tell from the morphism $i_{y}: y \rightarrow(Y, X, \mathcal{P}, \mathcal{Q})$. Then $u \circ i_{y}^{\prime}=i_{y}$. Thus by transitivity of $(\cdot)^{+}$, the claim follows by definition. Let us check 2. The "only if" part follows from Proposition 2.2.4, so let us check the "if" part. Let $Y=\sqcup_{i=1, \ldots, r} Y_{i}$ be a smooth stratification of $Y$ such that for any $i=1, \ldots, r, u^{!}(\mathcal{E}) \|_{Y_{i}} \in F-D_{\text {isoc,m }}^{\mathrm{b}}\left(Y_{i}, \mathcal{P}^{\prime} / K\right)$. Since for any subvariety $Y^{\prime}$ of $Y$ appearing in this stratification we have $\mathbb{R} \underline{\Gamma}_{Y^{\prime}}^{\dagger}\left(u^{!}(\mathcal{E})\right) \stackrel{\sim}{\rightarrow} u^{!}\left(\mathbb{R} \underline{\Gamma}_{Y^{\prime}}^{\dagger}(\mathcal{E})\right)$, and we are reduced to 1 .

Lemma 2.2.6. Let $u=(j, a, g, f):\left(Y^{\prime}, X^{\prime}, \mathcal{P}^{\prime}, \mathcal{Q}^{\prime}\right) \rightarrow(Y, X, \mathcal{P}, \mathcal{Q})$ be a complete morphism of l.p. frames such that $j$ is an immersion. If $\mathcal{E}^{\prime}$ is an $F$-complex in $F-D_{\mathrm{m}}^{\mathrm{b}}\left(Y^{\prime}, \mathcal{P} / K\right)\left(\right.$ resp. $\left.F-D_{\geq w}^{\mathrm{b}}\left(Y^{\prime}, \mathcal{P} / K\right)\right)$ then $u_{+}\left(\mathcal{E}^{\prime}\right)$ is an $F$-complex in $F-D_{\mathrm{m}}^{\mathrm{b}}(Y, \mathcal{P} / K)$ (resp. $F$ - $\left.D_{\geq w}^{\mathrm{b}}(Y, \mathcal{P} / K)\right)$.

Proof. Let $X^{\prime \prime}$ be the closure of $Y^{\prime}$ in $X, c: X^{\prime} \rightarrow X^{\prime \prime}$ and $d: X^{\prime \prime} \rightarrow X$ the canonical factorization of $a$. We have $u=(j, d$, id, id) $\circ$ (id, $c, g, f)$. By Lemma 2.2.5, we reduce to the case where $u=(j, d$, id, id). In that case $u_{+}\left(\mathcal{E}^{\prime}\right)=\mathcal{E}^{\prime}$. Suppose that $Y^{\prime} \subset Y$ is closed. The we get the stratification $Y=\left(Y \backslash Y^{\prime}\right) \sqcup Y^{\prime}$. Let $Y^{\prime}=\sqcup_{i=1, \ldots, r} Y_{i}^{\prime}$ be a smooth stratification of $Y^{\prime}$ such that $\mathcal{E}^{\prime} \|_{Y_{i}^{\prime}} \in F-D_{\mathrm{isoc}, \mathrm{m}}^{\mathrm{b}}\left(Y_{i}^{\prime}, \mathcal{P} / K\right)$ for any $i=1, \ldots, r$. Applying 2.2.1 (*) to the trivial stratification of $Y \backslash Y^{\prime}$, there exist a smooth stratification $Y \backslash Y^{\prime}=\sqcup_{i=1, \ldots, s} Y_{i}^{\prime \prime}$. Thus, we get the smooth stratification $Y=\left(\sqcup_{i=1, \ldots, s} Y_{i}^{\prime \prime}\right) \sqcup\left(\sqcup_{i=1, \ldots, r} Y_{i}^{\prime}\right)$. Since $\mathcal{E}^{\prime} \|_{Y_{i}^{\prime \prime}}=0$, we get a desired stratification. Similarly, if $Y^{\prime} \subset Y$ is open, then we get the stratification $Y=Y^{\prime} \sqcup\left(Y^{2} \backslash Y^{\prime}\right)$ and we proceed as above by using $2.2 .1\left(^{*}\right)$.

Proposition 2.2.7. $\quad$ 1. Let $\mathcal{F}^{\prime} \rightarrow \mathcal{F} \rightarrow \mathcal{F}^{\prime \prime} \stackrel{+}{\rightarrow}$ be a distinguished triangle in $F$ - $D_{\text {ovhol }}^{\mathrm{b}}(Y, \mathcal{P} / K)$. If $\mathcal{F}^{\prime}$ and $\mathcal{F}^{\prime \prime}$ are $\iota$-mixed (resp. of weight $\geq w$, resp. of weight $\leq w$ ), then so is $\mathcal{F}$.

2. Let $\mathcal{E} \in F-D_{\mathrm{ovhol}}^{\mathrm{b}}(Y, \mathcal{P} / K)$. Let $Y=\sqcup_{i=1, \ldots, r} Y_{i}$ be a stratification of $Y$. The $F$-complex $\mathcal{E}$ is $\iota$-mixed (resp. $\iota$-mixed of $\iota$-weight $\geq w$ ) if and only if, for any $i=1, \ldots r$, so are the $F$-complexes $\mathbb{R} \underline{\Gamma}_{Y_{i}}^{\dagger}(\mathcal{E})$.

3. Let $\mathcal{E} \in F-D_{\text {ovhol }}^{\mathrm{b}}(Y, \mathcal{P} / K)$. The notion of $\iota$-mixedness is local in $Y$, namely $\mathcal{E}$ is $\iota$-mixed if and only if there exists an open covering $\left\{Y_{i}\right\}$ of $Y$ such that $\mathcal{E} \|_{Y_{i}}$ is ı-mixed for any $i$.

Proof. Let us show the first claim. By 2.2.1 (*), there exists a smooth stratification $\left\{Y_{i}\right\}_{1 \leq i \leq r}$ of $Y$ such that $\mathcal{F}^{\prime}\left\|_{Y_{i}}, \mathcal{F}\right\|_{Y_{i}}, \mathcal{F}^{\prime \prime} \|_{Y_{i}}$ are in $F-D_{\text {isoc }}^{\mathrm{b}}\left(Y_{i}, \mathcal{P} / K\right)$ for any $i=1, \ldots, r$. We conclude thanks to Lemma 2.1.12. Now, let us show the second statement. By Proposition 2.2.4 and Lemma 2.2.6, if $\mathcal{E}$ is a $F$-complex in $F-D_{\mathrm{m}}^{\mathrm{b}}(Y, \mathcal{P} / K)\left(\right.$ resp. $\left.F-D_{\geq w}^{\mathrm{b}}(Y, \mathcal{P} / K)\right)$ then so is $\mathbb{R} \Gamma_{Y_{i}}^{\dagger}(\mathcal{E})=j_{i+} \circ j_{i}^{!}(\mathcal{E})$ where $j_{i}=$ $(\star$, id, id, id $):\left(Y_{i}, X_{i}, \mathcal{P}, \mathcal{Q}\right) \rightarrow(Y, X, \mathcal{P}, \mathcal{Q})$ is the morphism of frames. Conversely, suppose that the $F$ complex $\mathbb{R}_{Y_{i}}^{\dagger}(\mathcal{E})$ is in $F-D_{\mathrm{m}}^{\mathrm{b}}(Y, \mathcal{P} / K)$ (resp. $\left.F-D_{\geq w}^{\mathrm{b}}(Y, \mathcal{P} / K)\right)$ for any $i=1, \ldots r$. Then the second claim follows since $\mathcal{E}$ possesses the same property by the following triangle:

$$
\mathbb{R} \underline{\Gamma}_{\sqcup_{j=i+1, \ldots, r} Y_{i}}^{\dagger}(\mathcal{E}) \rightarrow \mathbb{R} \underline{\Gamma}_{\sqcup_{j=i, \ldots, r} Y_{i}}^{\dagger}(\mathcal{E}) \rightarrow \mathbb{R} \underline{\Gamma}_{Y_{i}}^{\dagger}(\mathcal{E}) \stackrel{+}{\rightarrow},
$$

and by using the first part of the proof. By using Lemma 2.1.6.4, the first and the second claim implies the third one. 
Now, we show that the notion of $\iota$-mixedness only depends on couple $(Y, X)$ and not on the auxiliary choices.

Proposition 2.2.8. Let $* \in\{\mathrm{m}, \geq w, \leq w\}$.

1. Let $\mathbb{Y}$ be a couple and choose an l.p. frame $(Y, X, \mathcal{P}, \mathcal{Q})$ of $\mathbb{Y}$. The category $F-D_{*}^{\mathrm{b}}(Y, \mathcal{P} / K)$ does not depend on the choice of the l.p. frame of $\mathbb{Y}$. We denote by $F-D_{*}^{\mathrm{b}}(\mathbb{Y} / K)$ the corresponding category.

2. Let $Y$ be a realizable variety, and choose an l.p. frame $(Y, X, \mathcal{P}, \mathcal{Q})$ of $Y$ such that $X$ is proper. The category $F-D_{*}^{\mathrm{b}}(Y, \mathcal{P} / K)$ does not depend on the choice of the l.p. frame. We denote by $F-D_{*}^{\mathrm{b}}(Y / K)$ the corresponding category.

Proof. Let us only prove the second one, since the first part of the proposition can be checked similarly. By using fiber products, we reduce to the case where there exists a complete morphism of l.p. frames $u:\left(Y, X^{\prime}, \mathcal{P}^{\prime}, \mathcal{Q}^{\prime}\right) \rightarrow(Y, X, \mathcal{P}, \mathcal{Q})$. In this case, the proposition is a consequence of Lemma 2.2.5.

Proposition 2.2.9. Let $\mathbb{Y}$ be a couple, and $\mathcal{E}, \mathcal{E}^{\prime} \in F$ - $D_{\text {ovhol }}^{\mathrm{b}}(\mathbb{Y} / K)$. If $\mathcal{E}$ is $\iota$-mixed of weight $\geq w$ and $\mathcal{E}^{\prime}$ is $\iota$-mixed of weight $\geq w^{\prime}$ then $\mathcal{E} \widetilde{\otimes}_{\mathbb{Y}} \mathcal{E}^{\prime}$ is $\iota$-mixed of weight $\geq w+w^{\prime}$.

Proof. By paragraph 1.1 .9 (i), $\widetilde{\otimes}$ commutes with $j_{+}$where $j$ is a c-immersion. Thus, by dévissage, we may reduce to the case where $Y$ is smooth and $\mathcal{E}, \mathcal{E}^{\prime} \in F$ - $D_{\text {isoc,m }}^{\mathrm{b}}(Y, \mathcal{P} / K)$. In this isocrystal case, use paragraph 1.1.9 (i) again to show that the $\iota$-mixedness is preserved and the estimation of weights.

Lemma 2.2.10. Let $\mathcal{V} \rightarrow \mathcal{V}^{\prime}$ be a finite homomorphism of complete discrete valuation ring with mixed characteristic $(0, p)$, and $k \rightarrow k^{\prime}$ be the induced homomorphism of residue fields. Let $K^{\prime}$ be the field of fractions of $\mathcal{V}^{\prime}$, and we put $X:=X \otimes_{k} k^{\prime}, Y^{\prime}:=Y \otimes_{k} k^{\prime}$. Let $\mathcal{E} \in F-D_{\mathrm{ovhol}}^{\mathrm{b}}(Y, X / K)$ and $\mathcal{E}^{\prime}$ be the induced object of $F$ - $D_{\mathrm{ovhol}}^{\mathrm{b}}\left(Y^{\prime}, X^{\prime} / K^{\prime}\right)$. Then $\mathcal{E}$ is $\iota$-mixed (resp. of weight $\geq w$, resp. of weight $\leq w$ ) if and only if so is $\mathcal{E}^{\prime}$.

Proof. We leave the verification to the reader.

\subsection{Estimation of weights in the curve case}

In this section, we estimate the weights for curves, which is used in the proof of the main result. The proof is similar to that of Laumon in [Lau87], by using the methods developed in [AM11].

Definition 2.3.1. Let $\mathbb{Y}=(Y, X)$ be a couple such that $Y$ is smooth, $\mathcal{E}$ be an object of $F$-Isoc ${ }^{\dagger}(\mathbb{Y} / K)$, and $i_{y}:(y, y) \rightarrow \mathbb{Y}$ be the canonical morphism for a closed point $y$ of $Y$. The object $\mathcal{E}$ is said to be $\iota$-real if for any closed point $y$ of $Y$, the characteristic polynomial $\iota \operatorname{det}\left(1-t \cdot \operatorname{Frob}_{y} ; i_{y}^{+}(\mathcal{E})\right)$, which is a priori in $\mathbb{C}[t]$, is in $\mathbb{R}[t]$.

Theorem 2.3.2 ([Cre98], Theorem 10.5). If an F-isocrystal $\mathcal{E}$ on a smooth curve over $k$ is $\iota$-real, then any constituent of $\mathcal{E}$ is ı-pure.

Proof. It suffices to note that we did not assume $\mathcal{E}$ to be quasi-unipotent contrary to the statement of [Cre98, $10.5]$ since the assumption is used only to assure the finiteness of $\mathcal{E}$ and $\otimes^{2 k} \mathcal{E}$ as written in ibid., which is now known to be true if there is a Frobenius structure.

2.3.3. Let $\mathcal{X}$ be a smooth formal curve over $\mathcal{V}$, and $x$ be a closed point. For a holonomic module $\mathcal{E}$ on $\mathcal{X}$, we denote by $\Psi_{x}(\mathcal{E})$ the nearby cycle $\Psi\left(\left.\mathcal{E}\right|_{S_{x}}\right)$ using the notation of [AM11, 2.1.8] (as well as 1.5.4). The theorem below follows directly from a result of Crew.

Theorem ([Cre98], Theorem 10.8). Let $\mathcal{X}$ be a smooth formal curve over $\mathcal{V}$, s be a closed point of $\mathcal{X}$, and we put $\mathcal{U}:=\mathcal{X} \backslash\{s\}$. Let $j: \mathcal{U} \hookrightarrow \mathcal{X}$. Let $\mathcal{E}$ be an overconvergent $F$-isocrystal $\iota$-pure of weight $w$ on $\mathcal{U}$.

(i) Let $\alpha$ (resp. $\beta)$ be an eigenvalue of the Frobenius acting on $\mathcal{H}_{\mathrm{t}}^{-1}\left(i^{+} j_{+} \mathcal{E}\right)\left(\right.$ resp. $\left.\mathcal{H}_{\mathrm{t}}^{0}\left(i^{+} j_{+} \mathcal{E}\right)\right)$. Then we have the following estimations:

$$
|\iota(\alpha)| \leq q^{\operatorname{deg}(s)(w-1) / 2}, \quad|\iota(\beta)| \geq q^{\operatorname{deg}(s)(w+1) / 2}
$$


(ii) We put $\Psi:=\Psi_{s}\left(j_{+} \mathcal{E}\right)$. Assume that the action of $N$ on $\Psi / \Psi^{I=1, N=0}$ is trivial. Let $\alpha$ be the eigenvalue of the Frobenius acting on the latter quotient. Then $|\iota(\alpha)|=q^{\operatorname{deg}(s) w / 2}$.

Proof. Let $E$ be a differential $F$-module around $s$ associated to $\mathcal{E}$. The nilpotent operator $N$ on the vector space $\Psi(E)(\cong \Psi)$ induces the monodromy filtration $M$ (cf. [Del80, 1.6.1]). By Theorem 1.5.9 (i), $(\Psi(-1), M)$ coincides with $\left(\mathcal{E}_{\eta}, \mathcal{M}\right)$ using the notation of $[\mathrm{Cre} 98,10.6]$, thus, $\operatorname{gr}_{i}^{M} \Psi(-1) \cong \operatorname{gr}_{i}^{\mathcal{M}}\left(\mathcal{E}_{\eta}\right)$. The weight of $E$ is $w+1$ by Remark 2.1.4, and [Cre98, Theorem 10.8] implies that $\operatorname{gr}_{i}^{M} \Psi(-1)$ is purely of weight $w+1+i$. Thus, $\Psi^{I=1, N=0}(-1) \subset \Psi^{N=0}(-1) \subset M_{0}(\Psi)(-1)$ is $\iota$-weight $\leq w+1$. We get (i) by applying Theorem 1.5.9 (iii) and duality. Under the situation of (ii), $\Psi / \Psi^{I=1, N=0}$ is nothing but $\operatorname{gr}_{1}^{M}(\Psi)$, and we get what we wanted.

2.3.4. Let us briefly review the geometric Fourier transform [NH04], only in the affine space case.

Fix $\pi \in \overline{\mathbb{Q}}_{p}$ such that $\pi^{p-1}=-p$. We assume that $\pi \in K$. We denote by $\mathcal{L}_{\pi}$ the Artin-Schreier isocrystal in $F$-Isoc ${ }^{\dagger \dagger}\left(\mathbb{A}_{k}^{1} / K\right)$. Since $\mathcal{L}_{\pi}$ is a direct factor of $\operatorname{Art}_{*} \mathcal{O}_{\mathbb{A}_{k}^{1}}$ (cf. [Ber84, 1.5]), where Art: $\mathbb{A}_{k}^{1} \rightarrow \mathbb{A}_{k}^{1}$ is the Artin-Schreier morphism, the weight of $\mathcal{L}_{\pi}[1]$ is 0 since the weight is preserved under push-forward by finite étale morphism.

Now, consider the following diagram: $\left(\mathbb{A}_{k}^{n}\right)^{\prime} \stackrel{p_{2}}{\longleftarrow} \mathbb{A}_{k}^{2 n} \stackrel{p_{1}}{\longrightarrow} \mathbb{A}_{k}^{n}$, where $\left(\mathbb{A}_{k}^{n}\right)^{\prime}$ is the "dual affine space", which is nothing but $\mathbb{A}_{k}^{n}$. We denote by $\delta: \mathbb{A}_{k}^{n} \times\left(\mathbb{A}_{k}^{n}\right)^{\prime} \rightarrow \mathbb{A}_{k}^{1}$ the canonical duality bracket induced by $t \mapsto \sum_{i=1}^{n} x_{i} y_{i}$. We put ${ }^{(8)} \mathcal{K}_{\pi}:=\delta^{!}\left(\mathcal{L}_{\pi}[-1]\right)$. For any $\mathcal{E} \in F-D_{\text {ovhol }}^{\mathrm{b}}\left(\mathbb{A}_{k}^{n}\right)$, the geometric Fourier transform $\mathcal{F}_{\pi}(\mathcal{E})$ is defined to be $p_{2+}\left(p_{1}^{!} \mathcal{E}_{\mathbb{\otimes}^{2 n}} \mathcal{K}_{\pi}\right)$ (cf. $\left.[\mathrm{NH} 04,3.2 .1]^{(9)}\right)$. Important properties for us are that:

1. if $\mathcal{E}$ is a $F$-module, $\mathcal{F}_{\pi}(\mathcal{E})[2-n]$ is a $F$-module as well (cf. [NH04, Theorem 5.3.1]).

2. $\mathcal{F}_{\pi}^{\prime} \circ \mathcal{F}_{\pi}(\mathcal{E})[4-2 n] \cong \mathcal{E}(n)$ where $\mathcal{F}_{\pi}^{\prime}$ denotes the geometric Fourier transform for dual affine space (cf. [AM11, Remark 3.2.8]).

2.3.5. In this subsection, we use the Fourier transform for $n=1$. Let $\mathbb{A}:=\mathbb{A}^{1}$. As in $[$ Lau 87, 1.4.2], consider the following three types of irreducible overholonomic $F$-modules $\mathcal{E}$ on $\mathbb{A}$ :

(T1) Let $s$ be a closed point of $\mathbb{A}$, and $i_{s}: s \hookrightarrow \mathbb{A}$. Then $\mathcal{E} \cong i_{s+} V$ where $V$ is an irreducible $K$-vector space with Frobenius.

(T2) There exists a simple overholonomic $F$-module $\mathcal{E}^{\prime}$ on $\mathbb{A}^{\prime}$ of type $(\mathrm{T} 1)$ such that $\mathcal{E} \cong \mathcal{F}_{\pi}\left(\mathcal{E}^{\prime}\right)$.

(T3) We have $\mathcal{E} \cong j_{!_{+}} \mathcal{F}$ where $j: \mathbb{A} \backslash S \hookrightarrow \mathbb{A}$ with $S$ a finite subset of $\mathbb{A}$ and $\mathcal{F}$ is an irreducible $F$-isocrystal which is not of type (T2).

These F-modules are irreducible, since Fourier transform is involutive by 2.3.4.2 and by Proposition 1.4.7. Moreover, irreducible objects can be classified in the above three types by Theorem 1.4.9 (i). By definition, $F$-modules of type (T1) are transformed under Fourier transform to (T2) and (T2) to (T1). Thus $F$-modules of type (T3) are transformed under Fourier transform to (T3).

An $F$-module $\mathcal{E}$ is said to be geometrically constant if there exists an isomorphism $\mathcal{E} \cong \mathcal{O}_{\mathbb{A}}^{\oplus r}$ for some $r$ as $\mathcal{D}$-modules without Frobenius structure. We may check that $\mathcal{F}_{\pi}\left(i_{0+}(V)\right)$ is geometrically constant. Moreover, for $s \neq 0, \mathcal{F}_{\pi}\left(i_{s+}(V)\right)$ is ramified at infinity. In other words, an $F$-isocrystal $\mathcal{E}$ on $\mathbb{A}$ which is not geometrically constant and unramified at $\infty$ (i.e. is $\mathcal{O}$-coherent around $\infty$ ) is of type (T3).

Lemma 2.3.6. Let $p: \mathbb{A}:=\mathbb{A}^{1} \rightarrow \operatorname{Spec}(k)$ be the structural morphism, $S$ be a set of closed points in $\mathbb{A}$, and $j: U:=\mathbb{A} \backslash S \hookrightarrow \mathbb{A}$ be the canonical inclusion. Let $\mathcal{E}$ be an overconvergent $F$-isocrystal purely of $\iota$-weight $w$ on $U$. We assume moreover that $\mathcal{E}$ is irreducible, unramified at $\infty$, and not geometrically constant. Then for any eigenvalue $\alpha$ of the Frobenius acting on $H^{0}\left(p_{+} j_{+} \mathcal{E}\right)$, we have the estimation $|\iota(\alpha)| \geq q^{w / 2}$.

(8) There is a typo in the definition of $K_{\pi}$ in [NH04, 3.1.1]: the shift of $K_{\pi}$ is not $[2-2 N]$ but $[2 N-2]$.

${ }^{(9)}$ Notice that our twisted tensor product and hers are the same. 
Proof. Since we may change $K$ by its totally ramified extension by Lemma 2.2.10, we may assume and fix a root $\pi$ of the equation $x^{p-1}+p=0$ in $K$. By [AM11, Proposition 4.1.6 (ii)], $0^{\prime} \in \mathbb{A}^{\prime}$ is the only singularity of $\mathcal{F}_{\pi}(\mathcal{E})$. By assumption, $\mathcal{E}$ is a simple $F$-module of type $(\mathrm{T} 3)$. This is showing that $\mathcal{F}_{\pi}(\mathcal{E})$ is of type (T3) as well, and thus there exists an irreducible overconvergent $F$-isocrystal $\mathcal{E}^{\prime}$ on $\mathbb{A}^{\prime} \backslash\left\{0^{\prime}\right\}$ such that $\mathcal{F}_{\pi}\left(j_{!+} \mathcal{E}[1]\right) \cong j_{!_{+}}^{\prime}\left(\mathcal{E}^{\prime}\right)$ where $j^{\prime}: \mathbb{A}^{\prime} \backslash\left\{0^{\prime}\right\} \hookrightarrow \mathbb{A}^{\prime}$. By duality, it suffices to show that any eigenvalue of $\mathcal{H}_{\mathrm{t}}^{0}\left(p_{!} j_{!} \mathcal{E}\right)$ is of weight $\leq w$, and by Theorem 2.3.3 (i), we are reduced to showing that $\mathcal{H}_{\mathrm{t}}^{0}\left(p_{!} j_{!+} \mathcal{E}\right)$ is of weight $\leq w$.

In this proof, for the notation being compatible with [AM11], we introduce the following: for a smooth curve $q: X \rightarrow \operatorname{Spec}\left(k^{\prime}\right)$ where $k^{\prime}$ is a field over $k$ and a overholonomic $F$-module $\mathcal{F}$ on $X$, we denote $\mathcal{H}_{\mathrm{t}}^{i}(q ! \mathcal{F})$ by $H_{\mathrm{rig}, c}^{i+1}(X, \mathcal{F})(1)$. Using this notation, we need to show that $H_{\mathrm{rig}, c}^{1}\left(\mathbb{A}, j_{!_{+}} \mathcal{E}\right)(1)$ is of weight $\leq w$. By the same argument as [AM11, 6.2.8] (here we need the assumption that this is unramified at $\infty$ ), we have an exact sequence of Deligne modules

$$
0 \rightarrow H_{\mathrm{rig}, c}^{1}\left(\mathbb{A}_{\bar{k}}, j_{!+} \mathcal{E}\right) \rightarrow \Psi_{0^{\prime}}\left(\left.\left(j_{!+} \mathcal{E}\right)^{\wedge}\right|_{S_{0^{\prime}}}\right)(-2) \rightarrow\left(\left.K^{\mathrm{ur}} \otimes_{K} \mathcal{E}\right|_{s_{\infty}}\right)(-1) \rightarrow \underbrace{H_{\mathrm{rig}, c}^{2}\left(\mathbb{A}_{\bar{k}}, j j_{!+} \mathcal{E}\right)}_{=0} \rightarrow 0 .
$$

Moreover, we have

$$
\left(\Psi_{0^{\prime}}\left(\left(j_{!+} \mathcal{E}\right)^{\wedge}\right)(-2)\right)^{I=1, N=0} \cong \mathcal{H}_{\mathrm{t}}^{-1}\left(i_{0^{\prime}}^{+} j_{!+}^{\prime}\left(\mathcal{E}^{\prime}(-2)\right)\right) \cong \mathcal{H}_{\mathrm{t}}^{0}\left(p_{!} j_{!+} \mathcal{E}(-1)\right) \cong H_{\mathrm{rig}, c}^{1}\left(\mathbb{A}_{\bar{k}}, j_{!+} \mathcal{E}\right)
$$

where $I$ denotes the inertia group at $0^{\prime}$. The first isomorphism is deduced by Theorem 1.5.9 (iii).

Now, assume $\mathcal{E}^{\prime}$ is ı-pure of weight $w^{\prime} \in \mathbb{R}$. Then Theorem 2.3 .3 (ii) can be applied to get

$$
\Psi_{0^{\prime}}\left(\mathcal{E}^{\prime}\right)(-2) /\left(\Psi_{0^{\prime}}\left(\mathcal{E}^{\prime}\right)(-2)\right)^{I=1, N=0} \cong\left(\left.K^{\mathrm{ur}} \otimes_{K} \mathcal{E}\right|_{s_{\infty}}\right)(-1)
$$

is $\iota$-pure of weight $w^{\prime}+4$. Applying Theorem 2.3 .3 (i) to $\mathcal{E}$ and $\mathbb{D}(\mathcal{E}),\left(\left.K^{\mathrm{ur}} \otimes_{K} \mathcal{E}\right|_{s_{\infty}}\right)$ is $\iota$-pure of $\iota$-weight $w+1$, which implies that $w^{\prime}=w-1$. Applying Theorem 2.3.3 (i) to the isomorphisms ( $\star$ ), we get the desired upper bound.

It remains to show that $\mathcal{E}^{\prime}$ is $\iota$-pure. Using Theorem 2.3.2, it suffices to find an $\iota$-real overconvergent $F$-isocrystal $\mathcal{G}^{\prime}$ on $\mathbb{A}^{\prime} \backslash\left\{0^{\prime}\right\}$ of which $\mathcal{E}^{\prime}$ is a constituent. For this, we just follow exactly the same line as the last half part of [Lau87, (4.4)], and we omit the detail here.

Theorem 2.3.7. Let $f: X \rightarrow \operatorname{Spec}(k)$ be a smooth curve, and $\mathcal{E}$ be an object of $F-D_{\geq w}^{\mathrm{b}}(X / K)$. Then $f_{+} \mathcal{E}$ is of weight $\geq w$.

Proof. By dévissage, we may assume $\mathcal{E}$ to be an overconvergent $F$-isocrystal on $X$. Let $Z$ be a closed subscheme of $X$. By Proposition 2.2.7, $\mathbb{R} \underline{\Gamma}_{Z}^{\dagger}(\mathcal{E})$ is $\iota$-mixed of weight $\geq w$. Thus by considering the localization triangle, we may replace $X$ by $X \backslash Z$, and shrink $X$. Since, by shrinking $X$, there exists a non-constant morphism $g: X \stackrel{g}{\rightarrow} X^{\prime} \subset \mathbb{P}_{k}^{1}$ such that $g$ is finite étale, we may assume that $X \subset \mathbb{P}^{1}$. Since it suffices to show the theorem after taking a finite extension of $k$, we may assume that $\infty \in \mathbb{P}^{1}$ is contained in $X$. Let $\widetilde{\mathcal{E}}$ be the push-forward of $\mathcal{E}$ to $\mathbb{P}^{1}$. We put $j: \mathbb{A}^{1} \hookrightarrow \mathbb{P}^{1}$ and $i:\{\infty\} \hookrightarrow \mathbb{P}^{1}$. Consider the following exact sequence:

$$
\mathbb{R} \underline{\Gamma}_{\{\infty\}}^{\dagger}(\mathcal{E}) \rightarrow \widetilde{\mathcal{E}} \rightarrow j_{+}\left(\widetilde{\mathcal{E}} \|_{\mathbb{A}^{1}}\right) \stackrel{+1}{\longrightarrow} .
$$

Since $\mathbb{R} \underline{\Gamma}_{\{\infty\}}^{\dagger}(\mathcal{E})$ is $\iota$-mixed of weight $\geq w$, it is reduced to showing that $p_{+}\left(\widetilde{\mathcal{E}} \|_{\mathbb{A}^{1}}\right)$ is of weight $\geq w$ where $p: \mathbb{A}^{1} \rightarrow \operatorname{Spec}(k)$ is the structural morphism. We may assume $\mathcal{E}$ to be irreducible. When $\mathcal{E}$ is not geometrically constant, then the theorem follows by Lemma 2.3.6. Otherwise, the verification is easy.

Remark. In fact, this theorem is not completely new, and by Remark 2.1.4, it follows directly from the main theorem of [Ked06]. However, the proof is independent.

\section{Monodromy filtration of a convergent log-isocrystal}

Throughout this section, we consider situation (B) in Notation and convention. Before starting the section, let us fix notation. 


\subsection{Notation}

Until §3.5, we consider the following situation unless otherwise stated.

3.1.1. Let $\phi: \mathcal{X} \rightarrow \widehat{\mathbb{A}}_{\mathcal{V}}^{d}$ be an étale morphism of affine formal $\mathcal{V}$-schemes and let $t_{1}, \ldots, t_{d}$ be the corresponding local coordinates, i.e. the image of the coordinates $x_{1}, \ldots, x_{d}$ under the homomorphism of complete $\mathcal{V}$ algebras $\mathcal{V}\left\{x_{1}, \ldots, x_{d}\right\} \rightarrow \Gamma\left(\mathcal{X}, \mathcal{O}_{\mathcal{X}}\right)$ given by $\phi$. For any $i=1, \ldots, d$, we put $\mathcal{Z}_{i}=V\left(t_{i}\right)$. We denote by $A:=\Gamma\left(\mathcal{X}, \mathcal{O}_{\mathcal{X}}\right), B:=A / t_{1} A$, so $\mathcal{Z}_{1}:=\operatorname{Spf} B$. Fix $1 \leq r \leq d$, and we denote by $\mathfrak{D}$ the strict normal crossing divisor of $\mathcal{X}$ whose irreducible components are that of $\mathcal{Z}_{2} \cup \cdots \cup \mathcal{Z}_{r}$ and $\mathfrak{D}=\emptyset$ if $r=1$. Put $\mathcal{Z}:=\mathcal{Z}_{1} \cup \mathfrak{D}$. We get a strict normal crossing divisor of $\mathcal{Z}_{1}$ defined by $\mathfrak{D}_{1}:=\bigcup_{i=2}^{r} \mathcal{Z}_{1} \cap \mathcal{Z}_{i}$. We put $\mathcal{Y}:=\mathcal{X} \backslash \mathcal{Z}$, and let $j: \mathcal{Y} \subset \mathcal{X}$ be the open immersion, $u:(\mathcal{X}, \mathcal{Z}) \rightarrow \mathcal{X}, u_{1}:(\mathcal{X}, \mathcal{Z}) \rightarrow(\mathcal{X}, \mathfrak{D}), v:(\mathcal{X}, \mathfrak{D}) \rightarrow \mathcal{X}, i_{1}: \mathcal{Z}_{1} \hookrightarrow \mathcal{X}$, $i_{1}^{\#}:\left(\mathcal{Z}_{1}, \mathfrak{D}_{1}\right) \rightarrow(\mathcal{X}, \mathfrak{D}), w_{1}:\left(\mathcal{Z}_{1}, \mathfrak{D}_{1}\right) \rightarrow \mathcal{Z}_{1}$ be the canonical morphisms of $\log$ schemes. We sometimes denote $\mathcal{X}^{b}:=(\mathcal{X}, \mathcal{Z}), \mathcal{X}^{\#}:=(\mathcal{X}, \mathfrak{D}), \mathcal{Z}_{1}^{\#}:=\left(\mathcal{Z}_{1}, \mathfrak{D}_{1}\right)$ for simplicity.

3.1.2. Let $\bar{t}_{2}, \ldots, \bar{t}_{d}$ be the global sections of $\mathcal{O}_{\mathcal{Z}_{1}}$ induced by $t_{2}, \ldots, t_{d}$ and let $\phi_{1}: \mathcal{Z}_{1} \rightarrow \widehat{\mathbb{A}}_{\mathcal{V}}^{d-1}$ be the étale morphism induced by the local coordinates $\bar{t}_{2}, \ldots, \bar{t}_{d}$. The homomorphism of $\mathcal{V}$-algebras $\mathcal{V}\left\{x_{1}, \ldots, x_{d}\right\} \rightarrow$ $B\left\{x_{1}\right\}$ given by $x_{1} \mapsto x_{1}$ and $x_{i} \mapsto \bar{t}_{i}$ for $i \geq 2$ induces the morphism of smooth formal $\mathcal{V}$-schemes $i d \times$ $\phi_{1}: \widehat{\mathbb{A}}_{\mathcal{V}}^{1} \times \mathcal{Z}_{1} \rightarrow \widehat{\mathbb{A}}_{\mathcal{V}}^{d}$. The homomorphism of $B$-algebras $B\left\{x_{1}\right\} \rightarrow B$ given by $x_{1} \mapsto 0$ gives a closed immersion of smooth formal $\mathcal{V}$-schemes denoted by $i_{1}^{\prime \prime}: \mathcal{Z}_{1} \hookrightarrow \widehat{\mathbb{A}}_{\mathcal{V}}^{1} \times \mathcal{Z}_{1}$. By putting $\mathcal{X}^{\prime}:=\left(\widehat{\mathbb{A}}^{1} \times \mathcal{Z}_{1}\right) \times \widehat{\mathbb{A}}^{n} \mathcal{X}$, we get the closed immersion $i_{1}^{\prime}: \mathcal{Z}_{1} \hookrightarrow \mathcal{X}^{\prime}$ making commutative the diagram

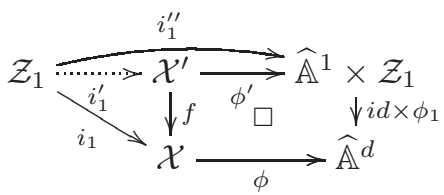

where the square is cartesian with etale morphisms and where $f$ and $\phi^{\prime}$ are the canonical projections. Putting $\mathcal{Z}_{1}^{\prime}:=f^{-1} \mathcal{Z}_{1}=\mathcal{Z}_{1} \times \mathcal{X} \mathcal{X}^{\prime}$, we get a section $\left(i d, i_{1}^{\prime}\right): \mathcal{Z}_{1} \hookrightarrow \mathcal{Z}_{1}^{\prime}$ of the etale projection $\mathcal{Z}_{1}^{\prime} \rightarrow \mathcal{Z}_{1}$. Hence, by using SGA1 Exp. I Corollary 5.3. , by shrinking $\mathcal{X}^{\prime}$ but not $\mathcal{X}$, we may assume that $\mathcal{Z}_{1} \stackrel{\sim}{\rightarrow} \mathcal{Z}_{1} \times \mathcal{X} \mathcal{X}^{\prime}$. Let $g_{1}: \mathcal{X}^{\prime} \rightarrow \mathcal{Z}_{1}$ be the canonical morphism, i.e. the composition of $\phi^{\prime}$ and the projection $\widehat{\mathbb{A}}^{1} \times \mathcal{Z}_{1} \rightarrow \mathcal{Z}_{1}$. By construction, $i_{1}^{\prime}$ is a section of $g_{1}$.

We put $\mathfrak{D}^{\prime}:=f^{-1}(\mathfrak{D}), \mathcal{Z}^{\prime}:=f^{-1}(\mathcal{Z}), \mathcal{X}^{\prime b}:=\left(\mathcal{X}^{\prime}, \mathcal{Z}^{\prime}\right), \mathcal{X}^{\prime \#}:=\left(\mathcal{X}^{\prime}, \mathfrak{D}^{\prime}\right)$. We denote by $f^{\#}: \mathcal{X}^{\prime \#} \rightarrow \mathcal{X}^{\#}$, $f^{b}: \mathcal{X}^{\prime b} \rightarrow \mathcal{X}^{b}, u_{1}^{\prime}: \mathcal{X}^{\prime b} \rightarrow \mathcal{X}^{\prime \#}, g_{1}^{b}: \mathcal{X}^{\prime b} \rightarrow \mathcal{Z}_{1}^{\#}, g_{1}^{\#}: \mathcal{X}^{\prime \#} \rightarrow \mathcal{Z}_{1}^{\#}, i_{1}^{\prime \#}: \mathcal{Z}_{1}^{\#} \rightarrow \mathcal{X}^{\prime \#}, v^{\prime}: \mathcal{X}^{\prime \#} \rightarrow \mathcal{X}^{\prime}$ the associated morphisms. We summarize the notation and the above properties in the following commutative diagrams:
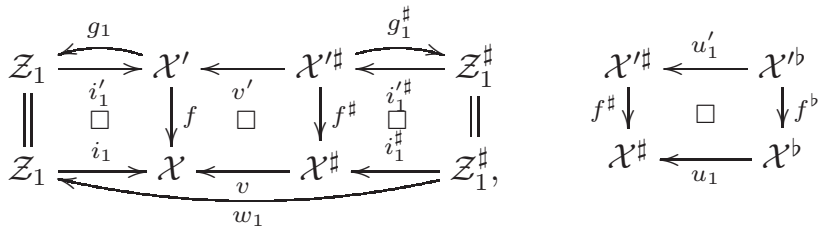

where squares (including the below "square" containing $w_{1}$ ) are cartesian, where the commutativity above means that $i_{1}^{\prime}$ is a section of $g_{1}$ and $i_{1}^{\prime \#}$ is a section of $g_{1}^{\#}$.

We note that $\mathfrak{D}^{\prime}=g_{1}^{-1}\left(\mathfrak{D}_{1}\right)$. Indeed, since $\mathfrak{D}=\phi^{-1}\left(V\left(x_{2} \ldots x_{d}\right)\right)$, then using the commutativity of the square $(3.1 .2 .1)$ we get $\mathfrak{D}^{\prime}=f^{-1} \phi^{-1}\left(V\left(x_{2} \ldots x_{d}\right)\right)=\phi^{\prime-1}\left(i d \times \phi_{1}\right)^{-1}\left(V\left(x_{2} \ldots x_{d}\right)\right)=\phi^{\prime-1}\left(\widehat{\mathbb{A}}^{1} \times \mathfrak{D}_{1}\right)=$ $g_{1}^{-1}\left(\mathfrak{D}_{1}\right)$. Moreover, since $i_{1}^{\prime}$ is a section of $g_{1}$, then this implies $\mathfrak{D}^{\prime}=g_{1}^{-1} \circ i_{1}^{\prime-1}\left(\mathfrak{D}^{\prime}\right)$.

3.1.3 (Canonical Frobenius). Let $F_{\widehat{\mathbb{A}}_{\mathcal{V}}^{d}}: \widehat{\mathbb{A}}_{\mathcal{V}}^{d} \rightarrow \widehat{\mathbb{A}}_{\mathcal{V}}^{d}$ and $F_{\widehat{\mathbb{A}}_{\mathcal{V}}^{d-1}}: \widehat{\mathbb{A}}_{\mathcal{V}}^{d-1} \rightarrow \widehat{\mathbb{A}}_{\mathcal{V}}^{d-1}$ be the canonical (s-th) Frobenius endomorphisms given by the formula $P(\underline{x}) \mapsto P\left(\underline{x}^{q}\right)$ for $\underline{x}=\left(x_{1}, \ldots, x_{d}\right)$ or $\underline{x}=\left(x_{1}, \ldots, x_{d-1}\right)$ respectively. Since the morphism $\phi: \mathcal{X} \rightarrow \widehat{\mathbb{A}}_{\mathcal{V}}^{d}$ (resp. $\phi_{1}: \mathcal{Z}_{1} \rightarrow \widehat{\mathbb{A}}_{\mathcal{V}}^{d-1}$ ) is étale, the $s$ th Frobenius endomorphism of $X$ (resp. of $Z_{1}$ ) has a unique lifting $F_{\mathcal{X}}: \mathcal{X} \rightarrow \mathcal{X}$, (resp. $F_{\mathcal{Z}_{1}}: \mathcal{Z}_{1} \rightarrow \mathcal{Z}_{1}$ ) which commutes with the canonical Frobenius 
endomorphism of $\widehat{\mathbb{A}}_{\mathcal{V}}^{d}$ (resp. $\widehat{\mathbb{A}}_{\mathcal{V}}^{d-1}$ ). From $F_{\mathcal{X}}$ and $F_{\mathcal{Z}_{1}}$ we get canonically the factorisations $F_{\mathcal{X}^{b}}: \mathcal{X}^{b} \rightarrow \mathcal{X}^{b}$ $F_{\mathcal{Z}_{1}^{\#}}: \mathcal{Z}_{1}^{\#} \rightarrow \mathcal{Z}_{1}^{\#}$. Since the local coordinates $t_{1}, \ldots, t_{d}$ are fixed we can call abusively these Frobenius endomorphisms "canonical".

3.1.4 (Log- $\nabla$-modules, logarithmic arithmetic $\mathcal{D}$-modules and convergent log isocrystals). We recall here the following facts. We denote by $\mathcal{D}_{\mathcal{X}^{b}}$ the usual sheaf of differential operators of $\mathcal{X}^{b} / \operatorname{Spf} \mathcal{V}$. Let $\mathcal{D}_{\mathcal{X}^{b}}^{\dagger}$ be the sheaf of differential operators of finite level and infinite order on the formal log-scheme $\mathcal{X}^{b}$, which is a kind of weak completion of $\mathcal{D}_{\mathcal{X}^{b}}$ as $\mathcal{O}_{\mathcal{X}}$-ring (e.g. see [Car09a, 4.4] for the explicit construction). We denote by sp: $\mathcal{X}_{K} \rightarrow \mathcal{X}$ the specialisation morphism from $\mathcal{X}_{K}$, the rigid analytic space of $\mathcal{X}$, to $\mathcal{X}$.

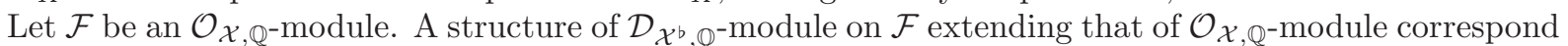
bijectively to an integrable $\log$ connection $\mathcal{F} \rightarrow \mathcal{F} \otimes_{\mathcal{O}_{\mathcal{X}}} \Omega_{\mathcal{X}^{b} / \text { Spf } \mathcal{V}}^{1}$. Hence, similarly than in [Ber96b, 4.4.2], since $\mathcal{X}$ is affine (otherwise we have to replace "projective" by "locally projective"), we check that the functors $\mathrm{sp}_{*}$ and $\mathrm{sp}^{*}$ induce quasi-inverse equivalences between the category of $\mathcal{D}_{\mathcal{X}^{b}, \mathbb{Q}^{-m o d u l e s}}$ which are projective of finite type over $\mathcal{O}_{\mathcal{X}, \mathbb{Q}}$ and the category of $\log -\nabla$-modules on $\mathcal{X}_{K}$ with respect to $t_{1}, \ldots, t_{r} \in \Gamma\left(\mathcal{X}_{K}, \mathcal{O}_{\mathcal{X}_{K}}\right)$ (relative to $\operatorname{Max}(K))$, where the last category is defined in [Ked07, 2.3.7]. On the last category, Kedlaya defined in [Ked07, 2.3.9] the notion of residue of $\nabla$ along $V\left(t_{i}\right)$, which can be translated similarly for a

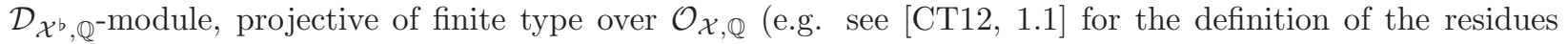
or read the beginning of 3.2.11). From [Ked07, 3.2.14], the category of log- $\nabla$-modules on $\mathcal{X}_{K}$ with respect to $t_{1}, \ldots, t_{r} \in \Gamma\left(\mathcal{X}_{K}, \mathcal{O}_{\mathcal{X}_{K}}\right)$ with nilpotent residues is abelian. Then so is the category of $\mathcal{D}_{\mathcal{X}^{b}, \mathbb{Q}^{-m o d u l e s}}$ projective of finite type over $\mathcal{O}_{\mathcal{X}, \mathbb{Q}}$ and with nilpotent residues. Kedlaya defined in [Ked07, 6.3.1], the notion of a convergent $\log -\nabla$-modules on $\mathcal{X}_{K}$ with respect to $t_{1}, \ldots, t_{r} \in \Gamma\left(\mathcal{X}_{K}, \mathcal{O}_{\mathcal{X}_{K}}\right)$ with nilpotent residues.

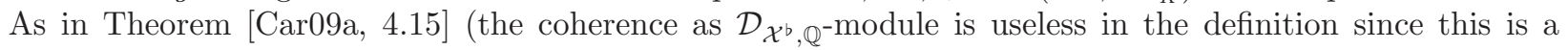

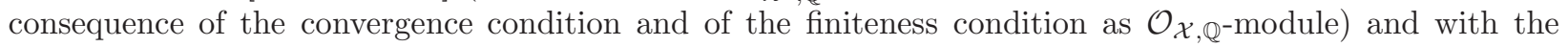

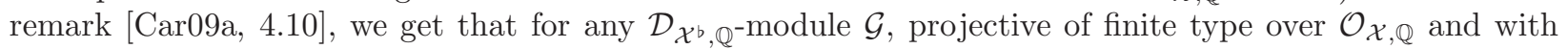
nilpotent residues, $\mathrm{sp}^{*}(\mathcal{G})$ is convergent if and only if its structure of $\mathcal{D}_{\mathcal{X}^{b}, \mathbb{Q}^{-} \text {-module extends to a structure }}$ of coherent $\mathcal{D}_{\mathcal{X}^{b}, \mathbb{Q}^{-m o d u l e . ~}}^{\dagger}$

To sum up, the functors $\mathrm{sp}_{*}$ and $\mathrm{sp}^{*}$ induce quasi-inverse equivalences from the category of coherent $\mathcal{D}_{\mathcal{X}^{b}, \mathbb{Q}^{\dagger}}^{\dagger}$-modules, projective of finite type over $\mathcal{O}_{\mathcal{X}, \mathbb{Q}}$ and with nilpotent residues to the category of convergent log- $\nabla$-modules on $\mathcal{X}_{K}$ with respect to $t_{1}, \ldots, t_{r} \in \Gamma\left(\mathcal{X}_{K}, \mathcal{O}_{\mathcal{X}_{K}}\right)$ with nilpotent residues. Since $\mathcal{D}_{\mathcal{X}^{b}, \mathbb{Q}^{-}}$ coherence is stable under kernels and cokernels, these categories are abelian subcategories of the abelian category of log- $\nabla$-modules on $\mathcal{X}_{K}$ with respect to $t_{1}, \ldots, t_{r} \in \Gamma\left(\mathcal{X}_{K}, \mathcal{O}_{\mathcal{X}_{K}}\right)$ with nilpotent residues.

We denote by $\operatorname{Isoc}\left(\mathcal{X}^{b} / K\right)$ the category of coherent $\mathcal{D}_{\mathcal{X}^{b}, \mathbb{Q}^{\dagger}}^{\dagger}$-modules, projective of finite type over $\mathcal{O}_{\mathcal{X}, \mathbb{Q}} \cdot$ As in [Car09a, 4.9], we call these objects convergent log-isocrystal on $\mathcal{X}^{b} / K$. We remark that this category is not abelian (e.g. consider the cokernel of the morphism $\alpha_{1, \mathcal{F}}$ of the paragraph 3.4.5).

We denote by $\operatorname{Isoc}^{0}\left(\mathcal{X}^{b} / K\right)$ the subcategory of $\operatorname{Isoc}\left(\mathcal{X}^{b} / K\right)$ whose objects have with nilpotent residues. The category $\operatorname{Isoc}^{0}\left(\mathcal{X}^{b} / K\right)$ is abelian.

3.1.5 (Overconvergent and convergent $F$-isocrystals). By functoriality, $\mathcal{D}_{\mathcal{X}^{b}} \underset{F_{\mathcal{X}^{b}}}{\longrightarrow} \mathcal{X}^{b}:=F_{\mathcal{X}^{*}} \mathcal{D}_{\mathcal{X}^{b}}$ is a $\left(\mathcal{D}_{\mathcal{X}^{b}}, \mathcal{D}_{\mathcal{X}^{b}}\right)-$ bimodule, where $F_{\mathcal{X}}^{*}: F_{\mathcal{X}}^{-1} \mathcal{O}_{\mathcal{X}} \rightarrow \mathcal{O}_{\mathcal{X}}$ is the Frobenius extension induced by $F_{\mathcal{X}}: \mathcal{X} \rightarrow \mathcal{X}$. We get the functor

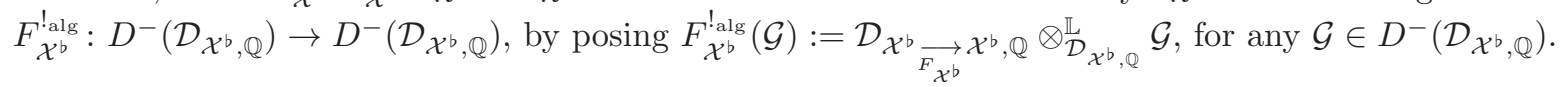

Similarly, replacing $\mathcal{D}$ by $\mathcal{D}^{\dagger}$, we get the functor $F_{\mathcal{X}^{b}}^{!}: D_{\text {coh }}^{\mathrm{b}}\left(\mathcal{D}_{\mathcal{X}^{\mathrm{b}}, \mathbb{Q}}^{\dagger}\right) \rightarrow D_{\text {coh }}^{\mathrm{b}}\left(\mathcal{D}_{\mathcal{X}^{\mathrm{b}}, \mathbb{Q}}^{\dagger}\right)$, by posing $F_{\mathcal{X}^{b}}^{!}(\mathcal{G}):=$ $\mathcal{D}_{\mathcal{X}^{b} \underset{F_{\mathcal{X}^{b}}}{\dagger} \mathcal{X}^{b}, \mathbb{Q}} \otimes_{\mathcal{D}_{\mathcal{X}^{b}, \mathbb{Q}}^{\dagger}}^{\mathbb{L}} \mathcal{G}$, for any $\mathcal{G} \in D_{\text {coh }}^{\mathrm{b}}\left(\mathcal{D}_{\mathcal{X}^{b}, \mathbb{Q}}^{\dagger}\right)$.

For any $\mathcal{G} \in \operatorname{Isoc}\left(\mathcal{X}^{b} / K\right)$, since $\mathcal{D}_{\mathcal{X}^{b}, \mathbb{Q}}^{\dagger} \otimes_{\mathcal{D}_{\mathcal{X}^{b}, \mathbb{Q}}} \mathcal{G} \stackrel{\sim}{\longrightarrow} \mathcal{G}$ (see [Car09a, 4.14]), by flatness and preservation of the notion of Kedlaya's convergence we check that $F_{\mathcal{X}^{b}}^{! \text {alg }}(\mathcal{G}) \stackrel{\sim}{\longrightarrow} F_{\mathcal{X}^{b}}^{!}(\mathcal{G}) \stackrel{\sim}{\rightarrow} F_{\mathcal{X}}^{*}(\mathcal{G})$, where the last isomorphism

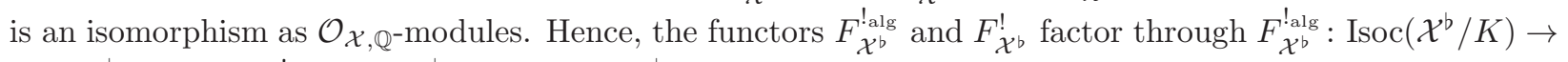
$\operatorname{Isoc}\left(\mathcal{X}^{b} / K\right)$ and $F_{\mathcal{X}^{b}}^{!}: \operatorname{Isoc}\left(\mathcal{X}^{b} / K\right) \rightarrow \operatorname{Isoc}\left(\mathcal{X}^{b} / K\right)$, which we will simply denote by $F_{\mathcal{X}}^{*}$. 


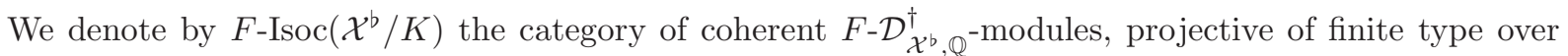
$\mathcal{O}_{\mathcal{X}, \mathbb{Q}}$. In other words, an object of $F$-Isoc $\left(\mathcal{X}^{b} / K\right)$ is the data of an object $\mathcal{F}$ of $\operatorname{Isoc}\left(\mathcal{X}^{b} / K\right)$ and an isomorphism $\phi_{\mathcal{F}}: F_{\mathcal{X}}^{*} \mathcal{F} \stackrel{\sim}{\rightarrow} \mathcal{F}$ in $\operatorname{Isoc}\left(\mathcal{X}^{b} / K\right)$. For simplicity, if there is no risk of confusion, we denote $\left(\mathcal{F}, \phi_{\mathcal{F}}\right)$ by $\mathcal{F}$. The Frobenius structure implies the nilpotence of the residues. This yields that $F$-Isoc $\left(\mathcal{X}^{b} / K\right)$ is also an abelian category. Hence, we get the forgetful functor of abelian categories $\omega: F$-Isoc $\left(\mathcal{X}^{b} / K\right) \rightarrow \operatorname{Isoc}^{0}\left(\mathcal{X}^{b} / K\right)$.

We denote by $(F-) \operatorname{Isoc}^{\dagger \dagger}(\mathcal{X}, Z / K)$ the abelian category of coherent $(F-) \mathcal{D}_{\mathcal{X}}^{\dagger}\left({ }^{\dagger} Z\right)_{\mathbb{Q}^{-} \text {-modules, }} \mathcal{O}_{\mathcal{X}}\left({ }^{\dagger} Z\right)_{\mathbb{Q}^{-}}$ coherent. In other words, an object of $(F$ - $) \operatorname{Isoc}^{\dagger \dagger}(\mathcal{X}, Z / K)$ is the data of a coherent $\mathcal{D}_{\mathcal{X}}^{\dagger}\left({ }^{\dagger} Z\right) \mathbb{Q}_{\text {-module }} \mathcal{E}$ coherent over $\mathcal{O}_{\mathcal{X}}\left({ }^{\dagger} Z\right)_{\mathbb{Q}}$ (resp. and an isomorphism $\phi_{\mathcal{E}}: F_{\mathcal{X}}^{*} \mathcal{E} \stackrel{\sim}{\rightarrow} \mathcal{E}$ of $\mathcal{D}_{\mathcal{X}}^{\dagger}\left({ }^{\dagger} Z\right)_{\mathbb{Q}}$-modules). Berthelot proved that $\mathrm{sp}_{*}$ induces an equivalence from the category of $(F$-)isocrystal on $Y$ overconvergent along $Z$ (i.e. the category of coherent $j^{\dagger} \mathcal{O}_{\mathcal{X}_{K}}$-modules endowed with an overconvergent connection along $Z$ ) to that of coherent $(F-) \mathcal{D}_{\mathcal{X}}^{\dagger}\left({ }^{\dagger} Z\right)_{\mathbb{Q}}$-modules, coherent over $\mathcal{O}_{\mathcal{X}}\left({ }^{\dagger} Z\right)_{\mathbb{Q}}($ see $[$ Ber00, 4.6.7] and [Car06, 2.2.12]). Via this equivalence of categories, the constant coefficients correspond each other, i.e. $\operatorname{sp}_{*}\left(j^{\dagger} \mathcal{O}_{\mathcal{X}_{K}}\right)=\mathcal{O}_{\mathcal{X}}\left({ }^{\dagger} Z\right)_{\mathbb{Q}}$.

We denote by $\left({ }^{\dagger} Z\right): F$-Isoc $\left(\mathcal{X}^{b} / K\right) \rightarrow F$-Isoc ${ }^{\dagger \dagger}(\mathcal{X}, Z / K)$ the exact functor defined by posing $\left({ }^{\dagger} Z\right)(\mathcal{F}):=$ $\mathcal{D}_{\mathcal{X}}^{\dagger}\left({ }^{\dagger} Z\right)_{\mathbb{Q}} \otimes_{\mathcal{D}_{\mathcal{X}^{b}, \mathbb{Q}}^{\dagger}} \mathcal{F}$ for any $\mathcal{F} \in F$-Isoc $\left(\mathcal{X}^{b} / K\right)$ (indeed, by $\left.\left.[\mathrm{CT} 12,2.2 .1], \mathcal{D}_{\mathcal{X}}^{\dagger}\left({ }^{\dagger} Z\right)_{\mathbb{Q}}=\mathcal{D}_{\mathcal{X}^{b}}^{\dagger}{ }^{\dagger} Z\right)_{\mathbb{Q}}\right)$. We also put $\mathcal{F}\left({ }^{\dagger} Z\right):=\left({ }^{\dagger} Z\right)(\mathcal{F})$.

3.1.6. In the rest of the section, we fix $\left(\mathcal{F}, \phi_{\mathcal{F}}\right)$ an object of the abelian category $F$-Isoc $\left(\mathcal{X}^{b} / K\right)$, i.e., $\mathcal{F}$ is a coherent $\mathcal{D}_{\mathcal{X}^{b}, \mathbb{Q}^{-}}^{\dagger}$ module, projective of finite type over $\mathcal{O}_{\mathcal{X}, \mathbb{Q}}$ with nilpotent residues and $\phi_{\mathcal{F}}: F_{\mathcal{X}}^{*}(\mathcal{F}) \stackrel{\sim}{\rightarrow} \mathcal{F}$ is

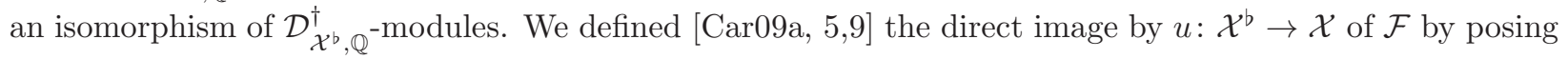

$$
u_{+}(\mathcal{F}):=\left(\mathcal{D}_{\mathcal{X}, \mathbb{Q}}^{\dagger} \otimes_{\mathcal{O}_{\mathcal{X}}} \mathcal{O}_{\mathcal{X}}(\mathcal{Z})\right) \otimes_{\mathcal{D}_{\mathcal{X}^{b}, \mathbb{Q}}^{\dagger}}^{\mathbb{L}} \mathcal{F}
$$

(see also the notation [Car09a, 5.3 and 5.8]). From Theorem [CT12, 2.2.9] (see the example [CT12, 2.2.10]), we get $u_{+}(\mathcal{F}) \stackrel{\sim}{\rightarrow} \mathcal{F}(\dagger Z)$. We put $\mathcal{E}=u_{+}(\mathcal{F})$ and we denote by $\phi_{\mathcal{E}}: F_{\mathcal{X}}^{*}(\mathcal{E}) \stackrel{\sim}{\rightarrow} \mathcal{E}$ the isomorphism induced by $\phi_{\mathcal{F}}$. In Kedlaya's terminology of [Ked07], $\mathcal{E}$ is associated (via the equivalence of categories sp ${ }_{*}$ ) to a unipotent overconvergent isocrystals with nilpotent residues. From the equivalence of categories of [Ked07, 6.4.5], we remark that the isomorphism $\phi_{\mathcal{F}}$ is determined by $\phi_{\mathcal{E}}$, i.e. is the unique one satisfying $\left({ }^{\dagger} Z\right)\left(\phi_{\mathcal{F}}\right)=\phi_{\mathcal{E}}$.

\subsection{Monodromy filtration of a convergent log-isocrystal with nilpotent residues}

We keep the notation of $\S 3.1$.

3.2.1. As in 3.1.5, we denote by $F$-Isoc $\left(\mathcal{Z}_{1}^{\#} / K\right)$ the abelian category of coherent $F-\mathcal{D}_{\mathcal{Z}_{1}^{\#}, \mathbb{Q}^{-m o d u l e s, ~ p r o j e c-~}}^{\dagger}$ tive of finite type over $\mathcal{O}_{\mathcal{Z}_{1}, \mathbb{Q}}$ and with nilpotent residues. If $V$ is an object of $F$-Isoc $\left(\mathcal{Z}_{1}^{\#} / K\right)$, the underlying $\mathcal{D}_{\mathcal{Z}_{1}^{\#}, \mathbb{Q}^{-}}^{\dagger}$-module is still denoted by $V$ and the Frobenius structure is denoted by $\phi_{V}$. For any integer $n$, for any $V \in F-\operatorname{Isoc}\left(\mathcal{Z}_{1}^{\#} / K\right)$, we put $V(n):=\left(V, q^{-n} \phi_{V}\right)$.

a) Let $V, V^{\prime} \in F$-Isoc $\left(\mathcal{Z}_{1}^{\#} / K\right)$. The object $\mathcal{H o m}_{\mathcal{O}_{\mathcal{Z}_{1}, \mathbb{Q}}}\left(V, V^{\prime}\right)$ of $\operatorname{Isoc}\left(\mathcal{Z}_{1}^{\#} / K\right)$ is canonically endowed with a Frobenius structure via the isomorphisms

$$
F_{\mathcal{Z}_{1}}^{*} \mathcal{H o m}_{\mathcal{O}_{\mathcal{Z}_{1}, \mathbb{Q}}}\left(V, V^{\prime}\right) \stackrel{\sim}{\rightarrow} \mathcal{H o m}_{\mathcal{O}_{z_{1}, \mathbb{Q}}}\left(F_{\mathcal{Z}_{1}}^{*} V, F_{\mathcal{Z}_{1}}^{*} V^{\prime}\right) \stackrel{\sim}{\rightarrow} \mathcal{H o m}_{\mathcal{O}_{z_{1}, \mathbb{Q}}}\left(V, V^{\prime}\right),
$$

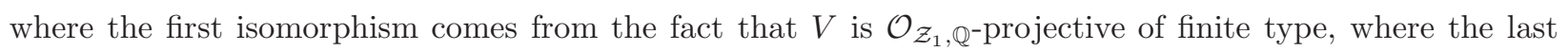
isomorphism is induced by $\phi_{V}^{-1}: V \stackrel{\sim}{\rightarrow} F_{\mathcal{Z}_{1}}^{*}(V)$ and $\phi_{V^{\prime}}: F_{\mathcal{Z}_{1}}^{*}\left(V^{\prime}\right) \stackrel{\sim}{\rightarrow} V^{\prime}$. Hence, $\mathcal{H o m}_{\mathcal{O}_{z_{1}, \mathbb{Q}}}\left(V, V^{\prime}\right)$ is also canonically an object of $F$-Isoc $\left(\mathcal{Z}_{1}^{\#} / K\right)$. In particular, $V^{\vee}:=\mathcal{H o m}_{\mathcal{O}_{\mathcal{Z}_{1}, \mathbb{Q}}}\left(V, \mathcal{O}_{\mathcal{Z}_{1}, \mathbb{Q}}\right) \in F$-Isoc $\left(\mathcal{Z}_{1}^{\#} / K\right)$. For any morphism $N: V \rightarrow V^{\prime}$ of $F$-Isoc $\left(\mathcal{Z}_{1}^{\#} / K\right)$, we denote by $N^{\vee}: V^{\prime \vee} \rightarrow V^{\vee}$ the morphism of $F$-Isoc $\left(\mathcal{Z}_{1}^{\#} / K\right)$ defined by $\phi \mapsto \phi \circ N$. If $\iota: W \hookrightarrow V$ is a monomorphism of $F$-Isoc $\left(\mathcal{Z}_{1}^{\#} / K\right)$, then we denote by $W^{\perp}$ the kernel of $\iota^{\vee}$.

b) In the same way, we denote by $V \otimes_{\mathcal{O}_{\mathcal{Z}_{1}, \mathbb{Q}}} V^{\prime} \in F-\operatorname{Isoc}\left(\mathcal{Z}_{1}^{\#} / K\right)$ the object $V \otimes_{\mathcal{O}_{\mathcal{Z}_{1}, \mathbb{Q}}} V^{\prime} \in \operatorname{Isoc}\left(\mathcal{Z}_{1}^{\#} / K\right)$ whose Frobenius structure is canonically induced by $\phi_{V}$ and $\phi_{V^{\prime}}$. 
c) For any integers $n, n^{\prime}$, we have $\mathcal{H o m}_{\mathcal{O}_{\mathcal{z}_{1}, \mathbb{Q}}}\left(V(n), V^{\prime}\left(n^{\prime}\right)\right)=\mathcal{H o m}_{\mathcal{O}_{z_{1}, \mathbb{Q}}}\left(V, V^{\prime}\right)\left(n^{\prime}-n\right),(V(n))^{\vee}=$ $V^{\vee}(-n), V(n) \otimes_{\mathcal{O}_{\mathcal{z}_{1}, \mathbb{Q}}} V^{\prime}\left(n^{\prime}\right)=\left(V \otimes_{\mathcal{O}_{\mathcal{z}_{1}, \mathbb{Q}}} V^{\prime}\right)\left(n+n^{\prime}\right)$.

Definition 3.2.2. We define the category $\mathfrak{C}$ as follows. An object $(V, N)$ of $\mathfrak{C}$ is the data of an object $V$ of $F$-Isoc $\left(\mathcal{Z}_{1}^{\#} / K\right)$ and a morphism $N: V(1) \rightarrow V$ of $F$-Isoc $\left(\mathcal{Z}_{1}^{\#} / K\right)$ which is a nilpotent morphism in

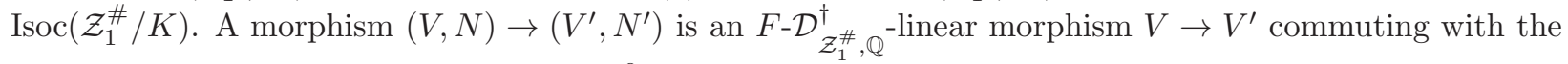
nilpotent endomorphisms. We denote by $\mathfrak{C}^{0}$ the full subcategory of $\mathfrak{C}$ of objects $(V, N)$ such that $N=0$. We check easily that both categories $\mathfrak{C}$ and $\mathfrak{C}^{0}$ are abelian.

Definition 3.2.3. Let $(V, N),\left(V^{\prime}, N^{\prime}\right) \in \mathfrak{C}$. We define the following operations in $\mathfrak{C}$ :

1. The internal Hom of $(V, N)$ and $\left(V^{\prime}, N^{\prime}\right)$ in $\mathfrak{C}$ is $\left.\mathcal{H o m}_{\mathcal{O}_{\mathcal{Z}_{1}, \mathbb{Q}}}\left((V, N),\left(V^{\prime}, N^{\prime}\right)\right):=\left(\mathcal{H}_{0} m_{\mathcal{O}_{\mathcal{Z}_{1}, \mathbb{Q}}}\left(V, V^{\prime}\right), N^{\prime \prime}\right)\right)$, where for any $\phi \in \mathcal{H o m}_{\mathcal{O}_{\mathcal{Z}_{1}, \mathbb{Q}}}\left(V, V^{\prime}\right)(1)$, we put $N^{\prime \prime}(\phi):=N^{\prime} \circ \phi-\phi \circ N(-1)$.

2. In particular, the dual of $(V, N)$ in $\mathfrak{C}$ is $(V, N)^{\vee}:=\left(V^{\vee},-N(-1)^{\vee}\right)$.

3. The tensor product of $(V, N)$ and $\left(V^{\prime}, N^{\prime}\right)$ in $\mathfrak{C}$ is $(V, N) \otimes_{\mathcal{O}_{z_{1}, \mathbb{Q}}}\left(V^{\prime}, N^{\prime}\right):=\left(V \otimes_{\mathcal{O}_{\mathcal{z}_{1}, \mathbb{Q}}} V^{\prime}, N \otimes \mathrm{id}+\mathrm{id} \otimes N^{\prime}\right)$.

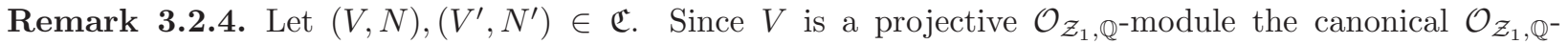
linear morphism $V^{\vee} \otimes_{\mathcal{O}_{\mathcal{Z}_{1}, \mathbb{Q}}} V^{\prime} \rightarrow \mathcal{H}^{\prime} m_{\mathcal{O}_{z_{1}, \mathbb{Q}}}\left(V, V^{\prime}\right)$ is an isomorphism. We can show that this isomorphism commutes with the canonical nilpotent endomorphisms, and induces an isomorphism in $\mathfrak{C}$.

Lemma 3.2.5. Let $(V, N) \in \mathfrak{C}$. Then, the evaluation homomorphism induces the canonical isomorphism: $\mathrm{ev}:(V, N) \stackrel{\sim}{\rightarrow}\left((V, N)^{\vee}\right)^{\vee}$. Moreover, we get the canonical isomorphisms of $\mathfrak{C}^{0}$ :

$$
(\operatorname{ker} N(-1))^{\vee} \stackrel{\sim}{\rightarrow} \operatorname{coker}\left(N(-1)^{\vee}\right), \quad(\operatorname{coker} N)^{\vee} \stackrel{\sim}{\rightarrow} \operatorname{ker}\left(N^{\vee}\right) .
$$

Proof. The proof is straightforward.

Definition 3.2.6 (Monodromy filtration). Let $(V, N) \in \mathfrak{C}$. Copying the proof of [Del80, 1.6.1], we check that there exists a unique finite increasing filtration $M$ on $V$ such that $N M_{i} \subset M_{i-2}(-1)$, and $N^{k}$ induces an isomorphism $\operatorname{gr}_{k}^{M}(V) \stackrel{\sim}{\rightarrow} \operatorname{gr}_{-k}^{M}(V)(-k)$. We call this filtration the monodromy filtration and it will usually be denoted by $M$.

Remark 3.2.7. Let $\omega: F$-Isoc $\left(\mathcal{Z}_{1}^{\#} / K\right) \rightarrow \operatorname{Isoc}^{0}\left(\mathcal{Z}_{1}^{\#} / K\right)$ be the forgetful functor. Let $\mathfrak{E}$ be the category defined in the same way as $\mathfrak{C}$ but without Frobenius structure (i.e., we replace the abelian category $F$-Isoc $\left(\mathcal{Z}_{1}^{\#} / K\right)$ by $\left.\operatorname{Isoc}^{0}\left(\mathcal{Z}_{1}^{\#} / K\right)\right)$ and let $\omega: \mathfrak{C} \rightarrow \mathfrak{E}$ be the forgetful functor defined by $\omega(V, N)=$ $(\omega(V), \omega(N))$. For any $(V, N) \in \mathfrak{C}$, from [Del80, 1.6.1], we have a monodromy filtration on $\omega(V, N)$ denoted by $M^{\prime}$. Then by the uniqueness property of monodromy filtration we get that $\omega\left(M_{i}\right) \cong M_{i}^{\prime}$.

3.2.8. Let $(V, N),\left(V^{\prime}, N^{\prime}\right),\left(V^{\prime \prime}, N^{\prime \prime}\right) \in \mathfrak{C}$. As in $[\operatorname{Del} 80,1.6 .9]$, with the notation 3.2 .1 , we put

$$
M_{i}\left((V, N)^{\vee}\right):=M_{-i-1}(V, N)^{\perp}, \quad M_{i}\left(\left(V^{\prime}, N^{\prime}\right) \otimes_{\mathcal{O}_{\mathcal{Z}_{1}, \mathbb{Q}}}\left(V^{\prime \prime}, N^{\prime \prime}\right)\right):=\sum_{i^{\prime}+i^{\prime \prime}=i} M_{i^{\prime}}\left(V^{\prime}, N^{\prime}\right) \otimes_{\mathcal{O}_{\mathcal{Z}_{1}, \mathbb{Q}}} M_{i^{\prime \prime}}\left(V^{\prime \prime}, N^{\prime \prime}\right) .
$$

From the formulas

$$
\begin{gathered}
\operatorname{gr}_{i}^{M}\left((V, N)^{\vee}\right)=M_{-i-1}(V, N)^{\perp} / M_{-i}(V, N)^{\perp} \stackrel{\sim}{\longrightarrow}\left(M_{-i}(V, N) / M_{-i-1}(V, N)\right)^{\vee}=\operatorname{gr}_{-i}^{M}(V, N), \\
\operatorname{gr}_{i}^{M}\left(\left(V^{\prime}, N^{\prime}\right) \otimes_{\mathcal{O}_{\mathcal{z}_{1}, \mathbb{Q}}}\left(V^{\prime \prime}, N^{\prime \prime}\right)\right)=\sum_{i^{\prime}+i^{\prime \prime}=i} \operatorname{gr}_{i^{\prime}}^{M}\left(V^{\prime}, N^{\prime}\right) \otimes_{\mathcal{O}_{\mathcal{z}_{1}, \mathbb{Q}}} \operatorname{gr}_{i^{\prime \prime}}^{M}\left(V^{\prime \prime}, N^{\prime \prime}\right),
\end{gathered}
$$

we check that these filtrations on $(V, N)^{\vee}$ and $\left(V^{\prime}, N^{\prime}\right) \otimes_{\mathcal{O}_{z_{1}, \mathbb{Q}}}\left(V^{\prime \prime}, N^{\prime \prime}\right)$ are the monodromy filtrations (which justifies the notation). 
3.2.9 (Remarks on the induced filtration by inclusion and quotient on respectively $\operatorname{ker} N$ and $\operatorname{coker} N$ ). Let $(V, N) \in \mathfrak{C}$. By construction of the monodromy filtration (see the proof of [Del80, 1.6.1] for the explicit construction), we check by induction on the smallest integer $d \in \mathbb{N}$ such that $N^{d+1}=0$ that ker $N(-1) \subset M_{0}(V, N)$. In particular, denoting (by abuse of notation) by $M_{i}($ ker $N(-1))$ the induced filtration on $\operatorname{ker} N(-1)$ (from the monodromy filtration of $(V, N)$ by inclusion), we $\operatorname{get}^{\operatorname{gr}_{i}^{M}}(\operatorname{ker} N(-1))=0$ for $i>0$.

Moreover, since we have in $(V, N)^{\vee}$ the inclusion $M_{-1}\left((V, N)^{\vee}\right)=M_{0}(V, N)^{\perp} \subset \operatorname{ker} N(-1)^{\perp}$, the image of $M_{-1}\left((V, N)^{\vee}\right)$ throught $V^{\vee} \rightarrow(\operatorname{ker} N(-1))^{\vee} \underset{(3.2 .5 .1)}{\stackrel{\sim}{\longrightarrow}} \operatorname{coker}\left(N(-1)^{\vee}\right)=\operatorname{coker}\left(N_{V^{\vee}}\right)$, where $N_{V^{\vee}}=-N(-1)^{\vee}$ is the the nilpotent morphism of $(V, N)^{\vee}$, is null. Denoting (by abuse of notation) by $M_{i}$ coker $\left(N_{V^{\vee}}\right)$ the induced filtration on $\operatorname{coker}\left(N_{V^{\vee}}\right)$ (from the monodromy filtration of $V^{\vee}$ by quotient), we get that $M_{-1} \operatorname{coker}\left(N_{V^{\vee}}\right)=0$. Using the biduality isomorphism of lemma 3.2.5, denoting by $M_{i} \operatorname{coker}(N)$ the induced filtration on $\operatorname{coker}(N)$ (from the monodromy filtration of $V$ by quotient), we get that $M_{-1} \operatorname{coker}(N)=0$. In particular, we $\operatorname{get} \operatorname{gr}_{i}^{M} \operatorname{coker} N=0$ if $i<0$.

3.2.10 (The functor $i_{1}^{*}$ ). Let $i_{1}^{b}: \mathcal{Z}_{1}^{\#} \hookrightarrow \mathcal{X}^{b}$ be the closed immersion. By functoriality, $\mathcal{D}_{\mathcal{Z}_{1}^{\#} \rightarrow \mathcal{X}^{b}}:=i_{1}^{*} \mathcal{D}_{\mathcal{X}^{b}}$ is a $\left(\mathcal{D}_{\mathcal{Z}_{1}^{\#}}, \mathcal{D}_{\mathcal{X}^{b}}\right)$-bimodule, where $i_{1}^{*}:=\mathcal{O}_{\mathcal{Z}_{1}} \otimes_{i_{1}^{-1} \mathcal{O}_{\mathcal{X}}}-$. We get the functor $i_{1}^{b !}: D^{-}\left(\mathcal{D}_{\mathcal{X}^{b}, \mathbb{Q}}\right) \rightarrow D^{-}\left(\mathcal{D}_{\mathcal{Z}_{1, \mathbb{Q}}^{\#}}\right)$, by posing $i_{1}^{b !}(\mathcal{G}):=\mathcal{D}_{\mathcal{Z}_{1}^{\#} \rightarrow \mathcal{X}^{b}, \mathbb{Q}} \otimes_{\mathcal{D}_{\mathcal{X}^{b}, \mathbb{Q}}}^{\mathbb{L}} \mathcal{G}[-1]$, for any $\mathcal{G} \in D^{-}\left(\mathcal{D}_{\mathcal{X}^{b}, \mathbb{Q}}\right)$. Similarly (see 3.1.5), we have the functor $F_{\mathcal{Z}_{1}^{\#}}^{!}: D^{-}\left(\mathcal{D}_{\mathcal{Z}_{1, \mathbb{Q}}^{\#}}\right) \rightarrow D^{-}\left(\mathcal{D}_{\mathcal{Z}_{1, \mathbb{Q}}^{\#}}\right)$ (resp. $F_{\mathcal{X}^{b}}^{!}: D^{-}\left(\mathcal{D}_{\mathcal{X}^{b}, \mathbb{Q}}\right) \rightarrow D^{-}\left(\mathcal{D}_{\mathcal{X}^{b}, \mathbb{Q}}\right)$. By transitivity (in this context of $\mathcal{D}$-modules and not $\mathcal{D}^{\dagger}$-modules, we do not have to deal with the highly technical notion of Berthelot's quasicoherence as in [Ber02]), we get the isomorphism $i_{1}^{b !} \circ F_{\mathcal{X}^{b}}^{!} \stackrel{\sim}{\rightarrow} F_{\mathcal{Z}_{1}^{\#}}^{!} \circ i_{1}^{b !}$ of functors $D^{-}\left(\mathcal{D}_{\mathcal{X}^{b}, \mathbb{Q}}\right) \rightarrow D^{-}\left(\mathcal{D}_{\mathcal{Z}_{1, \mathbb{Q}}^{\#}}\right)$.

By flatness and preservation of the notion of Kedlaya's convergence (as explained in 3.1.4), the functor $i_{1}^{b !}[1]$ factors through $i_{1}^{b !}[1]: \operatorname{Isoc}\left(\mathcal{X}^{b} / K\right) \rightarrow \operatorname{Isoc}\left(\mathcal{Z}_{1}^{\#} / K\right)$. We denote by $i_{1}^{*}$ this functor. Similarly (see 3.1.5), the factorizations $F_{\mathcal{Z}_{1}^{\#}}^{!}: \operatorname{Isoc}\left(\mathcal{Z}_{1}^{\#} / K\right) \rightarrow \operatorname{Isoc}\left(\mathcal{Z}_{1}^{\#} / K\right)$ and $F_{\mathcal{X}^{b}}^{!}: \operatorname{Isoc}\left(\mathcal{X}^{b} / K\right) \rightarrow \operatorname{Isoc}\left(\mathcal{X}^{b} / K\right)$, are denoted by $F_{\mathcal{Z}_{1}}^{*}$ and $F_{\mathcal{X}}^{*}$. Hence, the functor $i_{1}^{*}$ commutes canonically with Frobenius, i.e. we get the factorisation:

$$
i_{1}^{*}: F-\operatorname{Isoc}\left(\mathcal{X}^{b} / K\right) \rightarrow F-\operatorname{Isoc}\left(\mathcal{Z}_{1}^{\#} / K\right)
$$

We remark that $i_{1}^{*}$ is equal to the cokernel of the multiplication by $t_{1}$, i.e. $i_{1}^{*}(\mathcal{G})=\mathcal{G} / t_{1} \mathcal{G}$ for any $\mathcal{G} \in F-\operatorname{Isoc}\left(\mathcal{X}^{b} / K\right)$.

3.2.11 (Monodromy filtrations coming from $\mathcal{F})$. We put $\mathcal{H}:=i_{1}^{*}(\mathcal{F}) \in F$-Isoc $\left(\mathcal{Z}_{1}^{\#} / K\right)$ (recall that $\mathcal{F}$ is defined in 3.1.6 and the functor $i_{1}^{*}$ is defined in (3.2.10.1)). The action of $t_{1} \partial_{1}$ on $\mathcal{F}$ induces the residue

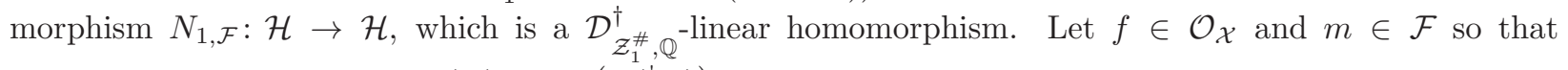
$f \otimes m \in \mathcal{O}_{\mathcal{X}} \otimes_{F_{\mathcal{X}}, \mathcal{O}_{\mathcal{X}}} \mathcal{F}=F_{\mathcal{X}}^{*}(\mathcal{F}) \subset F_{\mathcal{X}}^{*}\left(\mathcal{F}\left({ }^{\dagger} Z\right)\right)$, where $F_{\mathcal{X}}: \mathcal{X} \rightarrow \mathcal{X}$ is the canonical Frobenius defined in 3.1.3. For any $1 \leq i \leq d$, we have

$$
t_{i} \partial_{i}(f \otimes m)=t_{i} \partial_{i}(f) \otimes m+f \otimes q t_{i} \partial_{i}(m)
$$

in $F_{\mathcal{X}}^{*}(\mathcal{F})$. Now, we define the following Frobenius structure on $\mathcal{H}$ via the composition

$$
\phi_{\mathcal{H}}: F_{\mathcal{Z}_{1}}^{*} \mathcal{H} \stackrel{\sim}{\longrightarrow} i_{1}^{*} F_{\mathcal{X}}^{*}(\mathcal{F}) \underset{i_{1}^{*} \phi_{\mathcal{F}}}{\stackrel{\sim}{\longrightarrow}} i_{1}^{*}(\mathcal{F})=\mathcal{H}
$$

Using the computation (3.2.11.1), we check that the left square of the following diagram is commutative:

$$
\begin{aligned}
& F_{\mathcal{Z}_{1}}^{*} \mathcal{H} \stackrel{\sim}{\longrightarrow} i_{1}^{*} F_{\mathcal{X}}^{*}(\mathcal{F}) \stackrel{i_{1}^{*}\left(\phi_{\mathcal{F}}\right)}{\longrightarrow} i_{1}^{*}(\mathcal{F}) \\
& q F_{\mathcal{Z}_{1}}^{*}\left(N_{1, \mathcal{F}} \downarrow\right. N_{1, F_{\mathcal{X}}^{*} \mathcal{F} \downarrow} \\
& F_{\mathcal{Z}_{1}}^{*} \mathcal{H} \longrightarrow i_{1}^{*} F_{\mathcal{X}}^{*}(\mathcal{F}) \stackrel{i_{1}^{*}\left(\phi_{\mathcal{F}}\right)}{\longrightarrow} i_{1}^{*}(\mathcal{F}) .
\end{aligned}
$$

Since the right square is commutative by functoriality, the diagram (3.2.11.3) is commutative. The commutativity of the diagram (3.2.11.3) means that the homomorphism $N_{1, \mathcal{F}}$ induces the homomorphism 
$N_{1, \mathcal{F}}: \mathcal{H}(1) \rightarrow \mathcal{H}$ in $F$-Isoc $\left(\mathcal{Z}_{1}^{\#} / K\right)$. Since $\mathcal{F}$ has nilpotent residues, then by definition $N_{1, \mathcal{F}}$ is nilpotent. In other words, we have checked $\left(\mathcal{H}, N_{1, \mathcal{F}}\right) \in \mathfrak{C}$. As in 3.2.6, we denote by $\left(M_{i}\left(\mathcal{H}, N_{1, \mathcal{F}}\right)\right)_{i \in \mathbb{Z}}$ the monodromy filtration on $\left(\mathcal{H}, N_{1, \mathcal{F}}\right)$.

\subsection{Comparison with Crew's Frobenius structure}

3.3.1 (Notation: curve case). Using the notation of $\S 3.1$, suppose in this paragraph that $X$ is of dimension 1 and that $Z$ is $k$-rational. In this context, we simply denote $t_{1}$ by $t$ and $\partial_{1}$ by $\partial$. In that case, from [Ked07, 4.3.3], we get $\left.A_{K}^{1}[0,1) \stackrel{\sim}{\rightarrow}\right] Z\left[\mathcal{X}\right.$, where $A_{K}^{1}[0,1)$ is the analytic subspace of the analytic line $\mathbb{A}_{K}^{1, \text { an }}$ of the elements $t \in K$ such that $|t|<1$ (see the notation $[\operatorname{Ked} 07,3.1 .2]$ ). We put $\mathcal{O}_{K}^{\text {an }}:=\Gamma\left(A_{K}^{1}[0,1), \mathcal{O}_{A_{K}^{1}[0,1)}\right.$ ). Hence, $\mathcal{O}_{K}^{\text {an }}$ is the subring of $K[[t]]$ of power series in $t$ convergent for $|t|<1$. We denote by $\mathcal{R}_{K}$ the Robba ring relative to $K$. We recall this Robba ring $\mathcal{R}_{K}$ is the ring of formal Laurent series $f=\sum_{n \in \mathbb{Z}} a_{n} t^{n}$ in $t$ with coefficients in $K$, convergent in some thin annulus $r<|t|<1$ for some real number $0<r<1$ (depending on $f)$. We remark that $\mathcal{R}_{K}$ is equal to $\Gamma(] Z\left[\mathcal{X}, j^{\dagger} \mathcal{O}_{\mathcal{X}_{K}}\right)$.

We put $F:=\Gamma(\mathcal{X}, \mathcal{F}), F_{] Z[}:=\Gamma(] Z\left[\mathcal{X}, \mathrm{sp}^{*}(\mathcal{E})\right)=\mathcal{O}_{K}^{\text {and }} \otimes_{A_{K}} F, E:=\Gamma(\mathcal{X}, \mathcal{E})$ and $E_{] Z}:=\Gamma(] Z\left[\mathcal{X}, \mathrm{sp}^{*}(\mathcal{E})\right)=$ $\mathcal{R}_{K} \otimes_{A\left[\frac{1}{t}\right]_{K}^{\dagger}} E$. We have the canonical Frobenius endomorphisms $F^{*}: \mathcal{O}_{K}^{\text {an }} \rightarrow \mathcal{O}_{K}^{\text {an }}$ (resp. $F^{*}: \mathcal{R}_{K} \rightarrow$ $\mathcal{R}_{K}$ ) given by $\sum_{n \in \mathbb{N}} a_{n} t^{n} \mapsto \sum_{n \in \mathbb{N}} a_{n} t^{q n}$ (resp. $\left.\quad \sum_{n \in \mathbb{Z}} a_{n} t^{n} \mapsto \sum_{n \in \mathbb{Z}} a_{n} t^{q n}\right)$. We put $F^{*}\left(F_{] Z[}\right):=$ $\mathcal{O}_{K}^{\text {an }} \otimes_{F, \mathcal{O}_{K}^{\text {an }}} F_{] Z[}, F^{*}\left(E_{] Z[}\right):=\mathcal{R}_{K} \otimes_{F, \mathcal{R}_{K}} E_{Z[Z}$. We set $F_{] Z[}^{\nabla^{\infty}}:=\bigcup_{n>0} \operatorname{ker}\left((t \partial)^{n}: F_{] Z[} \rightarrow F_{] Z[}\right)$ and $E_{] Z[}^{\nabla^{\infty}}:=$ $\cup_{n>0} \operatorname{ker}\left((t \partial)^{n}: E_{] Z[} \rightarrow E_{] Z[}\right)$. We remark that the $K$-linear endomorphism induced by $t \partial$ on $E_{] Z[}^{\nabla^{\infty}}$ (resp. $\left.F_{] Z[}^{\nabla^{\infty}}\right)$ is nilpotent. The monomorphism of $K$-vector spaces $E_{] Z[}^{\nabla^{\infty}} \subset E_{] Z[}$ induces by extension the canonical morphism can : $\mathcal{R}_{K} \otimes_{K} E_{] Z[}^{\nabla^{\infty}} \rightarrow E_{] Z[}$. Similarly we get the canonical morphism can: $\mathcal{O}_{K}^{\text {an }} \otimes_{K} F_{] Z[}^{\nabla^{\infty}} \rightarrow F_{] Z[}$. We denote by $i_{1}^{*}$ the cokernel of the multiplication by $t$. To simplify notation, we denote by $\phi$ the Frobenius structure on $F, F_{Z}\left[, E_{] Z}\right.$, which are induced by extension from that on $F$.

3.3.2 (Unipotence and consequences). We keep the hypotheses and notation of the paragraph 3.3.1. Following $[\operatorname{Ked07}, 3.6 .9], E_{] Z[}$ is unipotent. This implies that the canonical morphism can: $\mathcal{R}_{K} \otimes_{K} E_{] Z[}^{\nabla^{\infty}} \rightarrow E_{] Z[}$ (e.g. see $\left[\right.$ Mat02, 4.1]). In particular, $E_{] Z[}^{\nabla^{\infty}}$ is a $K$-vector space of dimension equal to the rank of $E_{] Z[}$ as $\mathcal{R}_{K}$-module. In fact, from the nilpotent endomorphism induced by $t \partial$ on $E_{] Z[}^{\nabla^{\infty}}$, we get a canonical connection on $\mathcal{R}_{K} \otimes_{K} E_{] Z[}^{\nabla^{\infty}}$ and this canonical isomorphism can: $\mathcal{R}_{K} \otimes_{K} E_{] Z[}^{\nabla^{\infty}} \stackrel{\sim}{\rightarrow} E_{] Z[}$ is in fact an isomorphism of $\left(\mathcal{R}_{K}, \nabla\right)$-modules. Following [Ked07, 3.6.2] (this is more explicitely written in [Shi10, 2.12] and also more general), since $F_{] Z[}$ is log-convergent then $F_{] Z[}$ is unipotent. Hence, the canonical morphism can: $\mathcal{O}_{K}^{\text {an }} \otimes_{K} F_{] Z[}^{\nabla^{\infty}} \rightarrow F_{] Z[}$ is an isomorphism. Since $A\left[\frac{1}{t}\right]_{K}^{\dagger} \otimes_{A_{K}} F \stackrel{\sim}{\rightarrow} E$, we check that the canonical morphism $\mathcal{R}_{K} \otimes_{\mathcal{O}_{K}^{\text {an }}} F_{] Z[} \rightarrow E_{] Z[}$ is an isomorphism. This yields that the dimension of the $K$-vector space $F_{] Z[}^{\nabla^{\infty}}$ is equal to that of $E_{] Z[}^{\nabla^{\infty}}$. Hence, the canonical inclusion $F_{] Z[}^{\nabla^{\infty}} \subset E_{] Z[}^{\nabla^{\infty}}$ is a $K$-isomorphism.

3.3.3 (Crew's Frobenius structure on $E_{] Z[}^{\nabla^{\infty}}$ ). We keep the hypotheses and notation of the paragraph 3.3.1. Since the canonical Frobenius endomorphism $F^{*}: \mathcal{R}_{K} \rightarrow \mathcal{R}_{K}$ (the local coordinate $t$ is fixed) is $K$-linear, we have $F^{*}\left(\mathcal{R}_{K} \otimes_{K} E_{] Z[}^{\nabla^{\infty}}\right) \stackrel{\sim}{\rightarrow} \mathcal{R}_{K} \otimes_{K} F^{*}\left(E_{] Z[}^{\nabla^{\infty}}\right)=\mathcal{R}_{K} \otimes_{K} E_{] Z[}^{\nabla^{\infty}}$. Similarly, we get $F^{*}\left(\mathcal{O}_{K}^{\text {an }} \otimes_{K} F_{] Z[}^{\nabla^{\infty}}\right) \stackrel{\sim}{\rightarrow}$ $\mathcal{O}_{K}^{\text {an }} \otimes_{K} F_{] Z[}^{\nabla^{\infty}}$. We define $\psi_{\eta}$ and $\psi$ so that the corresponding squares of the diagram

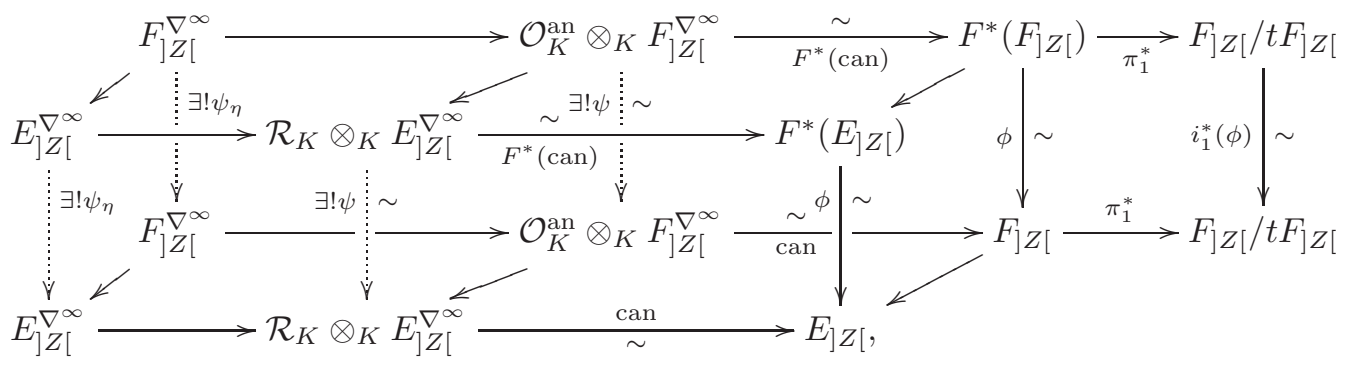


where $\pi_{1}^{*}$ is the canonical projection, are commutative (the existence and unicity of $\psi$ is obvious, for that of $\psi_{\eta}$ we use the fact that $\psi$ commute with connections). The isomorphism $\psi_{\eta}: E_{] Z[}^{\nabla^{\infty}} \stackrel{\sim}{\rightarrow} E_{] Z[}^{\nabla^{\infty}}$ that we get is Crew's Frobenius action defined in [Cre98, §10]. The isomorphism $\psi_{\eta}: F_{] Z[}^{\nabla^{\infty}} \stackrel{\sim}{\rightarrow} F_{] Z[}^{\nabla^{\infty}}\left(\right.$ resp. $i_{1}^{*}(\phi): F_{] Z[} / t F_{] Z[} \stackrel{\sim}{\rightarrow}$ $F_{] Z[} / t F_{] Z[}$ ) will be by definition the Frobenius structure on $F_{] Z[}^{\nabla^{\infty}}\left(\right.$ resp. $F_{] Z[}^{\nabla^{\infty}}$ ) induced canonically from that of $\mathcal{F}$.

Lemma 3.3.4 (Comparison with Crew's Frobenius structure). We keep the hypotheses of 3.3.1. We have a canonical $K$-isomorphism of the form

$$
E_{] Z[}^{\nabla^{\infty}} \stackrel{\sim}{\rightarrow} F / t F
$$

which commutes with Frobenius (Crew's Frobenius structure of 3.3 .3 for the left term and that defined in (3.2.11.2) for the right term) and with the (nilpotent) monodromy operation induced by t2. In particular, the monodromy filtrations correspond.

Proof. From the commutativity of the left square of the diagram (3.3.3.1), the isomorphism $F_{] Z[}^{\nabla^{\infty}} \stackrel{\sim}{\rightarrow} E_{] Z[}^{\nabla^{\infty}}$ commutes with Frobenius structures (denoted there by $\psi_{\eta}$ ) and with the action of $t \partial$. We compute that the composition of the horizontal arrows $F_{] Z[}^{\nabla^{\infty}} \rightarrow F_{] Z[} / t F_{] Z[}$ of the diagram (3.3.3.1) is a $K$-isomorphism with commutes with the actions induced by $t \partial$, and from the commutativity of the diagram (3.3.3.1), this isomorphism commutes with Frobenius. On the other hand, by applying the functor $i_{1}^{*}$ to $F \rightarrow F_{] Z}$, we get an isomorphism commuting with Frobenius and with the actions induced by $t \partial$. Hence we get by composition the canonical $K$-isomorphism $E_{] Z[}^{\nabla^{\infty}} \stackrel{\sim}{\rightarrow} F / t F$ satisfying the required properties.

The next Theorem is an easy consequence of Crew's Theorem [Cre98, Theorem 10.8] and can be seen as a translation in the context of arithmetic $\mathcal{D}$-modules.

Theorem 3.3.5 (Crew). With the notation of $\S 3.1$, assume that $\mathcal{E}$ is $\iota$-pure of weight $w$ and that $\mathcal{E}$ comes from an overconvergent $F$-isocrystals on $Y$. Then $\left({ }^{\dagger} D_{1}\right)\left(\operatorname{gr}_{i}^{M}\left(\mathcal{H}, N_{1, \mathcal{F}}\right)\right) \in F-\operatorname{Isoc}^{\dagger \dagger}\left(\mathcal{Z}_{1}, D_{1} / K\right)$ is $\iota$-pure of weight $w+1+i$, where $\left({ }^{\dagger} D_{1}\right):=\mathcal{D}_{\mathcal{Z}_{1}}^{\dagger}\left({ }^{\dagger} D_{1}\right)_{\mathbb{Q}} \otimes_{\mathcal{D}_{\mathcal{Z}_{1}^{\dagger}, \mathbb{Q}}^{\dagger}}-: F$-Isoc $\left(\mathcal{Z}_{1}^{\#} / K\right) \rightarrow F$-Isoc ${ }^{\dagger \dagger}\left(\mathcal{Z}_{1}, D_{1} / K\right)$ is the functor defined in 3.1.5 and where $M$ is the monodromy filtration as defined in 3.2.11.

Proof. Let $x$ be a closed point of $\mathcal{Z}_{1} \backslash \mathfrak{D}_{1}, k(x)$ its residue field and $\mathcal{V}_{x}$ a finite étale $\mathcal{V}$-algebra which is a lifting of $k(x)$. We denote by $i_{x}: \operatorname{Spf} \mathcal{V}_{x} \hookrightarrow \mathcal{Z}_{1}$ a lifting of the induced closed immersion by $x$. We denote Spf $\mathcal{V}_{x}$ by $\{x\}$. We have to check that $i_{x}^{!} \operatorname{gr}_{i}^{M}\left(\mathcal{H}, N_{1, \mathcal{F}}\right)\left({ }^{\dagger} D_{1}\right)$ is $\iota$-pure of weight $w+1+i$. Up to a change of basis of $\mathcal{X}$ by the extension $\mathcal{V} \rightarrow \mathcal{V}_{x}$, we can suppose $k=k(x)$ by Lemma 2.2.10. Moreover, we can suppose that $\mathfrak{D}_{1}$ is empty (i.e $\mathcal{Z}=\mathcal{Z}_{1}$ ) and $\mathcal{Z} \cap V\left(t_{2}-t_{2}(x), \ldots, t_{d}-t_{d}(x)\right)=\{x\}$. Let $\mathcal{X}_{1}:=\operatorname{Spf} A_{1}:=$ $V\left(t_{2}-t_{2}(x), \ldots, t_{d}-t_{d}(x)\right), \mathcal{Y}_{1}:=\mathcal{X}_{1} \backslash \mathcal{Z}, \alpha: \mathcal{X}_{1} \hookrightarrow \mathcal{X}, \mathcal{X}_{1}^{\#}:=\left(\mathcal{X}_{1},\{x\}\right), \alpha^{\#}: \mathcal{X}_{1}^{\#} \hookrightarrow \mathcal{X}^{\#}, \beta:\{x\} \hookrightarrow \mathcal{Z}$, $\mathcal{F}_{1}:=\alpha^{\# *}(\mathcal{F}) \in F$-Isoc $\left(\mathcal{X}_{1}^{\#} / K\right), \mathcal{H}_{1}:=\mathcal{F}_{1} / t_{1} \mathcal{F}_{1}, \mathcal{E}_{1}:=\mathcal{F}_{1}\left({ }^{\dagger}\{x\}\right)$. Since $t_{1} \partial_{1}$ commutes with $\alpha^{\#}$, we get $\beta^{*} \operatorname{gr}_{i}^{M}\left(\mathcal{H}, N_{1, \mathcal{F}}\right) \stackrel{\sim}{\rightarrow} \operatorname{gr}_{i}^{M}\left(\mathcal{H}_{1}, N_{1, \mathcal{F}_{1}}\right)$, where the filtrations are the monodromy filtrations. Hence, we reduce to the curve case, i.e. $\mathcal{X}_{1}=\mathcal{X}$. Then, by using the comparison between our setting and Crew's one of the paragraph 3.3.4 and the remarks of 2.1.4, we apply [Cre98, Theorem 10.8].

Corollary 3.3.6. With the notation of 3.3.5, the object $\left({ }^{\dagger} D_{1}\right)\left(\operatorname{ker}\left(N_{1, \mathcal{F}}\right)(-1)\right)\left(\right.$ resp. $\left.\left({ }^{\dagger} D_{1}\right)\left(\operatorname{coker}\left(N_{1, \mathcal{F}}\right)\right)\right)$

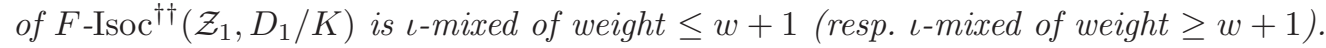

Proof. Since $\left({ }^{\dagger} D_{1}\right)$ is exact, the monodromy filtration of $\left(\mathcal{H}, N_{1, \mathcal{F}}\right) \in \mathfrak{C}$ induces a filtration on $\left({ }^{\dagger} D_{1}\right)(\mathcal{H})$ and then (as subobject and respectively quotient) on $\left(\operatorname{ker} N_{1, \mathcal{F}}(-1)\right)\left({ }^{\dagger} D_{1}\right)$ and (coker $\left.N_{1, \mathcal{F}}\right)\left({ }^{\dagger} D_{1}\right)$. Denoting these filtrations by $M$, with the Remark 3.2.9, we get $\operatorname{gr}_{i}^{M}\left(\left(\operatorname{ker} N_{1, \mathcal{F}}(-1)\right)\left({ }^{\dagger} D_{1}\right)\right)=0$ for $i>0$ and $\operatorname{gr}_{i}^{M}\left(\operatorname{coker} N_{1, \mathcal{F}}\left({ }^{\dagger} D_{1}\right)\right)=0$ if $i<0$. We conclude the proof by using Theorem 3.3.5. 


\subsection{Relations between a convergent log-isocrystal and its associated overcon- vergent isocrystal}

In this subsection, we keep the notation of $\S 3.1$ and we denote by $j:=(\star$, id, id $):(Y, X, \mathcal{X}) \rightarrow(X, X, \mathcal{X})$ the canonical morphism of frames. The aim of this subsection is to check Theorem 3.4.19.

Notation 3.4.1 (Dual functors, holonomicity). Let $\mathcal{P}$ be a smooth formal scheme over $\mathcal{V}$ and $\mathfrak{E}$ be a strict normal crossing divisor of $\mathcal{P}$ relatively to $\mathcal{V}$. We denote by $\mathcal{P}^{\#}:=(\mathcal{P}, \mathfrak{E})$ the corresponding $\log$ smooth formal scheme over $\mathcal{V}$ whose $\log$ structure is induced by $\mathfrak{E}$. Let $T$ be a divisor of the special fiber of $\mathcal{P}$. We denote by $D_{\text {parf }}\left(\mathcal{D}_{\mathcal{P} \#}^{\dagger}\left({ }^{\dagger} T\right)_{\mathbb{Q}}\right)$ the derived category of perfect complexes of left $\mathcal{D}_{\mathcal{P} \#}^{\dagger}\left({ }^{\dagger} T\right)_{\mathbb{Q}}$-modules. When $T$ is empty, we have $D_{\text {parf }}\left(\mathcal{D}_{\mathcal{P} \#, \mathbb{Q}}^{\dagger}\right)=D_{\text {coh }}^{\mathrm{b}}\left(\mathcal{D}_{\mathcal{P} \#, \mathbb{Q}}^{\dagger}\right)$ (see [Car09a, 5.5]). When $T$ is not empty, we do not know so far if we have such equality.

a) Following [Car09a, 5.17], we have the dual functor

$$
\mathbb{D}_{\mathcal{P} \#, T}: D_{\text {parf }}\left(\mathcal{D}_{\mathcal{P} \#}^{\dagger}\left({ }^{\dagger} T\right)_{\mathbb{Q}}\right) \rightarrow D_{\text {parf }}\left(\mathcal{D}_{\mathcal{P} \#}^{\dagger}\left({ }^{\dagger} T\right)_{\mathbb{Q}}\right)
$$

defined by posing, $\mathbb{D}_{\mathcal{P} \#, T}(\mathcal{G}):=\mathbb{R} \mathcal{H} m_{\left.\mathcal{D}_{\mathcal{P} \#}^{\dagger}{ }^{\dagger} T\right)_{\mathbb{Q}}}\left(\mathcal{G}, \mathcal{D}_{\mathcal{P} \#}^{\dagger}\left({ }^{\dagger} T\right)_{\mathbb{Q}} \otimes_{\mathcal{O}_{\mathcal{P}}} \omega_{\mathcal{P} \#}^{-1}\right)\left[d_{P}\right]$, for any $\mathcal{G} \in D_{\text {parf }}\left(\mathcal{D}_{\mathcal{P} \#}^{\dagger}\left({ }^{\dagger} T\right)_{\mathbb{Q}}\right)$. When $T$ is empty, we remove it from the notation.

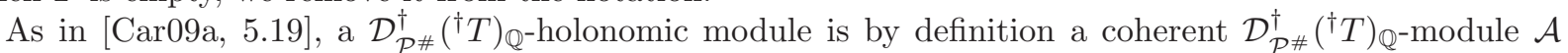
such that, for any $i \neq 0$, we have $\mathcal{H}^{i} \mathbb{D}_{\mathcal{P} \#, T}(\mathcal{A})=0$. For example, let $\mathcal{G}$ be a log-isocrystal on $\mathcal{P}^{\#}$ overconvergent along $T$. Then from [Car09a, 5.17] we have $\mathcal{G} \in D_{\text {parf }}\left(\mathcal{D}_{\mathcal{P} \#}^{\dagger}\left({ }^{\dagger} T\right)_{\mathbb{Q}}\right)$. If we denote by $\mathcal{G}^{\vee}:=\mathcal{H}^{\circ} m_{\mathcal{O}_{\mathcal{P}}\left({ }^{\dagger} T\right)_{\mathbb{Q}}}\left(\mathcal{G}, \mathcal{O}_{\mathcal{P}}\left({ }^{\dagger} T\right)_{\mathbb{Q}}\right)$, then from [Car09a, 5.21] we have the isomorphism

$$
\mathbb{D}_{\mathcal{P} \#, T}(\mathcal{G}) \stackrel{\sim}{\rightarrow} \mathcal{G}^{\vee} .
$$

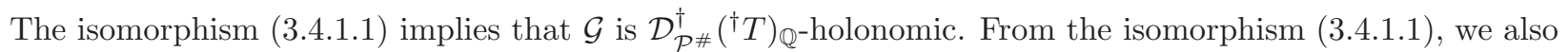
get the following factorizations $\mathbb{D}_{\mathcal{P} \#}: \operatorname{Isoc}\left(\mathcal{P}^{\#} / K\right) \rightarrow \operatorname{Isoc}\left(\mathcal{P}^{\#} / K\right)$ and $\mathbb{D}_{\mathcal{P} \#}: \operatorname{Isoc}^{0}\left(\mathcal{P}^{\#} / K\right) \rightarrow \operatorname{Isoc}^{0}\left(\mathcal{P}^{\#} / K\right)$.

b) If there is no risk of confusion, we put $\mathbb{D}_{T}:=\mathbb{D}_{\mathcal{P} \#, T}$ and we define the functor $\left({ }^{\dagger} T\right): D_{\text {parf }}\left(\mathcal{D}_{\mathcal{P} \# \mathbb{Q}}^{\dagger}\right) \rightarrow$ $D_{\text {parf }}\left(\mathcal{D}_{\mathcal{P} \#}^{\dagger}\left({ }^{\dagger} T\right)_{\mathbb{Q}}\right)$ by setting $\left({ }^{\dagger} T\right)(\mathcal{G}):=\mathcal{D}_{\mathcal{P} \#}^{\dagger}\left({ }^{\dagger} T\right)_{\mathbb{Q}} \otimes_{\mathcal{D}_{\mathcal{P} \# \mathbb{Q}}^{\dagger}} \mathcal{G}$ for any $\mathcal{G} \in D_{\text {parf }}\left(\mathcal{D}_{\mathcal{P} \# \mathbb{Q}}^{\dagger}\right)$. We recall that dual functors commute with the extensions (see [Vir00, I.4.4]), i.e.

$$
\mathbb{D}_{T} \circ\left({ }^{\dagger} T\right) \stackrel{\sim}{\rightarrow}\left({ }^{\dagger} T\right) \circ \mathbb{D}
$$

as functors $D_{\text {parf }}\left(\mathcal{D}_{\mathcal{P} \# \mathbb{Q}}^{\dagger}\right) \rightarrow D_{\text {parf }}\left(\mathcal{D}_{\mathcal{P} \#}^{\dagger}\left({ }^{\dagger} T\right)_{\mathbb{Q}}\right)$.

3.4.2 (Holonomicity, relative duality isomorphism of $\left.i_{1}^{\#}\right)$. Tensoring by $\mathcal{O}_{\mathcal{X}}(\mathfrak{D}):=\mathcal{H}_{o m_{\mathcal{O}_{\mathcal{X}}}}\left(\omega_{\mathcal{X}}, \omega_{\mathcal{X} \#}\right)$, we have the evaluation isomorphism $\omega_{\mathcal{X}} \otimes_{\mathcal{O}_{\mathcal{X}}} \mathcal{O}_{\mathcal{X}}(\mathfrak{D}) \stackrel{\sim}{\rightarrow} \omega_{\mathcal{X} \#}$. Moreover, using the projection isomorphism, since $i_{1}^{*} \mathcal{O}_{\mathcal{X}_{1}}(\mathfrak{D})=\mathcal{O}_{\mathcal{Z}_{1}}\left(\mathfrak{D}_{1}\right)$, we get similarly $i_{1 *} \omega_{\mathcal{Z}_{1}} \otimes_{\mathcal{O}_{\mathcal{X}}} \mathcal{O}_{\mathcal{X}}(\mathfrak{D}) \stackrel{\sim}{\rightarrow} i_{1 *}\left(\omega_{\mathcal{Z}_{1}} \otimes_{\mathcal{O}_{\mathcal{Z}_{1}}} \mathcal{O}_{\mathcal{Z}_{1}}\left(\mathfrak{D}_{1}\right)\right) \stackrel{\sim}{\rightarrow} i_{1 *} \omega_{\mathcal{Z}_{1}^{\#}}$. Hence, by applying the tensor product by $\mathcal{O}_{\mathcal{X}}(\mathfrak{D})$ over $\mathcal{O}_{\mathcal{X}}$ to the well known canonical isomorphism $i_{1 *} \omega_{\mathcal{Z}_{1}} \stackrel{\sim}{\rightarrow}$ $\mathbb{R H o m}_{\mathcal{O}_{\mathcal{X}}}\left(i_{1 *} \mathcal{O}_{\mathcal{Z}_{1}}, \omega_{\mathcal{X}}\right)$ (use the transitivity of the extraordinary inverse image as $\mathcal{O}$-module as defined by Grothendieck in [Har66]), we get $\omega_{\mathcal{Z}_{1}^{\#}} \stackrel{\sim}{\rightarrow} \mathbb{R} \mathcal{H o m}_{\mathcal{O}_{\mathcal{X}}}\left(i_{1 *} \mathcal{O}_{\mathcal{Z}_{1}}, \omega_{\mathcal{X} \#}\right)$. Hence we can copy the construction of [Meb89, I.5.3.2] and then we obtain, for any $\mathcal{G} \in D_{\text {parf }}\left(\mathcal{D}_{\mathcal{P} \#}^{\dagger}\left({ }^{\dagger} T\right)_{\mathbb{Q}}\right)$, the isomorphism

$$
i_{1+}^{\#}\left(\mathbb{D}_{\mathcal{Z}_{1}^{\#}}(\mathcal{G})\right) \stackrel{\sim}{\rightarrow} \mathbb{D}_{\mathcal{X} \#}\left(i_{1+}^{\#}(\mathcal{G})\right),
$$

which is called the relative duality isomorphism. Notice that when the closed immersion is not exact, the isomorphism (3.4.2.1) is not true anymore (e.g. consider the isomorphism (3.4.3.2) which is a kind of twisted relative duality isomorphism). Since the functor $i_{1+}^{\#}$ is exact on the category of coherent $\mathcal{D}_{\mathcal{Z}_{1}^{\#}, \mathbb{Q}^{-}}^{- \text {modules, }}$ the isomorphism (3.4.2.1) implies that if $\mathcal{G}$ is a holonomic $\mathcal{D}_{\mathcal{Z}_{1}^{\#}, \mathbb{Q}^{-m o d u l e}}^{\dagger}$ then $i_{1+}^{\#}(\mathcal{G})$ is $\mathcal{D}_{\mathcal{X}}^{\dagger}, \mathbb{Q}^{- \text {-holonomic. }}$ 
3.4.3. Using $[\mathrm{CT} 12,2.2 .1]$, we get $\mathcal{D}_{\mathcal{X}}^{\dagger}\left({ }^{\dagger} Z\right)_{\mathbb{Q}}=\mathcal{D}_{\mathcal{X}^{b}}^{\dagger}\left({ }^{\dagger} Z\right)_{\mathbb{Q}}$. Hence, the functors $\mathbb{D}_{\mathcal{X}^{b}, Z}$ and $\mathbb{D}_{\mathcal{X}, Z}$ are equal and we can simply denote it by $\mathbb{D}_{Z}$ without risk of confusion. Since $\mathcal{F}, \mathbb{D}_{\mathcal{X}^{b}}(\mathcal{F}) \in \operatorname{Isoc}^{0}\left(\mathcal{X}^{b} / K\right)$ (see 3.4.1.a) for the last one), from $[\mathrm{CT} 12,2.2 .9]$, we get $u_{+}(\mathcal{F}) \stackrel{\sim}{\rightarrow}\left({ }^{\dagger} Z\right)(\mathcal{F})$, and $u_{+}\left(\mathbb{D}_{\mathcal{X}^{b}}(\mathcal{F})\right) \stackrel{\sim}{\rightarrow}\left({ }^{\dagger} Z\right)\left(\mathbb{D}_{\mathcal{X}^{b}}(\mathcal{F})\right)$. Hence, we have the isomorphisms

$$
u_{+} \circ \mathbb{D}_{\mathcal{X}^{b}}(\mathcal{F}) \stackrel{\sim}{\rightarrow}\left({ }^{\dagger} Z\right) \circ \mathbb{D}_{\mathcal{X}^{b}}(\mathcal{F}) \underset{(3.4 .1 .2)}{\stackrel{\sim}{\longrightarrow}} \mathbb{D}_{Z} \circ\left({ }^{\dagger} Z\right)(\mathcal{F}) \stackrel{\sim}{\rightarrow} \mathbb{D}_{Z} \circ u_{+}(\mathcal{F}),
$$

which are the identity over $\mathcal{Y}$. By applying Theorem [Car09a, 5.24.(ii)] to $\mathcal{F}(-\mathcal{Z}) \in \operatorname{Isoc}\left(\mathcal{X}^{b} / K\right)$ (we do not need in this theorem the nilpotence of the residue), since $\mathcal{F}=\mathcal{F}(-\mathcal{Z})(\mathcal{Z})$, we get the isomorphism

$$
u_{!}(\mathcal{F}) \stackrel{\sim}{\longrightarrow} u_{+}(\mathcal{F}(-\mathcal{Z})),
$$

which is the biduality isomorphism over $\mathcal{Y}$. Then we get the isomorphism

$$
j_{!}(\mathcal{E})=\mathbb{D} \circ \mathbb{D}_{Z} \circ u_{+}(\mathcal{F}) \underset{(3.4 .3 .1)}{\stackrel{\sim}{\longrightarrow}} \mathbb{D} \circ u_{+} \circ \mathbb{D}_{\mathcal{X}^{b}}(\mathcal{F})=u_{!}(\mathcal{F}) \underset{(3.4 .3 .2)}{\stackrel{\sim}{\longrightarrow}} u_{+}(\mathcal{F}(-\mathcal{Z})),
$$

which is the biduality isomorphism over $\mathcal{Y}$.

3.4.4. By copying word for word their proof, the results of [Car09a] can be extended from the context of the morphism $u: \mathcal{X}^{b} \rightarrow \mathcal{X}$ to the context of the morphism $u_{1}: \mathcal{X}^{b} \rightarrow \mathcal{X}^{\#}$, i.e. to the case of a morphism which partially forget the log structure. For the reader, we display here these results that we will need and explain briefly how they are checked.

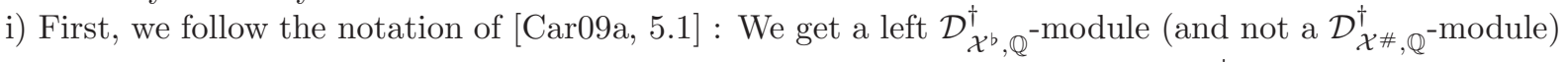

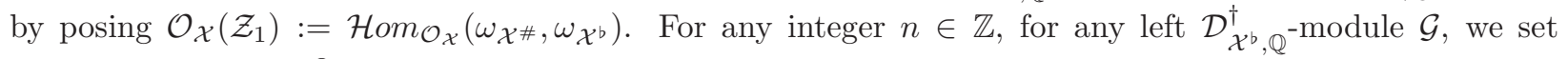
$\mathcal{O}_{\mathcal{X}}\left(n \mathcal{Z}_{1}\right):=\mathcal{O}_{\mathcal{X}}\left(\mathcal{Z}_{1}\right)^{\otimes n}$ ( where $\otimes n$ means that we tensorise $n$ times as $\mathcal{O}_{\mathcal{Z}_{1}}$-module) and $\mathcal{G}\left(n \mathcal{Z}_{1}\right):=\mathcal{G} \otimes_{\mathcal{O}_{\mathcal{X}}}$

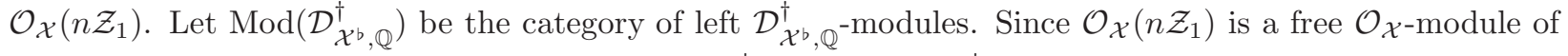
rank one, then the induced functors $\left(n \mathcal{Z}_{1}\right): \operatorname{Mod}\left(\mathcal{D}_{\mathcal{X}^{b}, \mathbb{Q}}^{\dagger}\right) \rightarrow \operatorname{Mod}\left(\mathcal{D}_{\mathcal{X}^{b}, \mathbb{Q}}^{\dagger}\right)$, given by $\mathcal{G} \mapsto \mathcal{G}\left(n \mathcal{Z}_{1}\right)$, is exact. We get the canonical isomorphism $\omega_{\mathcal{X}^{\mathrm{b}}} \otimes_{\mathcal{O}_{\mathcal{X}}}\left(\mathcal{G} \otimes_{\mathcal{O}_{\mathcal{X}}} \omega_{\mathcal{X}^{\#}}^{-1} \stackrel{\sim}{\rightarrow} \mathcal{G}\left(\mathcal{Z}_{1}\right)\right.$ (as in [Car09a, 5.2.4]). Hence, as in [Car09a, 5.8], we get a $\left(\mathcal{D}_{\mathcal{X}^{\#}}^{\dagger}, \mathcal{D}_{\mathcal{X}^{b}}^{\dagger}\right)$-bimodule by setting $\mathcal{D}_{\mathcal{X}^{\#} \leftarrow \mathcal{X}^{b}}^{\dagger}:=\omega_{\mathcal{X}^{b}}{ }^{\mathrm{d}} \otimes_{\mathcal{O}_{\mathcal{X}}}\left(\mathcal{D}_{\mathcal{X}^{\#}}^{\dagger} \otimes_{\mathcal{O}_{\mathcal{X}}} \omega_{\mathcal{X}^{\#}}^{-1}\right) \stackrel{\sim}{\rightarrow} \mathcal{D}_{\mathcal{X}^{\#}}^{\dagger} \otimes_{\mathcal{O}_{\mathcal{X}}} \mathcal{O}_{\mathcal{X}}\left(\mathcal{Z}_{1}\right)$,

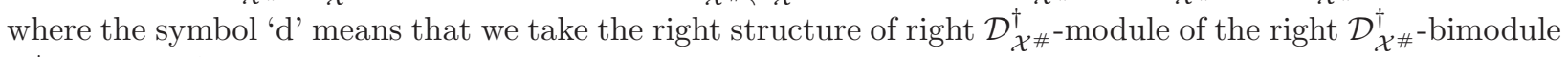
$\mathcal{D}_{\mathcal{X} \#}^{\dagger} \otimes_{\mathcal{O}_{\mathcal{X}}} \omega_{\mathcal{X} \#}^{-1}$ and the isomorphism is induced by the evaluation map (in fact, in [Car09a, 5.8] we had chosen

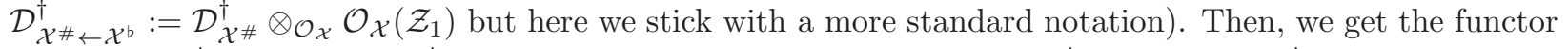
$u_{1+}: D_{\text {coh }}^{\mathrm{b}}\left(\mathcal{D}_{\mathcal{X}^{b}, \mathbb{Q}}^{\dagger}\right) \rightarrow D_{\text {coh }}^{\mathrm{b}}\left(\mathcal{D}_{\mathcal{X}^{\#}, \mathbb{Q}}^{\dagger}\right)$ defined by posing, for any $\mathcal{G} \in D_{\text {coh }}^{\mathrm{b}}\left(\mathcal{D}_{\mathcal{X}^{b}, \mathbb{Q}}^{\dagger}\right), u_{1+}:=\mathcal{D}_{\mathcal{X}^{\#} \leftarrow \mathcal{X}^{b}, \mathbb{Q}}^{\dagger} \otimes_{\mathcal{D}_{\mathcal{X}^{b}, \mathbb{Q}}^{\dagger}}^{\mathbb{L}} \mathcal{G}$.

ii) Similarly than $\left[\right.$ Car09a, 5.12], we put $u_{1 !}:=\mathbb{D}_{\mathcal{X}^{\#}} \circ u_{1+} \circ \mathbb{D}_{\mathcal{X}^{b}}: D_{\operatorname{coh}}^{\mathrm{b}}\left(\mathcal{D}_{\mathcal{X}^{b}, \mathbb{Q}}^{\dagger}\right) \rightarrow D_{\operatorname{coh}}^{\mathrm{b}}\left(\mathcal{D}_{\mathcal{X}^{\#}, \mathbb{Q}}^{\dagger}\right)$. Using some transposition isomorphims for $\mathcal{X}^{\#}$ and $\mathcal{X}^{b}$ (i.e. [Car09a, 1.18 and 1.19]), we check as for Proposition [Car09a, 5.14] (see the notation [Car09a, 5.3]) the isomorphism

$$
u_{1 !}(\mathcal{G}) \stackrel{\sim}{\rightarrow} \mathcal{D}_{\mathcal{X} \#, \mathbb{Q}}^{\dagger} \otimes_{\mathcal{D}_{\mathcal{X}^{b}, \mathbb{Q}}^{\dagger}}^{\mathbb{L}} \mathcal{G}
$$

for any $\mathcal{G} \in D_{\text {coh }}^{\mathrm{b}}\left(\mathcal{D}_{\mathcal{X}^{b}, \mathbb{Q}}^{\dagger}\right)$. Moreover, as in [Car09a, 5.16], we have the canonical isomorphism

$$
\iota_{1, \mathcal{G}}: u_{1 !}(\mathcal{G}) \stackrel{\sim}{\rightarrow} u_{1+}\left(\mathcal{G}\left(-\mathcal{Z}_{1}\right)\right)
$$

which is the biduality isomorphism outside $Z_{1}$. The main point of the proof of the isomorphism (3.4.4.2) is to check the canonical isomorphism of right $\mathcal{D}_{\mathcal{X}^{b}}^{(0)}$-modules of the form $\left(\omega_{\mathcal{X}} \otimes_{\mathcal{O}_{\mathcal{X}}} \mathcal{G}^{b}\right) \otimes_{\mathcal{D}_{\mathcal{X}^{b}}^{(0)}} \mathcal{G}^{\#} \stackrel{\sim}{\rightarrow}\left(\omega_{\mathcal{X} \#} \otimes_{\mathcal{O}_{\mathcal{X}}}\right.$ $\left.\mathcal{G}^{\#}\right) \otimes_{\mathcal{D}_{\mathcal{X}^{b}}^{(0)}} \mathcal{G}^{b}$, for any $\mathcal{D}_{\mathcal{X}^{b}}^{(0)}$-bimodule $\mathcal{G}^{\#}$ and any left $\mathcal{D}_{\mathcal{X}^{\#}}^{(0)}$-module $\mathcal{G}^{b}$. The check of this last isomorphism is the same as that of [Car09a, 3.7.1] (more precisely, every results of [Car09a, 3] can be extended in this relative context : replace everywhere respectively $\mathcal{X}$ by $\mathcal{X}^{\#}$ and $\mathcal{X}^{\#}$ by $\left.\mathcal{X}^{b}\right)$. 
iii) Let $\mathcal{G} \in \operatorname{Isoc}\left(\mathcal{X}^{b} / K\right)$. As in [Car09a, 2.6], we define the following first Spencer sequence associated with $\mathcal{G}$ :

$$
0 \rightarrow \mathcal{D}_{\mathcal{X}^{b}, \mathbb{Q}} \otimes_{\mathcal{O}_{\mathcal{X}}} \wedge{ }^{d} \mathcal{T}_{\mathcal{X}^{b}} \otimes_{\mathcal{O}_{\mathcal{X}}} \mathcal{G} \rightarrow \cdots \rightarrow \mathcal{D}_{\mathcal{X}^{b}, \mathbb{Q}} \otimes_{\mathcal{O}_{\mathcal{X}}} \wedge{ }^{1} \mathcal{T}_{\mathcal{X}^{b}} \otimes_{\mathcal{O}_{\mathcal{X}}} \mathcal{G} \rightarrow \mathcal{D}_{\mathcal{X}^{b}, \mathbb{Q}} \otimes_{\mathcal{O}_{\mathcal{X}}} \mathcal{G} \rightarrow \mathcal{G} \rightarrow 0
$$

where $\mathcal{T}_{\mathcal{X}^{b}}$ is the tangent space of $\mathcal{X}^{b} / \mathcal{V}$ (recall that $\mathcal{D}_{\mathcal{X}^{b}, \mathbb{Q}}=\mathcal{D}_{\mathcal{X}^{b}}^{(0)} \otimes_{\mathcal{O}_{\mathcal{X}}} \mathcal{O}_{\mathcal{X}, \mathbb{Q}}$ ). We denote by $S p_{\mathcal{D}_{\mathcal{X}^{b}, \mathbb{Q}}}(\mathcal{G})$ the sequence (3.4.4.3). As in [Car09a, 2.8], we check that the sequence $S p_{\mathcal{D}_{\mathcal{X}^{b}, \mathbb{Q}}^{\bullet}}(\mathcal{G})$ is exact. Moreover,

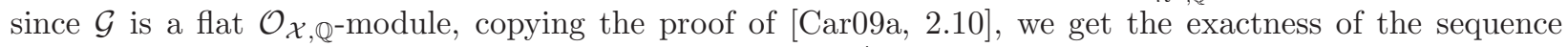
$\mathcal{D}_{\mathcal{X} \#, \mathbb{Q}} \otimes_{\mathcal{D}_{\mathcal{X}^{b}, \mathbb{Q}}} S p_{\mathcal{D}_{\mathcal{X}^{b}, \mathbb{Q}}}(\mathcal{G})$. Since the extension $\mathcal{D}_{\mathcal{X} \#, \mathbb{Q}} \rightarrow \mathcal{D}_{\mathcal{X} \#, \mathbb{Q}}^{\dagger}$ is flat, we get the exactness of the sequence $\mathcal{D}_{\mathcal{X}^{\#, Q} \mathbb{Q}}^{\dagger} \otimes_{\mathcal{D}_{\mathcal{X}^{b}, \mathbb{Q}}} S p_{\mathcal{D}_{\mathcal{X}^{b}, \mathbb{Q}}}^{\bullet}(\mathcal{G})$. Since $S p_{\mathcal{D}_{\mathcal{X}^{b}, \mathbb{Q}}}^{\bullet}(\mathcal{G})$ gives a flat resolution of $\mathcal{G}$ by left $\mathcal{D}_{\mathcal{X}^{b}, \mathbb{Q}^{-m o d u l e s, ~ t h i s ~ i m p l i e s ~}}$ that the canonical morphism $\mathcal{D}_{\mathcal{X} \#, \mathbb{Q}}^{\dagger} \otimes_{\mathcal{D}_{\mathcal{X}^{b}, \mathbb{Q}}^{\mathbb{L}}}^{\mathbb{G}} \rightarrow \mathcal{D}_{\mathcal{X}^{\#, \mathbb{Q}}}^{\dagger} \otimes_{\mathcal{D}_{\mathcal{X}^{b}, \mathbb{Q}}} \mathcal{G}$ is an isomorphism. From [Car09a, 4.14], the canonical morphism $\mathcal{G} \rightarrow \mathcal{D}_{\mathcal{X}^{b}, \mathbb{Q}}^{\dagger} \otimes_{\mathcal{D}_{\mathcal{X}^{b}, \mathbb{Q}}} \mathcal{G} \stackrel{\sim}{\sim} \mathcal{D}_{\mathcal{X}^{b}, \mathbb{Q}}^{\dagger} \otimes_{\mathcal{D}_{\mathcal{X}^{b}, \mathbb{Q}}}^{\mathbb{L}} \mathcal{G}$ is an isomorphism (the last isomorphism comes from the flatness of $\left.\mathcal{D}_{\mathcal{X}^{b}, \mathbb{Q}} \rightarrow \mathcal{D}_{\mathcal{X}^{b}, \mathbb{Q}}^{\dagger}\right)$. This yields the last canonical isomorphism: $u_{1 !}(\mathcal{G}) \underset{(3.4 .4 .1)}{\stackrel{\sim}{\longrightarrow}}$ $\mathcal{D}_{\mathcal{X} \#, \mathbb{Q}}^{\dagger} \otimes_{\mathcal{D}_{\mathcal{X}^{b}, \mathbb{Q}}^{\dagger}}^{\mathbb{L}} \mathcal{G} \stackrel{\sim}{\rightarrow} \mathcal{D}_{\mathcal{X} \#, \mathbb{Q}}^{\dagger} \otimes_{\mathcal{D}_{\mathcal{X}^{b}, \mathbb{Q}}^{\dagger}} \mathcal{G}$. With (3.4.4.2), this implies

$$
u_{1+}(\mathcal{G}) \stackrel{\sim}{\rightarrow} \mathcal{D}_{\mathcal{X} \#, \mathbb{Q}}^{\dagger} \otimes_{\mathcal{D}_{\mathcal{X}^{b}, \mathbb{Q}}^{\dagger}} \mathcal{G}\left(\mathcal{Z}_{1}\right)
$$

iv) Finally, from $[\operatorname{Car} 12 b, 3.5 .6 .2]$, for any $\mathcal{G} \in \operatorname{Isoc}^{0}\left(\mathcal{X}^{b} / K\right)$ (not only $\operatorname{Isoc}\left(\mathcal{X}^{b} / K\right)$ ), we have the isomorphism

$$
\rho_{1, \mathcal{G}}: u_{1+}(\mathcal{G}) \stackrel{\sim}{\rightarrow}\left({ }^{\dagger} Z_{1}\right)(\mathcal{G}),
$$

which is a generalization of $[\mathrm{CT} 12,2.2 .9]$ that we will need.

3.4.5. We denote by $\alpha_{1, \mathcal{F}}: \mathcal{F}\left(-\mathcal{Z}_{1}\right) \hookrightarrow \mathcal{F}$ the canonical monomorphism of $\operatorname{Isoc}\left(\mathcal{X}^{b} / K\right)$. From (3.4.4.4), for any $\mathcal{G} \in \operatorname{Isoc}\left(\mathcal{X}^{b} / K\right), \mathcal{H}^{i} u_{1+}(\mathcal{G})=0(i \neq 0)$. Thus, $\alpha_{1, \mathcal{F}}$ induces the homomorphism of coherent $\mathcal{D}_{\mathcal{X} \#, \mathbb{Q}^{-}}^{\dagger}$ modules $\beta_{1, \mathcal{F}}:=u_{1+}\left(\alpha_{1, \mathcal{F}}\right): u_{1+}\left(\mathcal{F}\left(-\mathcal{Z}_{1}\right)\right) \rightarrow u_{1+}(\mathcal{F})$. In fact, with (3.4.4.2) and (3.4.1.1), we check that

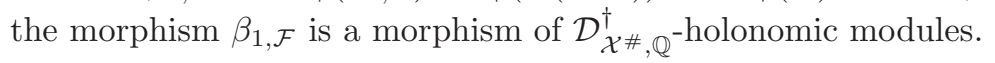

3.4.6 (Cone of $\left.\beta_{1, \mathcal{F}}\right)$. Since the module $\mathcal{F}\left(-\mathcal{Z}_{1}\right) \in \operatorname{Isoc}\left(\mathcal{X}^{b} / K\right)$ and its exponents and their differences are non Liouville, then from [Car12b, 3.5.5.1], $i_{1}^{\# !}\left(u_{1+}\left(\mathcal{F}\left(-\mathcal{Z}_{1}\right)\right)\right)$ is isomorphic to a complex of objects in $\operatorname{Isoc}\left(\mathcal{Z}_{1} \# / K\right)$ and in particular belongs to $D_{\text {coh }}^{\mathrm{b}}\left(\mathcal{D}_{\mathcal{Z}_{1}^{\#}, \mathbb{Q}}^{\dagger}\right)$. In our special context, since the residues of $\mathcal{F}\left(-\mathcal{Z}_{1}\right)$ induces by $t_{i} \partial_{i}$ for $i \geq 2$ are nilpotent, then so are that of the isocrystals of the complex $i_{1}^{\prime \# !}\left(u_{1+}\left(\mathcal{F}\left(-\mathcal{Z}_{1}\right)\right)\right)$ (see [CT12, 1.1.22] which is the main argument of the check of [Car12b, 3.5.5.1]). Moreover, with [Car12b, 3.5.5.2], we have the exact triangle of $D_{\mathrm{coh}}^{\mathrm{b}}\left(\mathcal{D}_{\mathcal{X}}^{\dagger}, \mathbb{Q}\right)$ :

$$
i_{1+}^{\#} i_{1}^{\# !}\left(u_{1+}\left(\mathcal{F}\left(-\mathcal{Z}_{1}\right)\right)\right) \rightarrow u_{1+}\left(\mathcal{F}\left(-\mathcal{Z}_{1}\right)\right) \rightarrow\left({ }^{\dagger} Z_{1}\right) \circ u_{1+}\left(\mathcal{F}\left(-\mathcal{Z}_{1}\right)\right) \rightarrow+1
$$

Since $\mathcal{D}_{\mathcal{X}^{\#}}^{\dagger}\left({ }^{\dagger} Z_{1}\right)_{\mathbb{Q}}=\mathcal{D}_{\mathcal{X}^{b}}^{\dagger}\left({ }^{\dagger} Z_{1}\right)_{\mathbb{Q}}$ (use $\left.[\mathrm{CT} 12,2.2 .1]\right)$, we get :

$$
\left({ }^{\dagger} Z_{1}\right) \circ u_{1+}\left(\mathcal{F}\left(-\mathcal{Z}_{1}\right)\right):=\mathcal{D}_{\mathcal{X} \#}^{\dagger}\left({ }^{\dagger} Z_{1}\right)_{\mathbb{Q}} \otimes_{\mathcal{D}_{\mathcal{X} \#, \mathbb{Q}}^{\dagger}} u_{1+}\left(\mathcal{F}\left(-\mathcal{Z}_{1}\right)\right) \underset{(3.4 .4 .4)}{\stackrel{\sim}{\longrightarrow}} \mathcal{D}_{\mathcal{X}^{b}}^{\dagger}\left({ }^{\dagger} Z_{1}\right)_{\mathbb{Q}} \otimes_{\mathcal{D}_{\mathcal{X}^{b}, \mathbb{Q}}^{\dagger}} \mathcal{F}=:\left({ }^{\dagger} Z_{1}\right)(\mathcal{F}) .
$$

Since $\mathcal{F} \in \operatorname{Isoc}^{0}\left(\mathcal{X}^{b} / K\right)$, from (3.4.4.5), we have $\rho_{1, \mathcal{F}}: u_{1+}(\mathcal{F}) \stackrel{\sim}{\rightarrow}\left({ }^{\dagger} Z_{1}\right)(\mathcal{F})$. As in (3.4.6.2), this yields that the canonical morphism $u_{1+}(\mathcal{F}) \rightarrow\left({ }^{\dagger} Z_{1}\right) \circ u_{1+}(\mathcal{F})$ is an isomorphism. Hence, from (3.4.6.1), we get the exact triangle of $D_{\mathrm{coh}}^{\mathrm{b}}\left(\mathcal{D}_{\mathcal{X}^{\#}, \mathbb{Q}}^{\dagger}\right)$ :

$$
i_{1+}^{\#} i_{1}^{\# !}\left(u_{1+}\left(\mathcal{F}\left(-\mathcal{Z}_{1}\right)\right)\right) \rightarrow u_{1+}\left(\mathcal{F}\left(-\mathcal{Z}_{1}\right)\right) \stackrel{\beta_{1, \mathcal{F}}}{\longrightarrow} u_{1+}(\mathcal{F}) \rightarrow+1
$$


Since $\operatorname{Isoc}^{0}\left(\mathcal{Z}_{1}^{\#} / K\right)$ is an abelian category and since $i_{1}^{\# !}\left(u_{1+}\left(\mathcal{F}\left(-\mathcal{Z}_{1}\right)\right)\right)$ is isomorphic to a complex of $\operatorname{Isoc}^{0}\left(\mathcal{Z}_{1}^{\#} / K\right)$, from (3.4.6.3), we get that $i_{1}^{\# !}\left(\operatorname{ker}\left(\beta_{1, \mathcal{F}}\right)\right)$ and $i_{1}^{\# !}\left(\operatorname{coker}\left(\beta_{1, \mathcal{F}}\right)\right)$ are objects of the abelian category $\operatorname{Isoc}^{0}\left(\mathcal{Z}_{1}^{\#} / K\right)$.

Since $i_{1+}^{\#}$ preserves the holonomicity (see 3.4.2), we get from (3.4.6.3) the long exact sequence of holonomic $\mathcal{D}_{\mathcal{X} \#, \mathbb{Q}^{-m o d u l e s}}^{\dagger}$

$$
0 \rightarrow \operatorname{ker}\left(\beta_{1, \mathcal{F}}\right) \rightarrow u_{1+}\left(\mathcal{F}\left(-\mathcal{Z}_{1}\right)\right) \stackrel{\beta_{1, \mathcal{F}}}{\longrightarrow} u_{1+}(\mathcal{F}) \rightarrow \operatorname{coker}\left(\beta_{1, \mathcal{F}}\right) \rightarrow 0 .
$$

Using the definition of holonomicity, we check that the image of $\beta_{1, \mathcal{F}}$ is also holonomic. (Remark that this is

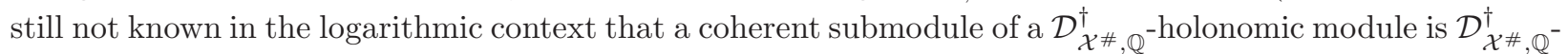
holonomic (but this is probably true). So a priori we do need the holonomicity of $\operatorname{ker}\left(\beta_{1, \mathcal{F}}\right)$ and $\operatorname{coker}\left(\beta_{1, \mathcal{F}}\right)$ to check the holonomicity of the image of $\beta_{1, \mathcal{F}}$.) Hence, by applying the functor $\mathcal{H}^{0} \mathbb{D}_{\mathcal{X}}$ to the long exact sequence (3.4.6.4), we get another long exact sequence which can be translated by the isomorphisms

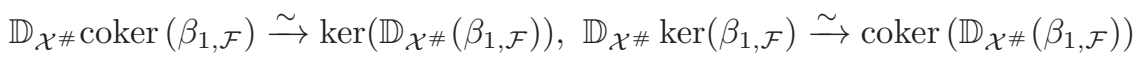

where we remove $\mathcal{H}^{0}$ in the notation since modules are holonomic.

3.4.7. i) As $g_{1}^{\#}: \mathcal{X}^{\prime \#} \rightarrow \mathcal{Z}_{1}^{\#}$ is $\log$ smooth of relative dimension 1, using [Ogu, IV.3.2.3], we check that $\Omega_{\mathcal{X}^{\prime \prime} / \mathcal{Z}_{1}^{\#}}^{1}=\omega_{\mathcal{X}^{\prime} \# / \mathcal{Z}_{1}^{\#}} \stackrel{\sim}{\rightarrow} \omega_{\mathcal{X}^{\prime \prime \#}} \otimes_{g_{1}^{-1} \mathcal{O}_{\mathcal{Z}_{1}}} g_{1}^{-1}\left(\omega_{\mathcal{Z}_{1}^{\#}}^{-1}\right)$. We denote by $\delta_{\mathcal{X}^{\prime \prime}}: \omega_{\mathcal{X}^{\prime} \#} \otimes_{\mathcal{O}_{\mathcal{X}^{\prime}}} \widehat{\mathcal{D}}_{\mathcal{X}^{\prime \prime \#}}^{(0)} \stackrel{\sim}{\rightarrow} \omega_{\mathcal{X}^{\prime} \#} \otimes_{\mathcal{O}_{\mathcal{X}^{\prime}}} \widehat{\mathcal{D}}_{\mathcal{X}^{\prime \prime}}^{(0)}$ the canonical involution which exchanges both right $\widehat{\mathcal{D}}_{\mathcal{X}^{\prime \prime \#}}^{(0)}$-module structures (this comes from [Car09a, 1.19] by completion) : $\omega_{\mathcal{X}^{\prime} \#} \otimes_{\mathcal{O}_{\mathcal{X}^{\prime}}} \widehat{\mathcal{D}}_{\mathcal{X}^{\prime \prime} \#}^{(0)} \stackrel{\sim}{\rightarrow} \omega_{\mathcal{X}^{\prime} \#} \otimes_{\mathcal{O}_{\mathcal{X}^{\prime}}} \widehat{\mathcal{D}}_{\mathcal{X}^{\prime \prime} \#}^{(0)}$. The canonical morphism of left $\widehat{\mathcal{D}}_{\mathcal{X}^{\prime} \# \text {-module of the }}^{(0)}$ form $\widehat{\mathcal{D}}_{\mathcal{X}^{\prime \#}}^{(0)} \rightarrow g_{1}^{*}\left(\widehat{\mathcal{D}}_{\mathcal{Z}_{1}^{\#}}^{(0)}\right)$ induces the morphism of left $\left(\widehat{\mathcal{D}}_{\mathcal{X}^{\prime \#}}^{(0)}, g_{1}^{-1} \widehat{\mathcal{D}}_{\mathcal{Z}_{1}^{\#}}^{(0)}\right)$-bimodules of the form $\widehat{\mathcal{D}}_{\mathcal{X}^{\prime \prime}}^{(0)} \otimes_{g_{1}^{-1} \mathcal{O}_{\mathcal{Z}_{1}}}$ $g_{1}^{-1}\left(\omega_{\mathcal{Z}_{1}^{\#}}^{-1}\right) \rightarrow g_{1 \mathrm{~g}}^{*}\left(\widehat{\mathcal{D}}_{\mathcal{Z}_{1}^{\#}}^{(0)} \otimes_{\mathcal{O}_{\mathcal{Z}_{1}}} \omega_{\mathcal{Z}_{1}^{\#}}^{-1}\right)$, where the symbol 'g' means that we choose the left structure of right $\widehat{\mathcal{D}}_{\mathcal{Z}_{1}^{\#}}^{(0)}$-module of $\widehat{\mathcal{D}}_{\mathcal{Z}_{1}^{\#}}^{(0)} \otimes_{\mathcal{O}_{\mathcal{Z}_{1}}} \omega_{\mathcal{Z}_{1}^{\#}}^{-1}$. We denote by $\beta_{\mathcal{Z}_{1}^{\#}}: \widehat{\mathcal{D}}_{\mathcal{Z}_{1}^{\#}}^{(0)} \otimes_{\mathcal{O}_{Z_{1}}} \omega_{\mathcal{Z}_{1}^{\#}}^{-1} \stackrel{\sim}{\rightarrow} \widehat{\mathcal{D}}_{\mathcal{Z}_{1}^{\#}}^{(0)} \otimes_{\mathcal{O}_{Z_{1}}} \omega_{\mathcal{Z}_{1}^{\#}}^{-1}$ the involution isomorphism which exchanges both left $\widehat{\mathcal{D}}_{\mathcal{X}^{\prime \prime}}^{(0)}$-module structures. Applying the functor $\omega_{\mathcal{X}^{\prime \prime}} \otimes_{\mathcal{O}_{\mathcal{X}^{\prime}}}-$ to these morphisms of left $\left(\widehat{\mathcal{D}}_{\mathcal{X}^{\prime \#}}^{(0)}, g_{1}^{-1} \widehat{\mathcal{D}}_{\mathcal{Z}_{1}^{\#}}^{(0)}\right)$-bimodules we get the morphism of $\left(g_{1}^{-1} \widehat{\mathcal{D}}_{\mathcal{Z}_{1}^{\#}}^{(0)}, \widehat{\mathcal{D}}_{\mathcal{X}^{\prime \#}}^{(0)}\right)$-bimodules of the form:

$\omega_{\mathcal{X}^{\prime \prime \#}} \otimes_{\mathcal{O}_{\mathcal{X}^{\prime}}}\left(\widehat{\mathcal{D}}_{\mathcal{X}^{\prime \prime}}^{(0)} \otimes_{g_{1}^{-1} \mathcal{O}_{\mathcal{Z}_{1}}} g_{1}^{-1}\left(\omega_{\mathcal{Z}_{1}^{\#}}^{-1}\right)\right) \rightarrow \omega_{\mathcal{X}^{\prime \prime}} \otimes_{\mathcal{O}_{\mathcal{X}^{\prime}}} g_{1 \mathrm{~g}}^{*}\left(\widehat{\mathcal{D}}_{\mathcal{Z}_{1}^{\#}}^{(0)} \otimes_{\mathcal{O}_{\mathcal{Z}_{1}}} \omega_{\mathcal{Z}_{1}^{\#}}^{-1}\right) \underset{\beta_{\mathcal{Z}_{1}^{\#}}}{\stackrel{\sim}{\longrightarrow}} g_{1 \mathrm{~d}}^{*}\left(\widehat{\mathcal{D}}_{\mathcal{Z}_{1}^{\#}}^{(0)} \otimes_{\mathcal{O}_{\mathcal{Z}_{1}}} \omega_{\mathcal{Z}_{1}^{\#}}^{-1}\right) \otimes \mathcal{O}_{\mathcal{X}^{\prime}} \omega_{\mathcal{X}^{\prime} \#}$,

where the symbol 'd' means that we choose the right structure of right $\widehat{\mathcal{D}}_{\mathcal{Z}_{1}^{\#}}^{(0)}$-module of $\widehat{\mathcal{D}}_{\mathcal{Z}_{1}^{\#}}^{(0)} \otimes_{\mathcal{O}_{\mathcal{Z}_{1}}} \omega_{\mathcal{Z}_{1}^{\#}}^{-1}$. Hence, by composing with the canonical involution we get

$$
\Omega_{\mathcal{X}^{\prime} \# / \mathcal{Z}_{1}^{\#}}^{1} \otimes_{\mathcal{O}_{\mathcal{X}^{\prime}}} \widehat{\mathcal{D}}_{\mathcal{X}^{\prime \prime \#}}^{(0)} \stackrel{\sim}{\rightarrow} g_{1}^{-1} \omega_{\mathcal{Z}_{1}^{\#}}^{-1} \otimes_{g_{1}^{-1} \mathcal{O}_{\mathcal{Z}_{1}}}\left(\omega_{\mathcal{X}^{\prime \prime}} \otimes_{\mathcal{O}_{\mathcal{X}^{\prime}}} \widehat{\mathcal{D}}_{\mathcal{X}^{\prime} \#}^{(0)}\right) \underset{\delta_{\mathcal{X}^{\prime} \#} \otimes i d}{\sim}\left(\omega_{\mathcal{X}^{\prime} \#}^{\sim} \otimes_{\mathcal{O}_{\mathcal{X}^{\prime}}} \widehat{\mathcal{D}}_{\mathcal{X}^{\prime} \#}^{(0)}\right) \otimes_{g_{1}^{-1} \mathcal{O}_{\mathcal{Z}_{1}}} g_{1}^{-1} \omega_{\mathcal{Z}_{1}^{\#}}^{-1},
$$

where the canonical right $\widehat{\mathcal{D}}_{\mathcal{X}^{\prime \prime \#}}^{(0)}$-module structure on $\Omega_{\mathcal{X}^{\prime \#} / \mathcal{Z}_{1}^{\#}}^{1} \otimes_{\mathcal{O}_{\mathcal{X}^{\prime}}} \widehat{\mathcal{D}}_{\mathcal{X}^{\prime \prime \#}}^{(0)}$ is the multiplication at the right on $\widehat{\mathcal{D}}_{\mathcal{X}^{\prime \prime}}^{(0)}$ in the tensor product. As in [Mon02, 5.1.1], which can be extended to the relative case, (see also [Ber02, 2.4.6.2] for the non logarithmic relative case), we check that the canonical morphism of right $\widehat{\mathcal{D}}_{\mathcal{X}^{\prime} \# \text {-modules }}^{(0)}$

$$
\Omega_{\mathcal{X}^{\prime \#} / \mathcal{Z}_{1}^{\#}}^{1} \otimes_{\mathcal{O}_{\mathcal{X}^{\prime}}} \widehat{\mathcal{D}}_{\mathcal{X}^{\prime \prime \#}}^{(0)} \rightarrow g_{1 \mathrm{~d}}^{*}\left(\widehat{\mathcal{D}}_{\mathcal{Z}_{1}^{\#}}^{(0)} \otimes_{\mathcal{O}_{\mathcal{Z}_{1}}} \omega_{\mathcal{Z}_{1}^{\#}}^{-1}\right) \otimes_{\mathcal{O}_{\mathcal{X}^{\prime}}} \omega_{\mathcal{X}^{\prime} \#}=: \widehat{\mathcal{D}}_{\mathcal{Z}_{1}^{\#} \leftarrow \mathcal{X}^{\prime \#}}^{(0)}
$$

which is the composition of (3.4.7.2) with (3.4.7.1), induces the exact sequence of right $\widehat{\mathcal{D}}_{\mathcal{X}^{\prime} \# \text {-modules }}^{(0)}$

$$
0 \rightarrow \widehat{\mathcal{D}}_{\mathcal{X}^{\prime} \#}^{(0)} \rightarrow \Omega_{\mathcal{X}^{\prime \#} / \mathcal{Z}_{1}^{\#}}^{1} \otimes_{\mathcal{O}_{\mathcal{X}^{\prime}}} \widehat{\mathcal{D}}_{\mathcal{X}^{\prime \#}}^{(0)} \rightarrow \widehat{\mathcal{D}}_{\mathcal{Z}_{1}^{\#} \leftarrow \mathcal{X}^{\prime} \#}^{(0)} \rightarrow 0
$$


where the first part of the exact sequence is the relative to $g_{1}^{\#}$ de Rham complex of $\widehat{\mathcal{D}}_{\mathcal{X}^{\prime} \#}^{(0)}$. As for [Ber02, 4.3.3.(ii)] (to deduce the right case from the left case, we use the equivalences [Car09a, 1.16]), since $g_{1}^{\#}$ is $\log$ smooth, then $\widehat{\mathcal{D}}_{\mathcal{Z}_{1}^{\#} \leftarrow \mathcal{X}^{\prime \#, \mathbb{Q}}}^{(0)} \otimes_{\widehat{\mathcal{D}}_{\mathcal{X}^{\prime} \#, \mathbb{Q}}^{(0)}} \mathcal{D}_{\mathcal{X}^{\prime} \#, \mathbb{Q}}^{\dagger} \stackrel{\sim}{\rightarrow} \mathcal{D}_{\mathcal{Z}_{1}^{\#} \leftarrow \mathcal{X}^{\prime} \#, \mathbb{Q}}^{\dagger}:=\omega_{\mathcal{X}^{\prime} \#} \otimes_{\mathcal{O}_{\mathcal{X}^{\prime}}} g_{1 \mathrm{~d}}^{*}\left(\mathcal{D}_{\mathcal{Z}_{1}^{\#}, \mathbb{Q}}^{\dagger} \otimes_{\mathcal{O}_{\mathcal{Z}_{1}}} \omega_{\mathcal{Z}_{1}^{\#}}^{-1}\right)$. Hence, since $g_{1 *}$ is exact, since the extension $\widehat{\mathcal{D}}_{\mathcal{X}^{\prime \#}}^{(0)} \rightarrow \mathcal{D}_{\mathcal{X}^{\prime \prime}, \mathbb{Q}}^{\dagger}$ is flat, using the exactness of (3.4.7.4) we get for any coherent left $\mathcal{D}_{\mathcal{X}^{\prime \prime}, \mathbb{Q}}^{\dagger}$-module $\mathcal{G}^{\#}$

$$
g_{1+}^{\#}\left(\mathcal{G}^{\#}\right):=\mathbb{R} g_{1 *}\left(\mathcal{D}_{\mathcal{Z}_{1}^{\#} \leftarrow \mathcal{X}^{\prime \prime}, \mathbb{Q}}^{\dagger} \otimes_{\mathcal{D}_{\mathcal{X}^{\prime} \#, \mathbb{Q}}^{\dagger}}^{\mathbb{L}} \mathcal{G}^{\#}\right) \stackrel{\sim}{\rightarrow} g_{1 *}\left[\mathcal{G}^{\#} \rightarrow \Omega_{\mathcal{X}^{\prime \#} / \mathcal{Z}_{1}^{\#}}^{1} \otimes_{\mathcal{O}_{\mathcal{X}^{\prime}}} \mathcal{G}^{\#}\right][1]
$$

where $\left[\mathcal{G}^{\#} \rightarrow \Omega_{\mathcal{X}^{\prime \#} / \mathcal{Z}_{1}^{\#}}^{1} \otimes_{\mathcal{O}_{\mathcal{X}^{\prime}}} \mathcal{G}^{\#}\right][1]$ means that the position of $\mathcal{G}^{\#}$ is -1 .

ii) Similarly, for any coherent left $\mathcal{D}_{\mathcal{X}^{\prime \prime}, \mathbb{Q}^{-m o d u l e}}^{\dagger} \mathcal{G}^{b}$, we check the isomorphism

$$
g_{1+}^{b}\left(\mathcal{G}^{b}\right):=\mathbb{R} g_{1 *}\left(\mathcal{D}_{\mathcal{Z}_{1}^{\#} \leftarrow \mathcal{X}^{\prime b}, \mathbb{Q}}^{\dagger} \otimes_{\mathcal{D}_{\mathcal{X}^{\prime b}, \mathbb{Q}}^{\dagger}}^{\mathbb{L}} \mathcal{G}^{b}\right) \stackrel{\sim}{\rightarrow} g_{1 *}\left[\mathcal{G}^{b} \rightarrow \Omega_{\mathcal{X}^{\prime b} / \mathcal{Z}_{1}^{\#}}^{1} \otimes_{\mathcal{O}_{\mathcal{X}^{\prime}}} \mathcal{G}^{b}\right][1]
$$

By identifying $\Omega_{\mathcal{X}^{\prime \#} / \mathcal{Z}_{1}^{\#}}^{1}$ (which is free with $d t_{1}$ as a basis) (resp. $\Omega_{\mathcal{X}^{\prime b} / \mathcal{Z}_{1}^{\#}}^{1}$, which is free with $d \log _{1}$ as a basis) with $\mathcal{O}_{\mathcal{X}^{\prime}}$, we get that $g_{1+}^{\#}\left(\mathcal{G}^{\#}\right)[-1]$ is the complex $g_{1 *} \mathcal{G}^{\#} \stackrel{\partial_{1}}{\longrightarrow} g_{1 *} \mathcal{G}^{\#}\left(\right.$ resp. $g_{1+}^{b}\left(\mathcal{G}^{b}\right)[-1]$ is $\left.g_{1 *} \mathcal{G}^{b} \stackrel{t_{1} \partial_{f}}{\longrightarrow} g_{1 *} \mathcal{G}^{b}\right)$.

Remark 3.4.8. Beware that the sequence (3.4.7.4) is not anymore exact if we replace the level 0 by any level $m$ (the proof is a computation involving Koszul complexes, the computation only works at level $0)$. But remark that since the extension $\widehat{\mathcal{D}}_{\mathcal{X}^{\prime} \#, \mathbb{Q}}^{(0)} \rightarrow \widehat{\mathcal{D}}_{\mathcal{X}^{\prime} \#, \mathbb{Q}}^{(m)}$ is flat, since $\widehat{\mathcal{D}}_{\mathcal{Z}_{1}^{\#} \leftarrow \mathcal{X}^{\prime \prime}, \mathbb{Q}}^{(0)} \otimes_{\widehat{\mathcal{D}}_{\mathcal{X}^{\prime} \#, \mathbb{Q}}^{(0)}} \widehat{\mathcal{D}}_{\mathcal{X}^{\prime \prime}, \mathbb{Q}}^{(m)} \stackrel{\sim}{\rightarrow}$ $\widehat{\mathcal{D}}_{\mathcal{Z}_{1}^{\#} \leftarrow \mathcal{X}^{\prime \#}, \mathbb{Q}}^{(m)}:=g_{1 \mathrm{~d}}^{*}\left(\widehat{\mathcal{D}}_{\mathcal{Z}_{1}^{\#}}^{(m)} \otimes_{\mathcal{O}_{\mathcal{Z}_{1}}} \omega_{\mathcal{Z}_{1}^{\#}}^{-1}\right) \otimes_{\mathcal{O}_{\mathcal{X}^{\prime}}} \omega_{\mathcal{X}^{\prime} \#}$, then this is true after applying the functor $-\otimes_{\mathbb{Z}} \mathbb{Q}$.

Lemma 3.4.9. Let $\mathcal{P}$ be a smooth formal scheme, $\mathcal{T}$ be a strict normal crossing divisor on $\mathcal{P}, \mathcal{P} \#:=(\mathcal{P}, \mathcal{T})$, $H$ be a divisor of $P$. Consider the following diagram of coherent $\mathcal{D}_{\mathcal{P} \#, \mathbb{Q}}^{\dagger}$-modules:

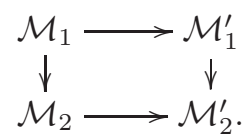

We suppose that the canonical morphism $\mathcal{M}_{2}^{\prime} \rightarrow \mathcal{M}_{2}^{\prime}\left({ }^{\dagger} H\right):=\mathcal{D}_{\mathcal{P} \#}^{\dagger}\left({ }^{\dagger} H\right)_{\mathbb{Q}} \otimes_{\mathcal{D}_{\mathcal{P} \#, \mathbb{Q}}^{\dagger}} \mathcal{M}$ is an isomorphism. Then the diagram is commutative if and only if it is so after restricting to $\mathcal{P} \backslash H$.

Proof. Suppose the diagram commutative after restricting to $\mathcal{P}^{\prime}:=\mathcal{P} \backslash H$. From the diagram (3.4.9.1), we get two morphisms $f, g: \mathcal{M}_{1} \rightarrow \mathcal{M}_{2}^{\prime}$ and we have to check that $f-g=0$. Let $\mathcal{N}^{\prime}$ be the image of $f-g$. Since by hypothesis $\mathcal{N}^{\prime}\left({ }^{\dagger} H\right)\left|\mathcal{P}^{\prime}=\mathcal{N}^{\prime}\right| \mathcal{P}^{\prime}=0$, since $\mathcal{N}^{\prime}\left({ }^{\dagger} H\right)$ is $\mathcal{D}_{\mathcal{P} \#}^{\dagger}\left({ }^{\dagger} H\right)_{\mathbb{Q}^{-c o h e r e n t}}$, from [Car09a, 4.8] we get $\mathcal{N}^{\prime}\left({ }^{\dagger} H\right)=0$. Since the composition $\mathcal{N}^{\prime} \rightarrow \mathcal{N}^{\prime}\left({ }^{\dagger} H\right) \rightarrow \mathcal{M}_{2}^{\prime}\left({ }^{\dagger} H\right) \stackrel{\sim}{\sim} \mathcal{M}_{2}^{\prime}$ is injective, then so is $\mathcal{N}^{\prime} \rightarrow \mathcal{N}^{\prime}\left({ }^{\dagger} H\right)$.

Proposition 3.4.10. (i) The following diagram of holonomic $\mathcal{D}_{\mathcal{X}^{\#}, \mathbb{Q}^{-m o d u l e s}}^{\dagger}$

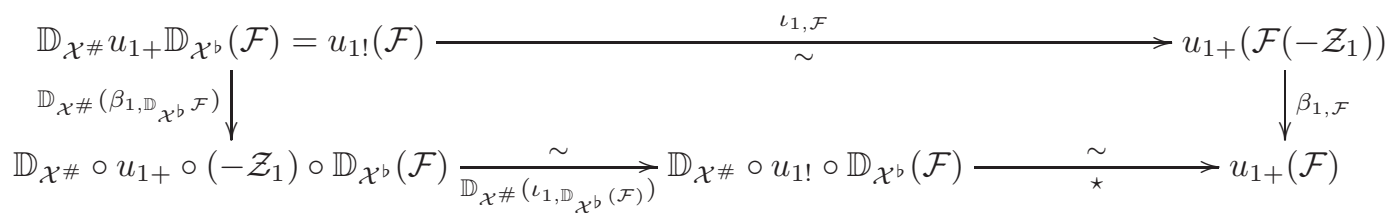

where $\beta_{1, \mathcal{F}}\left(\right.$ resp. $\left.\iota_{1, \mathcal{F}}\right)$ is defined in 3.4 .5 (resp. (3.4.4.2)) and similarly if we replace $\mathcal{F}$ by $\mathbb{D}_{\mathcal{X}^{b}}(\mathcal{F})$, where $\star$ is induced by functoriality using the biduality isomorphisms $\mathbb{D}_{\mathcal{X}^{b}} \circ \mathbb{D}_{\mathcal{X}^{b}} \stackrel{\sim}{\rightarrow} \mathrm{id}$ and $\mathbb{D}_{\mathcal{X}^{\#}} \circ \mathbb{D}_{\mathcal{X}^{\#}} \stackrel{\sim}{\rightarrow} \mathrm{id}$, is commutative. 


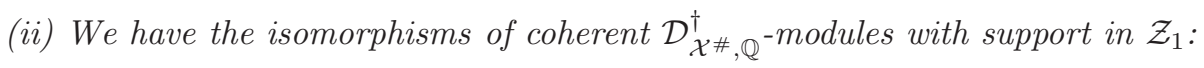

$$
\operatorname{ker}\left(\beta_{1, \mathcal{F}}\right) \stackrel{\sim}{\rightarrow} \mathbb{D}_{\mathcal{X} \#}\left(\operatorname{coker}\left(\beta_{1, \mathbb{D}_{\mathcal{X}^{b}}(\mathcal{F})}\right)\right), \quad \operatorname{coker}\left(\beta_{1, \mathcal{F}}\right) \stackrel{\sim}{\rightarrow} \mathbb{D}_{\mathcal{X} \#}\left(\operatorname{ker}\left(\beta_{1, \mathbb{D}_{\mathcal{X}^{b}}(\mathcal{F})}\right)\right) .
$$

Proof. Let us show (i). By Lemma (3.4.4.5), we have the isomorphism $u_{1+}(\mathcal{F}) \stackrel{\sim}{\rightarrow} \mathcal{F}\left({ }^{\dagger} Z_{1}\right)$. Hence, since the diagram is commutative outside of $Z_{1}$, the commutativity follows by Lemma 3.4.9. The second part of the proposition is straightforward from the first one and (3.4.6.5).

Lemma 3.4.11. With the notation 3.4.5, we have the canonical isomorphisms of $\operatorname{Isoc}^{0}\left(\mathcal{Z}_{1}^{\#} / K\right)$

$$
i_{1}^{\# !}\left(\operatorname{ker}\left(\beta_{1, \mathcal{F}}\right)\right) \stackrel{\sim}{\rightarrow} \operatorname{ker}\left(N_{1, \mathcal{F}}\right), \quad i_{1}^{\# !}\left(\operatorname{coker}\left(\beta_{1, \mathcal{F}}\right)\right) \stackrel{\sim}{\rightarrow} \operatorname{coker}\left(N_{1, \mathcal{F}}\right) .
$$

Proof. 1) First we reduce to the case where $f=$ id. It is straightforward that $\operatorname{ker}\left(N_{1, \mathcal{F}}\right)$ and $\operatorname{coker}\left(N_{1, \mathcal{F}}\right)$ are objects of $\operatorname{Isoc}^{0}\left(\mathcal{Z}_{1}^{\#} / K\right)$. Concerning the left terms of (3.4.11.1), this has been checked in 3.4.6. Now, let us check the isomorphisms. From the relative duality isomorphism satisfied by $i_{1}^{\#}$ (see (3.4.2.1)) and

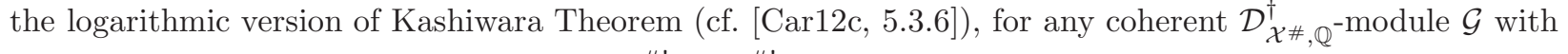
support in $\mathcal{Z}_{1}$, we get the isomorphism $\mathbb{D}_{\mathcal{Z}_{1}^{\#}} i_{1}^{\# !} \mathcal{G} \cong i_{1}^{\# !} \mathbb{D}_{\mathcal{X}^{\#}} \mathcal{G}$. Thus, by duality, using Lemma 3.2.5, (3.4.10.1) and (3.4.1.1), it is sufficient to check the second isomorphism. Since $f$ is log-étale, then $f^{b !}: \operatorname{Isoc}\left(\mathcal{X}^{b} / K\right) \rightarrow$ $\operatorname{Isoc}\left(\mathcal{X}^{\prime b} / K\right)\left(\operatorname{resp} . f^{b !}: \operatorname{Isoc}^{0}\left(\mathcal{X}^{b} / K\right) \rightarrow \operatorname{Isoc}^{0}\left(\mathcal{X}^{\prime b} / K\right)\right)$ is an exact functor. Put $\mathcal{F}^{\prime}:=f^{b !}(\mathcal{F}), \alpha_{1, \mathcal{F}^{\prime}}^{\prime}:=$ $f^{b !}\left(\alpha_{1, \mathcal{F}}\right): \mathcal{F}^{\prime}\left(-\mathcal{Z}_{1}\right) \hookrightarrow \mathcal{F}^{\prime}$ the canonical inclusion, $\beta_{1, \mathcal{F}^{\prime}}^{\prime}:=u_{1+}^{\prime}\left(\alpha_{1, \mathcal{F}^{\prime}}^{\prime}\right): u_{1+}^{\prime}\left(\mathcal{F}^{\prime}\left(-\mathcal{Z}_{1}\right)\right) \rightarrow u_{1+}^{\prime}\left(\mathcal{F}^{\prime}\right)$. From [Car12c, 5.4.6.1], we get $u_{1+}^{\prime} \circ f^{b !} \stackrel{\sim}{\longrightarrow} f^{\# !} \circ u_{1+}$ for coherent complexes. Hence, $f^{\# !}\left(\beta_{1, \mathcal{F}}\right) \stackrel{\sim}{\rightarrow} \beta_{1, \mathcal{F}^{\prime}}^{\prime}$. This yields

$$
\left.i_{1}^{\prime \# !} \operatorname{coker}\left(\beta_{1, \mathcal{F}^{\prime}}^{\prime}\right)\right) \stackrel{\sim}{\longrightarrow} i_{1}^{\prime \# !} f \# ! \operatorname{coker}\left(\beta_{1, \mathcal{F}}\right) \stackrel{\sim}{\longrightarrow} i_{1}^{\# !} \operatorname{coker}\left(\beta_{1, \mathcal{F}}\right)
$$

By definition, we remark $N_{1, \mathcal{F}}=N_{1, \mathcal{F}^{\prime}}$. So, we are reduced to the case where $f=$ id.

2) Suppose $f=$ id. Since $i_{1}^{\#}$ is an exact closed immersion, then from the logarithmic version of BerthelotKashiwara's theorem (cf. [Car12c, 5.3.6]) the functors $i_{1}^{\# !}$ and $i_{1+}^{\#}$ induce quasi-inverse exact functors be-

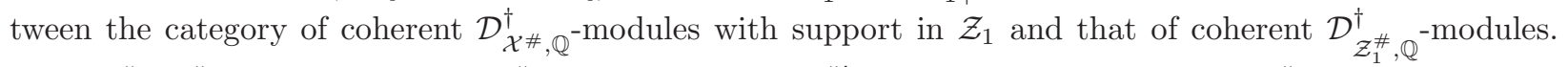
Since $g_{1}^{\#} \circ i_{1+}^{\#}=\mathrm{id}$, this yields $g_{1+}^{\#}\left(\operatorname{coker}\left(\beta_{1, \mathcal{F}}\right)\right) \stackrel{\sim}{\rightarrow} i_{1}^{\# !} \operatorname{coker}\left(\beta_{1, \mathcal{F}}\right)$, which implies $g_{1+}^{\#}\left(\operatorname{coker}\left(\beta_{1, \mathcal{F}}\right)\right) \stackrel{\sim}{\rightarrow}$ $\mathcal{H}^{0} g_{1+}^{\#}\left(\operatorname{coker}\left(\beta_{1, \mathcal{F}}\right)\right)$. From $(3.4 .7 .5)$, the functor $\mathcal{H}^{0} g_{1+}^{\#}$ is right exact and then $\mathcal{H}^{0} g_{1+}^{\#}\left(\operatorname{coker}\left(\beta_{1, \mathcal{F}}\right)\right) \stackrel{\sim}{\rightarrow}$ $\operatorname{coker}\left(\mathcal{H}^{0} g_{1+}^{\#}\left(\beta_{1, \mathcal{F}}\right)\right)$.

From (3.4.4.4), $\mathcal{H}^{i} u_{1+}(\mathcal{G})=0$ for any $i \neq 0$ and $\mathcal{G} \in \operatorname{Isoc}\left(\mathcal{X}^{b} / K\right)$. Since $g_{1}^{\#} \circ u_{1}=g_{1}^{b}$, this implies

$$
\left(\mathcal{H}^{0} g_{1+}^{\#}\right)\left(\beta_{1, \mathcal{F}}\right)=\left(\mathcal{H}^{0} g_{1+}^{\#}\right) \circ u_{1+}\left(\alpha_{1, \mathcal{F}}\right) \stackrel{\sim}{\longrightarrow} \mathcal{H}^{0} g_{1+}^{b}\left(\alpha_{1, \mathcal{F}}\right) .
$$

Since the functor $\mathcal{H}^{0} g_{1+}^{b}$ is right exact (see (3.4.7.6)), we get the second isomorphism: $\operatorname{coker}\left(\mathcal{H}^{0} g_{1+}^{\#}\right)\left(\beta_{1, \mathcal{F}}\right) \stackrel{\sim}{\rightarrow}$ $\operatorname{coker}\left(\mathcal{H}^{0} g_{1+}^{b}\left(\alpha_{1, \mathcal{F}}\right)\right) \stackrel{\sim}{\rightarrow} \mathcal{H}^{0} g_{1+}^{b} \operatorname{coker}\left(\alpha_{1, \mathcal{F}}\right)$ Since coker $\left(\alpha_{1, \mathcal{F}}\right)=\mathcal{F} / \mathcal{F}\left(-\mathcal{Z}_{1}\right)$ and $\mathcal{H}^{0} g_{1+}^{b} \stackrel{\sim}{\rightarrow} g_{1 *} \operatorname{coker}\left(t_{1} \partial_{1} \cdot\right)$ (see the description after (3.4.7.6)), we get

$$
\mathcal{H}^{0} g_{1+}^{b} \operatorname{coker}\left(\alpha_{1, \mathcal{F}}\right) \stackrel{\sim}{\rightarrow} \operatorname{coker}\left(N_{1, \mathcal{F}}\right) .
$$

By composing all the isomorphisms, the lemma follows.

Notation 3.4.12. Moreover, we get a left $\mathcal{D}_{\mathcal{X} \#}^{\dagger}$-module (and then a left $\mathcal{D}_{\mathcal{X}^{b}}^{\dagger}$-module), by posing $\mathcal{O}_{\mathcal{X}}(\mathfrak{D}):=$ $\mathcal{H o m}_{\mathcal{O}_{\mathcal{X}}}\left(\omega_{\mathcal{X}}, \omega_{\mathcal{X} \#}\right)$. As in 3.4.4, for any integer $n \in \mathbb{Z}$, for any $\mathcal{G}^{\#} \in D^{\mathrm{b}}\left(\mathcal{D}_{\mathcal{X}}^{\dagger}, \mathbb{Q}\right), \mathcal{G}^{b} \in D^{\mathrm{b}}\left(\mathcal{D}_{\mathcal{X}^{b}, \mathbb{Q}}^{\dagger}\right)$, we set $\mathcal{O}_{\mathcal{X}}(n \mathfrak{D}):=\mathcal{O}_{\mathcal{X}}(\mathfrak{D})^{\otimes n}\left(\right.$ where $\otimes n$ means that we tensorise $n$ times as $\mathcal{O}_{\mathcal{X}}$-module), $\mathcal{G}^{\#}(n \mathfrak{D}):=\mathcal{G}^{\#} \otimes_{\mathcal{O}_{\mathcal{X}}}$ $\mathcal{O}_{\mathcal{X}}(n \mathfrak{D})$ and $\mathcal{G}^{b}(n \mathfrak{D}):=\mathcal{G}^{b} \otimes_{\mathcal{O}_{\mathcal{X}}} \mathcal{O}_{\mathcal{X}}(n \mathfrak{D})$. Since $\mathcal{O}_{\mathcal{X}}(n \mathfrak{D})$ is a free $\mathcal{O}_{\mathcal{X}}$-module of rank one, then the induced functors $(n \mathfrak{D}): \operatorname{Mod}\left(\mathcal{D}_{\mathcal{X}^{\#}, \mathbb{Q}}^{\dagger}\right) \rightarrow \operatorname{Mod}\left(\mathcal{D}_{\mathcal{X}^{\#}, \mathbb{Q}}^{\dagger}\right)$ and $(n \mathfrak{D}): \operatorname{Mod}\left(\mathcal{D}_{\mathcal{X}^{b}, \mathbb{Q}}^{\dagger}\right) \rightarrow \operatorname{Mod}\left(\mathcal{D}_{\mathcal{X}^{b}, \mathbb{Q}}^{\dagger}\right)$, given respectively by $\mathcal{G}^{\#} \mapsto \mathcal{G}^{\#}(n \mathfrak{D})$ and $\mathcal{G}^{b} \mapsto \mathcal{G}^{b}(n \mathfrak{D})$, are exact. We remark that the functor $\left(\mathcal{Z}_{1}\right): \operatorname{Mod}\left(\mathcal{D}_{\mathcal{X}^{b}, \mathbb{Q}}^{\dagger}\right) \rightarrow \operatorname{Mod}\left(\mathcal{D}_{\mathcal{X}^{b}, \mathbb{Q}}^{\dagger}\right)$ 
defined in 3.4.4 commutes with $(\mathfrak{D})$ and more precisely we have the isomorphism $(\mathfrak{D}) \circ\left(\mathcal{Z}_{1}\right) \stackrel{\sim}{\rightarrow}\left(\mathcal{Z}_{1}\right) \circ(\mathfrak{D}) \stackrel{\sim}{\rightarrow}$ $(\mathcal{Z})$ of functors $\operatorname{Mod}\left(\mathcal{D}_{\mathcal{X}^{b}, \mathbb{Q}}^{\dagger}\right) \rightarrow \operatorname{Mod}\left(\mathcal{D}_{\mathcal{X}^{b}, \mathbb{Q}}^{\dagger}\right)$, where $\mathcal{O}_{\mathcal{X}}(\mathcal{Z}):=\mathcal{H o m}_{\mathcal{O}_{\mathcal{X}}}\left(\omega_{\mathcal{X}}, \omega_{\mathcal{X}^{b}}\right)$ and $(\mathcal{Z}):=\mathcal{O}_{\mathcal{X}}(\mathcal{Z}) \otimes_{\mathcal{O}_{\mathcal{X}}}-$. Similarly, we have the functor $\left(n \mathfrak{D}_{1}\right): \operatorname{Mod}\left(\mathcal{D}_{\mathcal{Z}_{1}^{\#}, \mathbb{Q}}^{\dagger}\right) \rightarrow \operatorname{Mod}\left(\mathcal{D}_{\mathcal{Z}_{1}^{\#}, \mathbb{Q}}^{\dagger}\right)$.

Lemma 3.4.13. With the notation 3.4.12, we have the isomorphism $\left(-\mathfrak{D}_{1}\right) \circ i_{1}^{\# !} \stackrel{\sim}{\rightarrow} i_{1}^{\# !} \circ(-\mathfrak{D})$ of functors $D_{\text {coh }}^{\mathrm{b}}\left(\mathcal{D}_{\mathcal{X} \#, \mathbb{Q}}^{\dagger}\right) \rightarrow D^{\mathrm{b}}\left(\mathcal{D}_{\mathcal{Z}_{1}^{\#}, \mathbb{Q}}^{\dagger}\right)$, where $i_{1}^{\# !}: D_{\mathrm{coh}}^{\mathrm{b}}\left(\mathcal{D}_{\mathcal{X} \#, \mathbb{Q}}^{\dagger}\right) \rightarrow D^{\mathrm{b}}\left(\mathcal{D}_{\mathcal{Z}_{1}^{\#}, \mathbb{Q}}^{\dagger}\right)$ is the extraordinary inverse image (see the definition [Car12c, 5.1.2.1]).

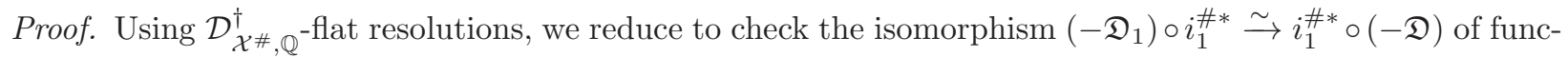
tors $\operatorname{Coh}\left(\mathcal{D}_{\mathcal{X}, \mathbb{Q}}^{\dagger}\right) \rightarrow \operatorname{Mod}\left(\mathcal{D}_{\mathcal{Z}_{1}^{\#, \mathbb{Q}}}^{\dagger}\right)$, where $i_{1}^{\# *}: \operatorname{Coh}\left(\mathcal{D}_{\mathcal{X} \#, \mathbb{Q}}^{\dagger}\right) \rightarrow \operatorname{Mod}\left(\mathcal{D}_{\mathcal{Z}_{1}^{\#}, \mathbb{Q}}^{\dagger}\right)$ is the canonical factorisation induced by the functor $i_{1}^{\# *}$.

Notation 3.4.14. With the notation 3.4 .5 and 3.4 .12 , let $\epsilon_{1, \mathcal{F}}:=u_{+} \circ(-\mathfrak{D})\left(\alpha_{1, \mathcal{F}}\right): u_{+}(\mathcal{F}(-\mathcal{Z})) \rightarrow$ $u_{+}(\mathcal{F}(-\mathfrak{D}))$ and $\theta_{1, \mathcal{E}}: \mathbb{D} \circ \mathbb{D}_{Z}(\mathcal{E}) \rightarrow\left(\dagger Z_{1}\right) \circ \mathbb{D}_{\mathbb{D}^{\prime}} \circ \mathbb{D}_{Z}(\mathcal{E}) \underset{(3.4 .1 .2)}{\stackrel{\sim}{\longrightarrow}} \mathbb{D}_{Z_{1}} \circ \mathbb{D}_{Z}(\mathcal{E})$ be the canonical homomorphisms. Both homomorphisms are isomorphisms outside $Z_{1}$.

Lemma 3.4.15. We keep the notation of 3.4.14.

1. We have the isomorphism

$$
u_{1+} \circ(-\mathfrak{D}) \stackrel{\sim}{\longrightarrow}(-\mathfrak{D}) \circ u_{1+}
$$

of functors $\operatorname{Isoc}\left(\mathcal{X}^{b} / K\right) \rightarrow \operatorname{Coh}\left(\mathcal{D}_{\mathcal{X} \#, \mathbb{Q}}^{\dagger}\right)$.

2. We have the isomorphism

$$
\mathbb{D}_{Z_{1}} \circ \mathbb{D}_{Z} \circ u_{+}(\mathcal{F}) \stackrel{\sim}{\rightarrow} u_{+}(\mathcal{F}(-\mathfrak{D})),
$$

which coincides with (3.4.3.3) outside $Z_{1}$.

Proof. 1) For any $r \geq i>1$, for any section $P$ of $\mathcal{D}_{\mathcal{X} \#, \mathbb{Q}}^{\dagger}$, there exists a section $Q$ of $\mathcal{D}_{\mathcal{X} \#, \mathbb{Q}}^{\dagger}$ such that $t_{i} P=Q t_{i}$ (notice this is not any more true for $t_{1}$ ). Using the description (3.4.4.4) of the functor $u_{1+}$, we get the first assertion.

2) By applying $\left({ }^{\dagger} Z_{1}\right)$ to (3.4.3.3), we obtain the last isomorphism

$$
\mathbb{D}_{Z_{1}} \circ \mathbb{D}_{Z} \circ u_{+}(\mathcal{F}) \underset{(3.4 .1 .2)}{\stackrel{\sim}{\longrightarrow}}\left({ }^{\dagger} Z_{1}\right) \circ \mathbb{D} \circ \mathbb{D}_{Z} \circ u_{+}(\mathcal{F}) \underset{(3.4 .3 .3)}{\stackrel{\sim}{\longrightarrow}}\left({ }^{\dagger} Z_{1}\right)\left(u_{+}(\mathcal{F}(-\mathcal{Z}))\right) .
$$

We have $\left({ }^{\dagger} Z_{1}\right) \circ(-\mathcal{Z})(\mathcal{F}) \stackrel{\sim}{\rightarrow}\left({ }^{\dagger} Z_{1}\right) \circ\left(-\mathcal{Z}_{1}\right) \circ(-\mathfrak{D})(\mathcal{F}) \stackrel{\sim}{\rightarrow}\left({ }^{\dagger} Z_{1}\right) \circ(-\mathfrak{D})(\mathcal{F})$. Since the functor $(-\mathfrak{D})$ commutes canonically with $u_{1+}$ (see (3.4.15.1)) and with $\left({ }^{\dagger} Z_{1}\right)$, by applying $(-\mathfrak{D})$ to the isomorphism $\rho_{1, \mathcal{F}}$ (cf. Lemma (3.4.4.5)), we get $\left({ }^{\dagger} Z_{1}\right) \circ(-\mathfrak{D})(\mathcal{F}) \stackrel{\sim}{\leftarrow} u_{1+} \circ(-\mathfrak{D})(\mathcal{F})$. By composition, we get

$$
\left({ }^{\dagger} Z_{1}\right) \circ(-\mathcal{Z})(\mathcal{F}) \stackrel{\sim}{\rightarrow} u_{1+} \circ(-\mathfrak{D})(\mathcal{F}) .
$$

Applying $v_{+}$to (3.4.15.4), we obtain the second isomorphism:

$$
\left({ }^{\dagger} Z_{1}\right) \circ u_{+} \circ(-\mathcal{Z})(\mathcal{F}) \stackrel{\sim}{\rightarrow} v_{+} \circ\left(^{\dagger} Z_{1}\right) \circ(-\mathcal{Z})(\mathcal{F}) \underset{(3.4 .15 .4)}{\stackrel{\sim}{\rightarrow}} v_{+} \circ u_{1+} \circ(-\mathfrak{D})(\mathcal{F}) \stackrel{\sim}{\rightarrow} u_{+} \circ(-\mathfrak{D})(\mathcal{F})
$$

where the first isomorphism is induced by $\left({ }^{\dagger} Z_{1}\right) \circ u_{+} \stackrel{\sim}{\rightarrow} v_{+} \circ\left({ }^{\dagger} Z_{1}\right)$ from $[\mathrm{CT} 12,2.2 .2]$ and the last one is a consequence of the transitivity of the direct images. This homomorphism (3.4.15.5) is the identity outside $Z_{1}$. By composition of (3.4.15.3) with (3.4.15.5), we get the desired isomorphism, which coincides with (3.4.3.3) outside $Z_{1}$. 
Proposition 3.4.16. (i) With the notation 3.4.14, the following diagram is commutative:

$$
\begin{aligned}
& j !(\mathcal{E})=\mathbb{D} \circ \mathbb{D}_{Z}(\mathcal{E}) \stackrel{\theta_{1, \mathcal{E}}}{\longrightarrow} \mathbb{D}_{Z_{1}} \circ \mathbb{D}_{Z}(\mathcal{E}) \\
& \sim \downarrow(3.4 .15 .2) \\
&(3.4 .3 .3) \downarrow \sim \sim \\
& u_{+}(\mathcal{F}(-\mathcal{Z})) \stackrel{\epsilon_{1, \mathcal{F}}}{\longrightarrow} u_{+}(\mathcal{F}(-\mathfrak{D})) .
\end{aligned}
$$

(ii) We have the canonical isomorphisms:

$$
\mathcal{H}_{Z_{1}}^{\dagger 0}\left(j_{!}(\mathcal{E})\right) \stackrel{\sim}{\rightarrow} \operatorname{ker}\left(\theta_{1, \mathcal{E}}\right) \stackrel{\sim}{\rightarrow} \operatorname{ker}\left(\epsilon_{1, \mathcal{F}}\right), \quad \mathcal{H}_{Z_{1}}^{\dagger 1}\left(j_{!}(\mathcal{E})\right) \stackrel{\sim}{\rightarrow} \operatorname{coker}\left(\theta_{1, \mathcal{E}}\right) \stackrel{\sim}{\rightarrow} \operatorname{coker}\left(\epsilon_{1, \mathcal{F}}\right) .
$$

Proof. Thanks to Lemma 3.4.9, it is sufficient to check the commutativity of the square (3.4.16.1) outside $Z_{1}$. Since the horizontal morphisms of the square (3.4.16.1) are the identity outside $Z_{1}$, this commutativity is a consequence of the second statement of 3.4.15. Let us check the part (ii) of the proposition. Since $\left({ }^{\dagger} Z_{1}\right) \circ j_{!}(\mathcal{E}) \stackrel{\sim}{\rightarrow} \mathbb{D}_{Z_{1}} \circ \mathbb{D}_{Z}(\mathcal{E})$, the exact triangle of localisation of $j_{!}(\mathcal{E})$ with respect to the divisor $Z_{1}$ can be written in the form of

$$
\mathbb{R} \underline{\Gamma}_{Z_{1}}^{\dagger} j_{!}(\mathcal{E}) \rightarrow j_{!}(\mathcal{E}) \underset{\theta_{1, \mathcal{E}}}{\longrightarrow} \mathbb{D}_{Z_{1}} \circ \mathbb{D}_{Z}(\mathcal{E}) \rightarrow \mathbb{R} \underline{\Gamma}_{Z_{1}}^{\dagger} j_{!}(\mathcal{E})[1] .
$$

From (3.4.16.3), we obtain both first isomorphisms. We get both second isomorphisms from the part (i) of the proposition.

Proposition 3.4.17. With the notation 3.4.14, we have the canonical isomorphisms:

$$
i_{1}^{!} \operatorname{ker}\left(\epsilon_{1, \mathcal{F}}\right) \stackrel{\sim}{\rightarrow} w_{1+}\left(\operatorname{ker}\left(N_{1, \mathcal{F}}\right)\left(-\mathfrak{D}_{1}\right)\right), \quad i_{1}^{!} \operatorname{coker}\left(\epsilon_{1, \mathcal{F}}\right) \stackrel{\sim}{\rightarrow} w_{1+}\left(\operatorname{coker}\left(N_{1, \mathcal{F}}\right)\left(-\mathfrak{D}_{1}\right)\right) .
$$

Proof. Using (3.4.15.1), with the notation 3.4.5, we get $u_{1+} \circ(-\mathfrak{D})\left(\alpha_{1, \mathcal{F}}\right) \stackrel{\sim}{\rightarrow}(-\mathfrak{D})\left(\beta_{1, \mathcal{F}}\right)$. Thus, since the functor $(-\mathfrak{D}): \operatorname{Coh}\left(\mathcal{D}_{\mathcal{X} \#, \mathbb{Q}}^{\dagger}\right) \rightarrow \operatorname{Coh}\left(\mathcal{D}_{\mathcal{X} \#, \mathbb{Q}}^{\dagger}\right)$ is exact, we get the exact sequence

$$
0 \rightarrow \operatorname{ker}\left(\beta_{1, \mathcal{F}}\right)(-\mathfrak{D}) \rightarrow u_{1+}(\mathcal{F}(-\mathcal{Z})) \stackrel{u_{1+}(-\mathfrak{D})\left(\alpha_{1, \mathcal{F}}\right)}{\longrightarrow} u_{1+}(\mathcal{F}(-\mathfrak{D})) \rightarrow \operatorname{coker}\left(\beta_{1, \mathcal{F}}\right)(-\mathfrak{D}) \rightarrow 0 .
$$

Via the logarithmic version of Berthelot-Kashiwara's theorem [Car12c, 5.3.6]), we get the first isomorphism

$$
\operatorname{ker}\left(\beta_{1, \mathcal{F}}\right)(-\mathfrak{D}) \stackrel{\sim}{\leftarrow} i_{1+}^{\#} \circ i_{1}^{\# !}\left(\operatorname{ker}\left(\beta_{1, \mathcal{F}}\right)(-\mathfrak{D})\right) \underset{3.4 .13}{\stackrel{\sim}{\longrightarrow}} i_{1+}^{\#}\left(\left(-\mathfrak{D}_{1}\right) \circ i_{1}^{\# !}\left(\operatorname{ker}\left(\beta_{1, \mathcal{F}}\right)\right)\right) .
$$

From Lemma 3.4.11 and with the stability of $\operatorname{Isoc}\left(\mathcal{Z}_{1}^{\#} / K\right)$ under the $\left(-\mathfrak{D}_{1}\right)$, we get $\left(-\mathfrak{D}_{1}\right) \circ i_{1}^{\# !}\left(\operatorname{ker}\left(\beta_{1, \mathcal{F}}\right)\right) \in$ $\operatorname{Isoc}\left(\mathcal{Z}_{1}^{\#} / K\right)$. From [Car09a, 5.24], the functor $\mathcal{H}^{0} w_{1+}$ is acyclic over $\operatorname{Isoc}\left(\mathcal{Z}_{1}^{\#} / K\right)$ (i.e. $\mathcal{H}^{i} w_{1+}(\mathcal{G})=0$ for any $\left.\mathcal{G} \in \operatorname{Isoc}\left(\mathcal{Z}_{1}^{\#} / K\right)\right)$. Since the functor $i_{1+}^{\#}\left(\operatorname{resp} . i_{1+}\right)$ is exact over $\operatorname{Isoc}\left(\mathcal{Z}_{1}^{\#} / K\right)\left(\operatorname{resp} . \operatorname{Coh}\left(\mathcal{D}_{\mathcal{Z}_{1}, \mathbb{Q}}^{\dagger}\right)\right)$, since we have by transitivity of direct images $v_{+} \circ i_{1+}^{\#} \stackrel{\sim}{\rightarrow} i_{1+} \circ w_{1+}$, with isomorphism $(\star \star)$ we obtain that $\operatorname{ker}\left(\beta_{1, \mathcal{F}}\right)(-\mathfrak{D})$ is acyclic for $\mathcal{H}^{0} v_{+}$. In the same way, $\operatorname{coker}\left(\beta_{1, \mathcal{F}}\right)(-\mathfrak{D})$ is acyclic for $\mathcal{H}^{0} v_{+}$. Since $\mathcal{F}(-\mathcal{Z})$ and $\mathcal{F}(-\mathcal{D})$ belong to $\operatorname{Isoc}\left(\mathcal{X}^{\mathrm{b}} / K\right)$, then from (3.4.4.4) (resp. [Car09a, 5.24]), they are $\mathcal{H}^{0} u_{1+}$ acyclic (resp. $\mathcal{H}^{0} u_{+}$acyclic). Since $v_{+} \circ u_{1+} \stackrel{\sim}{\rightarrow} u_{+}$, then this implies that $u_{1+}(\mathcal{F}(-\mathcal{Z}))$ and $u_{1+}(\mathcal{F}(-\mathcal{D}))$ are $\mathcal{H}^{0} v_{+}$acyclic. Thus, by applying the functor $\mathcal{H}^{0} v_{+}$to $(\star)$, we obtain the exact sequence:

$$
0 \rightarrow v_{+}\left(\operatorname{ker}\left(\beta_{1, \mathcal{F}}\right)(-\mathfrak{D})\right) \rightarrow u_{+}(\mathcal{F}(-\mathcal{Z})) \stackrel{\epsilon_{1, \mathcal{F}}}{\longrightarrow} u_{+}(\mathcal{F}(-\mathfrak{D})) \rightarrow v_{+}\left(\operatorname{coker}\left(\beta_{1, \mathcal{F}}\right)(-\mathfrak{D})\right) \rightarrow 0 .
$$

Using $v_{+} \circ i_{1+}^{\#} \stackrel{\sim}{\rightarrow} i_{1+} \circ w_{1+}$, we get the first isomorphism:

$$
v_{+}\left(\operatorname{ker}\left(\beta_{1, \mathcal{F}}\right)(-\mathfrak{D})\right) \underset{v_{+}(\star \star)}{\sim} i_{1+} \circ w_{1+} \circ\left(-\mathfrak{D}_{1}\right) \circ i_{1}^{\# !}\left(\operatorname{ker}\left(\beta_{1, \mathcal{F}}\right)\right) \underset{(3.4 .11 .1)}{\stackrel{\sim}{\longrightarrow}} i_{1+} \circ w_{1+} \circ\left(-\mathfrak{D}_{1}\right)\left(\operatorname{ker}\left(N_{1, \mathcal{F}}\right)\right) .
$$

Considering (3.4.17.2) and (3.4.17.3), we get $\operatorname{ker}\left(\epsilon_{1, \mathcal{F}}\right) \stackrel{\sim}{\rightarrow} i_{1+} w_{1+}\left(\operatorname{ker}\left(N_{1, \mathcal{F}}\right)\left(-\mathfrak{D}_{1}\right)\right)$. Hence, using BerthelotKashiwara theorem, we get the first isomorphism. By replacing "ker" by "coker" in the proof above, we get the second isomorphism, and we conclude the proof. 
Remark 3.4.18. With the notation 3.4.5 and 3.4.14, we remark that the homomorphisms $\epsilon_{1, \mathcal{F}}$ and $\beta_{1, \mathcal{F}}$ are equal outside of $D$, and the isomorphisms in Lemma 3.4.11 and Lemma 3.4.17 are the same outside of $D_{1}$. In particular, when $f=$ id and $\mathcal{D}$ is empty (i.e. $r=1$ ), then the isomorphism of Lemma 3.4.17 is simply the one induced by composition from (3.4.11.3) and (3.4.11.4).

Theorem 3.4.19. We put $\mathcal{Y}_{1}:=\mathcal{Z}_{1} \backslash \mathfrak{D}_{1}$, and let $j_{1}:=(\star$, id, id $):\left(Y_{1}, Z_{1}, \mathcal{Z}_{1}\right) \rightarrow\left(Z_{1}, Z_{1}, \mathcal{Z}_{1}\right)$ be the canonical

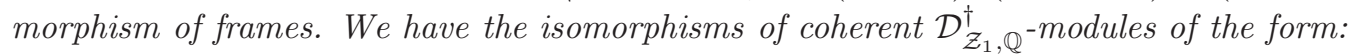

$$
i_{1}^{!} \mathcal{H}_{Z_{1}}^{\dagger 1}\left(j_{!}(\mathcal{E})\right) \stackrel{\sim}{\rightarrow} j_{1 !} \circ\left({ }^{\dagger} D_{1}\right)\left(\operatorname{coker} N_{1, \mathcal{F}}\right), \quad i_{1}^{!} \mathcal{H}_{Z_{1}}^{\dagger 0}\left(j_{!}(\mathcal{E})\right) \stackrel{\sim}{\rightarrow} j_{1 !} \circ\left({ }^{\dagger} D_{1}\right)\left(\operatorname{ker} N_{1, \mathcal{F}}\right)
$$

Proof. The composition of $i_{1}^{!}(3.4 .16 .2)$ with (3.4.17.1) is of the form

$$
i_{1}^{!} \mathcal{H}_{Z_{1}}^{\dagger 1}\left(j_{!}(\mathcal{E})\right) \stackrel{\sim}{\rightarrow} w_{1+}\left(\operatorname{coker} N_{1, \mathcal{F}}\left(-\mathfrak{D}_{1}\right)\right) .
$$

Since coker $N_{1, \mathcal{F}} \in \operatorname{Isoc}^{0}\left(\mathcal{Z}_{1}^{\#} / K\right)$, as (3.4.3.3) and (3.4.4.5), we get

$$
w_{1+}\left(\operatorname{coker} N_{1, \mathcal{F}}\left(-\mathfrak{D}_{1}\right)\right) \underset{(3.4 .3 .3)}{\stackrel{\sim}{\longrightarrow}} j_{1 !} \circ w_{1+}\left(\operatorname{coker} N_{1, \mathcal{F}}\right) \underset{(3.4 .4 .5)}{\stackrel{\sim}{\longrightarrow}} j_{1 !} \circ\left({ }^{\dagger} D_{1}\right)\left(\operatorname{coker} N_{1, \mathcal{F}}\right) .
$$

By composition, we obtain the left isomorphism of (3.4.19.1). We get similarly the right isomorphism of (3.4.19.1) replacing everywhere cokernels by kernels.

\subsection{An isomorphism compatible with Frobenius}

The goal of this subsection is Theorem 3.5.13 which show the compatibility with Frobenius of the main isomorphisms (3.4.19.1) of the previous subsection.

3.5.1. In this subsection (except for Theorem 3.5.13), we keep the following notation and hypotheses : Let $\phi: \mathcal{X} \rightarrow \widehat{\mathbb{A}}_{\mathcal{V}}^{d}$ be an étale morphism of affine formal $\mathcal{V}$-schemes and let $t_{1}, \ldots, t_{d}$ be the corresponding local coordinates and $\partial_{1}, \ldots, \partial_{d}$ be the corresponding derivations. We set $A:=\Gamma\left(\mathcal{X}, \mathcal{O}_{\mathcal{X}}\right), B:=A / t_{1} A$ and $\mathcal{Z}_{1}:=\operatorname{Spf} B$. We suppose that there exists a smooth morphism $g_{1}: \mathcal{X} \rightarrow \mathcal{Z}_{1}$ such that $g_{1} \circ i_{1}=$ id and the $\mathcal{Z}_{1}$-morphism of the form $h: \mathcal{X} \rightarrow \widehat{\mathbb{A}}_{\mathcal{Z}_{1}}^{1}$ induced by $t_{1}$ is étale. Let $\bar{t}_{2}, \ldots, \bar{t}_{d}$ be the global sections of $\mathcal{O}_{\mathcal{Z}_{1}}$ induced by $t_{2}, \ldots, t_{d}$ and let $\phi_{1}: \mathcal{Z}_{1} \rightarrow \widehat{\mathbb{A}}_{\mathcal{V}}^{d-1}$ be the étale morphism induced by the local coordinates $\bar{t}_{2}, \ldots, \bar{t}_{d}$. We keep the notation the canonical absolute Frobenius $F_{\widehat{\mathbb{A}}_{\mathcal{V}}^{d}}, F_{\widehat{\mathbb{A}}_{\mathcal{V}}^{d-1}}, F_{\mathcal{X}}, F_{\mathcal{Z}_{1}}$ of the paragraph 3.1.3. We set $F_{\mathcal{X} / \mathcal{Z}_{1}}=\left(F_{\mathcal{X}}, g_{1}\right): \mathcal{X} \rightarrow \mathcal{X}^{\prime}:=\mathcal{X} \times \mathcal{Z}_{1}, F_{\mathcal{Z}_{1}} \mathcal{Z}_{1}$. By abuse of notation, we also simply denote by $F_{\mathcal{Z}_{1}}$ the morphisms id $\times F_{\mathcal{Z}_{1}}: \mathcal{X}^{\prime} \rightarrow \mathcal{X}$ and id $\times F_{\mathcal{Z}_{1}}: \widehat{\mathbb{A}}_{\mathcal{Z}_{1}}^{1} \rightarrow \widehat{\mathbb{A}}_{\mathcal{Z}_{1}}^{1}$ induced by $F_{\mathcal{Z}_{1}}$. Similarly, we denote by $\phi_{1}$ the morphism id $\times \phi_{1}: \widehat{\mathbb{A}}_{\mathcal{Z}_{1}}^{1} \rightarrow \widehat{\mathbb{A}}_{\mathcal{V}}^{d}$. Hence, to sum-up, we get the commutative diagrams:
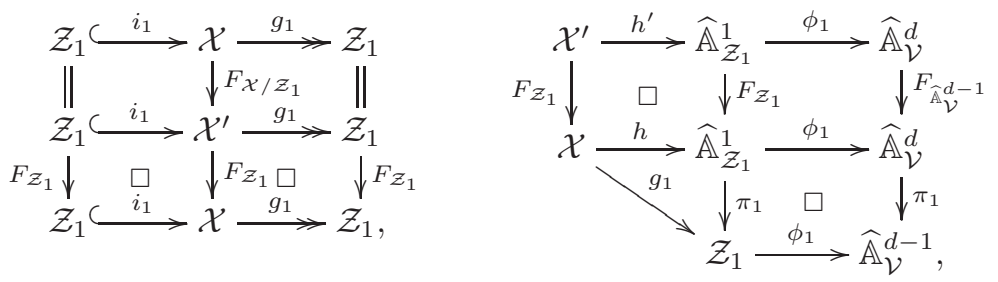

where the symbol $\square$ means the cartesianity of the corresponding square, where $\pi_{1}: \widehat{\mathbb{A}}_{\mathcal{V}}^{d} \rightarrow \widehat{\mathbb{A}}_{\mathcal{V}}^{d-1}$ is the projection on the last $d-1$ coordinates and $h^{\prime}: \mathcal{X}^{\prime} \rightarrow \widehat{\mathbb{A}}_{\mathcal{Z}_{1}}^{1}$ is the canonical projection (via the isomorphism $\left.\mathcal{X}^{\prime} \stackrel{\sim}{\rightarrow} \mathcal{X} \times_{h, \widehat{\mathbb{A}}_{\mathcal{Z}_{1}}^{1}, F_{\mathcal{Z}_{1}}} \widehat{\mathbb{A}}_{\mathcal{Z}_{1}}^{1}\right)$. We denote by $t_{1}^{\prime}, \ldots, t_{d}^{\prime}$ the local coordinate of $\mathcal{X}^{\prime}$ corresponding to the étale map $\phi_{1} \circ h^{\prime}$ and by $\partial_{1}^{\prime}, \ldots, \partial_{d}^{\prime}$ the corresponding derivations. Hence, we compute that the image of $t_{1}$ (resp. $\left.t_{2}, \ldots, t_{d}\right)$ under $F_{\mathcal{Z}_{1}}^{*}: \Gamma\left(\mathcal{X}, \mathcal{O}_{\mathcal{X}}\right) \rightarrow \Gamma\left(\mathcal{X}^{\prime}, \mathcal{O}_{\mathcal{X}^{\prime}}\right)$ is $t_{1}^{\prime}\left(\right.$ resp. $\left.t_{2}^{\prime q}, \ldots, t_{d}^{\prime q}\right)$. On the other hand, the image of $t_{1}^{\prime}\left(\right.$ resp. $\left.t_{2}^{\prime}, \ldots, t_{d}^{\prime}\right)$ under $F_{\mathcal{X} / \mathcal{Z}_{1}}^{*}: \Gamma\left(\mathcal{X}^{\prime}, \mathcal{O}_{\mathcal{X}^{\prime}}\right) \rightarrow \Gamma\left(\mathcal{X}, \mathcal{O}_{\mathcal{X}}\right)$ is $t_{1}^{q}\left(\right.$ resp. $\left.t_{2}, \ldots, t_{d}\right)$. Finally, we remark that, 
via $h\left(\right.$ resp. $\left.h^{\prime}\right), t_{1}$ (resp. $t_{1}^{\prime}$ ) is a relative local coordinate of $\mathcal{X} / \mathcal{Z}_{1}$ (resp. $\mathcal{X}^{\prime} / \mathcal{Z}_{1}$ ). In particular, $\Omega_{\mathcal{X} / \mathcal{Z}_{1}}^{1}$ is

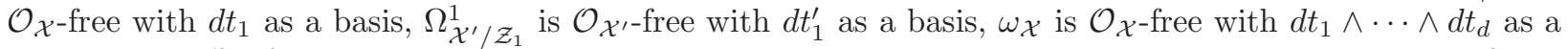
basis, $\omega_{\mathcal{X}^{b}}$ is $\mathcal{O}_{\mathcal{X}}$-free with $d \log _{1} \wedge d t_{2} \wedge \cdots \wedge d t_{d}$ as a basis. Hence, since the local coordinates are fixed, to make some computations, we will sometimes identify $\Omega_{\mathcal{X} / \mathcal{Z}_{1}}^{1}$, (resp. $\omega_{\mathcal{X}}$, resp. $\left.\omega_{\mathcal{X}^{b}}\right)$ with $\mathcal{O}_{\mathcal{X}}$ and $\Omega_{\mathcal{X}^{\prime}}^{1} / \mathcal{Z}_{1}$ with $\mathcal{O}_{\mathcal{X}^{\prime}}$.

3.5.2. As in 3.1.6, let $\mathcal{X}^{\mathrm{b}}:=\left(\mathcal{X}, \mathcal{Z}_{1}\right), u: \mathcal{X}^{b} \rightarrow \mathcal{X},\left(\mathcal{F}, \phi_{\mathcal{F}}\right) \in F-\operatorname{Isoc}\left(\mathcal{X}^{b} / K\right),\left(\mathcal{E}, \phi_{\mathcal{E}}\right):=u_{+}\left(\mathcal{F}, \phi_{\mathcal{F}}\right)$. We put $\mathcal{E}^{\prime}:=F_{\mathcal{Z}_{1}}^{*}(\mathcal{E}), \mathcal{F}^{\prime}:=F_{\mathcal{Z}_{1}}^{*}(\mathcal{F})$. We get the commutative diagram:

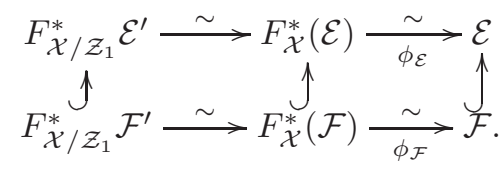

3.5.3 (Relative de Rham complex and Frobenius). Since de Rham complexes commute with base changes, for any $\mathcal{G} \in D_{\text {coh }}^{\mathrm{b}}\left(\mathcal{D}_{\mathcal{X}, \mathbb{Q}}^{\dagger}\right)$, we have $F_{\mathcal{Z}_{1}}^{*} g_{1 *}\left(\Omega_{\mathcal{X} / \mathcal{Z}_{1}}^{\bullet} \otimes_{\mathcal{O}_{\mathcal{X}}} \mathcal{G}\right) \stackrel{\sim}{\rightarrow} g_{1 *} F_{\mathcal{Z}_{1}}^{*}\left(\Omega_{\mathcal{X} / \mathcal{Z}_{1}}^{\bullet} \otimes_{\mathcal{O}_{\mathcal{X}}} \mathcal{G}\right) \stackrel{\sim}{\rightarrow} g_{1 *}\left(\Omega_{\mathcal{X}^{\prime} / \mathcal{Z}_{1}}^{\bullet_{\mathcal{O}_{\mathcal{X}}}} F_{\mathcal{Z}_{1}}^{*} \mathcal{G}\right)$ (recall that $\mathcal{X}$ is affine and then $g_{1 *}$ is exact). Moreover, as in [Ber00, 4.3.5], we check the canonical morphism induced by functoriality under $F_{\mathcal{X} / \mathcal{Z}_{1}}: \mathcal{X} \rightarrow \mathcal{X}^{\prime}$ between de Rham complexes

$$
\Omega_{\mathcal{X}^{\prime} / \mathcal{Z}_{1}}^{\bullet} \otimes_{\mathcal{O}_{\mathcal{X}^{\prime}}} \mathcal{G}^{\prime} \rightarrow \Omega_{\mathcal{X} / \mathcal{Z}_{1}}^{\bullet} \otimes_{\mathcal{O}_{\mathcal{X}}} F_{\mathcal{X} / \mathcal{Z}_{1}}^{*} \mathcal{G}^{\prime}
$$

is a quasi-isomorphism for any $\mathcal{G}^{\prime} \in D_{\text {coh }}^{\mathrm{b}}\left(\mathcal{D}_{\mathcal{X}^{\prime}, \mathbb{Q}}^{\dagger}\right)$. Hence, we get the isomorphism

$$
F_{\mathcal{Z}_{1}}^{*} g_{1 *}\left(\Omega_{\mathcal{X} / \mathcal{Z}_{1}}^{\bullet} \otimes_{\mathcal{O}_{\mathcal{X}}} \mathcal{E}\right) \stackrel{\sim}{\rightarrow} g_{1 *}\left(\Omega_{\mathcal{X}^{\prime} / \mathcal{Z}_{1}} \otimes_{\mathcal{O}_{\mathcal{X}^{\prime}}} \mathcal{E}^{\prime}\right) \stackrel{\sim}{\rightarrow} g_{1 *}\left(\Omega_{\mathcal{X} / \mathcal{Z}_{1}}^{\bullet} \otimes_{\mathcal{O}_{\mathcal{X}}} F_{\mathcal{X}}^{*} \mathcal{E}\right) \underset{\phi_{\mathcal{E}}}{\stackrel{\sim}{\rightarrow}} g_{1 *}\left(\Omega_{\mathcal{X} / \mathcal{Z}_{1}}^{\bullet} \otimes_{\mathcal{O}_{\mathcal{X}}} \mathcal{E}\right)
$$

whose composition is the Frobenius structure of $g_{1 *}\left(\Omega_{\mathcal{X} / \mathcal{Z}_{1}}^{\bullet} \otimes_{\mathcal{O}_{\mathcal{X}}} \mathcal{E}\right)$ induced by that of $\mathcal{E}$.

3.5.4. From [Abe13, 3.15], we have the canonical isomorphism compatible with Frobenius:

$$
g_{1+}(\mathcal{E}) \stackrel{\sim}{\rightarrow} g_{1 *}\left(\Omega_{\mathcal{X} / \mathcal{Z}_{1}}^{\bullet} \otimes_{\mathcal{O}_{\mathcal{X}}} \mathcal{E}\right)[1](1),
$$

where Frobenius structure of the right term is detailed in (3.5.3.2). By identifying $\Omega_{\mathcal{X} / \mathcal{Z}_{1}}^{1}$ (via the basis $d t_{1}$ as $\mathcal{O}_{\mathcal{X}}$-module) with $\mathcal{O}_{\mathcal{X}}$ and applying the functor $\mathcal{H}^{0}$ to the right term of (3.5.4.1), we get a Frobenius structure on coker $\partial_{1}(\mathcal{E})$ (since $\mathcal{X}$ is affine, by abuse of notation we sometimes forget to write $g_{1 *}$ ) from that of $g_{1 *}\left(\Omega_{\mathcal{X} / \mathcal{Z}_{1}} \otimes_{\mathcal{O}_{\mathcal{X}}} \mathcal{E}\right)[1]$. Hence, applying the functor $\mathcal{H}^{0}$ to (3.5.4.1), we get the isomorphism compatible with Frobenius

$$
\mathcal{H}^{0} g_{1+}(\mathcal{E}) \stackrel{\sim}{\rightarrow} \operatorname{coker}_{\partial_{1}}(\mathcal{E})(1) .
$$

Let us describe the Frobenius structure on $\operatorname{coker}_{\partial_{1}}(\mathcal{E})$. Since $F_{\mathcal{X} / \mathcal{Z}_{1 *}}=\mathrm{id}$, we get the adjunction morphism $\mathcal{E}^{\prime} \rightarrow F_{\mathcal{X} / \mathcal{Z}_{1}}^{*} \mathcal{E}^{\prime}$. With the isomorphism $F_{\mathcal{X} / \mathcal{Z}_{1}}^{*} \mathcal{E}^{\prime} \stackrel{\sim}{\rightarrow} F_{\mathcal{X}}^{*} \mathcal{E}$ of (3.5.2.1), this yields adj: $\mathcal{E}^{\prime} \rightarrow F_{\mathcal{X}}^{*} \mathcal{E}$. By identifying $\Omega_{\mathcal{X}^{\prime} / \mathcal{Z}_{1}}^{1}$ with $\mathcal{O}_{\mathcal{X}^{\prime}}$ and $\Omega_{\mathcal{X} / \mathcal{Z}_{1}}^{1}$ with $\mathcal{O}_{\mathcal{X}}$, the canonical quasi-isomorphism $\Omega_{\mathcal{X}^{\prime} / \mathcal{Z}_{1}}^{\boldsymbol{E}_{1}} \otimes_{\mathcal{O}_{\mathcal{X}^{\prime}}} \mathcal{E}^{\prime} \rightarrow$ $\Omega_{\mathcal{X} / \mathcal{Z}_{1}} \otimes_{\mathcal{O}_{\mathcal{X}}} F_{\mathcal{X}}^{*}(\mathcal{E})($ see $(3.5 .3 .1))$ is given by the commutative diagram

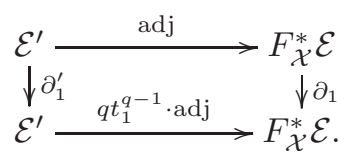

The maps $q t_{1}^{q-1} \cdot$ adj induces the isomorphism coker $_{\partial_{1}^{\prime}}\left(\mathcal{E}^{\prime}\right) \stackrel{\sim}{\rightarrow} \operatorname{coker}_{\partial_{1}}\left(F_{\mathcal{X}}^{*} \mathcal{E}\right)$ given by $\left[x^{\prime}\right] \mapsto\left[q t_{1}^{q-1} \otimes x^{\prime}\right]$. Hence we get

$$
F_{\mathcal{Z}_{1}}^{*} \operatorname{coker}_{\partial_{1}}(\mathcal{E}) \stackrel{\sim}{\longrightarrow} \operatorname{coker}_{\partial_{1}^{\prime}}\left(\mathcal{E}^{\prime}\right) \stackrel{\sim}{\longrightarrow} \operatorname{coker}_{\partial_{1}}\left(F_{\mathcal{X}}^{*} \mathcal{E}\right) \underset{\phi_{\mathcal{E}}}{\sim} \operatorname{coker}_{\partial_{1}}(\mathcal{E}),
$$

where the first homomorphism is an isomorphism because $F_{\mathcal{Z}_{1}}$ is flat. We notice that the composition (3.5.4.4) is indeed by construction the Frobenius structure of coker $\partial_{1}(\mathcal{E})$ which comes from that of $g_{1 *}\left(\Omega_{\mathcal{X} / \mathcal{Z}_{1}} \otimes_{\mathcal{O}_{\mathcal{X}}} \mathcal{E}\right)$ given in (3.5.3.2). 
3.5.5. As in (3.4.7.6), we have the canonical isomorphism (without Frobenius structure):

$$
g_{1+}^{b}(\mathcal{F}) \stackrel{\sim}{\rightarrow} g_{1 *}\left(\Omega_{\mathcal{X}^{b} / \mathcal{Z}_{1}}^{\bullet} \otimes_{\mathcal{O}_{\mathcal{X}}} \mathcal{F}\right)[1] .
$$

By identifying $\Omega_{\mathcal{X}^{b} / \mathcal{Z}_{1}}$ with $\mathcal{O}_{\mathcal{X}}$ and by applying the functor $\mathcal{H}^{0}$, we get the isomorphism

$$
\mathcal{H}^{0} g_{1+}^{b}(\mathcal{F}) \stackrel{\sim}{\rightarrow} \operatorname{coker}_{t_{1} \partial_{1}}(\mathcal{F}) .
$$

Now, let us define a Frobenius structure on $\operatorname{coker}_{t_{1} \partial_{1}}(\mathcal{F})$ as follows (which will be a log analogue of that on $\operatorname{coker}_{\partial_{1}}(\mathcal{E})$ given in (3.5.4.4)) : we denote by adj: $\mathcal{F}^{\prime} \rightarrow F_{\mathcal{X} / \mathcal{Z}_{1}}^{*} \mathcal{F}^{\prime} \stackrel{\sim}{\rightarrow} F_{\mathcal{X}}^{*} \mathcal{F}$ the canonical homomorphism. By (3.2.11.1), we can check that the diagram

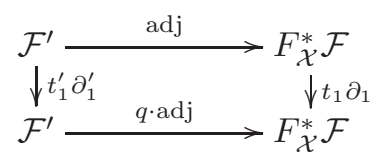

is commutative. Thus the map $q \cdot \operatorname{adj}$ induces the canonical map coker ${ }_{t_{1}^{\prime} \partial_{1}^{\prime}}\left(\mathcal{F}^{\prime}\right) \rightarrow \operatorname{coker}_{t_{1} \partial_{1}}\left(F_{\mathcal{X}}^{*} \mathcal{F}\right)$. We get the Frobenius structure on coker $t_{1} \partial_{1}(\mathcal{F})$ via the homomorphisms:

$$
F_{\mathcal{Z}_{1}}^{*} \operatorname{coker}_{t_{1} \partial_{1}}(\mathcal{F}) \stackrel{\sim}{\rightarrow} \operatorname{coker}_{t_{1}^{\prime} \partial_{1}^{\prime}}\left(\mathcal{F}^{\prime}\right) \underset{q \cdot \text { adj }}{\sim} \operatorname{coker}_{t_{1} \partial_{1}}\left(F_{\mathcal{X}}^{*} \mathcal{F}\right) \underset{\operatorname{coker}_{t_{1} \partial_{1}}(\phi)}{\sim} \operatorname{coker}_{t_{1} \partial_{1}}(\mathcal{F}) .
$$

3.5.6. We have the isomorphisms

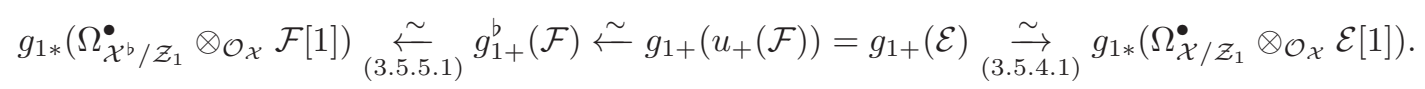

Lemma 3.5.7. By applying $\mathcal{H}^{0}$ to the isomorphism (3.5.6.1), we obtain the isomorphism coker $_{t_{1} \partial_{1}}(\mathcal{F}) \stackrel{\sim}{\rightarrow}$ coker $\partial_{1}(\mathcal{E})$. This isomorphism commutes with Frobenius structures defined respectively in (3.5.5.4) and (3.5.4.4).

Proof. 1) Let us recall the definition of the isomorphisms appearing in (3.5.6.1).

a) The isomorphism $g_{1+}^{b}(\mathcal{F}) \stackrel{\sim}{\leftarrow} g_{1+} \circ u_{+}$is by definition (notice that here $u^{-1}=$ id and $u_{*}=$ id and then we do not need to use a projection formula) :

$$
g_{1+}^{b}(\mathcal{F})=g_{1 *}\left(\mathcal{D}_{\mathcal{Z}_{1} \leftarrow \mathcal{X}^{b}}^{\dagger} \otimes_{\mathcal{D}_{\mathcal{X}^{b}}^{\dagger}}^{\mathbb{L}} \mathcal{F}\right) \stackrel{\sim}{\rightarrow} g_{1 *}\left(\mathcal{D}_{\mathcal{Z}_{1} \leftarrow \mathcal{X}}^{\dagger} \otimes_{\mathcal{D}_{\mathcal{X}}^{\dagger}}^{\mathbb{L}}\left(\mathcal{D}_{\mathcal{X} \leftarrow \mathcal{X}^{b}}^{\dagger} \otimes_{\mathcal{D}_{\mathcal{X}^{b}}^{\dagger}}^{\mathbb{L}} \mathcal{F}\right)\right) \stackrel{\sim}{\rightarrow} g_{1+}\left(u_{+}(\mathcal{F})\right),
$$

where the first isomorphism comes from the canonical isomorphism

$$
\mathcal{D}_{\mathcal{Z}_{1} \leftarrow \mathcal{X}}^{\dagger} \otimes_{\mathcal{D}_{\mathcal{X}}^{\dagger}}^{\mathbb{L}} \mathcal{D}_{\mathcal{X} \leftarrow \mathcal{X}^{b}}^{\dagger} \stackrel{\sim}{\rightarrow} \mathcal{D}_{\mathcal{Z}_{1} \leftarrow \mathcal{X}^{b}}^{\dagger}
$$

b) From (3.4.7.4), we get the exact sequence

$$
0 \rightarrow \mathcal{D}_{\mathcal{X}^{(b)}, \mathbb{Q}}^{\dagger} \rightarrow \Omega_{\mathcal{X}^{(b)} / \mathcal{Z}_{1}}^{1} \otimes_{\mathcal{O}_{\mathcal{X}}} \mathcal{D}_{\mathcal{X}^{(b)}, \mathbb{Q}}^{\dagger} \rightarrow \mathcal{D}_{\mathcal{Z}_{1} \leftarrow \mathcal{X}^{(b)}, \mathbb{Q}}^{\dagger} \rightarrow 0 .
$$

The construction of the left arrow of $(\star)$ is detailed just above (3.4.7.3) and the right arrow is given by the canonical connection of $\mathcal{D}_{\mathcal{X}}^{\dagger}\left(\right.$ b), $\mathbb{Q}$. As for (3.4.7.6), by applying the functor $g_{1 *}\left(-\otimes_{\mathcal{D}_{\mathcal{X}, \mathbb{Q}}^{\dagger}}^{\mathbb{L}} \mathcal{E}\right)\left(\right.$ resp. $g_{1 *}\left(-\otimes_{\mathcal{D}_{\mathcal{X}^{b}, \mathbb{Q}}^{\dagger}}^{\mathbb{L}}\right.$ $\mathcal{G})$ ) to the exact sequence $(\star)$ we get the isomorphism (3.5.4.1) (resp. (3.5.5.1)).

2) We now compute the isomorphism $\operatorname{coker}_{t_{1} \partial_{1}}(\mathcal{F}) \stackrel{\sim}{\rightarrow} \operatorname{coker}_{\partial_{1}}(\mathcal{E})$. We denote by $\delta_{\mathcal{X}^{b}}: \omega_{\mathcal{X}^{b}} \otimes_{\mathcal{O}_{\mathcal{X}}} \mathcal{D}_{\mathcal{X}^{b}, \mathbb{Q}}^{\dagger} \stackrel{\sim}{\rightarrow}$ $\omega_{\mathcal{X}^{b}} \otimes \mathcal{O}_{\mathcal{X}} \mathcal{D}_{\mathcal{X}^{b}, \mathbb{Q}}^{\dagger}$ as usual the involution isomorphism which exchanges both structure of right $\mathcal{D}_{\mathcal{X}^{b}, \mathbb{Q}^{-m o d u l e s}}^{\dagger}$ (this comes from [Car09a, 1.19] by completion and passage to the inductive limite). We get the isomorphism of right $\mathcal{D}_{\mathcal{X}^{b}, \mathbb{Q}^{-m o d u l e s: ~}}^{\dagger}$

$$
\Omega_{\mathcal{X}^{b} / \mathcal{Z}_{1}}^{1} \otimes_{\mathcal{O}_{\mathcal{X}}} \mathcal{D}_{\mathcal{X}^{b}, \mathbb{Q}}^{\dagger} \stackrel{\sim}{\rightarrow} g_{1}^{-1} \omega_{\mathcal{Z}_{1}}^{-1} \otimes_{g_{1}^{-1} \mathcal{O}_{\mathcal{Z}_{1}}}\left(\omega_{\mathcal{X}^{b}} \otimes_{\mathcal{O}_{\mathcal{X}}} \mathcal{D}_{\mathcal{X}^{b}, \mathbb{Q}}^{\dagger}\right) \stackrel{\sim}{\delta_{\mathcal{X}^{b}}}\left(\omega_{\mathcal{X}^{b}} \otimes_{\mathcal{O}_{\mathcal{X}}} \mathcal{D}_{\mathcal{X}^{b}, \mathbb{Q}}^{\dagger}\right) \otimes_{g_{1}^{-1} \mathcal{O}_{\mathcal{Z}_{1}}} g_{1}^{-1} \omega_{\mathcal{Z}_{1}}^{-1},
$$




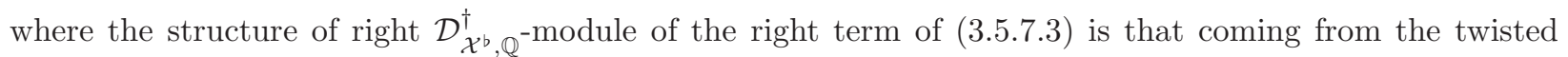
structure. From $\mathcal{D}_{\mathcal{X}^{b}, \mathbb{Q}}^{\dagger} \subset \mathcal{D}_{\mathcal{X}, \mathbb{Q}}^{\dagger}$, we get

$$
\left(\omega_{\mathcal{X}^{b}} \otimes_{\mathcal{O}_{\mathcal{X}}} \mathcal{D}_{\mathcal{X}^{b}, \mathbb{Q}}^{\dagger}\right) \otimes_{g_{1}^{-1} \mathcal{O}_{\mathcal{Z}_{1}}} g_{1}^{-1} \omega_{\mathcal{Z}_{1}}^{-1} \rightarrow\left(\omega_{\mathcal{X}^{b}} \otimes_{\mathcal{O}_{\mathcal{X}}} \mathcal{D}_{\mathcal{X}, \mathbb{Q}}^{\dagger}\right) \otimes_{g_{1}^{-1} \mathcal{O}_{\mathcal{Z}_{1}}} g_{1}^{-1} \omega_{\mathcal{Z}_{1}}^{-1}
$$

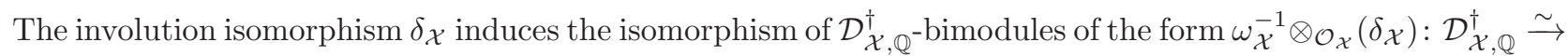

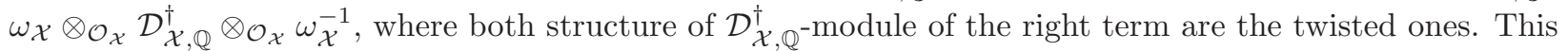

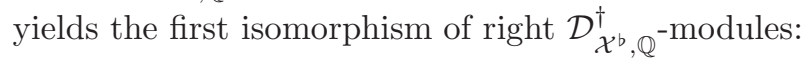

$$
\begin{aligned}
\left(\omega_{\mathcal{X}^{b}} \otimes_{\mathcal{O}_{\mathcal{X}}} \mathcal{D}_{\mathcal{X}, \mathbb{Q}}^{\dagger}\right) \otimes_{g_{1}^{-1} \mathcal{O}_{\mathcal{Z}_{1}}} g_{1}^{-1} \omega_{\mathcal{Z}_{1}}^{-1} \underset{\delta_{\mathcal{X}}}{\stackrel{\sim}{\longrightarrow}} g_{1}^{-1} \omega_{\mathcal{Z}_{1}}^{-1} \otimes_{g_{1}^{-1} \mathcal{O}_{\mathcal{Z}_{1}}} \otimes_{\mathcal{O}_{\mathcal{X}}} \omega_{\mathcal{X}} \otimes_{\mathcal{O}_{\mathcal{X}}}\left(\mathcal{D}_{\mathcal{X}, \mathbb{Q}}^{\dagger} \otimes_{\mathcal{O}_{\mathcal{X}}} \omega_{\mathcal{X}}^{-1} \otimes_{\mathcal{O}_{\mathcal{X}}} \omega_{\mathcal{X}^{b}}\right) \\
\stackrel{\sim}{\rightarrow} \Omega_{\mathcal{X} / \mathcal{Z}_{1}}^{1} \otimes_{\mathcal{O}_{\mathcal{X}}}\left(\mathcal{D}_{\mathcal{X}, \mathbb{Q}}^{\dagger} \otimes_{\mathcal{O}_{\mathcal{X}}} \omega_{\mathcal{X}}^{-1} \otimes_{\mathcal{O}_{\mathcal{X}}} \omega_{\mathcal{X}^{b}}\right)=\Omega_{\mathcal{X} / \mathcal{Z}_{1}}^{1} \otimes_{\mathcal{O}_{\mathcal{X}}} \mathcal{D}_{\mathcal{X} \leftarrow \mathcal{X}}^{\dagger}, \mathbb{Q}
\end{aligned}
$$

Hence, we get by composition of (3.5.7.3), (3.5.7.4) and (3.5.7.5) the morphism of right $\mathcal{D}_{\mathcal{X}^{b}}^{\dagger}, \mathbb{Q}^{-m o d u l e s: ~}$ $\alpha: \Omega_{\mathcal{X}^{b} / \mathcal{Z}_{1}}^{1} \otimes_{\mathcal{O}_{\mathcal{X}}} \mathcal{D}_{\mathcal{X}^{b}, \mathbb{Q}}^{\dagger} \rightarrow \Omega_{\mathcal{X} / \mathcal{Z}_{1}}^{1} \otimes_{\mathcal{O}_{\mathcal{X}}} \mathcal{D}_{\mathcal{X} \leftarrow \mathcal{X}^{b}, \mathbb{Q}}^{\dagger} . \quad$ Via $\mathcal{D}_{\mathcal{X} \leftarrow \mathcal{X}^{b}, \mathbb{Q}}^{\dagger} \stackrel{\sim}{\rightarrow} \mathcal{D}_{\mathcal{X}, \mathbb{Q}}^{\dagger} \otimes_{\mathcal{O}_{\mathcal{X}}} \mathcal{O}_{\mathcal{X}}\left(\mathcal{Z}_{1}\right) \subset \mathcal{D}_{\mathcal{X}}^{\dagger}\left({ }^{\dagger} Z_{1}\right)_{\mathbb{Q}}$, we will identify $\mathcal{D}_{\mathcal{X} \leftarrow \mathcal{X}^{b}, \mathbb{Q}}^{\dagger}$ with the sub $\left(\mathcal{D}_{\mathcal{X}, \mathbb{Q}}^{\dagger}, \mathcal{D}_{\mathcal{X}^{b}, \mathbb{Q}}^{\dagger}\right)$-bimodule $\mathcal{D}_{\mathcal{X}, \mathbb{Q}}^{\dagger} \frac{1}{t_{1}}$ of $\mathcal{D}_{\mathcal{X}}^{\dagger}\left({ }^{\dagger} Z_{1}\right)_{\mathbb{Q}}$. The inclusion $\mathcal{D}_{\mathcal{X}^{b}, \mathbb{Q}}^{\dagger} \subset$ $\mathcal{D}_{\mathcal{X}^{b}}^{\dagger}\left({ }^{\dagger} Z_{1}\right)_{\mathbb{Q}}=\mathcal{D}_{\mathcal{X}}^{\dagger}\left({ }^{\dagger} Z_{1}\right)_{\mathbb{Q}}$ has the factorisation $\iota: \mathcal{D}_{\mathcal{X}^{b}, \mathbb{Q}}^{\dagger} \subset \mathcal{D}_{\mathcal{X} \leftarrow \mathcal{X}^{b}, \mathbb{Q}}^{\dagger}$. Consider now the following diagram of right $\mathcal{D}_{\mathcal{X}^{b}, \mathbb{Q}^{-m o d u l e s}}^{\dagger}$

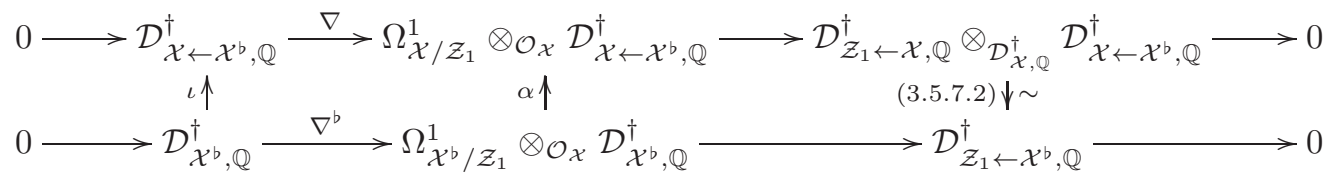

where the sequence of the bottom (resp. of the top) is the respective case of $(\star)$ (resp. is the image of $(\star)$ under the functor $\left.-\otimes_{\mathcal{D}_{\mathcal{X}, \mathbb{Q}}^{\dagger}} \mathcal{D}_{\mathcal{X} \leftarrow \mathcal{X}^{b}, \mathbb{Q}}^{\dagger}\right)$. The exactness of the sequence of the bottom has already been checked. Concerning that of the top of (3.5.7.6), using the vanishing property given by (3.5.7.2), this comes from that of $(\star)$. By identifying $\mathcal{D}_{\mathcal{X} \leftarrow \mathcal{X}^{b}, \mathbb{Q}}^{\dagger}$ with the sub $\left(\mathcal{D}_{\mathcal{X}, \mathbb{Q}}^{\dagger}, \mathcal{D}_{\mathcal{X}^{b}, \mathbb{Q}}^{\dagger}\right)$-bimodule $\mathcal{D}_{\mathcal{X}, \mathbb{Q}}^{\dagger} \frac{1}{t_{1}}$ of $\mathcal{D}_{\mathcal{X}}^{\dagger}\left({ }^{\dagger} Z_{1}\right)_{\mathbb{Q}}$, we compute that $\alpha$ is such that $d \log t_{1} \otimes 1 \mapsto d t_{1} \otimes \frac{1}{t_{1}}$ and is determined by $\mathcal{D}_{\mathcal{X}^{b}, \mathbb{Q}^{-}}^{\dagger}$ linearity by

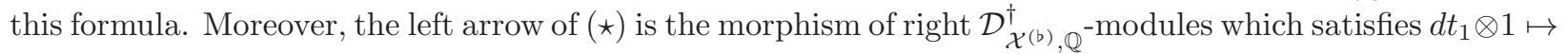
$g_{1}^{*}(1) \otimes\left(d t_{1} \wedge \cdots \wedge d t_{d}\right) \otimes\left(d t_{2} \wedge \cdots \wedge d t_{d}\right)^{*}\left(\right.$ resp. $d \log _{1} \otimes 1 \mapsto g_{1}^{*}(1) \otimes\left(d \log _{1} \wedge d t_{2} \wedge \cdots \wedge d t_{d}\right) \otimes\left(d t_{2} \wedge \cdots \wedge d t_{d}\right)^{*}$. Finally, we compute that the isomorphism (3.5.7.2) sends $\left(g_{1}^{*}(1) \otimes\left(d t_{1} \wedge \cdots \wedge d t_{d}\right) \otimes\left(d t_{2} \wedge \cdots \wedge d t_{d}\right)^{*}\right) \otimes \frac{1}{t_{1}}$ to $g_{1}^{*}(1) \otimes\left(d \log t_{1} \wedge d t_{2} \wedge \cdots \wedge d t_{d}\right) \otimes\left(d t_{2} \wedge \cdots \wedge d t_{d}\right)^{*}$. Hence, we compute that the diagram (3.5.7.6) is commutative.

The commutativity of diagram of modules (3.5.7.6) can be formulated as follows: we have the commutative diagram of complexes:

$$
\begin{gathered}
{\left[\mathcal{D}_{\mathcal{X} \leftarrow \mathcal{X}^{b}, \mathbb{Q}}^{\dagger} \stackrel{\nabla}{\longrightarrow} \Omega_{\mathcal{X} / \mathcal{Z}_{1}}^{1} \otimes_{\mathcal{O}_{\mathcal{X}}} \mathcal{D}_{\mathcal{X} \leftarrow \mathcal{X}^{b}, \mathbb{Q}}^{\dagger}\right] \longrightarrow \mathcal{D}_{\mathcal{Z}_{1} \leftarrow \mathcal{X}, \mathbb{Q}}^{\dagger} \otimes_{\mathcal{D}_{\mathcal{X}, \mathbb{Q}}^{\dagger}} \mathcal{D}_{\mathcal{X} \leftarrow \mathcal{X}^{b}, \mathbb{Q}}^{\dagger}} \\
\sim \uparrow(3.5 .7 .2) \\
{\left[\mathcal{D}_{\mathcal{X}^{b}, \mathbb{Q}}^{\dagger} \stackrel{\nabla^{b}}{\longrightarrow} \Omega_{\mathcal{X}^{b} / \mathcal{Z}_{1}}^{1} \otimes_{\mathcal{O}_{\mathcal{X}}} \mathcal{D}_{\mathcal{X}^{b}, \mathbb{Q}}^{\dagger}\right] \longrightarrow \mathcal{D}_{\mathcal{Z}_{1} \leftarrow \mathcal{X}^{b}, \mathbb{Q}}^{\dagger},}
\end{gathered}
$$

where the left arrow is given by $\iota$ and $\alpha$ as above. By applying the functor $g_{1 *}\left(-\otimes_{\mathcal{D}_{\mathcal{X}^{b}}^{\dagger}}^{\mathbb{L}} \mathcal{F}\right)$ to the diagram 
(3.5.7.7), since $u_{+}(\mathcal{F})=\mathcal{D}_{\mathcal{X} \leftarrow \mathcal{X}^{b}, \mathbb{Q}}^{\dagger} \otimes_{\mathcal{D}_{\mathcal{X}^{b}, \mathbb{Q}}^{\dagger}}^{\mathbb{L}} \mathcal{F}$, we get the commutative diagram of complexes:

$$
\begin{array}{r}
g_{1 *}\left[u_{+}(\mathcal{F}) \stackrel{\nabla}{\longrightarrow} \Omega_{\mathcal{X} / \mathcal{Z}_{1}} \otimes_{\mathcal{O}_{\mathcal{X}}} u_{+}(\mathcal{F})\right] \underset{(3.5 .4 .1)}{\stackrel{\sim}{\longrightarrow}} g_{1 *}\left(\mathcal{D}_{\mathcal{Z}_{1} \leftarrow \mathcal{X}}^{\dagger} \otimes_{\mathcal{D}_{\mathcal{X}}^{\dagger}}^{\mathbb{L}} u_{+}(\mathcal{F})\right) \\
\uparrow \\
g_{1 *}\left[\mathcal{F} \stackrel{\nabla^{b}}{\longrightarrow} \Omega_{\mathcal{X}^{b} / \mathcal{Z}_{1}} \otimes_{\mathcal{O}_{\mathcal{X}}} \mathcal{F}\right] \underset{(3.5 .7 .1)}{\stackrel{\sim}{(3.5 .5 .1)}} g_{1 *}\left(\mathcal{D}_{\mathcal{Z}_{1} \leftarrow \mathcal{X}^{b}}^{\dagger} \otimes_{\mathcal{D}_{\mathcal{X}^{b}}^{\dagger}}^{\mathbb{L}} \mathcal{F}\right),
\end{array}
$$

where the left morphism is in fact (by acyclicity) the morphism of complexes which is the image under the functor $g_{1 *}\left(-\otimes_{\mathcal{D}_{\mathcal{X}^{b}}^{\dagger}} \mathcal{F}\right)$ of the left morphism of (3.5.7.7). From the commutativity of (3.5.7.8), by definition of the isomorphism (3.5.6.1), we get that the morphism of complexes of the left of (3.5.7.8) is precisely the isomorphism (3.5.6.1).

Now, let us describe the left morphism of (3.5.7.8). By forgetting to mention $g_{1 *}$, this left morphism of (3.5.6.1) is given by two (compatible with $\nabla$ and $\nabla^{b}$ ) morphisms, one is of the form $\mathcal{F} \rightarrow \mathcal{E}$ and the other one is of the form $\Omega_{\mathcal{X}^{b} / \mathcal{Z}_{1}} \otimes_{\mathcal{O}_{\mathcal{X}}} \mathcal{F} \rightarrow \Omega_{\mathcal{X} / \mathcal{Z}_{1}} \otimes_{\mathcal{O}_{\mathcal{X}}} \mathcal{E}$ (recall that $u_{+}(\mathcal{F})=\mathcal{E}$ ). By using the canonical isomorphism $\mathcal{F}\left({ }^{\dagger} Z_{1}\right) \stackrel{\sim}{\rightarrow} u_{+}(\mathcal{F})$, the first morphism is given by the inclusion $\mathcal{F} \hookrightarrow \mathcal{F}\left({ }^{\dagger} Z_{1}\right) \stackrel{\sim}{\rightarrow} u_{+}(\mathcal{F})=\mathcal{E}$ (indeed, the morphism is induced by the inclusion $\iota$ ). By identifying $\Omega_{\mathcal{X} / \mathcal{Z}_{1}}^{1}\left(\right.$ resp. $\left.\Omega_{\mathcal{X}^{b} / \mathcal{Z}_{1}}^{1}\right)$ with $\mathcal{O}_{\mathcal{X}}$ via the basis $d t_{1}$ (resp. via tha basis $d \log _{1}$ ), we compute that the second morphism is given by the multiplication by $\frac{1}{t_{1}}: \mathcal{F} \hookrightarrow \mathcal{F}\left({ }^{\dagger} Z_{1}\right) \stackrel{\sim}{\rightarrow} u_{+}(\mathcal{F})=\mathcal{E}$ (see the computation describing $\alpha$ above). This yields that the left morphism of (3.5.7.8) can be identified with the left square of the diagram:

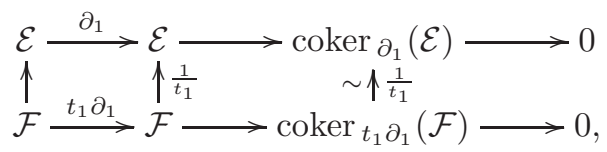

The right (vertical) morphism of the diagram (3.5.7.9) is then equal to the isomorphism of the proposition 3.5.7.

3) Finally we check the compatibility with Frobenius. Consider the diagram

$$
\begin{gathered}
F_{\mathcal{Z}_{1}}^{*} \operatorname{coker}_{\partial_{1}}(\mathcal{E}) \stackrel{\sim}{\longrightarrow} \operatorname{coker}_{\partial_{1}^{\prime}}\left(\mathcal{E}^{\prime}\right) \stackrel{\sim}{\longrightarrow} \operatorname{coker}_{\partial_{1}}\left(F_{\mathcal{X}}^{*} \mathcal{E}\right) \underset{t_{1}}{\stackrel{\sim}{t_{1}^{\prime}}} \stackrel{\sim}{\longrightarrow} \operatorname{coker}_{\partial_{1}}(\mathcal{E}) \\
F_{\mathcal{Z}_{1}}^{*} \operatorname{coker}_{t_{1} \partial_{1}}(\mathcal{F}) \stackrel{\uparrow \frac{1}{t_{1}}}{\sim} \operatorname{coker}_{t_{1}^{\prime} \partial_{1}^{\prime}}\left(\mathcal{F}^{\prime}\right) \stackrel{\sim}{\longrightarrow} \operatorname{coker}_{t_{1} \partial_{1}}\left(F_{\mathcal{X}}^{*} \mathcal{F}\right) \underset{\phi_{\mathcal{F}}}{\sim} \operatorname{coker}_{t_{1} \partial_{1}}(\mathcal{F}),
\end{gathered}
$$

where the composition of the top is the isomorphism $F_{\mathcal{Z}_{1}}^{*}$ coker $\partial_{1}(\mathcal{E}) \rightarrow$ coker $\partial_{1}(\mathcal{E})$ is the Frobenius structure of $\operatorname{coker}_{\partial_{1}}(\mathcal{E})$ as in (3.5.4.4) and the isomorphism below $F_{\mathcal{Z}_{1}}^{*} \operatorname{coker}_{t_{1} \partial_{1}}(\mathcal{F}) \stackrel{\sim}{\rightarrow} \operatorname{coker}_{t_{1} \partial_{1}}(\mathcal{F})$ is the Frobenius as defined in (3.5.5.4). By using the commutativity of (3.5.2.1) and the functoriality in $\mathcal{F}$ of the diagram (3.5.7.9) (i.e. replacing $\mathcal{F}$ by a morphism $\mathcal{F}_{1} \rightarrow \mathcal{F}_{2}$ and $\mathcal{E}$ by $u_{+}\left(\mathcal{F}_{1}\right) \rightarrow u_{+}\left(\mathcal{F}_{2}\right)$ we get a similar diagram in three dimension), we get the commutativity of the right square of (3.5.7.10). The commutativity of the left square of (3.5.7.10) is obvious. Since $F_{\mathcal{X} / \mathcal{Z}_{1}}^{*}: t_{1}^{\prime} \mapsto t_{1}^{q}$, using the computation of the horizontal morphisms of the middle square (see respectively just after (3.5.4.3) and (3.5.5.3)), we get the commutativity of the middle square. Hence, the diagram (3.5.7.10) is commutative, which finishes the proof.

3.5.8. Since $N_{1, \mathcal{F}}: \mathcal{H}(1) \rightarrow \mathcal{H}$ commutes with Frobenius, we get Frobenius structures on ker $N_{1, \mathcal{F}}$ and coker $N_{1, \mathcal{F}}$. Moreover, by applying the functor coker $t_{1} \partial_{1}$ to the surjection $\mathcal{F} \rightarrow \mathcal{F} / \mathcal{F}\left(-\mathcal{Z}_{1}\right)$, we get the morphism $\vartheta_{\mathcal{F}}: \operatorname{coker}_{t_{1} \partial_{1}}(\mathcal{F})(1) \rightarrow \operatorname{coker} N_{1, \mathcal{F}}$.

Lemma 3.5.9. The canonical morphism $\vartheta_{\mathcal{F}}$ : $\operatorname{coker}_{t_{1} \partial_{1}}(\mathcal{F})(1) \rightarrow$ coker $N_{1, \mathcal{F}}$ defined in 3.5.8 is compatible with Frobenius (for the respective Frobenius structures defined in (3.5.5.4) and 3.5.8). 
Proof. Consider the diagram:

$$
\begin{aligned}
& F_{\mathcal{Z}_{1}}^{*} \operatorname{coker}_{t_{1} \partial_{1}}(\mathcal{F}) \stackrel{\sim}{\longrightarrow} \operatorname{coker}_{t_{1}^{\prime} \partial_{1}^{\prime}}\left(\mathcal{F}^{\prime}\right) \underset{q \cdot \operatorname{adj}}{\sim} \operatorname{coker}_{t_{1} \partial_{1}}\left(F_{\mathcal{X}}^{*} \mathcal{F}\right) \underset{\phi_{\mathcal{F}}}{\stackrel{\sim}{\longrightarrow}} \operatorname{coker}_{t_{1} \partial_{1}}(\mathcal{F}) \\
& \downarrow q F_{\mathcal{Z}_{1}}^{*} \vartheta_{\mathcal{F}} \quad \downarrow \vartheta_{F_{\mathcal{X}} \mathcal{F}} \sim \phi_{\mathcal{F}} \quad \downarrow \vartheta_{\mathcal{F}} \\
& F_{\mathcal{Z}_{1}}^{*} \operatorname{coker} N_{1, \mathcal{F}} \sim \operatorname{coker}\left(N_{1, F_{\mathcal{X}}^{*} \mathcal{F}} \underset{\phi_{\mathcal{H}}}{\stackrel{\sim}{\longrightarrow}} \operatorname{coker} N_{1, \mathcal{F}},\right.
\end{aligned}
$$

where the composition of the top horizontal morphisms is the Frobenius structure as defined in (3.5.5.4) and where the composition of the bottom horizontal morphisms is the Frobenius structure of coker $\left(N_{1, \mathcal{F}}\right)$. The right square is commutative by functoriality of $\mathcal{F} \mapsto \vartheta_{\mathcal{F}}$ (and by definition of $\phi_{\mathcal{H}}$ given in (3.2.11.2)). We check the commutativity of the left triangle by computation.

Lemma 3.5.10. Consider the commutative square of coherent $\mathcal{D}_{\mathcal{Z}_{1}, \mathbb{Q}^{-m o d u l e s}}^{\dagger}$

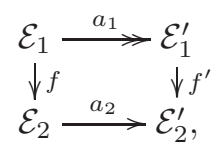

whose homomorphism on the top is surjective. We suppose these modules have a Frobenius structure such that $f, a_{1}, a_{2}$ commute with Frobenius. Then so is $f^{\prime}$.

Proof. It is sufficient to check that two morphisms of the form $F_{\mathcal{Z}_{1}}^{*} \mathcal{E}_{1}^{\prime} \rightarrow \mathcal{E}_{2}^{\prime}$ which are equal after composition with $F_{\mathcal{Z}_{1}}^{*}\left(a_{1}\right): F_{\mathcal{Z}_{1}}^{*} \mathcal{E}_{1} \rightarrow F_{\mathcal{Z}_{1}}^{*} \mathcal{E}_{1}^{\prime}$ are equal. This property is satisfied because $F_{\mathcal{Z}_{1}}^{*}\left(a_{1}\right)$ is surjective.

Proposition 3.5.11. The isomorphisms

$$
\operatorname{ker}\left(N_{1, \mathcal{F}}\right) \stackrel{\sim}{\rightarrow} g_{1+} \operatorname{ker}\left(\theta_{\mathcal{E}}\right), \quad \operatorname{coker}\left(N_{1, \mathcal{F}}\right) \stackrel{\sim}{\rightarrow} g_{1+} \operatorname{coker}\left(\theta_{\mathcal{E}}\right),
$$

which are constructed by composing (3.4.17.1) and (3.4.16.2), commute with Frobenius.

Proof. First, let us prove the compatibility with Frobenius of the second isomorphism. Consider the following diagram:

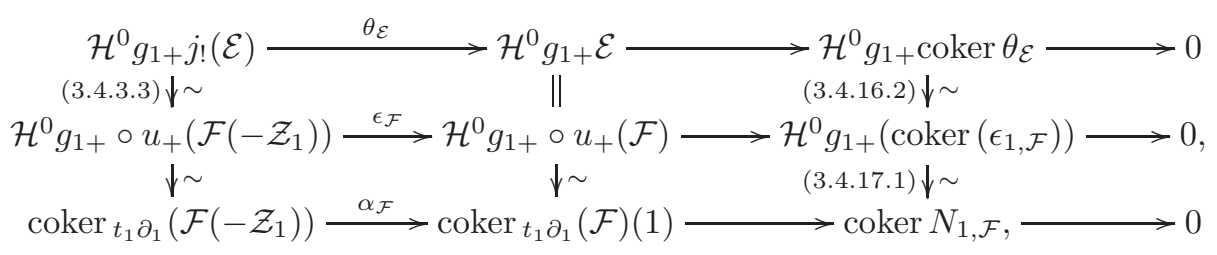

where the left above square is (modulo the biduality isomorphism $\mathbb{D}_{\mathcal{X}} \circ \mathbb{D}_{\mathcal{X}}(\mathcal{E}) \stackrel{\sim}{\rightarrow} \mathcal{E}$ ) the image under the functor $\mathcal{H}^{0} g_{1}$ of the square (3.4.16.1), where both vertical morphisms of the bottom left square comes from the canonical isomorphisms $\mathcal{H}^{0} g_{1+} \circ u_{+} \stackrel{\sim}{\rightarrow} \mathcal{H}^{0} g_{1+}^{b} \underset{(3.5 .5 .2)}{\stackrel{\sim}{\longrightarrow}}$ coker $_{t_{1} \partial_{1}}$. Both left squares are commutative by definition or fonctoriality. The right square of the top is commutative by definition of the isomorphism (3.4.16.2). Using the remark 3.4.18, we check the commutativity of the the bottom right square. Hence, the diagram is commutative.

Since $\mathcal{X}$ is affine, the functors coker $t_{1} \partial_{1}$ and $\mathcal{H}^{0} g_{1+}$ are right exact, so the horizontal sequences are exact. Now, the horizontal morphisms on the top commute with Frobenius. The composition of the middle vertical homomorphisms $\mathcal{H}^{0} g_{1+} \mathcal{E} \stackrel{\sim}{\rightarrow} \operatorname{coker}_{t_{1} \partial_{1}}(\mathcal{F})(1)$ is compatible with Frobenius by (3.5.4.2) and Lemma 3.5.7, and the right horizontal homomorphism on the bottom as well by Lemma 3.5.9. We conclude the proof by using Lemma 3.5.10.

Finally we get the compatibility with Frobenius of the first isomorphism of 3.5.11 from that of the second one via the isomorphisms:

$$
\begin{aligned}
& \mathbb{D}_{\mathcal{X}} i_{1+} \operatorname{ker} N_{1, \mathcal{F}} \stackrel{\sim}{\rightarrow} i_{1+} \mathbb{D}_{\mathcal{Z}_{1}} \operatorname{ker} N_{1, \mathcal{F}} \underset{[\mathrm{Abe13}, 3.12]}{\stackrel{\sim}{\longrightarrow}} i_{1+}\left(\operatorname{ker} N_{1, \mathcal{F}}\right)^{\vee}(-d+1) \underset{(3.2 .5 .1)}{\stackrel{\sim}{\rightarrow}} i_{1+} \operatorname{coker}\left(N_{1, \mathcal{F}}^{\vee}\right)(-d+1) \\
& \underset{3.2 .3}{\stackrel{\sim}{\longrightarrow}} i_{1+} \operatorname{coker}\left(N_{1, \mathcal{F}^{\vee}}\right)(-d) \stackrel{\sim}{\rightarrow} \operatorname{coker}\left(\theta_{\mathcal{E}^{\vee}}\right)(-d) \underset{[\operatorname{Abe13}, 3.12]}{\stackrel{\sim}{\longrightarrow}} \operatorname{coker}\left(\theta_{\mathbb{D}_{\mathcal{X}, Z} \mathcal{E}}\right) \underset{1.4 .3 .1}{\stackrel{\sim}{\longrightarrow}} \mathbb{D}_{\mathcal{X}} \operatorname{ker}\left(\theta_{\mathcal{E}} \cdot\right)
\end{aligned}
$$


Lemma 3.5.12. Let $(Y, X, \mathcal{P})$ be a frame, and $j=(\star, \mathrm{id}, \mathrm{id}):(Y, X, \mathcal{P}) \rightarrow(X, X, \mathcal{P})$ be a morphism of frames. Let $\mathcal{G}_{1} \in F-\mathrm{Ovhol}(X, \mathcal{P} / K), \mathcal{G}_{2} \in F-\operatorname{Ovhol}(Y, \mathcal{P} / K)$, and $\phi: \mathcal{G}_{1} \stackrel{\sim}{\rightarrow} j_{!}\left(\mathcal{G}_{2}\right)$ be an isomorphism in $\operatorname{Ovhol}(X, \mathcal{P} / K)$. Let $\mathcal{U}$ be an open formal subscheme of $\mathcal{P}$ containing $Y$. If $\left.\phi\right|_{\mathcal{U}}$ commutes with the Frobenius structures, then so is $\phi$.

Proof. Let us show that $\phi^{-1}$ is compatible with Frobenius. By taking the adjoint, it suffices to show that the homomorphism $j^{!}\left(\phi^{-1}\right): \mathcal{G}_{2} \rightarrow j^{!} \mathcal{G}_{1}$ in $\operatorname{Ovhol}(Y, \mathcal{P})$ is compatible with Frobenius. The lemma follows since the restriction functor $\operatorname{Ovhol}(Y, \mathcal{P}) \rightarrow \operatorname{Ovhol}(Y, \mathcal{U})$ is faithful by Remark 1.2.10.

Theorem 3.5.13. We consider the situation in paragraph 3.4.19, and $D_{1}$ is not empty anymore. The isomorphisms (3.4.19.1) are compatible with Frobenius.

Proof. From Lemma 3.5.12, it is enough to check it outside $D_{1}$, and we can suppose $D_{1}$ to be empty. By the same reason given at the first step of the proof of Lemma 3.4.11, we can suppose $f=$ id. Since by construction, outside of $D_{1}$, the isomorphisms of (3.4.19.1) are (modulo the biduality isomorphism) that constructed in (3.4.19.2), i.e. are equal to that of Proposition 3.5.11, then the claim follows by this proposition 3.5.11.

\subsection{Stability of mixedness for a unipotent $F$-isocrystal}

Let $(Y, X, \mathcal{P})$ be a frame such that $X$ is smooth and there exists a strict normal crossing divisor $Z$ of $X$ such that $Y:=X \backslash Z$. Let $\left\{Z_{i}\right\}_{i \in I}$ be the set of irreducible components of $Z, r:=\# I$. We can suppose $I=\{1, \ldots, r\}$. For $J \subset I$, we put $Z_{J}:=\bigcap_{i \in J} Z_{i}$. Then we define

$$
Z_{(0)}:=X, \quad Z_{(k)}:=\bigcup_{\# J=k} Z_{J}, \quad Z_{(k)}^{\circ}:=Z_{(k)} \backslash Z_{(k+1)} .
$$

Then $\left\{Z_{(k)}^{\circ}\right\}_{0 \leq k \leq r}$ is a smooth stratification of $X$. This stratification is denoted by $\operatorname{Strat}_{Z}(X)$.

Lemma 3.6.1 (Gluing isomorphisms). We suppose $p^{m}>e / p-1$, where e is the absolute ramification index of $\mathcal{V}$. Let $f, g, h: X \rightarrow Y$ be a morphism of log-smooth log-schemes over $V / \pi^{i+1} \mathcal{V}$ and $f_{0}, g_{0}, h_{0}: X_{0} \rightarrow Y_{0}$ the induced morphism of $\log$-schemes over $V / \pi^{1} \mathcal{V}$. Let $\mathcal{F}$ be a left $\mathcal{D}_{Y}^{(m)}$-module. If $f_{0}=g_{0}=h_{0}$, then there exists a canonical morphism of $\mathcal{D}_{X}^{(m)}$-modules

$$
\tau_{f, g}: g^{*} \mathcal{F} \stackrel{\sim}{\rightarrow} f^{*} \mathcal{F}
$$

such that $\tau_{f, f}=\mathrm{Id}$ and $\tau_{f, h}=\tau_{f, g} \circ \tau_{g, h}$.

Proof. Since $p^{m}>e / p-1, \mathfrak{a}:=\pi \mathcal{O}_{T}$ is endowed with a canonical $m$-PD-structure which is $m$-PD-nilpotente (see [Ber00, A.4]). Hence, we can copy the proof of [Ber00, 2.1.5].

3.6.2. Let $\left(\mathcal{P}_{\alpha}\right)_{\alpha \in \Lambda}$ be a cover of $\mathcal{P}$ by affine open sets $\mathcal{P}_{\alpha}$ endowed with local coordinates $t_{1}^{\alpha}, \ldots, t_{n}^{\alpha}$ such that $\bar{t}_{1}^{\alpha}, \ldots, \bar{t}_{d}^{\alpha}$ are local coordinates of $X_{\alpha}:=X \cap P_{\alpha}\left(\right.$ where $\bar{t}_{i}^{\alpha}$ is the image of $t_{i}^{\alpha}$ via $\Gamma\left(\mathcal{P}, \mathcal{O}_{\mathcal{P}_{\alpha}}\right) \rightarrow \Gamma\left(X_{\alpha}, \mathcal{O}_{X_{\alpha}}\right)$ ) and such that $Z_{i} \cap X_{\alpha}=V\left(\bar{t}_{i}^{\alpha}\right)$.

We set $\mathcal{P}_{\alpha \beta}:=\mathcal{P}_{\alpha} \cap \mathcal{P}_{\beta}, \mathcal{P}_{\alpha \beta \gamma}:=\mathcal{P}_{\alpha} \cap \mathcal{P}_{\beta} \cap \mathcal{P}_{\gamma}, X_{\alpha}:=X \cap P_{\alpha}, X_{\alpha \beta}:=X_{\alpha} \cap X_{\beta}$ et $X_{\alpha \beta \gamma}:=X_{\alpha} \cap X_{\beta} \cap X_{\gamma}$. We denote by $Y_{\alpha}$ the open of $X_{\alpha}$ complementary to $Z, Y_{\alpha \beta}:=Y_{\alpha} \cap Y_{\beta}, Y_{\alpha \beta \gamma}:=Y_{\alpha} \cap Y_{\beta} \cap Y_{\gamma}, j_{\alpha}: Y_{\alpha} \hookrightarrow X_{\alpha}$, $j_{\alpha \beta}: Y_{\alpha \beta} \hookrightarrow X_{\alpha \beta}$ and $j_{\alpha \beta \gamma}: Y_{\alpha \beta \gamma} \hookrightarrow X_{\alpha \beta \gamma}$ the canonical open immersions.

For any 3uple $(\alpha, \beta, \gamma) \in \Lambda^{3}$, fix $\mathcal{X}_{\alpha}\left(\right.$ resp. $\left.\mathcal{X}_{\alpha \beta}, \mathcal{X}_{\alpha \beta \gamma}\right)$ some smooth formal $\mathcal{V}$-schemes lifting $X_{\alpha}$ (resp. $\left.X_{\alpha \beta}, X_{\alpha \beta \gamma}\right)$. Choose etale morphisms $\phi_{\alpha}: \mathcal{X}_{\alpha} \rightarrow \widehat{\mathbb{A}}_{\mathcal{V}}^{d}, \phi_{\alpha, \beta}: \mathcal{X}_{\alpha, \beta} \rightarrow \widehat{\mathbb{A}}_{\mathcal{V}}^{d}, \phi_{\alpha, \beta, \gamma}: \mathcal{X}_{\alpha, \beta, \gamma} \rightarrow \widehat{\mathbb{A}}_{\mathcal{V}}^{d}$. We denote by $\mathcal{Z}_{\alpha}:=\phi_{\alpha}^{-1}\left(V\left(x_{1} \cdots x_{d}\right)\right), \mathcal{Z}_{\alpha, \beta}:=\phi_{\alpha, \beta}^{-1}\left(V\left(x_{1} \cdots x_{d}\right)\right), \mathcal{Z}_{\alpha, \beta, \gamma}:=\phi_{\alpha, \beta, \gamma}^{-1}\left(V\left(x_{1} \cdots x_{d}\right)\right)$, where $x_{1}, \ldots, x_{r}$ are the $r$ first coordinates of $\widehat{\mathbb{A}}_{\mathcal{V}}^{d}$. We can suppose that $Z_{\alpha}:=X_{\alpha} \cap Z, Z_{\alpha, \beta}=X_{\alpha, \beta} \cap Z$ and $Z_{\alpha, \beta, \gamma}=X_{\alpha, \beta, \gamma} \cap Z$. We set $\mathcal{X}_{\alpha}^{b}:=\left(\mathcal{X}_{\alpha}, \mathcal{Z}_{\alpha}\right), \mathcal{X}_{\alpha \beta}^{b}:=\left(\mathcal{X}_{\alpha \beta}, \mathcal{Z}_{\alpha \beta}\right), \mathcal{X}_{\alpha \beta \gamma}^{b}:=\left(\mathcal{X}_{\alpha \beta \gamma}, \mathcal{Z}_{\alpha \beta, \gamma}\right)$. 
Using [Kat89, 3.11], there exists a morphism of formal $\mathcal{V}$-schemes of the form $p_{1}^{\alpha \beta b}: \mathcal{X}_{\alpha \beta}^{b} \rightarrow \mathcal{X}_{\alpha}^{b}$ (resp. $\left.p_{2}^{\alpha \beta b}: \mathcal{X}_{\alpha \beta}^{b} \rightarrow \mathcal{X}_{\beta}^{b}\right)$ which is a lifting of $X_{\alpha \beta}^{b} \rightarrow X_{\alpha}^{b}\left(\right.$ resp. $\left.X_{\alpha \beta}^{b} \rightarrow X_{\beta}^{b}\right)$. Similarly, for any $(\alpha, \beta, \gamma) \in$ $\Lambda^{3}$, fix some lifting $p_{12}^{\alpha \beta \gamma b}: \mathcal{X}_{\alpha \beta \gamma}^{b} \rightarrow \mathcal{X}_{\alpha \beta}^{b}, p_{23}^{\alpha \beta \gamma b}: \mathcal{X}_{\alpha \beta \gamma}^{b} \rightarrow \mathcal{X}_{\beta \gamma}^{b}, p_{13}^{\alpha \beta \gamma b}: \mathcal{X}_{\alpha \beta \gamma}^{b} \rightarrow \mathcal{X}_{\alpha \gamma}^{b}, p_{1}^{\alpha \beta \gamma b}: \mathcal{X}_{\alpha \beta \gamma}^{b} \rightarrow \mathcal{X}_{\alpha}^{b}$, $p_{2}^{\alpha \beta \gamma b}: \mathcal{X}_{\alpha \beta \gamma}^{b} \rightarrow \mathcal{X}_{\beta}^{b}, p_{3}^{\alpha \beta \gamma b}: \mathcal{X}_{\alpha \beta \gamma}^{b} \rightarrow \mathcal{X}_{\gamma}^{b}$. By removing the symbol "b" we mean the corresponding formal scheme or morphism of formal schemes.

Definition 3.6.3. We keep notation 3.6.2. We define the category $F-\operatorname{Isoc}\left(\left(\mathcal{X}_{\alpha}^{b}\right)_{\alpha \in \Lambda} / K\right)$ as follows:

- an object is a family $\left(\mathcal{F}_{\alpha}\right)_{\alpha \in \Lambda}$ of coherent $\mathcal{D}_{\mathcal{X}_{\alpha}^{b}, \mathbb{Q}^{-}}^{\dagger}$-modules which are locally projective of finite type as $\mathcal{O}_{\mathcal{X}_{\alpha}, \mathbb{Q}}$-module and endowed with a glueing data $\left(\vartheta_{\alpha \beta}\right)_{\alpha, \beta \in \Lambda}$, i.e. the data for any $\alpha, \beta \in \Lambda$ of a $\mathcal{D}_{\mathcal{X}_{\alpha \beta}^{b}, \mathbb{Q}^{-}}^{\text {-linear }}$ isomorphism $\vartheta_{\alpha \beta}: p_{2}^{\alpha \beta b !}\left(\mathcal{F}_{\beta}\right) \stackrel{\sim}{\rightarrow} p_{1}^{\alpha \beta b !}\left(\mathcal{F}_{\alpha}\right)$, satisfying the cocycle condition: $\vartheta_{13}^{\alpha \beta \gamma}=\vartheta_{12}^{\alpha \beta \gamma} \circ \vartheta_{23}^{\alpha \beta \gamma}$, where $\vartheta_{12}^{\alpha \beta \gamma}, \vartheta_{23}^{\alpha \beta \gamma}$ and $\vartheta_{13}^{\alpha \beta \gamma}$ are defined via the commutative squares

$$
\begin{aligned}
& p_{12}^{\alpha \beta \gamma b !} p_{2}^{\alpha \beta b !}\left(\mathcal{F}_{\beta}\right) \stackrel{\tau}{\underset{\sim}{\longrightarrow}} p_{2}^{\alpha \beta \gamma b !}\left(\mathcal{F}_{\beta}\right) \quad p_{23}^{\alpha \beta \gamma b !} p_{2}^{\beta \gamma b !}\left(\mathcal{F}_{\gamma}\right) \stackrel{\tau}{\underset{\sim}{\longrightarrow}} p_{3}^{\alpha \beta \gamma b !}\left(\mathcal{F}_{\gamma}\right) \quad p_{13}^{\alpha \beta \gamma b !} p_{2}^{\alpha \gamma b !}\left(\mathcal{F}_{\gamma}\right) \stackrel{\tau}{\sim} p_{3}^{\alpha \beta \gamma b !}\left(\mathcal{F}_{\gamma}\right)
\end{aligned}
$$

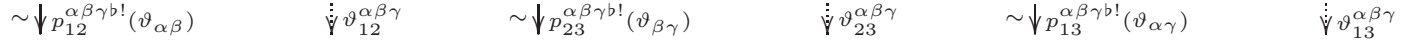

$$
\begin{aligned}
& p_{12}^{\alpha \beta \gamma b !} p_{1}^{\alpha \beta b !}\left(\mathcal{F}_{\alpha}\right) \stackrel{\tau}{\sim} p_{1}^{\alpha \beta \gamma b !}\left(\mathcal{F}_{\alpha}\right), p_{23}^{\alpha \beta \gamma b !} p_{1}^{\beta \gamma b !}\left(\mathcal{F}_{\beta}\right) \stackrel{\tau}{\sim} p_{2}^{\alpha \beta \gamma b !}\left(\mathcal{F}_{\beta}\right), p_{13}^{\alpha \beta \gamma b !} p_{1}^{\alpha \gamma b !}\left(\mathcal{F}_{\alpha}\right) \stackrel{\tau}{\sim} p_{1}^{\alpha \beta \gamma b !}\left(\mathcal{F}_{\alpha}\right) \text {, }
\end{aligned}
$$

where the glueing isomorphisms denoted by $\tau$ are defined from 3.6.1 by completion, tensorising by $\mathbb{Q}$ and inductive limits.

- a morphism $\left(\left(\mathcal{F}_{\alpha}\right)_{\alpha \in \Lambda},\left(\vartheta_{\alpha \beta}\right)_{\alpha, \beta \in \Lambda}\right) \rightarrow\left(\left(\mathcal{F}_{\alpha}^{\prime}\right)_{\alpha \in \Lambda},\left(\vartheta_{\alpha \beta}^{\prime}\right)_{\alpha, \beta \in \Lambda}\right)$ is a familly of morphisms $f_{\alpha}: \mathcal{F}_{\alpha} \rightarrow \mathcal{F}_{\alpha}^{\prime}$ commuting with glueing datas, i.e., such that the following diagrams are commutative :

$$
\begin{gathered}
p_{2}^{\alpha \beta b !}\left(\mathcal{F}_{\beta}\right) \stackrel{\vartheta_{\alpha \beta}}{\sim} p_{1}^{\alpha \beta b !}\left(\mathcal{F}_{\alpha}\right) \\
\downarrow p_{2}^{\alpha \beta b !}\left(f_{\beta}\right) \\
p_{2}^{\alpha \beta b !}\left(\mathcal{F}_{\beta}^{\prime}\right) \stackrel{\vartheta_{\alpha \beta}^{\alpha \beta b !}\left(f_{\alpha}\right)}{\sim} p_{1}^{\alpha \beta b !}\left(\mathcal{F}_{\alpha}^{\prime}\right) .
\end{gathered}
$$

The category $F$-Isoc $\left(\left(\mathcal{X}_{\alpha}^{b}\right)_{\alpha \in \Lambda} / K\right)$ is a logarithmic analogue of the category $F$-Isoc ${ }^{\dagger \dagger}\left(\left(\mathcal{X}_{\alpha}\right)_{\alpha \in \Lambda}, Z / K\right)$ constructed in [Car09c] which we recall below for the reader :

Definition 3.6.4. We keep notation 3.6.2. The category $F$-Isoc ${ }^{\dagger \dagger}\left(\left(\mathcal{X}_{\alpha}\right)_{\alpha \in \Lambda}, Z / K\right)$ is defined as follows:

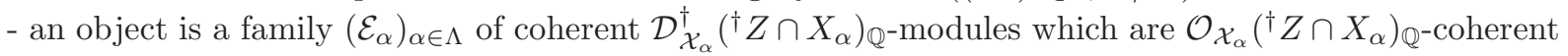
and endowed with glueing data $\left(\theta_{\alpha \beta}\right)_{\alpha, \beta \in \Lambda}$, i.e. the data for any $\alpha, \beta \in \Lambda$ of a $\mathcal{D}_{\mathcal{X}_{\alpha \beta}}^{\dagger}\left({ }^{\dagger} Z \cap X_{\alpha \beta}\right)_{\mathbb{Q}}$-linear isomorphism $\theta_{\alpha \beta}: p_{2}^{\alpha \beta !}\left(\mathcal{E}_{\beta}\right) \stackrel{\sim}{\rightarrow} p_{1}^{\alpha \beta !}\left(\mathcal{E}_{\alpha}\right)$, satisfying the cocycle condition: $\theta_{13}^{\alpha \beta \gamma}=\theta_{12}^{\alpha \beta \gamma} \circ \theta_{23}^{\alpha \beta \gamma}$, where $\theta_{12}^{\alpha \beta \gamma}$, $\theta_{23}^{\alpha \beta \gamma}$ and $\theta_{13}^{\alpha \beta \gamma}$ are defined via the commutative squares

$$
\begin{aligned}
& p_{12}^{\alpha \beta \gamma !} p_{2}^{\alpha \beta !}\left(\mathcal{E}_{\beta}\right) \stackrel{\tau}{\sim} p_{2}^{\alpha \beta \gamma !}\left(\mathcal{E}_{\beta}\right) p_{23}^{\alpha \beta \gamma !} p_{2}^{\beta \gamma !}\left(\mathcal{E}_{\gamma}\right) \stackrel{\tau}{\sim} p_{3}^{\alpha \beta \gamma !}\left(\mathcal{E}_{\gamma}\right) \quad p_{13}^{\alpha \beta \gamma !} p_{2}^{\alpha \gamma !}\left(\mathcal{E}_{\gamma}\right) \stackrel{\tau}{\sim} p_{3}^{\alpha \beta \gamma !}\left(\mathcal{E}_{\gamma}\right) \\
& \sim \downarrow_{12}^{\alpha \beta \gamma !}\left(\theta_{\alpha \beta}\right) \quad \dot{\forall} \theta_{12}^{\alpha \beta \gamma} \quad \sim \downarrow_{23}^{\alpha \beta \gamma !}\left(\theta_{\beta \gamma}\right) \quad \dot{\forall} \theta_{23}^{\alpha \beta \gamma} \quad \sim p_{13}^{\alpha \beta \gamma !}\left(\theta_{\alpha \gamma}\right) \quad \dot{\forall} \theta_{13}^{\alpha \beta \gamma} \\
& p_{12}^{\alpha \beta \gamma !} p_{1}^{\alpha \beta !}\left(\mathcal{E}_{\alpha}\right) \stackrel{\tau}{\sim} p_{1}^{\alpha \beta \gamma !}\left(\mathcal{E}_{\alpha}\right), p_{23}^{\alpha \beta \gamma !} p_{1}^{\beta \gamma !}\left(\mathcal{E}_{\beta}\right) \stackrel{\tau}{\sim} p_{2}^{\alpha \beta \gamma !}\left(\mathcal{E}_{\beta}\right), p_{13}^{\alpha \beta \gamma !} p_{1}^{\alpha \gamma !}\left(\mathcal{E}_{\alpha}\right) \stackrel{\tau}{\sim} p_{1}^{\alpha \beta \gamma !}\left(\mathcal{E}_{\alpha}\right),
\end{aligned}
$$

where the glueing isomorphisms denoted by $\tau$ are defined as in [Ber00, 2.1.5].

- a morphism $\left(\left(\mathcal{E}_{\alpha}\right)_{\alpha \in \Lambda},\left(\theta_{\alpha \beta}\right)_{\alpha, \beta \in \Lambda}\right) \rightarrow\left(\left(\mathcal{E}_{\alpha}^{\prime}\right)_{\alpha \in \Lambda},\left(\theta_{\alpha \beta}^{\prime}\right)_{\alpha, \beta \in \Lambda}\right)$ is a familly of morphisms $f_{\alpha}: \mathcal{E}_{\alpha} \rightarrow \mathcal{E}_{\alpha}^{\prime}$ commuting with glueing datas.

3.6.5 (Unipotent overcoherent $F$-isocrystal). We keep notation 3.6.2. The category $F$-Isoc ${ }^{\dagger \dagger}(Y, \mathcal{P} / K)$ is canonically isomorphic to $F$-Isoc ${ }^{\dagger \dagger}\left(\left(\mathcal{X}_{\alpha}\right)_{\alpha \in \Lambda}, Z / K\right)$ (see [Car09c]). Since in this subsection the choices are fixed, by abuse of notation we will simply denote $F-\operatorname{Isoc}\left(\left(\mathcal{X}_{\alpha}^{b}\right)_{\alpha \in \Lambda} / K\right)$ by $F-\operatorname{Isoc}\left(X^{b} / K\right)$.

From [Ked07, 6.4.5], the functor $\left({ }^{\dagger} X_{\alpha} \cap Z\right): F$-Isoc $\left(\mathcal{X}_{\alpha}^{b} / K\right) \rightarrow F$-Isoc ${ }^{\dagger \dagger}\left(\mathcal{X}_{\alpha}, Z \cap \mathcal{X}_{\alpha} / K\right)$ is fully faithful. Hence, we get the fully faithful functor $F$-Isoc $\left(X^{b} / K\right) \rightarrow F$-Isoc ${ }^{\dagger \dagger}\left(\left(\mathcal{X}_{\alpha}\right)_{\alpha \in \Lambda}, Z / K\right)$ which is defined by sending $\left(\left(\mathcal{F}_{\alpha}\right)_{\alpha \in \Lambda},\left(\vartheta_{\alpha \beta}\right)_{\alpha, \beta \in \Lambda}\right)$ to $\left(\left(\mathcal{F}_{\alpha}\left({ }^{\dagger} X_{\alpha} \cap Z\right)_{\alpha \in \Lambda},\left(\theta_{\alpha \beta}\right)_{\alpha, \beta \in \Lambda}\right)\right.$ where $\theta_{\alpha \beta}$ are the isomorphisms induced 
canonically by $\vartheta_{\alpha \beta}$. By composing this functor with the glueing equivalence $F$-Isoc ${ }^{\dagger \dagger}\left(\left(\mathcal{X}_{\alpha}\right)_{\alpha \in \Lambda}, Z / K\right) \cong$ $F$-Isoc ${ }^{\dagger \dagger}(Y, \mathcal{P} / K)$, we get a fully faithful functor which will be denoted by

$$
\left({ }^{\dagger} Z\right): F-\operatorname{Isoc}\left(X^{b} / K\right) \rightarrow F-\operatorname{Isoc}^{\dagger \dagger}(Y, \mathcal{P} / K) .
$$

To justify this notation, notice that when $\mathcal{P}_{\alpha}=\mathcal{P}$ for any $\alpha$ then the functor (3.6.5.1) is equal to the usual functor $\left({ }^{\dagger} Z\right)$ as in the lifted case 3.1.5. The essencial image of this functor $\left({ }^{\dagger} Z\right)$ are by definition the unipotent (overcoherent) $F$-isocrystals.

3.6.6 (Residue morphism, monodromy filtration). We keep the notation 3.6.5. By gluing (3.2.10.1), we get the factorisation

$$
i_{1}^{*}: F-\operatorname{Isoc}\left(X^{b} / K\right) \rightarrow F-\operatorname{Isoc}\left(Z_{1}^{\#} / K\right),
$$

where $i_{1}: Z_{1} \hookrightarrow X$ is the closed immersion and $F$-Isoc $\left(Z_{1}^{\#} / K\right)$ is constructed similarly.

Let $\mathcal{F} \in F$-Isoc $\left(X^{\mathrm{b}} / K\right)$ and $\mathcal{H}:=i_{1}^{*}(\mathcal{F})$. We note that in the situation of $\S 3.1$, the nilpotent homomorphism $N_{1, \mathcal{F}}$ induced by the action of $t_{1} \partial_{1}$ on $\mathcal{H}$ does not depend on the choice of local coordinates $t_{1}, \ldots, t_{d}$ such that $Z_{1}=V\left(t_{1}\right)$. Thus, by gluing, we may construct as in 3.2.11 the nilpotent homomorphism $N_{1, \mathcal{F}}: \mathcal{H}(1) \rightarrow \mathcal{H}$ in $F$-Isoc $\left(Z_{1}^{\#} / K\right)$, the residue morphism of $\mathcal{F}$ corresponding to $Z_{1}$. As in 3.2.6, we check that there exists a unique finite increasing filtration $M$ on $\mathcal{H}$ such that $N_{1, \mathcal{F}} M_{i} \subset M_{i-2}(-1)$, and $N_{1, \mathcal{F}}^{k}$ induces an isomorphism $\operatorname{gr}_{k}^{M}(\mathcal{H}) \stackrel{\sim}{\rightarrow} \operatorname{gr}_{-k}^{M}(\mathcal{H})(-k)$. We call this filtration the monodromy filtration of $\left(\mathcal{H}, N_{1, \mathcal{F}}\right)$.

3.6.7. We define the category $\operatorname{ULNM}(Y, \mathcal{P} / K)$ to be the full subcategory of $F$-Isoc ${ }^{\dagger \dagger}(Y, \mathcal{P} / K)$ consisting of $\mathcal{E}$ such that: 1 . $\mathcal{E}$ is unipotent, and $2 . \mathcal{E}$ is the restriction of an $\iota$-mixed overconvergent $F$-isocrystal on $Y$.

Theorem 3.6.8. Let $j=(\star, \mathrm{id}, \mathrm{id}):(Y, X, \mathcal{P}) \rightarrow(X, X, \mathcal{P})$ be the morphism of frames.

(i) Suppose $X$ proper. For any $\mathcal{E} \in \operatorname{ULNM}(Y, \mathcal{P} / K)$, then $j_{!}(\mathcal{E}) \|_{W}$ is an ı-mixed $F$-isocrystal for any stratum $W$ of the stratification $\operatorname{Strat}_{Z}(X)$. In particular, $j_{!}(\mathcal{E})$ is an $\iota$-mixed $F$-complex.

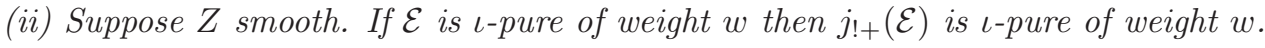

Proof. Using [Ked07, 3.2.20], we can assume by dévissage that $\mathcal{E}$ is $\iota$-pure of weight $w$. Since $\mathcal{E}$ is a unipotent $F$-isocrystal, there exists a (unique up to isomorphism) an object $\mathcal{F}$ of $F-\operatorname{Isoc}\left(X^{b} / K\right) \operatorname{such}$ that $\mathcal{E} \stackrel{\sim}{\rightarrow}\left({ }^{\dagger} Z\right)(\mathcal{F})$ (see (3.6.5.1)).

Let us start to show (i). We proceed by induction on $r$. For $r=0$, we have $\mathcal{Z}=\emptyset$ and this case is obvious. Suppose now that $r \geq 1$ and the proposition is checked for $r^{\prime}<r$.

a) Let $D_{1}:=Z_{2} \cup \cdots \cup Z_{r}, Y_{1}:=Z_{1} \backslash D_{1}$, and $j_{1}: Y_{1} \rightarrow Z_{1}$ be the open immersion. From 3.6.6, we get an element $\mathcal{H}:=i_{1}^{*}(\mathcal{F})$ of $F-\operatorname{Isoc}\left(Z_{1}^{\#} / K\right)$ and a nilpotent homomorphism $N_{1, \mathcal{F}}: \mathcal{H}(1) \rightarrow \mathcal{H}$ in $F$-Isoc $\left(Z_{1}^{\#} / K\right)$. Hence, we get the objects $\mathcal{F}_{1}:=\operatorname{ker} N_{1, \mathcal{F}}$ and $\mathcal{F}_{2}:=\operatorname{coker} N_{1, \mathcal{F}}$ of $F$-Isoc $\left(Z_{1}^{\#} / K\right)$. The monodromy filtration on $\mathcal{H}$ induces canonically some filtration on $\mathcal{F}_{1}$ and $\mathcal{F}_{2}$. Since the functor $\left({ }^{\dagger} D_{1}\right): F-\operatorname{Isoc}\left(Z_{1}^{\mathrm{b}} / K\right) \rightarrow F-\operatorname{Isoc}^{\dagger \dagger}\left(Y_{1}, \mathcal{P} / K\right)$ (defined as in (3.6.5.1)) is exact, the filtrations on $\mathcal{F}_{1}$ and $\mathcal{F}_{2}$ induce canonically a filtration on the unipotent $F$-isocrystals $\mathcal{E}_{1}:=\left({ }^{\dagger} D_{1}\right)\left(\mathcal{F}_{1}\right)$ and $\mathcal{E}_{2}:=\left({ }^{\dagger} D_{1}\right)\left(\mathcal{F}_{2}\right)$ of $F$-Isoc ${ }^{\dagger \dagger}\left(Y_{1}, \mathcal{P} / K\right)$, which will be denoted by $M$. Then $\operatorname{gr}_{i}^{M}\left(\mathcal{E}_{1}\right), \operatorname{gr}_{i}^{M}\left(\mathcal{E}_{2}\right)$ are $\iota$-pure. Indeed, by Remark 2.1.10, the verification is local, and we may reduce to the situation of Theorem 3.3.5. Then by this theorem the purity follows. Hence, by induction hypothesis, $j_{1 !}\left(\mathcal{E}_{1}\right) \|_{W}$ and $j_{1 !}\left(\mathcal{E}_{2}\right) \|_{W}$ are in $D_{\text {isoc,m }}^{\mathrm{b}}(W, \mathcal{P} / K)$ for any stratum $W$ of $\operatorname{Strat}_{D_{1}}\left(Z_{1}\right)$.

b) On the other hand, for any stratum $W$ of $\operatorname{Strat}_{D_{1}}\left(Z_{1}\right)$, we have isomorphisms for any $k \in \mathbb{Z}$

$$
\mathcal{H}_{\mathrm{t}}^{k}\left(\mathcal{H}_{Z_{1}}^{\dagger 0}\left(j_{!}(\mathcal{E})\right) \|_{W}\right) \cong \mathcal{H}_{\mathrm{t}}^{k}\left(j_{1 !}\left(\mathcal{E}_{1}\right) \|_{W}\right), \quad \mathcal{H}_{\mathrm{t}}^{k}\left(\mathcal{H}_{Z_{1}}^{\dagger 1}\left(j_{!}(\mathcal{E})\right) \|_{W}\right) \cong \mathcal{H}_{\mathrm{t}}^{k}\left(j_{1 !}\left(\mathcal{E}_{2}\right) \|_{W}\right) .
$$

Indeed, first notice that the right sides of both isomorphisms are objects of $F$-Isoc ${ }^{\dagger \dagger}(W, \mathcal{P} / K)$. Moreover, the fact that an object of $F$-Ovhol $(W, \mathcal{P} / K)$ is an object of $F$-Isoc ${ }^{\dagger \dagger}(W, \mathcal{P} / K)$ is local in $\mathcal{P}$. Hence, by using Kedlaya's fully faithfulness theorems [Ked07, 5.2.1] (in fact we only need [Tsu02, 4.1.1] since $Y$ is smooth) and [Ked04], the check of the isomorphisms (3.6.8.1) is local. Hence, we may assume that we are in the situation of $\S 3.1$. Thus, by Theorem 3.5.13, the isomorphisms follows. 
c) Combining a) and b), we get that $\mathcal{H}_{Z_{1}}^{\dagger k}\left(j_{!}(\mathcal{E})\right) \|_{W}(k=0,1)$ is in $D_{\text {isoc,m }}^{\mathrm{b}}(W, \mathcal{P} / K)$. Since we have the triangle

$$
\mathcal{H}_{Z_{1}}^{\dagger 0}\left(j_{!}(\mathcal{E})\right)\left\|_{W} \rightarrow \mathbb{R} \underline{\Gamma}_{Z_{1}}^{\dagger}\left(j_{!}(\mathcal{E})\right)\right\|_{W} \rightarrow \mathcal{H}_{Z_{1}}^{\dagger 1}\left(j_{!}(\mathcal{E})\right) \|_{W} \stackrel{+}{\rightarrow}
$$

we conclude by Lemma 2.1 .12 that $j_{!}(\mathcal{E})\left\|_{W} \cong \mathbb{R}_{Z_{1}}^{\dagger}\left(j_{!}(\mathcal{E})\right)\right\|_{W}$ is in $D_{\text {isoc, } \mathrm{m}}^{\mathrm{b}}(W, \mathcal{P} / K)$ for any stratum $W$ in $\operatorname{Strat}_{D_{1}}\left(Z_{1}\right)$.

By replacing $Z_{1}$ by $Z_{2}, \ldots, Z_{r}$ and $D_{1}$ by $D_{i}:=\bigcup_{j \neq i} Z_{j}$, we get the same result. By construction, any stratum of $\operatorname{Strat}_{Z}(X)$ except for $Z_{(0)}^{\circ}=Y$ is a disjoint union of strata in $\operatorname{Strat}_{D_{i}}\left(Z_{i}\right)$. Thus the theorem follows.

Let us show (ii). Since the verification is local, we may assume that we are in the situation of $\S 3.1$ and since $Z$ is smooth we can suppose $\mathcal{Z}=\mathcal{Z}_{1}$. Consider the exact sequence:

$$
0 \rightarrow j_{!+}(\mathcal{E}) \rightarrow j_{+}(\mathcal{E}) \rightarrow \mathcal{H}_{Z}^{\dagger 1}\left(j_{!}(\mathcal{E})\right) \rightarrow 0
$$

From 3.5.13, we get the isomorphism $\mathcal{H}_{Z}^{\dagger 1}\left(j_{!}(\mathcal{E})\right) \stackrel{\sim}{\longrightarrow} i_{1+}\left(\right.$ coker $\left.N_{1, \mathcal{F}}\right)$ compatible with Frobenius. Using the Corollory 3.3.6, this yields that $\mathcal{H}_{Z}^{\dagger 1}\left(j_{!}(\mathcal{E})\right)$ is $\iota$-mixed of weight $\geq w+1$. Usinf by Lemma 2.2.6, we get that $j_{+}(\mathcal{E})$ is $\iota$-mixed of weight $\geq w$. This implies by Proposition 2.2.7.1 (use also the above exact sequence) that $j_{!+}(\mathcal{E})$ is $\iota$-mixed of weight $\geq w$. By Corollary 1.4.3 and Lemma 2.1.6.3, we get that $j_{!++}(\mathcal{E})$ is $\iota$-mixed of weight $\leq w$.

Remark 3.6.9. The second condition in the definition of $\operatorname{ULNM}(Y, \mathcal{P} / K)$ is used to apply Theorem 3.3.5. If we remove this condition, we do not know if Theorem 3.6.8 holds or not.

\section{Theory of weights}

Throughout this section, we consider situation (B) in Notation and convention.

\subsection{Stability of mixedness}

4.1.1. Let $\star \in\{\emptyset, \leq w, \geq w\}$. Let $Y$ be a realizable variety and $\mathcal{E} \in F$ - $D_{\text {ovhol }}^{\mathrm{b}}(Y / K)$. We say that $\mathcal{E}$ satisfies the condition $(\mathrm{SQ})_{\star}$ if the following holds:

$(\mathrm{SQ})_{\star}$ For any integer $i$, any subquotient of $\mathcal{H}_{\mathrm{t}}^{i}(\mathcal{E})$ is $\iota$-mixed of weight $\star+i$.

Theorem 4.1.2. Let $f: X \rightarrow Y$ be a morphism of realizable varieties.

(i) For any $\mathcal{E} \in F-D_{\geq w}^{\mathrm{b}}(X / K)$, we have $f_{+}(\mathcal{E}) \in F-D_{\geq w}^{\mathrm{b}}(Y / K)$.

(ii) The dual functor $\mathbb{D}_{X}$ exchanges $F-D_{\geq w}^{\mathrm{b}}(X / K)$ and $F-D_{\leq-w}^{\mathrm{b}}(X / K)$.

(iii) The condition (SQ) holds for any $F$-complex in $F-D_{\mathrm{m}}^{\mathrm{b}}(\bar{X} / K)$.

Remark. See Theorem 4.2.3 for more complete results for (iii).

Proof. 0) Preliminaries.

a) Dévissage in $\mathcal{E}$ : Let $\mathcal{E}^{\prime} \rightarrow \mathcal{E} \rightarrow \mathcal{E}^{\prime \prime} \stackrel{+}{\rightarrow}$ be an exact triangle of $F-D_{\geq w}^{\mathrm{b}}(X / K)$. By Lemma 2.2 .7 , if the part (i) and (ii) of the theorem holds for $\mathcal{E}^{\prime}$ and $\mathcal{E}^{\prime \prime}$, then so does for $\mathcal{E}$.

b) Dévissage in $X$ : Let $j: U \hookrightarrow X$ be an open immersion and $i: X \backslash U \hookrightarrow X$ be the complement. Then if the theorem holds for $f \circ j$ and $f \circ i$ then so does for $f$. This follows from Lemma 2.2.7.

c) Dévissage in $Y$ : Let $V$ be a open subvariety of $Y$ and $W:=Y \backslash V$. If the theorem holds for $f^{\prime}: f^{-1}(V) \rightarrow V$ and $f^{\prime \prime}: f^{-1}(W) \rightarrow W$ then so does for $f$. Indeed, by Lemma 2.2.6, the theorem holds for the morphisms $f^{-1}(V) \stackrel{f^{\prime}}{\rightarrow} V \hookrightarrow Y$ and $f^{-1}(W) \stackrel{f^{\prime \prime}}{\longrightarrow} W \hookrightarrow Y$, and by b) the claim follows.

d) Let $\mathcal{E}^{\prime} \rightarrow \mathcal{E} \rightarrow \mathcal{E}^{\prime \prime} \stackrel{+}{\rightarrow}$ be a triangle, and assume that (SQ) holds for $\mathcal{E}^{\prime}$ and $\mathcal{E}^{\prime \prime}$. Then (SQ) holds for $\mathcal{E}$ as well. 
1) By Lemma 2.2.6, the theorem holds when $X$ is of dimension 0 and we may assume that $\operatorname{dim}(Y) \leq \operatorname{dim}(X)$. We proceed by induction on the dimension of $X$. Assume the theorem holds for $\operatorname{dim}(X)<n$. We will show the theorem for $\operatorname{dim}(X)=n$. Let $\mathcal{E} \in F-D_{\geq w}^{\mathrm{b}}(X / K)$.

2) a) Let us show (i) in the case where $f$ is quasi-finite. If $f$ is an immersion, then by Lemma 2.2.6 the theorem holds. Hence, by using EGA IV, Theorem 8.12.6, we may assume that $f$ is finite. By using 0.a) and 0.b), we may assume that $X$ is smooth, $\mathcal{E} \in F$-Isoc ${ }^{\dagger \dagger}(X / K)$ and it is $\iota$-pure of weight $w$. Moreover, thanks to 0.c) and the induction hypothesis, we may further suppose that $Y$ is smooth and that $f_{+}(\mathcal{E}) \in F-D_{\text {isoc }}^{\mathrm{b}}(Y / K)$. Then it is sufficient to check that for every closed point $y$ of $Y, i_{y}^{!} \circ f_{+}(\mathcal{E})$ is $\iota$-pure of weight $w$ where $i_{y}:\{y\} \hookrightarrow Y$ is the closed immersion as usual. Hence, by using a base change theorem 1.3.14, we reduce to the case where $\operatorname{dim} X=\operatorname{dim} Y=0$, and the theorem follows.

b) Assume $f$ is a universal homeomorphism. From 2.a) and Proposition 2.2.4, $f_{+}$and $f^{!}$preserve the mixedness $\geq w$. Thus, by Proposition 1.3.12, the functors $f_{+}$and $f^{!}$induce canonical equivalences of categories between $F-D_{\geq w}^{\mathrm{b}}(X / K)$ and $F-D_{\geq w}^{\mathrm{b}}(Y / K)$.

3) Let us show (ii) and (iii).

a) Let us show (ii) for $\operatorname{dim}(X)=n$. We may assume $X$ to be proper. By dévissage, it suffices to show that for an open affine smooth subscheme $j: U \hookrightarrow X$ and an $\iota$-mixed overconvergent $F$-isocrystal $\mathcal{E}$ on $U, j_{!}(\mathcal{E})$ is $\iota$-mixed. By using Kedlaya's semistable reduction theorem [Ked11], there exists a generically finite étale morphism $g: X^{\prime} \rightarrow X$ such that $X^{\prime}$ is projective smooth, $Z^{\prime}:=X^{\prime} \backslash g^{-1}(U)$ is a strictly normal crossing divisor, and $g_{U}^{*}(\mathcal{E})$ is a unipotent $F$-isocrystal where $g_{U}: g^{-1}(U) \rightarrow U$. There exists an open subscheme $V \subset U$ such that $g_{V}: V^{\prime}:=g^{-1}(V) \rightarrow V$ is finite étale. For $\star \in\{U, V\}$, let $j_{\star}: \star \hookrightarrow X, j_{\star}^{\prime}: g^{-1}(\star) \hookrightarrow X^{\prime}$. Now, for a $\iota$-mixed $F$-module $\mathcal{F}$ on $X^{\prime}$, we claim that $g_{+}(\mathcal{F})$ is $\iota$-mixed. Indeed, consider the triangle $\mathbb{R} \Gamma_{X^{\prime} \backslash V^{\prime}}^{\dagger}(\mathcal{F}) \rightarrow \mathcal{F} \rightarrow j_{V+}^{\prime} \mathcal{F} \|_{V^{\prime}} \stackrel{+}{\rightarrow}$. By induction hypothesis, $g_{+}\left(\mathbb{R} \Gamma_{X^{\prime} \backslash V^{\prime}}^{\dagger}(\mathcal{F})\right)$ is $\iota$-mixed, and since $g_{V}$ is finite étale, by 2.a), $g_{+} j_{V+}^{\prime} \mathcal{F} \|_{V^{\prime}}$ is $\iota$-mixed as well, and the claim follows.

By Theorem 3.6.8, $j_{U !}^{\prime}\left(g_{U}^{*} \mathcal{E}\right)$ is $\iota$-mixed. By using the induction hypothesis, this is equivalent to the property that $j_{V !}^{\prime}\left(g_{U}^{*}(\mathcal{E}) \| V^{\prime}\right)$ is $\iota$-mixed as well. Now, by the claim, so is $g_{+} j_{V !}^{\prime}\left(g_{U}^{*}(\mathcal{E}) \| V^{\prime}\right)$. Since it contains $j_{V !}(\mathcal{E} \| V)$ as a direct factor, then $j_{V !}(\mathcal{E} \| V)$ is $\iota$-mixed, and using the induction hypothesis again, we get that $j_{!}(\mathcal{E})$ is $\iota$-mixed.

b) Let us show (iii) for $\operatorname{dim}(X)=n$. For $\mathcal{E}$, there exists an open subscheme $j$ : $U \hookrightarrow X$ such that $U$ is smooth and the complement of a divisor in $X$ and $\mathcal{E} \|_{U}$ is an isocrystal. By considering the localization triangle, 0.d), and induction hypothesis, it suffices to show (SQ) for $j_{+}(\mathcal{F})$ where $\mathcal{F}$ is an irreducible overconvergent $F$-isocrystal on $U$. Then, by (1.4.4.1), we get the exact sequence

$$
0 \rightarrow j_{!_{+}}(\mathcal{F}) \rightarrow j_{+}(\mathcal{F}) \rightarrow \mathbb{R}^{1} \underline{\Gamma}_{Z}^{\dagger}\left(j_{!}(\mathcal{F})\right) \rightarrow 0 .
$$

We have already shown at 2.a) that $j_{+}(\mathcal{F})$ and $j_{!}(\mathcal{F})$ are $\iota$-mixed. Thus, $\mathbb{R} \underline{\Gamma}_{Z}^{\dagger}\left(j_{!}(\mathcal{F})\right)$ is $\iota$-mixed, and $\mathbb{R}^{1} \underline{\Gamma}_{Z}^{\dagger}\left(j_{!}(\mathcal{F})\right)$ is $\iota$-mixed by the induction hypothesis on (SQ). Thus, by $\left.0 . \mathrm{d}\right), j_{!_{+}}(\mathcal{F})$ is $\iota$-mixed. Since $j_{!_{+}}(\mathcal{F})$ is irreducible by Proposition 1.4.7, this satisfies (SQ). Thus, (SQ) for $j_{+}(\mathcal{F})$ follows by $0 . \mathrm{d}$ ).

4) It remains to check (i). By using 0), 2.a), and Noether's normalization theorem, we may assume that $f$ is a smooth morphism of relative dimension $1, X$ is integral smooth, and $\mathcal{E} \in F$-Isoc ${ }^{\dagger \dagger}(X / K)$. By 0.c), we may assume that $Y$ is integral, affine and smooth. We choose $h: \bar{X} \rightarrow Y$ a proper morphism of realizable varieties, an open immersion $j: X \hookrightarrow \bar{X}$ such that $f=h \circ j$ and $X$ is dense in $\bar{X}$. By using Kedlaya's semistable reduction theorem, there exist a projective surjective morphism $g: \bar{X}^{\prime} \rightarrow \bar{X}$ such that the induced morphism $g^{\prime}: X^{\prime}:=g^{-1}(X) \rightarrow X$ is generically finite étale, $\bar{X}^{\prime}$ is quasi-projective, integral, smooth, $D^{\prime}:=\bar{X}^{\prime} \backslash X^{\prime}$ is a strict normal crossing divisor of $\bar{X}^{\prime}$ and $g^{\prime *}(\mathcal{E})$ is a unipotent along $D^{\prime}$ overconvergent $F$-isocrystal. Let $U$ be an open dense subvariety of $X$ such that the induced morphism $U^{\prime}:=g^{-1}(U) \rightarrow U$ is finite étale. Let $\mathcal{K}$ be the function field of $Y$. Let $\bar{X}_{\mathcal{K}}^{\prime}$, and $U_{\mathcal{K}}^{\prime}$ be the fibers of the generic element of $Y$. Since $U_{\mathcal{K}}^{\prime}$ is a smooth curve over $\operatorname{Spec}(\mathcal{K})$, we have its canonical smooth compactification $C_{\mathcal{K}}^{\prime}$ over $\mathcal{K}$. Since $\bar{X}_{\mathcal{K}}^{\prime}$ is projective over $\mathcal{K}$, there exists a unique finite morphism $\alpha_{\mathcal{K}}: C_{\mathcal{K}}^{\prime} \rightarrow \bar{X}_{\mathcal{K}}^{\prime}$, which is nothing but the normalization morphism, 
that extends the inclusion $U_{\mathcal{K}}^{\prime} \rightarrow \bar{X}_{\mathcal{K}}^{\prime}$. By using EGA IV, Theorem 8.8.2 and shrinking $Y$, we may assume that $C_{\mathcal{K}}^{\prime}$ comes from a variety $C^{\prime}$ which is projective smooth of relative dimension 1 over $Y$. By using EGA IV, Theorems 8.8.2, 8.10.5, 17.7.8, by shrinking $Y$ further, we can assume that $\alpha_{\mathcal{K}}$ (resp. the open immersion $U_{\mathcal{K}}^{\prime} \hookrightarrow C_{\mathcal{K}}^{\prime}$ ) comes from a finite morphism $\alpha: C^{\prime} \rightarrow \bar{X}^{\prime}$ (resp. an open immersion $j^{\prime}: U^{\prime} \hookrightarrow C^{\prime}$ ) such that $\alpha \circ j^{\prime}$ is the canonical open immersion. Since $\alpha$ is a finite morphism of smooth integral varieties and since $\alpha \circ j^{\prime}$ is an open immersion then $\alpha$ is an isomorphism. Since $\mathcal{E}$ is a direct factor of $g_{+}^{\prime} g^{\prime *}(\mathcal{E})$ (because $g$ is generically finite and etale and $\mathcal{E}$ is an isocrystal), then $f_{+}(\mathcal{E})$ is a direct factor of $\left(f \circ g^{\prime}\right)_{+} g^{\prime *}(\mathcal{E})$. Hence, we can suppose that there exists the following commutative diagram of varieties

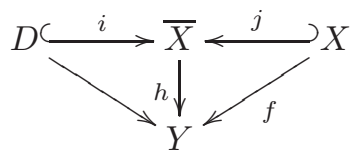

where $h$ is projective smooth purely of relative dimension $1, j$ is an open immersion, $D:=\bar{X} \backslash X$ is a strict normal crossing divisor of $\bar{X}$ with $i$ the induced closed immersion, $\mathcal{E}$ is $\iota$-pure of weight $w$ having unipotent monodromy along $D$. Now, there exists a finite radicial extension $\mathcal{L}$ of $\mathcal{K}$ such that the morphism $D_{\mathcal{L}} \rightarrow \operatorname{Spec}(\mathcal{L})$ is finite étale. Let $a: \widetilde{Y} \rightarrow Y$ be the normalization of $Y$ in $\mathcal{L}$. By 2.b), we may take the the pull-back by $a$, and shrinking $Y$ further, we may assume moreover that $h \circ i$ is finite étale. By shrinking $Y$ once again, we may assume $h_{+}\left(j_{!_{+}}(\mathcal{E})\right) \in F-D_{\text {isoc }}^{\mathrm{b}}(Y / K)$.

5) Let us finish the proof of (i). By 3.a), we know that $j_{!}(\mathcal{E})$ is $\iota$-mixed. Thus, $\mathbb{R} \underline{\Gamma}_{D}^{\dagger}\left(j_{!}(\mathcal{E})\right)$ is $\iota$-mixed as well, and since (SQ) holds for this $F$-complex, we get that $\mathbb{R}^{1} \underline{\Gamma}_{D}^{\dagger}\left(j_{!}(\mathcal{E})\right)$ is $\iota$-mixed. Thus, we get that $j_{!_{+}}(\mathcal{E})$ is $\iota$-mixed. Moreover, by Theorem 3.6.8 (ii), $j_{!_{+}}(\mathcal{E})$ is $\iota$-pure of weight $w$. Now, let us show that $h_{+}\left(j_{!_{+}}(\mathcal{E})\right)$ is $\iota$-pure of weight $w$. For this, it suffices to show that for an $\iota$-pure $F$-module $\mathcal{F}$ of weight $w$ on $\bar{X}$ such that $h_{+}(\mathcal{F}) \in F$ - $D_{\text {isoc }}^{\mathrm{b}}(Y / K), h_{+}(\mathcal{F})$ is $\iota$-pure of weight $w$. Let $y$ be a closed point of $Y$, and $i_{y}:\{y\} \hookrightarrow Y$ be the closed immersion. We put $i_{y}^{\prime}: h^{-1}(y) \hookrightarrow \bar{X}$ the closed immersion, and $h_{y}^{\prime}: h^{-1}(y) \rightarrow\{y\}$ the proper smooth curve. By base change theorem 1.3.14, we have $i_{y}^{!} h_{+}(\mathcal{F}) \cong h_{y+}^{\prime} i_{y}^{\prime \prime}(\mathcal{F})$. Since $i_{y}^{\prime !}(\mathcal{F})$ is $\iota$-mixed of weight $\geq w$, by Proposition 2.2.4, $i_{y}^{!} h_{+}(\mathcal{F})$ is $\iota$-mixed of weight $\geq w$. Since $h$ is proper, $h_{!} \cong h_{+}$by paragraph 1.3.14. By 3.a), since $\mathbb{D}_{\bar{X}}(\mathcal{F})$ is $\iota$-pure of weight $-w$, we get that $i_{y}^{+} h_{+}(\mathcal{F})$ is $\iota$-mixed of weight $\leq w$, and we conclude that $h_{+}(\mathcal{F})$ is $\iota$-pure of weight $w$.

Finally, consider the exact sequence

$$
0 \rightarrow j_{!+} \mathcal{E} \rightarrow j_{+} \mathcal{E} \rightarrow \mathcal{H}_{\mathrm{t}}^{1} \mathbb{R} \underline{\Gamma}_{D}^{\dagger}\left(j_{!}(\mathcal{E})\right) \rightarrow 0 .
$$

Since $j_{!+}(\mathcal{E})$ and $j_{+}(\mathcal{E})$ are $\iota$-mixed of weight $\geq w$, so is $\mathcal{H}_{\mathrm{t}}^{1} \mathbb{R} \underline{\Gamma}_{D}^{\dagger}\left(j_{!}(\mathcal{E})\right)$ by Lemma 2.2.7. By the finite étaleness of $h \circ i, h_{+}\left(\mathcal{H}_{\mathrm{t}}^{1} \mathbb{R} \Gamma_{D}^{\dagger}(j !(\mathcal{E}))\right)$ is $\iota$-mixed of weight $\geq w$. Since we showed that $h_{+}\left(j_{!+}(\mathcal{E})\right)$ is $\iota$-pure of weight $w$, we get that $f_{+}(\mathcal{E}) \cong h_{+}\left(j_{+}(\mathcal{E})\right)$ is $\iota$-mixed of weight $\geq w$, which concludes the proof.

4.1.3. Let us sum up the results.

Theorem. Let $f: X \rightarrow Y$ be a morphism of realizable varieties. We get:

1. $f$ ! and $f^{+}$preserve $D_{\leq w}^{\mathrm{b}}$;

2. $f^{!}$and $f_{+}$preserve $D_{\geq w}^{\mathrm{b}}$;

3. $\widetilde{\otimes}_{Y}$ sends $D_{\geq w}^{\mathrm{b}} \times D_{\geq w^{\prime}}^{\mathrm{b}}$ to $D_{\geq w+w^{\prime}}^{\mathrm{b}}$, and $\otimes_{Y}$ sends $D_{\leq w}^{\mathrm{b}} \times D_{\leq w^{\prime}}^{\mathrm{b}}$ to $D_{\leq w+w^{\prime}}^{\mathrm{b}}$;

4. $\mathbb{D}_{Y}$ exchanges $D_{\leq w}^{\mathrm{b}}$ and $D_{\geq-w}^{\mathrm{b}}$. 


\subsection{Purity results}

In this subsection, we prove the purity stability for intermediate extension. With this purity, we complete the theory of weights in $p$-adic cohomology theory. For the proof of Theorem 4.2.2, we follow the idea of Kiehl-Weissauer [KW01].

Lemma 4.2.1. Let $X$ be a realizable variety, and $\left\{X_{t}\right\}_{t \in I}$ be a set of divisors of $X$ such that $X_{t} \cap X_{t^{\prime}}=\emptyset$ for any $t \neq t^{\prime}$ in $I$. Let $i_{t}: X_{t} \hookrightarrow X$ be the closed immersion. Then for any overholonomic $F$-module $\mathcal{E}$ over $X$, the $F$-complex $i_{t}^{!} \mathcal{E}[1]$ is in fact a $F$-module (or equivalently $\mathcal{H}_{\mathrm{t}}^{0} i_{t}^{!} \mathcal{E}=0$ ) for any but finitely many $t \in I$.

Proof. We have an injection $i_{t+} \mathcal{H}_{\mathrm{t}}^{0}\left(i_{t}^{!} \mathcal{E}\right) \hookrightarrow \mathcal{E}$. Since $i_{t+} \mathcal{H}_{\mathrm{t}}^{0}\left(i_{t}^{!} \mathcal{E}\right)$ is supported on $X_{t}$, different $t \in I$ give different $F$-submodules of $\mathcal{E}$. Since there are finitely many constituents of an overholonomic $F$-module, we conclude that $\mathcal{H}_{\mathrm{t}}^{0}\left(i_{t}^{1} \mathcal{E}\right)=0$ except for finitely many $t$ in $I$. In this case, since $X_{t}$ is assumed to be a divisor, $i_{t}^{!} \mathcal{E}$ is concentrated at degree 1 , and the lemma follows.

Theorem 4.2.2. Let $X$ be a realizable variety, and $i: Z \hookrightarrow X$ be a closed subvariety whose complement is denoted by $U$. Let $\mathcal{E}$ be an overholonomic $F$-module on $X$ which is $\iota$-mixed and such that $\mathcal{H}_{\mathrm{t}}^{0} i ! \mathcal{E}=0$. If $\mathcal{E} \|_{U}$ is $\iota$-mixed of weight $\geq w$, so is $\mathcal{E}$.

Proof. Since the verification is local, we may assume that $X$ is affine. Take a closed embedding $X \hookrightarrow \mathbb{A}_{k}^{n}$ for some $n$. The assumption being stable under closed push-forward, we assume $X$ to be $\mathbb{A}_{k}^{n}$ from now on. Now, we will show the theorem using the induction on $n$. Assume that the theorem holds for $n<N$.

Let us, first, reduce to the case $\operatorname{dim}(Z)=0$. Choose a linear projection $X=\mathbb{A}_{k}^{N} \rightarrow T:=\mathbb{A}_{k}^{1}$. For $t \in|T|$, we denote $i_{t}: X_{t}:=X \times_{T}\{t\} \hookrightarrow X$, the induced closed immersion. If there is no risk of confusion, we denote by $i: Z_{t}:=Z \times_{T}\{t\} \hookrightarrow X_{t}$ and $i_{t}: Z_{t}:=Z \times_{T}\{t\} \hookrightarrow Z$ the induced closed immersions. By Lemma 4.2.1, there exists a finite set $B \subset|T|$ of closed points such that, for any $t \notin B$, we have $\mathcal{H}_{\mathrm{t}}^{0} i_{t}^{!} \mathcal{E}=0$. By using Lemma 4.2.1 once again, by enlarging $B$ if necessary, we have $\mathcal{H}_{\mathrm{t}}^{0} i_{t}^{!}\left(\mathcal{H}_{\mathrm{t}}^{1} i^{!} \mathcal{E}\right)=0$ for any $t \notin B$. Since the assumption shows that $\mathcal{H}_{\mathrm{t}}^{0} i^{!} \mathcal{E}=0$, we get $\mathcal{H}_{\mathrm{t}}^{1}\left(i_{t}^{!} i^{!} \mathcal{E}\right)=0$ for any $t \notin B$. Since $i_{t}^{!} i^{!} \stackrel{\sim}{\rightarrow} i^{!} i_{t}^{!}$, this implies that $\mathcal{H}_{\mathrm{t}}^{0} i^{!} \mathcal{H}_{\mathrm{t}}^{1} i_{t}^{!} \mathcal{E}=0$ for any $t \notin B$. Since $\mathcal{E} \|_{U}$ is $\iota$-mixed of weight $\geq w$, then $\mathcal{H}_{\mathrm{t}}^{1} i_{t}^{!}(\mathcal{E}) \|_{U_{t}}$ is $\iota$-mixed of weight $\geq w+1$, where $U_{t}:=U \times_{T}\{t\}$. Hence, by induction, we get that $\mathcal{H}_{\mathrm{t}}^{1} i_{t}^{i} \mathcal{E}$ is $\iota$-mixed of weight $\geq w+1$, for any $t \notin B$. By applying the same argument for the other projection $\mathbb{A}_{k}^{N} \rightarrow \mathbb{A}_{k}^{1}$, we can check there exists a closed subscheme $Z^{\prime} \subset Z$ of dimension 0 such that $\mathcal{E}$ is $\iota$-mixed of weight $\geq w$ on $X \backslash Z^{\prime}$. By the transitivity and left exactness of $i^{\text {! }}$, we may replace $Z$ by $Z^{\prime}$.

Now, let us finish the proof by treating the case $\operatorname{dim}(Z)=0$. Since the weight do not change by extension of $K$ by Lemma 2.2.10, we may assume $\pi \in K$ to use geometric Fourier transform. We put $T_{\pi}(\mathcal{E}):=\mathcal{F}_{\pi}(\mathcal{E})[2]$ and $\mathcal{J}_{\pi}:=\mathcal{K}_{\pi}[2]$, which makes the description slightly simpler because the weight of $\mathcal{J}_{\pi}$ is 0 . Let $x \in\left|\mathbb{A}_{k}^{N}\right|$, and $k(x)$ be its residue field, $\iota_{x}: \operatorname{Spec} k(x) \hookrightarrow \mathbb{A}^{N}, \alpha_{x}: \mathbb{A}_{x}^{N}:=\mathbb{A}^{N} \times_{\operatorname{Spec} k} \operatorname{Spec} k(x) \hookrightarrow \mathbb{A}^{2 N}, i_{x}: Z_{x}:=$ $Z \times \times_{\operatorname{Spec} k} \operatorname{Spec} k(x) \hookrightarrow \mathbb{A}_{x}^{N}$ be the closed immersions, and $\mathcal{E}_{x}:=\alpha_{x}^{!} \circ p_{1}^{!}(\mathcal{E})$ and $\rho_{x}: \operatorname{Spec} k(x) \rightarrow$ Spec $k$ the induced morphism. Since $p_{1} \circ \alpha_{x}$ is finite and étale, $\mathcal{H}_{\mathrm{t}}^{0} i_{x}^{!} \mathcal{E}_{x}=0$ and $\mathcal{E}_{x}$ is $\iota$-mixed of weight $\geq w$ outside $Z_{x}$. For a realizable variety $f: Y \rightarrow \operatorname{Spec}(k)$ and an overholonomic $F$-complex $\mathcal{F}$ over $Y$, we denote $\mathcal{H}_{\mathrm{t}}^{m}\left(f_{+}(\mathcal{F})\right)$ by $H^{m}(Y, \mathcal{F})$. By (1.1.9.1) and the base change, we get:

$$
\begin{aligned}
H^{m}\left(\mathbb{A}_{x}^{N}, \mathcal{E}_{x} \widetilde{\otimes} \alpha_{x}^{!} \mathcal{J}_{\pi}\right) & \stackrel{\sim}{\rightarrow} H^{m}\left(\mathbb{A}_{x}^{N}, \alpha_{x}^{!}\left(p_{1}^{!}(\mathcal{E}) \widetilde{\otimes} \mathcal{J}_{\pi}\right)\right) \\
& \stackrel{\sim}{\longrightarrow} \mathcal{H}_{\mathrm{t}}^{m}\left(\rho_{x,+} \circ \iota_{x}^{!} \circ p_{2+}\left(p_{1}^{!}(\mathcal{E}) \widetilde{\otimes} \mathcal{J}_{\pi}\right)\right) \cong \mathcal{H}_{\mathrm{t}}^{m}\left(\rho_{x,+} \circ \iota_{x}^{!}\left(T_{\pi}(\mathcal{E})\right)\right) .
\end{aligned}
$$

Since $T_{\pi}(\mathcal{E})[-N]$ is an $F$-module by 2.3.4.1, we get $H^{m}\left(\mathbb{A}_{x}^{N}, \mathcal{E}_{x} \widetilde{\otimes} \alpha_{x}^{!} \mathcal{J}_{\pi}\right)=0$ for any integer $m \notin[-N, 0]$ by (4.2.2.1). Moreover since $i_{x}^{!}\left(\mathcal{E}_{x} \widetilde{\otimes} \alpha_{x}^{!} \mathcal{J}_{\pi}\right) \stackrel{\sim}{\rightarrow} i_{x}^{!}\left(\mathcal{E}_{x}\right) \widetilde{\otimes} i_{x}^{!} \circ \alpha_{x}^{!} \mathcal{J}_{\pi}, i_{x}^{!} \circ \alpha_{x}^{!} \mathcal{J}_{\pi}$ is a $F$-module, and $\mathcal{H}_{\mathrm{t}}^{0} i_{x}^{!} \mathcal{E}_{x}=0$, we get that $H^{m}\left(Z_{x}, i_{x}^{!}\left(\mathcal{E}_{x} \widetilde{\otimes} \alpha_{x}^{!} \mathcal{J}_{\pi}\right)\right)=0$ for any integer $m \notin[1, N]$. Now, the exact sequence

$$
\rightarrow H^{m}\left(Z_{x}, i_{x}^{!}\left(\mathcal{E}_{x} \widetilde{\otimes} \alpha_{x}^{!} \mathcal{J}_{\pi}\right)\right) \rightarrow H^{m}\left(\mathbb{A}_{x}^{N}, \mathcal{E}_{x} \widetilde{\otimes} \alpha_{x}^{!} \mathcal{J}_{\pi}\right) \rightarrow H^{m}\left(\mathbb{A}_{x}^{N} \backslash Z_{x}, \mathcal{E}_{x} \widetilde{\otimes} \alpha_{x}^{!} \mathcal{J}_{\pi}\right) \rightarrow
$$

implies that

$$
H^{m}\left(\mathbb{A}_{x}^{N}, \mathcal{E}_{x} \widetilde{\otimes} \alpha_{x}^{!} \mathcal{J}_{\pi}\right) \begin{cases}\cong H^{m}\left(\mathbb{A}_{x}^{N} \backslash Z_{x}, \mathcal{E}_{x} \widetilde{\otimes} \alpha_{x}^{!} \mathcal{J}_{\pi}\right) & \text { for }-N \leq m<0 \\ \hookrightarrow H^{0}\left(\mathbb{A}_{x}^{N} \backslash Z_{x}, \mathcal{E}_{x} \widetilde{\otimes} \alpha_{x}^{!} \mathcal{J}_{\pi}\right) & \text { for } m=0 \\ =0 & \text { otherwise. }\end{cases}
$$


By Theorem 4.1.3, $H^{m}\left(\mathbb{A}_{x}^{N} \backslash Z_{x}, \mathcal{E}_{x} \widetilde{\otimes} \alpha_{x}^{!} \mathcal{J}_{\pi}\right)$ is $\iota$-mixed of weight $\geq w+m$ (recall that $\mathcal{J}_{\pi}$ is $\iota$-pure of weight 0 ), and thus from $(4.2 .2 .1), \iota_{x}^{!}\left(T_{\pi}(\mathcal{E})\right)$ is of weight $\geq w$. Hence, $T_{\pi}(\mathcal{E})$ is $\iota$-mixed of weight $\geq w$. By Theorem 4.1.3 and 2.3.4.2, we note that $\mathcal{F} \in D_{\geq w}^{\mathrm{b}}\left(\mathbb{A}^{N}\right)$ if and only if $T_{\pi}(\mathcal{F}) \in D_{\geq w}^{\mathrm{b}}\left(\mathbb{A}^{N}\right)$, and the theorem follows.

Theorem 4.2.3. Let $X$ be a realizable variety, $\mathcal{E} \in F$ - $D_{\mathrm{ovhol}}^{\mathrm{b}}(X / K)$, and $\star \in\{\emptyset, \leq w, \geq w\}$. If $\mathcal{E}$ is $\iota$-mixed of weight $\star$, then $\mathcal{E}$ satisfies the condition $(\mathrm{SQ})_{\star}$.

Proof. By Remark 2.2.3 (i) and duality, it is sufficient to consider the case where $\star$ is $\geq w$. We prove the theorem by induction on the dimension of $X$. The case where $\operatorname{dim}(X)=0$ is obvious. If we have a distinguished triangle $\mathcal{E}^{\prime} \rightarrow \mathcal{E} \rightarrow \mathcal{E}^{\prime \prime} \stackrel{+}{\rightarrow}$ in $F$ - $D_{\text {ovhol }}^{\mathrm{b}}(X / K)$ such that $(\mathrm{SQ})_{\geq w}$ holds for $\mathcal{E}^{\prime}$ and $\mathcal{E}^{\prime \prime}$, then $\mathcal{E}$ satisfies $(\mathrm{SQ})_{>w}$ as well. Hence, by dévissage, it suffices to show that for any smooth irreducible open subscheme $j: U \hookrightarrow X$ such that $j$ is affine and for any irreducible overconvergent $F$-isocrystal $\mathcal{E}$ on $U$ which is $\iota$-pure of weight $w$, the $F$-module $j_{+} \mathcal{E}$ satisfies $(\mathrm{SQ})_{>w}$. For this, let $\mathcal{F}$ be an overholonomic $F$-module on $X$ defined by the exact sequence $0 \rightarrow j_{!_{+}} \mathcal{E} \rightarrow j_{+} \mathcal{E} \rightarrow \mathcal{F} \rightarrow 0$. Let $Z:=X \backslash U$. By Theorem 4.1.3, the $F$-complex $\mathbb{R} \underline{\Gamma}_{Z}^{\dagger}\left(j_{!}(\mathcal{E})\right)$ is $\iota$-mixed. Since $\left.\mathbb{R} \underline{\Gamma}_{Z}^{\dagger}\left(j_{+}(\mathcal{E})\right)\right)=0$, one gets $\mathcal{F} \stackrel{\sim}{\rightarrow} \mathcal{H}_{\mathrm{t}}^{1}\left(\mathbb{R} \underline{\Gamma}_{Z}^{\dagger}\left(j_{!}(\mathcal{E})\right)\right)$, and the induction hypothesis shows that this satisfies the $(\mathrm{SQ})$ property and a fortiori is $\iota$-mixed. Hence $j_{!+} \mathcal{E}$ is $\iota$-mixed. From Theorem 4.2.2, By Corollary 1.4.3 and Proposition 1.4.7, we get that $j_{!_{+}} \mathcal{E}$ is irreducible and $\iota$-pure of weight $w$, and hence satisfies $(\mathrm{SQ})_{>w}$. Since $\mathcal{F} \stackrel{\sim}{\rightarrow} \mathcal{H}_{\mathrm{t}}^{1}\left(\mathbb{R} \underline{\Gamma}_{Z}^{\dagger}\left(j_{!_{+}}(\mathcal{E})\right)\right), \mathcal{F}$ is $\iota$-mixed of weight $\geq w+1$. Thus, by induction hypothesis, $\mathcal{F}$ satisfies $(\mathrm{SQ})_{\geq w+1}$ and a fortiori $(\mathrm{SQ})_{\geq w}$. Thus the theorem follows.

We see easily that the theorem leads to:

Corollary 4.2.4. Let $j: U \hookrightarrow X$ be an open immersion of realizable varieties. The intermediate extension $j !+$ sends an $\iota$-pure $F$-module on $U$ of weight $w$ to an $\iota$-pure $F$-module on $X$ of weight $w$.

\subsection{Applications}

This subsection is devoted to exhibit a few applications of the theory of weights. Let $*$ be either $\mathcal{D}_{X, \mathbb{Q}}^{\dagger}$ or $F-\mathcal{D}_{X, \mathbb{Q}}^{\dagger}$. We denote by $\mathrm{Hom}_{*}$ and $\mathrm{Ext}_{*}^{1}$ the Hom group and Yoneda's Ext group of the abelian category of overholonomic $*$-modules respectively.

Theorem 4.3.1 (Semi-simplicity of pure $F$-modules). Let $X$ be a realizable variety, and $\mathcal{E}$ be an ı-pure $F$-module in $F-O \operatorname{vhol}(X / K)$. Then $\mathcal{E}$ is semi-simple in $\operatorname{Ovhol}(X / K)$ (not in $F-O \operatorname{vhol}(X / K))$.

Proof. Let $\mathcal{F} \subset \mathcal{E}$ be the maximal semi-simple submodule in $\operatorname{Ovhol}(X / K)$. Then we see that $\mathcal{F}$ is stable under the Frobenius structure of $\mathcal{E}$, and the inclusion $\mathcal{F} \subset \mathcal{E}$ is in fact in $F$-Ovhol $(X / K)$. By Theorem 4.2.3, $\mathcal{F}$ and $\mathcal{E} / \mathcal{F}$ are $\iota$-pure as well. This gives us $[\mathcal{E}] \in \operatorname{Ext}_{F-\mathcal{D}_{X, \mathbb{Q}}^{\dagger}}^{1}(\mathcal{E} / \mathcal{F}, \mathcal{F})$. Now, by using Proposition A.5, Theorem 4.1.3, and Remark A.4, we have $\operatorname{Ext}_{\mathcal{D}_{X, Q}^{\dagger}}^{1}(\mathcal{E} / \mathcal{F}, \mathcal{F})^{F}=0$. Since the canonical homomorphism $\alpha: \operatorname{Ext}_{F-\mathcal{D}_{X, \mathbb{Q}}^{\dagger}}^{1}(\mathcal{E} / \mathcal{F}, \mathcal{F}) \rightarrow \operatorname{Ext}_{\mathcal{D}_{X, \mathbb{Q}}^{\dagger}}^{1}(\mathcal{E} / \mathcal{F}, \mathcal{F})$ factors through $\operatorname{Ext}_{\mathcal{D}_{X, \mathbb{Q}}^{\dagger}}^{1}(\mathcal{E} / \mathcal{F}, \mathcal{F})^{F} \rightarrow \operatorname{Ext}_{\mathcal{D}_{X, \mathbb{Q}}^{\dagger}}^{1}(\mathcal{E} / \mathcal{F}, \mathcal{F})$, we obtain $\alpha([\mathcal{E}])=0$, which concludes the proof.

Lemma 4.3.2. Let $X$ be a realizable variety, and $\mathcal{E}, \mathcal{F}$ be objects in $F-\mathrm{Ovhol}(X / K)$. Then, we have the following short exact sequence:

$$
0 \rightarrow \operatorname{Hom}_{\mathcal{D}_{X, \mathbb{Q}}^{\dagger}}(\mathcal{E}, \mathcal{F})_{F} \rightarrow \operatorname{Ext}_{F-\mathcal{D}_{X, \mathbb{Q}}^{\dagger}}^{1}(\mathcal{E}, \mathcal{F}) \rightarrow \operatorname{Ext}_{\mathcal{D}_{X, \mathbb{Q}}^{\dagger}}^{1}(\mathcal{E}, \mathcal{F})^{F} \rightarrow 0
$$

Proof. Let $0 \rightarrow \mathcal{F} \stackrel{\alpha}{\rightarrow} \mathcal{G} \stackrel{\beta}{\rightarrow} \mathcal{E} \rightarrow 0$ be an extension of $\mathcal{D}_{X, \mathbb{Q}^{\dagger}}^{\dagger}$ modules, and denote by $[\mathcal{G}] \in \operatorname{Ext}_{\mathcal{D}_{X, \mathbb{Q}}^{\dagger}}^{1}(\mathcal{E}, \mathcal{F})$ the class defined by the extension. Let $\phi_{\mathcal{F}}: \mathcal{F} \rightarrow \mathcal{F}$ be the Frobenius structure of $\mathcal{F}$, and the same for $\phi_{\mathcal{E}}$. Then the class $F^{*}[\mathcal{G}]$ can be written as

$$
0 \rightarrow \mathcal{F} \stackrel{\alpha \circ \phi_{\mathcal{F}}}{\longrightarrow} \mathcal{G} \stackrel{\phi_{\mathcal{E}}^{-1} \circ \beta}{\longrightarrow} \mathcal{E} \rightarrow 0
$$


The condition $F^{*}[\mathcal{G}]=[\mathcal{G}]$ means that there exists a dotted arrow making the diagram commutative:

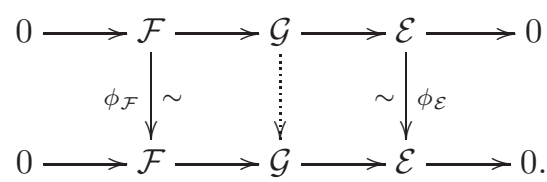

Thus the surjectivity of the homomorphism $\operatorname{Ext}_{F-\mathcal{D}_{X, \mathbb{Q}}^{\dagger}}^{1}(\mathcal{E}, \mathcal{F}) \rightarrow \operatorname{Ext}_{\mathcal{D}_{X, \mathbb{Q}}^{\dagger}}^{1}(\mathcal{E}, \mathcal{F})^{F}$ follows. Now, assume that the image of $\left[\left(\mathcal{G}, \phi_{\mathcal{G}}\right)\right]$ is 0 . This means that $\mathcal{G}$ is split. Then the computation of the kernel is standard. (See [BBD82, proof of 5.1.2] for example.)

Lemma 4.3.3. Let $X$ be a realizable variety, and $\mathcal{E}, \mathcal{F}$ be objects in $F-\mathrm{Ovhol}(X / K)$. Assume that $\mathcal{E}, \mathcal{F}$ are

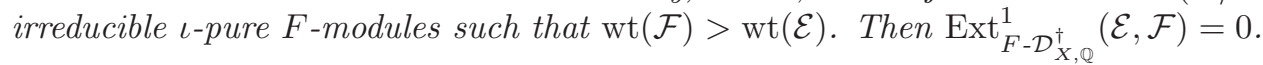

Proof. It suffices to show that the two outer modules appearing in the short exact sequence of Lemma 4.3.2 vanish. By Proposition A.5 and Theorem 4.1.3, we get the claim.

Theorem 4.3.4 (Weight filtration). Let $X$ be a realizable variety, and $\mathcal{E}$ be an object of $F$-Ovhol $(X / K)$ which is ı-mixed. Then there exists a unique increasing filtration $W$ on $\mathcal{E}$ such that $\operatorname{gr}_{i}^{W}(\mathcal{E})$ is purely of $\iota$-weight $i$. Any homomorphism of $\iota$-mixed $F$-modules on $X$ is strictly compatible with the filtration.

Proof. Using Lemma 4.3.3, we can follow the proof of [BBD82, 5.3.6].

4.3.5. Let $X$ be a realizable variety, and $\mathcal{E}, \mathcal{F} \in F$ - $D_{\text {ovhol }}^{\mathrm{b}}(X / K)$. By definition, we have the equality:

$$
\operatorname{Hom}_{F-D_{\text {ovhol }}^{\mathrm{b}}(X / K)}(\mathcal{E}, \mathcal{F})=\operatorname{Hom}_{D_{\text {ovhol }}^{\mathrm{b}}(X / K)}(\mathcal{E}, \mathcal{F})^{F} .
$$

By Theorem 4.1.3 and Proposition A.5, we get that if $\mathcal{E} \in F-D_{\leq w}^{\mathrm{b}}(X / K)$ and $\mathcal{F} \in F-D_{>w}^{\mathrm{b}}(X / K)$ then

$$
\operatorname{Hom}_{F-D_{\text {ovhol }}^{\mathrm{b}}(X / K)}(\mathcal{E}, \mathcal{F})=0 .
$$

Theorem 4.3.6 (Semi-simplicity). Let $X$ be a realizable variety, and $\mathcal{E}$ be an ı-pure overholonomic $F$ complex on $X$. Then $\mathcal{E}$ is isomorphic, in $D_{\text {ovhol }}^{\mathrm{b}}(X / K)$, to $\bigoplus_{n \in \mathbb{Z}} \mathcal{H}_{\mathrm{t}}^{n}(\mathcal{E})[n]$.

Proof. Using (4.3.5.1), the proof is essentially the same as that of [BBD82, 5.4.5, 5.4.6].

Remark 4.3.7. For a scheme $X$ over a field, denote by $D_{\mathrm{c}}^{\mathrm{b}}(X)$ the bounded derived category of constructible $\overline{\mathbb{Q}}_{\ell}$-complexes where $\ell$ is a prime number different from $p$. Let $X$ be a variety over the finite field $k$. As pointed out in Remark 1.1.5, the category $(F-) D_{\text {ovhol }}^{\mathrm{b}}(X / K)$ is an analogue of the category of Weil complexes $(F-) D_{\mathrm{c}}^{\mathrm{b}}\left(X \otimes_{k} \bar{k}\right)$, and should not be regarded as an analogue of the derived category $D_{c}^{\mathrm{b}}(X)$. In the $\ell$-adic setting [BBD82, 5.4.5], the corresponding theorem to Theorem 4.3.6 is stated for complexes in $D_{\mathrm{c}}^{\mathrm{b}}(X)$, so there are slight differences in the formulation arising from the lack of the category corresponding to $D_{\mathrm{c}}^{\mathrm{b}}(X)$ in our theory. However, since there exists the factorization

$$
D_{\mathrm{c}}^{\mathrm{b}}(X) \rightarrow F-D_{\mathrm{c}}^{\mathrm{b}}\left(X \otimes_{k} \bar{k}\right) \rightarrow D_{\mathrm{c}}^{\mathrm{b}}\left(X \otimes_{k} \bar{k}\right),
$$

if the true derived category corresponding to $D_{\mathrm{c}}^{\mathrm{b}}(X)$ in the $p$-adic cohomology theory is constructed, Theorem 4.3.6 should not lose any information.

4.3.8. We conclude this paper by showing an application to $\ell$-independence results of $L$-functions. To do this, we need to introduce, for a realizable variety $X$ over a finite field $k$, the category $F$ - $D_{\text {ovhol }}^{\mathrm{b}}\left(X / \overline{\mathbb{Q}}_{p}\right)$. The construction is essentially the same as [AM11, 7.3]. Let $L$ be a finite extension of the maximal unramified extension $K^{\mathrm{ur}}$. An object of $F$ - $D_{\text {ovhol }}^{\mathrm{b}}(X / L)$ is a pair $(\mathcal{E}, \rho)$ where $\mathcal{E} \in F$ - $D_{\text {ovhol }}^{\mathrm{b}}\left(X / K^{\mathrm{ur}}\right)$ and $\rho: L \rightarrow$ $\operatorname{End}_{F-D_{\text {ovhol }}^{\mathrm{b}}\left(X / K^{\mathrm{ur}}\right)}(\mathcal{E})$ is a homomorphism of $K^{\mathrm{ur}}$-algebras. By taking the cohomology functor $\mathcal{H}_{\mathrm{t}}^{*}, \rho$ induces a homomorphism $\mathcal{H}_{\mathrm{t}}^{*}(\rho): L \rightarrow \operatorname{End}_{F-\operatorname{Ovhol}\left(X / K^{\mathrm{ur}}\right)}\left(\mathcal{H}_{\mathrm{t}}^{*}(\mathcal{E})\right)$. The "heart" is denoted by $F$-Ovhol $(X / L)$. 
For finite extensions $L^{\prime} / L / K^{\mathrm{ur}}$, there exists a scalar extension functor $F$ - $D_{\mathrm{ovhol}}^{\mathrm{b}}(X / L) \rightarrow F-D_{\text {ovhol }}^{\mathrm{b}}\left(X / L^{\prime}\right)$. This is "t-exact" in the obvious sense, and induces a functor $F$-Ovhol $(X / L) \rightarrow F$-Ovhol $\left(X / L^{\prime}\right)$. By taking the limit over finite extensions $L$ of $K^{\text {ur }}$, we have the categories $F$ - $D_{\text {ovhol }}^{\mathrm{b}}\left(X / \overline{\mathbb{Q}}_{p}\right)$ and $F$-Ovhol $\left(X / \overline{\mathbb{Q}}_{p}\right)$. We notice that $F$-Ovhol $\left(\operatorname{Spec}(k) / \overline{\mathbb{Q}}_{p}\right)$ is nothing but the category of pairs $(V, \phi)$ where $V$ is a finite dimensional $\overline{\mathbb{Q}}_{p}$-vector space, and $\phi$ is an automorphism of $V$.

Since $\iota$-weights remain to be the same after scalar extension, Theorem 4.1.3, Theorem 4.2.3, Corollary 4.2.4 remain to be true even after replacing $K$ by $\overline{\mathbb{Q}}_{p}$.

Remark. Just to state Theorem 4.3.11, we do not need to introduce this generalized category. However, in the proof, we need to extend the scalar to $\overline{\mathbb{Q}}_{p}$, in order to produce sufficiently many sheaves $\mathcal{L}_{\rho}$ using the notation of [Fuj02, §3].

4.3.9. Let $X$ be a realizable variety over $k$. Let $\mathcal{E}$ be an object in $F$-Ovhol $\left(X / \overline{\mathbb{Q}}_{p}\right)$. For a closed point $x$ of $X$, let $\rho_{x}: \operatorname{Spec}(k(x)) \rightarrow \operatorname{Spec}(k)$ be the structural morphism. We define the local $L$-factor at $x$ to be

$$
L_{x}(\mathcal{E}, t):=\operatorname{det}_{\overline{\mathbb{Q}}_{p}}\left(1-t^{\operatorname{deg}(x)} \cdot F ; \rho_{x+} \circ i_{x}^{+}(\mathcal{E})\right)^{-1 / \operatorname{deg}(x)},
$$

and the global L-function ${ }^{(10)}$ to be

$$
L(X, \mathcal{E}, t):=\prod_{x \in|X|} L_{x}(\mathcal{E}, t)
$$

Let $K\left(X, \overline{\mathbb{Q}}_{p}\right)$ be the Grothendieck category of $F$-Ovhol $\left(X / \overline{\mathbb{Q}}_{p}\right)$. By additivity, the definition of local $L$-factor extends to a homomorphism

$$
K\left(X, \overline{\mathbb{Q}}_{p}\right) \rightarrow \prod_{x \in|X|}\left(1+t \cdot \overline{\mathbb{Q}}_{p} \llbracket t \rrbracket\right)^{\times} .
$$

Definition 4.3.10. Let $\ell$ be a prime number different from $p$, and $E$ be a field of characteristic 0 . We fix embeddings $\overline{\mathbb{Q}}_{p} \hookleftarrow E \hookrightarrow \overline{\mathbb{Q}}_{\ell}$, where the first one is denoted by $\iota_{p}$ and the second by $\iota_{\ell}$. A pair $(\mathcal{E}, \mathscr{F}) \in$ $K\left(X, \overline{\mathbb{Q}}_{p}\right) \times K\left(X, \overline{\mathbb{Q}}_{\ell}\right)$ is said to be an E-compatible system if for any closed point $x$ of $X$, we have

$$
\iota_{p}\left(L_{x}(\mathcal{E}, t)\right)=\iota_{\ell}\left(L_{x}(\mathscr{F}, t)\right) \in E \llbracket t \rrbracket .
$$

Theorem 4.3.11 (Fujiwara-Gabber's $\ell$-independence). Compatible systems are stable under $f_{+}, f^{!}, \mathbb{D}, \otimes$. Moreover, let $j: U \hookrightarrow X$ be an immersion of realizable varieties, and $(\mathcal{E}, \mathscr{F})$ be an $E$-compatible system such that $\mathcal{E}$ (and hence $\mathscr{F})$ is $\iota_{p}$-pure. Then $\left(j_{!_{+}}(\mathcal{E}), j_{! *}(\mathscr{F})\right)$ is also an E-compatible system.

Proof. The proof is exactly the same as [Fuj02]. In the proof, we need to use Lefschetz fixed point theorem. Since we are using "the extended scalar", we need to check the theorem in this situation. For the verification, it is reduced to the overconvergent $F$-isocrystal case by the same argument as [Car06]. We repeat the dévissage argument of [ÉLS93, 6.2] to reduce to the case where the fixed point is empty, and in this case we repeat [ÉLS93, 5.4]. We leave the detailed verifications to the reader.

4.3.12. Let $\mathcal{K}$ be a function field over $k$ (i.e. a field of transcendental degree 1 over $k$ ). We define

$$
F-\operatorname{Isoc}^{\dagger}(\mathcal{K} / K):=\underset{U}{\lim } F-\operatorname{Isoc}^{\dagger}(U / K)
$$

where the inductive limit runs over smooth curves over $k$ whose function field is $\mathcal{K}$.

Now, take $E$ in $F-\operatorname{Isoc}^{\dagger}(\mathcal{K} / K)$. By definition, there exists a smooth curve $U$ whose function field is $\mathcal{K}$, and $\widetilde{E}$ be an overconvergent $F$-isocrystal on $U$. Let $j: U \hookrightarrow X$ be the smooth compactification. We define the Hasse-Weil L-function by

$$
L_{\mathrm{HW}}(\mathcal{K}, E, t):=L\left(X, j_{!+}\left(\mathrm{sp}_{+}(\widetilde{E})\right), t\right) \in E \llbracket t \rrbracket .
$$

\footnotetext{
(10) There are several ways of normalizations of global $L$-function. Ours is the same as that of [AM11, 7.2.3].
} 
From 1.4.5, we check that this Hasse-Weil $L$-function does not depend on the choice of $U$ and of the smooth compactification $X$ of $U$. By Theorem 1.5.9 (iii), we may write the $L$-function as

$$
L_{\mathrm{HW}}(\mathcal{K}, E, t)=\prod_{x \in|X|} \operatorname{det}_{K}\left(1-\varphi_{x} \cdot t^{\operatorname{deg}(x)} ; \Psi_{x}(\widetilde{E})^{N=0, I=1}\right)^{-1 / \operatorname{deg}(x)},
$$

where we used the notation of paragraph 2.3.3. In particular, our Hasse-Weil $L$-function is nothing but $L_{\mathrm{WD}}(X, \widetilde{E}(1), t)$ in $[$ Mar08, 4.3.10] by Theorem 1.5.9 (i).

On the other hand, by Lefschetz trace formula and self-duality 1.4.3, we get the functional equation

$$
L_{\mathrm{HW}}(\mathcal{K}, E, t)=\varepsilon(\mathcal{K}, E) \cdot t^{g(\mathcal{K})-2} \cdot L_{\mathrm{HW}}\left(\mathcal{K}, E^{\vee}, q \cdot t^{-1}\right)
$$

where $g(\mathcal{K})$ denotes the genus of the function field $\mathcal{K}$, and $\varepsilon(\mathcal{K}, E)$ is some number in $\overline{\mathbb{Q}}_{p}$ (cf. [AM11, 7.2.3]). This is a generalization of [Mar08, 4.3.11] to $F$-isocrystals of arbitrary ranks.

Now, let $X$ be a realizable variety over $\mathcal{K}$. Then there exists a model $f: \mathcal{X} \rightarrow U$ where $U$ is a smooth curve whose function field is $\mathcal{K}, \mathcal{X}$ is a realizable variety, and the generic fiber of $f$ is $X$. We denote by $p: \mathcal{X} \rightarrow \operatorname{Spec}(k)$ the structural morphism. We define the cohomology $\mathcal{H}^{m}(X / K)$ in $F-\operatorname{Isoc}^{\dagger}(\mathcal{K} / K)$ to be the one represented by $\mathcal{H}_{\mathrm{t}}^{m}\left(f_{+} p^{+}(K)\right)$. We can check easily that the construction does not depend on auxiliary choices. We end this paper with the following $p$-adic interpretation of Hasse-Weil $L$-function over function fields, which follows directly from Theorem 4.3.11:

Corollary 4.3.13. Let $f: X \rightarrow \operatorname{Spec}(\mathcal{K})$ be a realizable proper smooth scheme (e.g. projective smooth variety). Then we have

$$
L_{\mathrm{HW}}\left(\mathcal{K}, H^{m}(\bar{X}), t\right)=L_{\mathrm{HW}}\left(\mathcal{K}, \mathcal{H}^{m}(X / K), t\right),
$$

where the left hand side is the Hasse-Weil L-function associated to the $\ell$-adic representation $H^{m}(\bar{X}):=$ $H^{m}\left(X \otimes \mathcal{K}^{\mathrm{sep}}, \mathbb{Q}_{\ell}\right)$ of $\operatorname{Gal}\left(\mathcal{K}^{\mathrm{sep}} / \mathcal{K}\right)$.

\section{A Internal homomorphism}

In this appendix, we consider situation (A) in Notation and convention.

Definition A.1. Let $\mathbb{Y}$ be a couple (1.1.2). Let $\mathcal{E}, \mathcal{F}, \mathcal{G} \in F-D_{\text {ovhol }}^{\mathrm{b}}(\mathbb{Y} / K)$. We put

$$
\mathcal{H} \mathrm{om}_{\mathbb{Y}}(\mathcal{E}, \mathcal{F}):=\mathbb{D}_{\mathbb{Y}}(\mathcal{E}) \widetilde{\otimes}_{\mathbb{Y}} \mathcal{F} \text {. }
$$

From the biduality isomorphism, we get the isomorphisms

$$
\mathcal{H o m}_{\mathbb{Y}}(\mathcal{E}, \mathcal{F}) \stackrel{\sim}{\rightarrow} \mathcal{H} \operatorname{com}_{\mathbb{Y}}\left(\mathbb{D}_{\mathbb{Y}}(\mathcal{F}), \mathbb{D}_{\mathbb{Y}}(\mathcal{E})\right), \quad \mathcal{H o m}_{\mathbb{Y}}\left(\mathcal{E}, \mathcal{H} \operatorname{om}_{\mathbb{Y}}(\mathcal{F}, \mathcal{G})\right) \stackrel{\sim}{\rightarrow} \mathcal{H o m} \mathrm{m}_{\mathbb{Y}}(\mathcal{E} \otimes \mathcal{F}, \mathcal{G}) .
$$

For any morphism $u: \mathbb{Y}^{\prime} \rightarrow \mathbb{Y}$ of couples, by the biduality isomorphism and (1.1.9.1), we have

$$
u^{!} \mathcal{H o m}_{\mathbb{Y}}(\mathcal{E}, \mathcal{F}) \stackrel{\sim}{\rightarrow} \mathcal{H o m}_{\mathbb{Y}^{\prime}}\left(u^{+}(\mathcal{E}), u^{!}(\mathcal{F})\right) .
$$

Lemma A.2. Let $f: P \rightarrow S$ be a smooth morphism of noetherian schemes such that $p$ is nilpotent in $\mathcal{O}_{S}$. For any $\mathcal{E}^{(m)} \in D_{\mathrm{coh}}^{\mathrm{b}}\left(\mathcal{D}_{P / S}^{(m)}\right)$ and $\mathcal{F}^{(m)} \in D^{\mathrm{b}}\left(\mathcal{D}_{P / S}^{(m)}\right)$, we have the canonical isomorphism:

$$
f_{+}^{(m)}\left(\mathbb{D}_{P / S}^{(m)}\left(\mathcal{E}^{(m)}\right) \otimes_{\mathcal{O}_{P}}^{\mathbb{L}} \mathcal{F}^{(m)}\right)\left[-d_{P}\right] \stackrel{\sim}{\rightarrow} \mathbb{R} f_{*}\left(\mathbb{R} \mathcal{H} o m_{\mathcal{D}_{P / S}^{(m)}}\left(\mathcal{E}^{(m)}, \mathcal{F}^{(m)}\right)\right) .
$$

Proof. This is the composition of the following isomorphisms:

$$
\begin{aligned}
& f_{+}^{(m)}\left(\mathbb{D}_{P / S}^{(m)}\left(\mathcal{E}^{(m)}\right) \otimes_{\mathcal{O}_{P}}^{\mathbb{L}} \mathcal{F}^{(m)}\right)\left[-d_{P}\right] \underset{\star}{\stackrel{\sim}{\rightarrow}} f_{+}^{(m)}\left(\mathbb{R} \mathcal{H o m}_{\mathcal{D}_{P / S}^{(m)}}\left(\mathcal{E}^{(m)},\left(\mathcal{D}_{P / S}^{(m)} \otimes_{\mathcal{O}_{P}} \omega_{P / S}^{-1}\right) \otimes_{\mathcal{O}_{P}}^{\mathbb{L}} \mathcal{F}^{(m)}\right)\left[-d_{P}\right]\right) \\
& =\mathbb{R} f_{*}\left(\omega_{P / S} \otimes_{\mathcal{D}_{P / S}^{(m)}}^{\mathbb{L}} \mathbb{R} \mathcal{H} o m_{\mathcal{D}_{P / S}^{(m)}}\left(\mathcal{E}^{(m)},\left(\mathcal{D}_{P / S}^{(m)} \otimes_{\mathcal{O}_{P}} \omega_{P / S}^{-1}\right) \otimes_{\mathcal{O}_{P}}^{\mathbb{L}} \mathcal{F}^{(m)}\right)\right) \\
& \underset{\star \star}{\stackrel{\sim}{\longrightarrow}} \mathbb{R} f_{*}\left(\mathbb{R} \mathcal{H o m}{\mathcal{D}_{P / S}^{(m)}}\left(\mathcal{E}^{(m)}, \omega_{P / S} \otimes_{\mathcal{D}_{P / S}^{(m)}}^{\mathbb{L}}\left(\mathcal{D}_{P / S}^{(m)} \otimes_{\mathcal{O}_{P}} \omega_{P / S}^{-1}\right) \otimes_{\mathcal{O}_{P}}^{\mathbb{L}} \mathcal{F}^{(m)}\right)\right) \\
& \stackrel{\sim}{\rightarrow} \mathbb{R} f_{*}\left(\mathbb{R} \mathcal{H o m}{ }_{\mathcal{D}_{P / S}^{(m)}}\left(\mathcal{E}^{(m)}, \mathcal{F}^{(m)}\right)\right) .
\end{aligned}
$$

where $\star$ follows by [Car05, 2.1.26], and $\star \star$ follows by [Car05, 2.1.19]. 
Definition A.3. Let $\mathbb{Y}$ be a couple. Choose an l.p. frame $(Y, X, \mathcal{P}, \mathcal{Q})$ of $\mathbb{Y}$. Let $\mathcal{E}, \mathcal{F} \in D_{\text {ovhol }}^{\mathrm{b}}(\mathbb{Y} / K)$ and $\mathcal{E}_{\mathcal{P}}, \mathcal{F}_{\mathcal{P}}$ be the corresponding objects in $D_{\text {ovhol }}^{\mathrm{b}}(Y, \mathcal{P} / K)$. Then, using Lemma 1.2.8, we notice that the complex of abelian groups $\mathbb{R H o m}_{D\left(\mathcal{D}_{\mathcal{P}, \mathbb{Q}}^{\dagger}\right)}(\mathcal{E}, \mathcal{F})$ does not depend on the choice of the l.p. frame $(Y, X, \mathcal{P}, \mathcal{Q})$ of $\mathbb{Y}$. We put

$$
\mathbb{R H o m}_{D_{\text {ovhol }}^{\mathrm{b}}(\mathbb{Y} / K)}(\mathcal{E}, \mathcal{F}):=\mathbb{R H o m}_{D\left(\mathcal{D}_{\mathcal{P}, \mathbb{Q}}^{\dagger}\right)}\left(\mathcal{E}_{\mathcal{P}}, \mathcal{F}_{\mathcal{P}}\right) .
$$

Remark A.4. Let $\mathcal{E}, \mathcal{F}$ be objects of $\operatorname{Ovhol}(\mathbb{Y} / K)$. Then we can check easily that

$$
\mathcal{H}^{1} \mathbb{R} \operatorname{Hom}_{D_{\text {ovhol }}^{\mathrm{b}}(\mathbb{Y} / K)}(\mathcal{E}, \mathcal{F})=\operatorname{Ext}_{\mathcal{D}_{\mathbb{Y} / K}^{\dagger}}^{1}(\mathcal{E}, \mathcal{F}),
$$

where $\operatorname{Ext}_{\mathcal{D}_{\mathbb{Y} / K}^{\dagger}}^{1}(\mathcal{E}, \mathcal{F})$ denotes the Yoneda's Ext group in the abelian category $\operatorname{Ovhol}(\mathbb{Y} / K)$.

Proposition A.5. Let $\mathbb{Y}$ be a couple and $a: \mathbb{Y} \rightarrow \operatorname{Spec}(k)$ the structural morphism which we assume to be complete. For any $\mathcal{E}, \mathcal{F} \in F-D_{\text {ovhol }}^{\mathrm{b}}(\mathbb{Y} / K)$, we have the isomorphism

$$
a_{+} \mathcal{H o m}_{\mathbb{Y}}(\mathcal{E}, \mathcal{F}) \stackrel{\sim}{\rightarrow} \mathbb{R H o m}_{D_{\text {ovhol }}^{\mathrm{b}}(\mathbb{Y} / K)}(\mathcal{E}, \mathcal{F}) .
$$

Proof. Choose an l.p. frame $(Y, X, \mathcal{P}, \mathcal{Q})$ of $\mathbb{Y}$. Let $\mathcal{E}, \mathcal{F} \in F$ - $D_{\text {ovhol }}^{\mathrm{b}}(Y, \mathcal{P} / K)$. Let $f$ be the structural morphism of $\mathcal{P}$ and $Z:=X \backslash Y$. By definition, we get:

$$
\begin{aligned}
a_{+} \mathcal{H} \operatorname{om}_{\mathbb{Y}}(\mathcal{E}, \mathcal{F}) \cong f_{+}\left(\left({ }^{\dagger} Z\right) \circ \mathbb{D}_{\mathcal{P}}(\mathcal{E}) \stackrel{\mathbb{\otimes}}{\otimes}_{\mathcal{O}_{\mathcal{P}, \mathbb{Q}}} \mathcal{F}\right)\left[-d_{P}\right] \stackrel{\sim}{\rightarrow} f_{+}\left(\mathbb{D}_{\mathcal{P}}(\mathcal{E}) \stackrel{\mathbb{L}^{\dagger}}{\mathcal{O}_{\mathcal{P}, \mathbb{Q}}} \mathcal{F}\right)\left[-d_{P}\right], \\
\\
\mathbb{R} \operatorname{Hom}_{D_{\text {ovhol }}^{\mathrm{b}}(\mathbb{Y} / K)}(\mathcal{E}, \mathcal{F}) \cong \mathbb{R} \operatorname{Hom}_{D\left(\mathcal{D}_{\mathcal{P}, \mathbb{Q}}^{\dagger}\right)}(\mathcal{E}, \mathcal{F}) .
\end{aligned}
$$

Thus, we are reduced to the case where $Y=X=P$. Let $\mathcal{E}^{(\bullet)}, \mathcal{F}^{(\bullet)} \in \underline{L D_{\mathbb{Q}, \text { coh }}^{\mathrm{b}}}\left(\widehat{\mathcal{D}}_{\mathcal{P}}^{(\bullet)}\right)$ such that $\lim _{\longrightarrow} \mathcal{E}^{(\bullet)} \stackrel{\sim}{\rightarrow} \mathcal{E}$ and $\lim _{\longrightarrow} \mathcal{F}^{(\bullet)} \stackrel{\sim}{\rightarrow} \mathcal{F}$. We can suppose that there exists an increasing map $\lambda: \mathbb{N} \rightarrow \mathbb{N}$ such that for any integers $m^{\prime} \geq m$ we have $\lambda(m) \geq m, \mathcal{E}^{(m)} \in D_{\text {coh }}^{\mathrm{b}}\left(\widehat{\mathcal{D}}_{\mathcal{P}}^{(\lambda(m))}\right)$ and the canonical morphism

$$
\widehat{\mathcal{D}}_{\mathcal{P}}^{\left(\lambda\left(m^{\prime}\right)\right)} \otimes_{\widehat{\mathcal{D}}_{\mathcal{P}}^{(\lambda(m))}}^{\mathbb{L}} \mathcal{E}^{(m)} \rightarrow \mathcal{E}^{\left(m^{\prime}\right)}
$$

is an isomorphism. By taking projective limits, we deduce from Lemma A.2 the isomorphism

$$
f_{+}^{(\lambda(m))}\left(\mathbb{D}^{\lambda(m)}\left(\mathcal{E}^{(m)}\right) \widehat{\otimes}_{\mathcal{O}_{\mathcal{P}}}^{\mathbb{L}} \mathcal{F}^{(\lambda(m))}\right)\left[-d_{P}\right] \stackrel{\sim}{\rightarrow} \mathbb{R H o m}_{D\left(\widehat{\mathcal{D}}_{\mathcal{P}}^{(\lambda(m))}\right)}\left(\mathcal{E}^{(m)}, \mathcal{F}^{(\lambda(m))}\right) .
$$

Since $\mathcal{E}^{(m)} \in D_{\text {coh }}^{\mathrm{b}}\left(\widehat{\mathcal{D}}_{\mathcal{P}}^{(\lambda(m))}\right)$, we get the isomorphisms $\widehat{\mathcal{D}}_{\mathcal{P}}^{\left(\lambda\left(m^{\prime}\right)\right)} \otimes_{\widehat{\mathcal{D}}_{\mathcal{P}}^{(\lambda(m))}}^{\mathbb{L}} \mathbb{D}^{\lambda(m)}\left(\mathcal{E}^{(m)}\right) \rightarrow \mathbb{D}^{\lambda\left(m^{\prime}\right)}\left(\mathcal{E}^{\left(m^{\prime}\right)}\right)$ and $\widehat{\mathcal{D}}_{\mathcal{P}}^{\left(\lambda\left(m^{\prime}\right)\right)} \otimes_{\widehat{\mathcal{D}}_{\mathcal{P}}^{(\lambda(m))}}^{\mathbb{L}} \mathbb{R} \operatorname{Hom}_{D\left(\widehat{\mathcal{D}}_{\mathcal{P}}^{(\lambda(m))}\right)}\left(\mathcal{E}^{(m)}, \mathcal{F}^{\left(\lambda\left(m^{\prime}\right)\right)}\right) \stackrel{\sim}{\rightarrow} \mathbb{R H o m}_{D\left(\widehat{\mathcal{D}}_{\mathcal{P}}^{\left(\lambda\left(m^{\prime}\right)\right)}\right)}\left(\mathcal{E}^{\left(m^{\prime}\right)}, \mathcal{F}^{\left(\lambda\left(m^{\prime}\right)\right)}\right)$. By taking the direct limit to $(\star)$ and tensoring by $\mathbb{Q}$, we get the desired isomorphism.

Proposition A.6 (Projection formulas). Let $u: \mathbb{Y} \rightarrow \mathbb{Y}^{\prime}$ a complete morphism of couples. For any $\mathcal{E} \in$ $F-D_{\text {ovhol }}^{\mathrm{b}}(\mathbb{Y} / K), \mathcal{E}^{\prime} \in F-D_{\text {ovhol }}^{\mathrm{b}}\left(\mathbb{Y}^{\prime} / K\right)$,

$$
u_{+}\left(\mathcal{E} \widetilde{\otimes} u^{!}\left(\mathcal{E}^{\prime}\right)\right) \stackrel{\sim}{\longrightarrow} u_{+}(\mathcal{E}) \widetilde{\otimes} \mathcal{E}^{\prime}, \quad u_{!}\left(\mathcal{E} \otimes u^{+}\left(\mathcal{E}^{\prime}\right)\right) \stackrel{\sim}{\longrightarrow} u_{!}(\mathcal{E}) \otimes \mathcal{E}^{\prime}
$$

Proof. Let $u=(\star, \star, g, \star):(Y, X, \mathcal{P}, \mathcal{Q}) \rightarrow\left(Y^{\prime}, X^{\prime}, \mathcal{P}^{\prime}, \mathcal{Q}^{\prime}\right)$ be a morphism of frames for $u$. We put $Z:=X \backslash Y$ and $Z^{\prime}:=X^{\prime} \backslash Y^{\prime}$. Let $\mathcal{E} \in F-D_{\text {ovhol }}^{\mathrm{b}}(Y, \mathcal{P} / K)$ and $\mathcal{E}^{\prime} \in F-D_{\text {ovhol }}^{\mathrm{b}}\left(Y^{\prime}, \mathcal{P}^{\prime} / K\right)$. Then we have

$$
u_{+}\left(\mathcal{E} \widetilde{\otimes} u^{!}\left(\mathcal{E}^{\prime}\right)\right) \stackrel{\sim}{\rightarrow} g_{+}\left(\mathcal{E}^{\mathbb{L}} \otimes_{\mathcal{O}_{\mathcal{P}, Q}}^{\dagger} g^{!}\left(\mathcal{E}^{\prime}\right)\right)\left[-d_{P}\right], \quad u_{+}(\mathcal{E}) \widetilde{\otimes} \mathcal{E}^{\prime} \stackrel{\sim}{\rightarrow} g_{+}\left(\mathcal{E}_{\otimes_{\mathcal{O}_{\mathcal{P}^{\prime}, \mathbb{Q}}}^{\mathbb{L}}}^{\dagger} \mathcal{E}^{\prime}\right)\left[-d_{P^{\prime}}\right] .
$$

Hence, from the projection formula [Car04, 2.1.4], we get the first one. By using the biduality isomorphism, this implies the second one. 
Proposition A.7 (Adjointness Properties). Let $u: \mathbb{Y} \rightarrow \mathbb{Y}^{\prime}$ a morphism of couples. For any $\mathcal{E} \in F-D_{\text {ovhol }}^{\mathrm{b}}(\mathbb{Y} / K)$, $\mathcal{E}^{\prime} \in F-D_{\text {ovhol }}^{\mathrm{b}}\left(\mathbb{Y}^{\prime} / K\right)$, we get the functorial isomorphism:

$$
u_{+} \mathcal{H} \mathrm{om}_{\mathbb{Y}}\left(\mathcal{E}, u^{!}\left(\mathcal{E}^{\prime}\right)\right) \stackrel{\sim}{\rightarrow} \mathcal{H} \mathrm{om}_{\mathbb{Y}^{\prime}}\left(u_{!}(\mathcal{E}), \mathcal{E}^{\prime}\right) .
$$

Proof. We have

$$
u_{+} \mathcal{H} \operatorname{om}_{\mathbb{Y}}\left(\mathcal{E}, u^{!}\left(\mathcal{E}^{\prime}\right)\right)=u_{+}\left(\mathbb{D}_{\mathbb{Y}}(\mathcal{E}) \widetilde{\otimes} u^{!}\left(\mathcal{E}^{\prime}\right)\right) \underset{\star}{\stackrel{\sim}{\longrightarrow}}\left(u_{+} \circ \mathbb{D}_{\mathbb{Y}}\right)(\mathcal{E}) \widetilde{\otimes} \mathcal{E}^{\prime} \stackrel{\sim}{\rightarrow} \mathcal{H} \mathrm{om}_{\mathbb{Y}^{\prime}}\left(u_{!}(\mathcal{E}), \mathcal{E}^{\prime}\right) .
$$

where $\star$ follows by (A.6.1).

Proposition A.8. Let $u: \mathbb{Y} \rightarrow \mathbb{Y}^{\prime}$ a morphism of couples. For any $\mathcal{E} \in F$ - $D_{\text {ovhol }}^{\mathrm{b}}(\mathbb{Y} / K), \mathcal{E}^{\prime} \in F-D_{\text {ovhol }}^{\mathrm{b}}\left(\mathbb{Y}^{\prime} / K\right)$, we get the functorial isomorphism:

$$
u_{+} \mathcal{H o m}_{\mathbb{Y}}\left(u^{+}\left(\mathcal{E}^{\prime}\right), \mathcal{E}\right) \stackrel{\sim}{\rightarrow} \mathcal{H}_{\mathrm{om}_{\mathbb{Y}}^{\prime}}\left(\mathcal{E}^{\prime}, u_{+}(\mathcal{E})\right) .
$$

Proof. This is a consequence of (A.1.1) and Proposition A.7.

\section{REFERENCES}

[AM11] T. AbE et A. Marmora - "Product formula for $p$-adic epsilon factors", J. Inst. Math. Jussieu (2014).

[Abe11] T. ABE - "Langlands program for p-adic coefficients and the petits camarades conjecture", (2011).

[Abe13] T. ABE - "Explicit calculation of Frobenius isomorphisms and Poincaré duality in the theory of arithmetic $\mathcal{D}$ module", Rend. Sem. Mat. Univ. Padova (2013).

[BBD82] A. A. Beॉ́linson, J. Bernstein et P. Deligne - "Faisceaux pervers", Analysis and topology on singular spaces, I (Luminy, 1981), Astérisque, vol. 100, Soc. Math. France, Paris, 1982, p. 5-171.

[Ber84] P. Berthelot - "Cohomologie rigide et théorie de Dwork: le cas des sommes exponentielles", Astérisque (1984), no. 119-120, p. 3, 17-49, p-adic cohomology.

[Ber96b] - " "--modules arithmétiques. I. Opérateurs différentiels de niveau fini", Ann. Sci. École Norm. Sup. (4) 29 (1996), no. 2, p. 185-272.

[Ber00] - " "D-modules arithmétiques. II. Descente par Frobenius", Mém. Soc. Math. Fr. (N.S.) (2000), no. 81, p. vi+136.

[Ber02] _ , "Introduction à la théorie arithmétique des D-modules", Astérisque (2002), no. 279, p. 1-80, Cohomologies $p$-adiques et applications arithmétiques, II.

$\left[\right.$ BGK $\left.^{+} 87\right]$ A. Borel, P.-P. Grivel, B. Kaup, A. Haefliger, B. Malgrange et F. Ehlers - Algebraic D-modules, Academic Press Inc., Boston, MA, 1987.

[Car04] D. CARO - "D-modules arithmétiques surcohérents. Application aux fonctions L", Ann. Inst. Fourier, Grenoble 54 (2004), no. 6, p. 1943-1996.

[Car05] - "Comparaison des foncteurs duaux des isocristaux surconvergents", Rend. Sem. Mat. Univ. Padova 114 (2005), p. 131-211.

[Car06] - , "Fonctions L associées aux D-modules arithmétiques. Cas des courbes", Compositio Mathematica 142 (2006), no. 01, p. 169-206.

[Car06] - , "Dévissages des $F$-complexes de $\mathcal{D}$-modules arithmétiques en $F$-isocristaux surconvergents", Invent. Math. 166 (2006), no. 2, p. 397-456.

[Car07] - , "Overconvergent F-isocrystals and differential overcoherence", Invent. Math. 170 (2007), no. 3, p. 507-539.

[Car08] — , "Sur la stabilité par produits tensoriels des F-complexes de $\mathcal{D}$-modules arithmétiques", (2008).

[Car09a] - , "Log-isocristaux surconvergents et holonomie.", Compos. Math. 145 (2009), no. 6, p. 1465-1503.

[Car09b] - , "D-modules arithmétiques surholonomes", Ann. Sci. École Norm. Sup. (4) 42 (2009), no. 1, p. 141-192.

[Car09c] - , "D-modules arithmétiques associés aux isocristaux surconvergents. Cas lisse", Bull. Soc. Math. Fr., 137(4):453-543, 2009.

[Car11a] — , "Pleine fidélité sans structure de Frobenius et isocristaux partiellement surconvergents", Math. Ann. 349 (2011), p. $747-805$.

[Car11b] - , "Sur la préservation de la surconvergence par l'image directe d'un morphisme propre et lisse", to appear to Ann. Sci. École Norm. Sup.

[Car12a] — , "The formalism of Grothendieck's six operations in p-adic cohomologies", (2012).

[Car12b] _ , "Sur la préservation de la cohérence par image inverse extraordinaire par une immersion fermée", ArXiv Mathematics e-prints (2012). 
[Car12c] - "Systèmes inductifs surcohérents de D-modules arithmétiques", ArXiv Mathematics e-prints (2012).

[CT12] D. CARo et N. Tsuzuki - "Overholonomicity of overconvergent F-isocrystals over smooth varieties", Annals of Math. (2012).

[CM01] G. Christol et Z. Mebkhout - "Sur le théorème de l'indice des équations différentielles p-adiques. IV", Invent. Math. 143 (2001), no. 3, p. 629-672.

[Cre98] R. CREW - "Finiteness theorems for the cohomology of an overconvergent isocrystal on a curve", Ann. Sci. École Norm. Sup. (4) 31 (1998), no. 6, p. 717-763.

[Cre06] - "Arithmetic D-modules on a formal curve", Math. Ann. 336 (2006), no. 2, p. 439-448.

[Cre12] - "Arithmetic D-modules on the unit disk", Compos. Math. 148 (2012), no. 1, p. 227-268.

[Del80] P. Deligne - "La conjecture de Weil. II", Inst. Hautes Études Sci. Publ. Math. (1980), no. 52, p. 137-252.

[ÉLS93] J.-Y. ÉtesSE et B. Le Stum - "Fonctions $L$ associées aux $F$-isocristaux surconvergents. I. Interprétation cohomologique", Math. Ann. 296 (1993), no. 3, p. 557-576.

[Fuj02] K. Fujiwara - "Independence of $l$ for intersection cohomology (after Gabber)", Algebraic geometry 2000, Azumino (Hotaka), Adv. Stud. Pure Math., vol. 36, Math. Soc. Japan, Tokyo, 2002, p. 145-151.

[Har66] R. HARtshorne - Residues and duality, Springer-Verlag, Berlin, 1966.

[Kat89] Kazuya Kato. Logarithmic structures of Fontaine-Illusie. In Algebraic analysis, geometry, and number theory (Baltimore, MD, 1988), pages 191-224. Johns Hopkins Univ. Press, Baltimore, MD, 1989.

[KM74] N. M. KATZ et W. MEssing - "Some consequences of the Riemann hypothesis for varieties over finite fields", Invent. Math. 23 (1974), p. 73-77.

[Ked04] Kiran S. Kedlaya. Full faithfulness for overconvergent F-isocrystals. In Geometric aspects of Dwork theory. Vol. I, II, pages 819-835. Walter de Gruyter GmbH \& Co. KG, Berlin, 2004.

[Ked06] K. S. KedlayA - "Fourier transforms and p-adic 'Weil II"', Compos. Math. 142 (2006), no. 6, p. 1426-1450.

[Ked07] - , "Semistable reduction for overconvergent $F$-isocrystals. I. Unipotence and logarithmic extensions", Compos. Math. 143 (2007), no. 5, p. 1164-1212.

[Ked11] _ , "Semistable reduction for overconvergent $F$-isocrystals, IV: local semistable reduction at nonmonomial valuations", Compos. Math. 147 (2011), no. 2, p. 467-523.

[KW01] R. Kienl et R. Weissauer - Weil conjectures, perverse sheaves and l'adic Fourier transform, Ergebnisse der Mathematik und ihrer Grenzgebiete. 3. Folge. A Series of Modern Surveys in Mathematics [Results in Mathematics and Related Areas. 3rd Series. A Series of Modern Surveys in Mathematics], vol. 42, Springer-Verlag, Berlin, 2001.

[Lau87] G. LAumon - "Transformation de Fourier, constantes d'équations fonctionnelles et conjecture de Weil", Inst. Hautes Études Sci. Publ. Math. (1987), no. 65, p. 131-210.

[Mar08] A. Marmora - "Facteurs epsilon p-adiques", Compos. Math. 144 (2008), no. 2, p. 439-483.

[Mat02] Shigeki Matsuda. Katz correspondence for quasi-unipotent overconvergent isocrystals. Compositio Math., 134(1):1-34, 2002.

[Meb89] Z. Mebkhout. Le formalisme des six opérations de Grothendieck pour les $\mathcal{D}_{X}$-modules cohérents. Hermann, Paris, 1989. With supplementary material by the author and L. Narváez Macarro.

[Mon02] Claude Montagnon. Généralisation de la théorie arithmétique des $\mathcal{D}$-modules à la géométrie logarithmique. $\mathrm{PhD}$ thesis, Université de Rennes I, 2002.

[NH97] C. Noot-Huyghe - "D'†-affinité de l'espace projectif", Compositio Math. 108 (1997), no. 3, p. 277-318, With an appendix by P. Berthelot.

[NH04] _ , "Transformation de Fourier des D-modules arithmétiques. I", Geometric aspects of Dwork theory. Vol. I, II, Walter de Gruyter GmbH \& Co. KG, Berlin, 2004, p. 857-907.

[Ogu Arthur Ogus. Log book.

[Shi10] Atsushi Shiho. On logarithmic extension of overconvergent isocrystals. Math. Ann., 348(2):467-512, 2010.

[Tsu02] Nobuo Tsuzuki. Morphisms of $F$-isocrystals and the finite monodromy theorem for unit-root $F$-isocrystals. $D u k e$ Math. J., 111(3):385-418, 2002.

[Vir00] A. Virrion - "Dualité locale et holonomie pour les D-modules arithmétiques", Bull. Soc. Math. France 128 (2000), no. 1, p. 1-68.

[Vir04] _ , "Trace et dualité relative pour les D-modules arithmétiques", Geometric aspects of Dwork theory. Vol. I, II, Walter de Gruyter GmbH \& Co. KG, Berlin, 2004, p. 1039-1112.

Tomoyuki Abe:

Kavli Institute for the Physics and Mathematics of the Universe

The University of Tokyo

5-1-5 Kashiwanoha, Kashiwa, Chiba, 277-8583, Japan

e-mail: tomoyuki.abe@ipmu.jp 
Daniel Caro:

Laboratoire de Mathématiques Nicolas Oresme (LMNO)

Université de Caen, Campus 2

14032 Caen Cedex, France

e-mail: daniel.caro@unicaen.fr 